\title{
Real-world treatment outcomes in metastatic lung cancer
}

\author{
From efficacy-effectiveness gap evaluation \\ to informed clinical decision-making
}

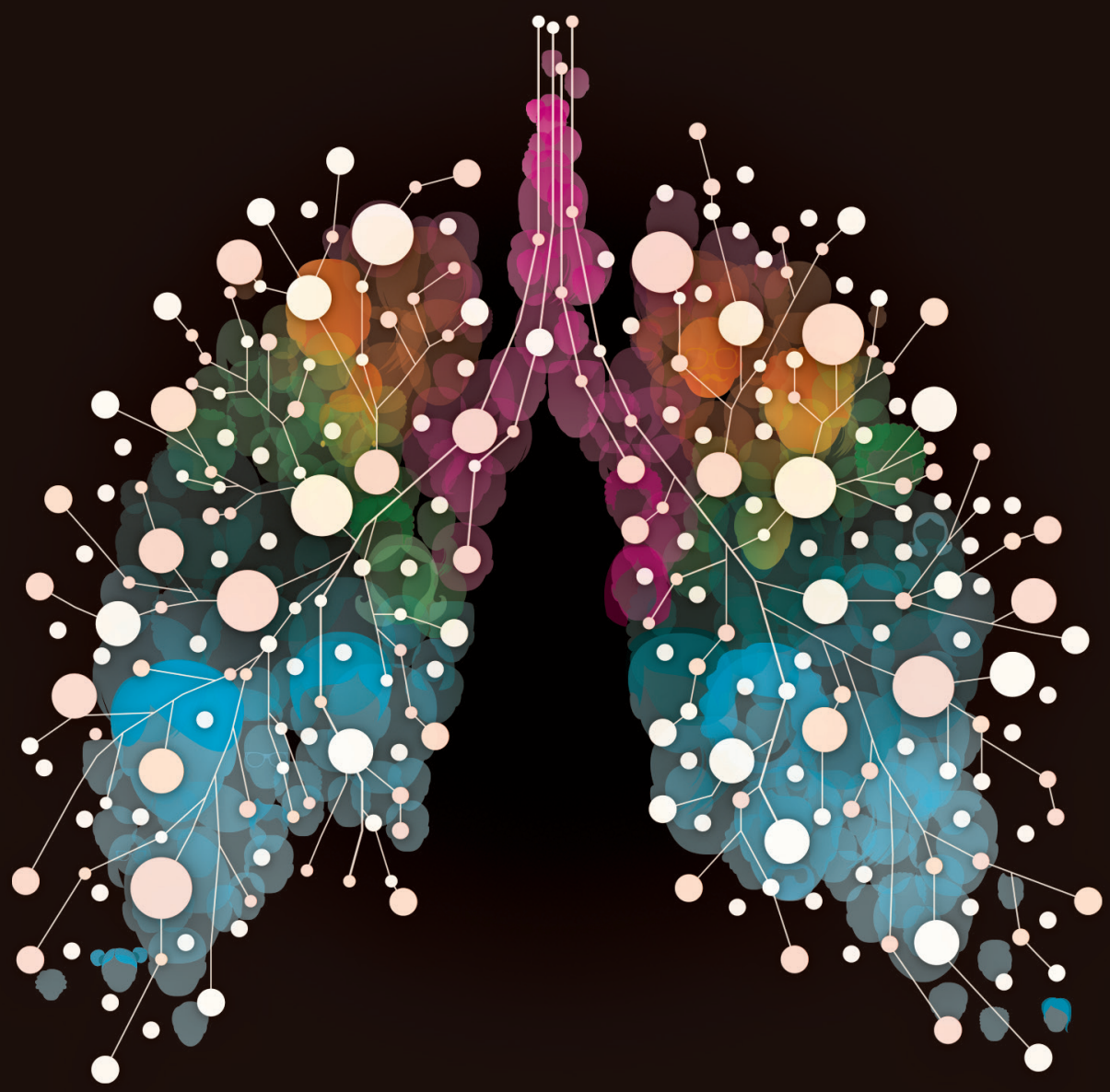

C.M. Cramer - van der Welle 



\section{Real-world treatment outcomes in metastatic lung cancer}

From efficacy-effectiveness gap evaluation to informed clinical decision-making 


\section{ISBN}

978-94-6421-262-4

\section{Design/lay-out}

Promotie In Zicht (www.promotie-inzicht.nl)

\section{Print}

Ipskamp Printing

c) C.M. Cramer - van der Welle, 2021

All rights are reserved. No part of this book may be reproduced, distributed, stored in a retrieval system, or transmitted in any form or by any means, without prior written permission of the author. 


\section{Real-world treatment outcomes in metastatic lung cancer}

From efficacy-effectiveness gap evaluation to informed clinical decision-making

Behandeluitkomsten in de dagelijkse praktijk

bij uitgezaaide longkanker

Van vergelijken met klinische studies

naar geïnformeerd kiezen

(met een samenvatting in het Nederlands)

\section{Proefschrift}

ter verkrijging van de graad van doctor aan de

Universiteit Utrecht

op gezag van de rector magnificus,

prof.dr. H.R.B.M. Kummeling,

ingevolge het besluit van het college voor promoties

in het openbaar te verdedigen op

maandag 29 maart 2021 des middags te 2.30 uur

door

Christina Maaike van der Welle

geboren op 24 mei 1985

te Apeldoorn 


\section{Promotoren}

Prof. dr. O.H. Klungel

Prof. dr. H.J.M. Groen

\section{Copromotoren}

Dr. E.M.W. van de Garde

Dr. F.M.N.H. Schramel

Dit proefschrift werd mede mogelijk gemaakt met financiële steun van KWF Kankerbestrijding. 


\section{CONTENTS}

$\begin{array}{ll}\text { General introduction } & 7\end{array}$

Part I Real-world outcomes NSCLC

Chapter 1 'Care for Outcomes': systematic development of a set of 19 outcome indicators to improve patient-relevant outcomes for patients with lung cancer BMJ Open. 2021 Jan 15;11(1):e043229

Chapter 2 Trends in prescribing systemic treatment and overall survival for non-small cell lung cancer stage IIIB/IV in the Netherlands: 2008-2012 Cancer Epidemiol. 2017 Dec;51:1-6

Chapter 3 Systematic evaluation of the efficacy-effectiveness gap of systemic treatments in metastatic non-small cell lung cancer Eur Respir J. 2018 Dec 20;52(6):1801100

Chapter 4 Trends in drug costs and overall survival in patients with metastatic non-small cell lung cancer in the Netherlands diagnosed from 2008 through 2014 Pharmacoecon Open. 2021 Mar;5(1):121-127.

Chapter 5 Real-world outcomes versus clinical trial results of immunotherapy in stage IV non-small cell lung cancer (NSCLC) in the Netherlands Accepted for publication in Scientific Reports

Part II Real-world outcomes SCLC

Chapter 6 Real-world treatment patterns and outcomes of patients with extensive disease small cell lung cancer Eur J Cancer Care (Engl.) 2020 Sep;29(5):e13250

Chapter 7 Systematic evaluation of the efficacy-effectiveness gap of systemic treatments in extensive disease small cell lung cancer (ED SCLC) Pharmacoepidemiol Drug Saf. 2020 Dec 9

Part III Communication of real-world outcomes to patients

Chapter 8 Development and evaluation of a real-world outcomes based tool to support informed clinical decision-making in the palliative treatment of patients with metastatic non-small cell lung cancer submitted

General discussion

Summary

Appendices Samenvatting 

General introduction 



\section{Epidemiology and treatment of metastatic lung cancer}

Lung cancer is the second most commonly diagnosed cancer and the leading cause of cancer death worldwide [1]. In the Netherlands, more than 13,000 people are diagnosed with lung cancer annually [2]. Non-small cell lung cancer (NSCLC) is the most common type of lung cancer (85\%) and affects slightly more men than women. Small cell lung cancer (SCLC, 13\%) is equally common in men and woman. The incidence of NSCLC in the past decade increased, where the incidence of SCLC remained the same [2].

The majority of lung cancer patients is diagnosed with an advanced or metastatic disease $[1,3]$, and therefore has a poor prognosis. The overall 1-year survival rate of stage IV NSCLC or SCLC is only $23 \%$ and $21 \%$, respectively [2]. Illustrating the poor prognosis, median overall survival for untreated and systemically treated patients diagnosed with stage IIIB or IV NSCLC is approximately 2 and 10 months, respectively [4].

Palliative treatment for metastasized lung cancer consists of systemic treatment targeting tumour tissue (chemotherapy, targeted therapy and/or immunotherapy) or symptomatic relief by best supportive care (BSC). Numerous phase III trials have shown the superiority of systemic treatments over best supportive care in patients with metastatic lung cancer [5-7]. Platinum-based doublet therapy (for example, cisplatin in combination with another cytotoxic therapy) has been the standard therapy for patients with metastatic lung cancer for more than a decade [5].

In more recent years, treatment options for lung cancer have expanded with the arrival of targeted therapies and immunotherapies, with promising results [5, 8]. Targeted agents for patients with epidermal growth factor receptor (EGFR) mutations and anaplastic lymphoma kinase (ALK) rearrangements have proved large benefits in a small number of patients with NSCLC. More recently, immunotherapeutic agents directed on the programmed cell death-1 (PD-1) receptor or its ligand PD-L1 have demonstrated a moderate benefit but durable responses for a small number of patients with advanced NSCLC [9].

\section{Generalizability of clinical trials on systemic treatment}

However, data from clinical trials, although providing critical evidence of clinical activity, may not be reflective of treatment benefit and risk in all patient populations that will actually be treated in real-world practice. Due to strict patient inclusion criteria, important patient characteristics predictive for treatment response are often underrepresented in clinical trials [10-12]. The benefits and risks from drugs vary from one patient to the next, and are dependent on the treatment scenario [13]. Therefore, outcomes observed for patients selected to participate in clinical trials (efficacy) might differ from outcomes when the same drug or intervention is used in real-world circumstances (effectiveness); a so-called efficacy-effectiveness (EE) gap. The EE gap translates to inferior outcomes (e.g., disease response or survival) and greater toxicity when treatments are applied in a real-world clinical situation [12]. 
The concept of an EE gap applies to clinical trials across all diseases, but it is particularly relevant to patients with cancer [12]. For example, Templeton et al. showed that metastatic castrate-resistant prostate cancer trial patients treated with docetaxel are both younger and have a better performance status compared to patients treated in routine care [14]. Moreover, the median survival was 20.4 months for trial patients, whereas patients in routine care showed a median survival of only 13.6 months. Also toxicity was far more often present in routine care than in the clinical trial population.

Likewise, an EE gap in patients with lung cancer is conceivable. For example, NSCLC patients with a poor performance status are often not eligible for clinical trials [15]. Furthermore, it is known that elderly patients (aged $\geq 70$ years) are underrepresented in clinical trials evaluating treatments for NSCLC, despite the high incidence of lung cancer in the elderly [16-18]. This results in uncertainty about how results from clinical trials on lung cancer translate to the real-world population. Clinical trial data obtained in a younger population cannot be automatically extrapolated to the great majority of non-selected elderly patients with NSCLC, given the differences in comorbidity and performance status $[18,19]$.

\section{Efficacy-effectiveness gap and real-world data in lung cancer}

Some efforts have been made to provide insight into treatment outcomes in real-world populations with lung cancer versus clinical trials. For example, Zhu et al. [20] showed a shorter median survival estimate in routine practice for patients treated with bevacizumab-carboplatin-paclitaxel compared to participants in the ECOG 4599 trial: 9.7 months versus 12.3 months, respectively. A Canadian study showed that OS of patients receiving erlotinib in clinical practice was shorter than that reported in the pivotal clinical trial (5.19 vs. 6.70 months) [21]. A systematic literature review on real-world effectiveness of SCLC treatments suggested that an efficacy-effectiveness gap is also present in SCLC [22]. However, outside the few single treatment comparisons, a comprehensive overview of the possible divergence between clinical trials and routine clinical practice is still lacking for lung cancer [23]. Furthermore, individual patient data level studies exploring characteristics associated with a possible efficacy-effectiveness gap are missing [22].

\section{Clinical impact of real-world outcomes data}

Besides the identification of an EE gap for the re-evaluation of approved systemic treatments by payers and policy makers, real-world data is important to provide more appropriate information for patients regarding the effectiveness and toxicity of systemic therapies [10, 12]. Most oncologists use survival and toxicity data from clinical trials to counsel patients, inform decision-making, and obtain consent for treatment [24]. However, if an EE gap is demonstrated in patients with metastatic lung cancer, oncologists treating patients in routine clinical practice should have the appropriated evidence from real-world settings on which to base their clinical decisions and to provide information regarding clinical effectiveness in everyday practice [10]. Research has shown that oncologists tend 
to overestimate life expectancy of patients, and that they could benefit from using tools providing individual survival estimates [25]. The potential added value of providing real-world outcomes data through a tool has not been studied before in patients with metastatic NSCLC. At present, there is no decision support systems that gives an overview of all treatments relevant to consider in incurable NSCLC patients, or that offers information about intensive treatment versus best supportive care based on real-world data [26].

\section{Aim of this thesis}

The overall aim of this thesis is to assess how clinical outcomes in real-world (effectiveness) correspond to the outcomes in clinical trials (efficacy) of systemic treatments for metastasized lung cancer. Furthermore, to look into factors that may explain an EE gap and to develop and evaluate a tool for informed clinical decision-making in the palliative setting founded on real-world results.

\section{Outline of this thesis}

After the General introduction, this thesis is subdivided into three parts. In Part I, five studies are presented that investigate real-world outcomes of systemic treatment in stage IV NSCLC. Part II addresses the real-world outcomes in extensive disease SCLC, and Part III describes the information provision of real-world outcomes to patients and thoracic oncologists using a web-based tool.

\section{Part I - Real-world outcomes NSCLC}

For investigating real-world outcomes in lung cancer, it is necessary to determine which outcomes are relevant to patients and other stakeholders. Although efforts to report outcomes of lung cancer treatment in the Netherlands were present, there is no national standardized approach to report outcomes of all aspects of lung cancer treatment (including systemic treatment and best supportive care). Therefore, in Chapter 1 we provide information about the development of a set of outcome indicators to improve patient relevant outcomes for patients with lung cancer, as conducted in the Santeon 'Care for Outcomes' (CfO) project. Chapter 2 describes trends in systemic treatment and overall survival for NSCLC stage IIIB/IV using data from the Santeon CfO project and from the Santeon Farmadatabase, which comprises all prescribed and dispensed drugs at the individual patient level for all patients receiving care in one of the Santeon hospitals. Next to these real-world data, insight into treatment outcomes in clinical trials is necessary to explore the effectiveness of systemic treatment in real-world practice in relation to efficacy data from clinical trials (efficacy-effectiveness gap). Therefore, Chapter 3 presents an evaluation of the EE gap in patients with metastatic NSCLC by providing a systematic literature search and meta-analysis to obtain pooled efficacy results from clinical trials for first-line treatment regimens, compared to the obtained real-world data. Accordingly, the efficacy-effectiveness factor is introduced in this chapter, which reflects on how an 
individual survival outcome relates to the median overall survival (OS) from (the) corresponding clinical trial(s) for a first-line regimen.

As seen with the Value-Based Health Care concept, patient value is defined as a ratio between patient-relevant outcomes and costs. In addition to merely report on real-world outcomes, Chapter $\mathbf{4}$ describes the development of real-world systemic treatment costs over the years compared with changes in OS at the level of a diagnosis of stage IV NSCLC. As new treatment options emerged in more recent years (after the CfO project), real-world data on immunotherapy in metastasized NSCLC is presented in Chapter 5, including hazard ratios for patients with first-line pembrolizumab or second-line nivolumab in real-world versus clinical trials with the respective treatment.

\section{Part II - Real-world outcomes SCLC}

The aim of Part II of this thesis was to assess real-world outcomes in extensive disease (ED) SCLC. Chapter 6 describes real-world treatment patterns and outcomes of this patient population. Insight in treatment patterns is helpful to payers when considering the budget impact (e.g. expected patient volume) of novel treatment options when they come available, and as background for evaluation of the added value of future drugs under study. Subsequently, Chapter 7 evaluates the EE gap of systemic treatments in ED SCLC, by assessing how real-world outcomes of these patients correspond to the outcomes in clinical trials and which explanatory factors may explain a gap.

\section{Part III - Communication of real-world outcomes to patients}

Part III consists of a study on the development and evaluation of a web-based tool to provide insight in the real-world effectiveness of systemic treatments for patients with stage IV NSCLC ('patients like me' concept). In Chapter 8 , the real-world data from Part I is used to develop a tool in consultation with patients and thoracic oncologists. The tool is evaluated in a pilot study to assess the added value (usefulness and appreciation) in routine clinical practice.

Lastly, in the General discussion, the main findings of this thesis will be discussed and implications for future research and clinical practice will be outlined. 


\section{REFERENCES}

1. Siegel, R.L., K.D. Miller, and A. Jemal, Cancer statistics, 2020. CA Cancer J Clin, 2020. 70(1): p. 7-30.

2. Netherlands Comprehensive Cancer Organization. Netherlands Cancer Registry. Available from: www.cijfersoverkanker.nl. Accessed July 7, 2020.

3. van der Drift, M.A., et al., Progress in standard of care therapy and modest survival benefits in the treatment of non-small cell lung cancer patients in the Netherlands in the last 20 years. J Thorac Oncol, 2012. 7(2): p. 291-8.

4. Peters, B.J.M., et al., Trends in prescribing systemic treatment and overall survival for non-small cell lung cancer stage IIIB/IV in the Netherlands: 2008-2012. Cancer Epidemiol, 2017. 51: p. 1-6.

5. Herbst, R.S., D. Morgensztern, and C. Boshoff, The biology and management of non-small cell lung cancer. Nature, 2018. 553(7689): p. 446-454.

6. Molina, J.R., et al., Non-small cell lung cancer: epidemiology, risk factors, treatment, and survivorship. Mayo Clin Proc, 2008. 83(5): p. 584-94.

7. Non-Small Cell Lung Cancer Collaborative Group, Chemotherapy and supportive care versus supportive care alone for advanced non-small cell lung cancer. Cochrane Database Syst Rev, 2010(5): p. CD007309.

8. Lim, S.M., M.H. Hong, and H.R. Kim, Immunotherapy for Non-small Cell Lung Cancer: Current Landscape and Future Perspectives. Immune Netw, 2020. 20(1): p. e10.

9. Naylor, E.C., J.K. Desani, and P.K. Chung, Targeted Therapy and Immunotherapy for Lung Cancer. Surg Oncol Clin N Am, 2016. 25(3): p. 601-9.

10. Kennedy-Martin, T., et al., A literature review on the representativeness of randomized controlled trial samples and implications for the external validity of trial results. Trials, 2015. 16: p. 495

11. Rothwell, P.M., External validity of randomised controlled trials: "to whom do the results of this trial apply?". Lancet, 2005. 365(9453): p. 82-93.

12. Templeton, A.J., C.M. Booth, and I.F. Tannock, Informing Patients About Expected Outcomes: The Efficacy-Effectiveness Gap. J Clin Oncol, 2020. 38(15): p. 1651-1654.

13. Eichler, H.G., et al., Bridging the efficacy-effectiveness gap: a regulator's perspective on addressing variability of drug response. Nat Rev Drug Discov, 2011. 10(7): p. 495-506.

14. Templeton, A.J., et al., Translating clinical trials to clinical practice: outcomes of men with metastatic castration resistant prostate cancer treated with docetaxel and prednisone in and out of clinical trials. Ann Oncol, 2013. 24(12): p. 2972-7.

15. Al-Baimani, K., et al., Are clinical trial eligibility criteria an accurate reflection of a real-world population of advanced non-small-cell lung cancer patients? Curr Oncol, 2018. 25(4): p. e291-e297.

16. Blanco, R., et al., A review of the management of elderly patients with non-small-cell lung cancer. Ann Oncol, 2015. 26(3): p. 451-63.

17. Pallis, A.G., et al., Management of elderly patients with NSCLC; updated expert's opinion paper: EORTC Elderly Task Force, Lung Cancer Group and International Society for Geriatric Oncology. Ann Oncol, 2014. 25(7): p. 1270-1283.

18. Sacher, A.G., et al., Elderly patients with advanced NSCLC in phase III clinical trials: are the elderly excluded from practice-changing trials in advanced NSCLC? J Thorac Oncol, 2013. 8(3): p. 366-8.

19. Gridelli, C., et al., Treatment of advanced non-small-cell lung cancer in the elderly. Lung Cancer, 2009. 66(3): p. 282-6.

20. Zhu, J., et al., Carboplatin and paclitaxel with vs without bevacizumab in older patients with advanced non-small cell lung cancer. JAMA, 2012. 307(15): p. 1593-601.

21. Sheikh, N. and C.R. Chambers, Efficacy vs. effectiveness: erlotinib in previously treated non-small-cell lung cancer. J Oncol Pharm Pract, 2013. 19(3): p. 228-36.

22. Povsic, M., et al., Real-world effectiveness and tolerability of small-cell lung cancer (SCLC) treatments: A systematic literature review (SLR). PLoS One, 2019. 14(7): p. e0219622.

23. Peters, B.J.M., et al., Quantitative and qualitative assessment of real world data comparative effectiveness research of systemic therapies in lung oncology: A systematic review. Cancer Epidemiol, 2016. 44: p. 5-15.

24. Phillips, C.M., et al., Assessing the efficacy-effectiveness gap for cancer therapies: A comparison of overall survival and toxicity between clinical trial and population-based, real-world data for contemporary parenteral cancer therapeutics. Cancer, 2020. 126(8): p. 1717-1726. 
14 | GENERAL INTRODUCTION

25. Revesz, D., et al., Needs with Regard to Decision Support Systems for Treating Patients with Incurable Non-small Cell Lung Cancer. J Cancer Educ, 2020. 35(2): p. 345-351.

26. Revesz, D., et al., Decision support systems for incurable non-small cell lung cancer: a systematic review. BMC Med Inform Decis Mak, 2017. 17(1): p. 144. 
GENERAL INTRODUCTION $\mid 15$ 


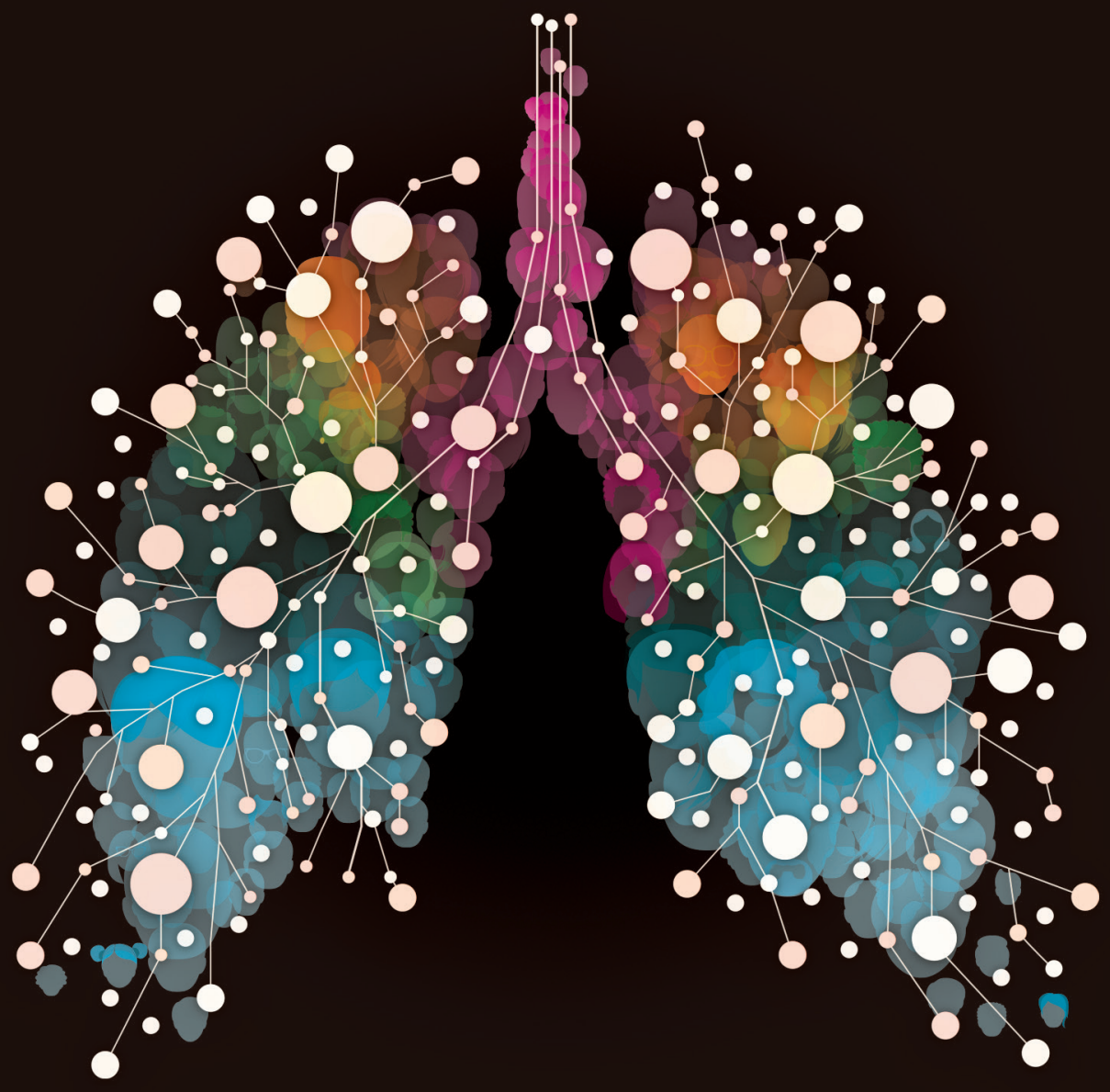


PART ।

Real-world outcomes NSCLC 



\section{Chapter 1}

'Care for Outcomes': systematic development of a set of outcome indicators to improve patient-relevant outcomes for patients with lung cancer

Christine M. Cramer-van der Welle, Lotte van Loenhout, Ben E.E.M. van den Borne, Franz M.N.H. Schramel, Lea M. Dijksman 


\section{ABSTRACT}

Objectives: Measuring quality of care is important, however many of the quality indicators used do not focus on outcome of treatment and aspects which are valuable for patients and physicians. The project 'Care for Outcomes' aims to establish a relevant set of outcome indicators for lung cancer.

Setting: Network of seven large, non-university teaching hospitals in the Netherlands (Santeon).

Methods: By reviewing literature, a list of potential outcome indicators for patients with lung cancer was composed and subsequently prioritised by expert's opinion. Three external parties, with expertise on lung cancer, clinical management, and public health evaluated and reduced the list of indicators to a working set. Finally, the resulting selection of outcome indicators was tested for feasibility and discrimination in patient data, by collecting retrospective data and performing regression and survival analyses.

Participants: Development of the indicator set in six Santeon hospitals. Retrospective cohort study in 5922 patients diagnosed with lung cancer (all types and stages).

Results: Selected outcome indicators were divided in three levels of outcome (tiers).

The first tier about survival and the process of recovery include mortality, survival, positive resection margins, rethoracotomy after resection, and quality of life at baseline and after 3, 6 and 12 months. Tier 2 concerning the sustainability of the recovery include complications after resection and toxicity after chemotherapy and/or radiation. Tier 3 about sustainability of health revealed no measurable outcomes. The retrospective data collection showed differences between hospitals and variation in case mix.

Conclusion: A relevant set of outcome indicators for lung cancer was systematically developed. This set has the potential to compare quality of care between hospitals and inform patients with lung cancer about outcomes. The project is ongoing in the current Santeon Value-Based Health Care programme through quality and improvement cycles. 


\section{INTRODUCTION}

Lung cancer is the most commonly diagnosed cancer and the leading cause of cancer death worldwide1. In the Netherlands, more than 13,000 people are diagnosed with lung cancer annually2. There are two main types of lung cancer: non-small cell lung cancer (NSCLC), which is the most common type (85\%), and small cell lung cancer (SCLC). The majority of patients is diagnosed with an advanced stage lung cancer (stage III or IV) ${ }^{3}$, and therefore has a poor prognosis. For example, median overall survival is approximately 2 and 10 months, for untreated and systemically treated patients diagnosed with stage IIIB or IV lung cancer, respectively4.

Significant regional differences and between-hospital variation in treatment patterns and outcomes for patients with NSCLC are shown in the Netherlands ${ }^{5}$. For example, substantial variation in time to treatment exists between patients and between hospitals for patients with advanced NSCLC6 ${ }^{6}$. Von Meyenfeldt et al. found a clinically relevant between-hospital variation in length of stay after lung cancer surgery?

Besides variation in treatment practices, quality indicators, which are commonly used to evaluate healthcare and identify areas for improvement, also vary across hospitals. Healthcare institutions often register a wide set of indicators; however, many indicators focus on the process and structure of the treatment, rather than on its outcomes.

For instance, the time required to perform a CT scan on a patient with a pulmonary mass is a commonly used process indicator. However, while such an indicator might signal any number of issues in the diagnostic process, it is not usually relevant to the eventual outcome of the patient. Registering these kinds of indicators burdens doctors with administrative tasks that do not necessarily have an impact on relevant health outcomes for patients. Similarly, if hospitals register different sets of indicators to measure their quality of care, this will limit supervisory institutions' ability to gain insight into the quality of care. Value-Based Health Care (VBHC) is increasingly being promoted as a strategy for improving quality of care. Based on the principles formulated by Porter 89 , value is defined as patientrelevant health outcomes per dollar spent for a specific medical condition over the full cycle of care. The first step is to define outcomes that matter to patients and other stakeholders. Relevant measures of quality would reflect the survival and degree of recovery, process of recovery (e.g. treatment related complications), and sustainability of recovery. Furthermore, these measures of quality should be collected in a standardized way. Although efforts to report outcomes of lung cancer treatment exist, for example, the Dutch Lung Cancer Audit for Surgery (DLCA-S)10 11, there is no national standardized approach to report outcomes of all aspects of lung cancer treatment (including systemic treatment and best supportive care). Additionally, patients' reports of their health-related quality of life (QoL) are rarely measured routinely. Furthermore, true comparison would only be possible when patient groups are relatively homogeneous or when correction for case mix (combination of patient and disease characteristics) is reliably achieved. 
This article discusses the project 'Care for Outcomes' (CfO)'12, which aims to develop a relevant set of outcome indicators for lung cancer, including patient-reported outcome measures (PROMs) and case-mix factors to increase the usefulness of comparisons across treatment modalities and institutions.

\section{METHODS}

\section{Outcome indicator selection process}

An indicator selection process was performed in three phases in order to determine a set of outcome indicators (Figure 1). The initial broad selection is based on a review of the literature and prioritizing by expert opinion (Phase I). Subsequently, external stakeholders evaluated and reduced the list of indicators to a working set (Phase II). Finally, the resulting selection of outcome indicators was tested for feasibility and discriminative aspects in patient data (Phase III).

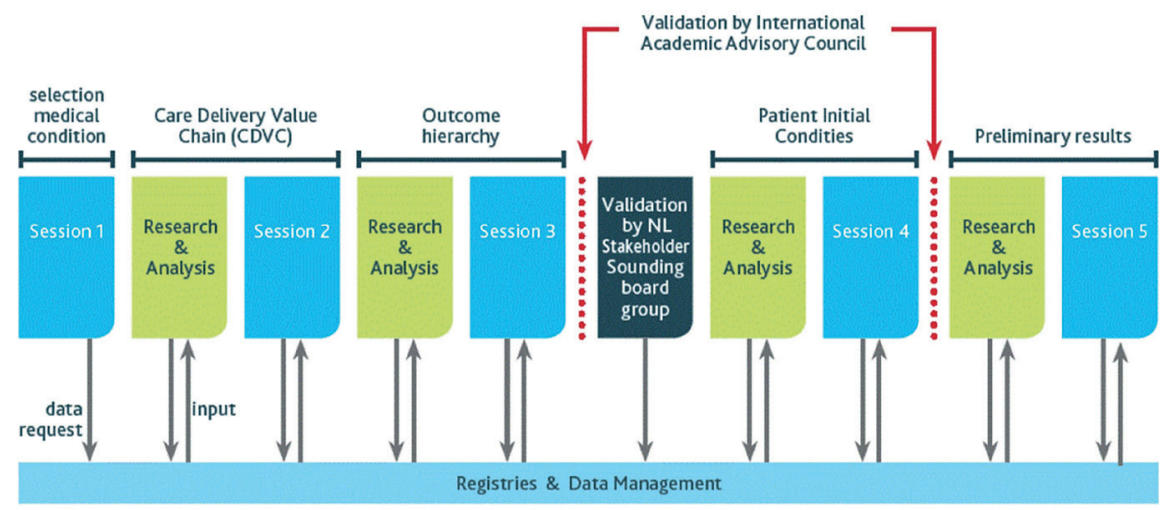

Figure 1. Different steps in the development of outcome indicators in the project 'Care for Outcomes'

\section{Phase I: literature review and classification of potential outcome indicators}

First, a literature review was conducted for defining potential outcome indicators. The goal of this search was not to identify all available relevant literature, but to obtain a comprehensive overview of all possible and relevant outcome indicators that could act as a starting document for the discussion between different caregivers. The search included all types and stages of lung cancer. A 'care delivery chain' was formulated in order to organize potential indicators. This chain lists all activities in the process of care. ${ }^{8}$ By distinguishing individual activities, it is possible to identify which of them create value for the patient. 
The potential outcome indicators as derived from the literature were divided into three tiers according to the outcome hierarchy by Porter (Appendix Figure 1). The first tier includes survival and degree of recovery, tier 2 relates to the process of recovery, and the third tier comprises the sustainability of recovery. Next, a team of pulmonologists, nurse specialists, and policy makers, prioritized systematically the selected possible outcome indicators from the literature. Therefore, a Delphi process - an established method of achieving a consensus between experts - was used. ${ }^{13}$ Every indicator was evaluated according to: a) the relevance to the patient; b) the medical relevance, i.e. the extent to which the indicator measures the quality of care; and c) the relevance for patient populations, for example the more prevalent complications are considered to be more relevant. Missing indicators could be added on the list by team members. All indicators with an SD of the ranking of more than 1.5 , or added by one of the members, were discussed. With this list, the top 2-3 outcome measures per subtier were selected.

\section{Phase Il: evaluation and reduction of indicators by national and international stakeholders}

In the second phase of the selection, every indicator that passed the first phase was evaluated by three external parties. An international academic council consisting of experts in lung cancer evaluated the medical relevance of the selected indicators. A methodology academic advisory council with experts on clinical management, public health and decision sciences assessed the methodology of the project. And thirdly, an advisory board consisting of representatives of patient associations, health insurers and the government gave more general advice on the process, based on social relevance. This second phase in the selection process resulted in a working set of outcome indicators.

\section{Phase III: testing the feasibility and discriminative aspect of the indicator set in clinical practice}

The third and final phase of the indicator selection process involved the testing of the indicator set in practice, in a retrospective data analysis based on data of patients in the Santeon hospitals, a network of seven large, non-university teaching hospitals in the Netherlands. First, the distinctive power, the comparability and the reproducibility of the selected indicators was tested in patient data collected retrospectively for the years 2008-2011 in two Santeon hospitals, the St. Antonius Hospital in Nieuwegein/Utrecht and Catharina Hospital in Eindhoven. ${ }^{14}$ After this first round of data collection, the quality and feasibility of data collection was scored. The outcomes of this analysis were shared with the three external parties (councils and advisory board), who re-evaluated the indicators based on the results of the first round. The final set of outcome indicators was selected for analysis based on the empirical results and the re-evaluation by external parties.

Second, the patient data collection was extended to four other Santeon hospitals, in which two rounds of data collection were performed (round 1: data for the years 2008-2012, and round 2: data for the years 2008-2014).12 15 These data were analysed regarding the differences in outcomes when taking case-mix variables into account. 


\section{Statistical analysis}

Descriptive statistics were used for evaluating the data collected in Phase III of the project. Multivariable survival analysis was conducted to assess the discriminative potential of the selected case mix variables. The statistical analysis was performed using IBM SPSS statistics V.21.0. Continuous data were expressed as mean \pm SD or median (range) when appropriate. Categorical data were analysed using Chi-square and continuous data using Student's t-test, rank tests and one-way Analysis Of Variance (ANOVA) when appropriate. A p-value of 0.05 or smaller was considered statistically significant.

Comorbidity was defined by the Charlson Comorbidity Index (CCI), which is a sum score based on the associated weight of each of the 17 comorbidities, and an indicator of disease burden.16-18 Feasibility is measured by calculating the percentage of patients for whom it was possible to collect the outcome indicator (non-missing).19

Next, a multivariable Cox-regression analysis was performed to find independent predictors of long-term survival. All available characteristics were explored as potential prognostic factors (Eastern Cooperative Oncology Group Performance Status (ECOG PS), age, gender, $\mathrm{CCl}$, coronary heart disease, diabetes, lung function percentage of forced expiratory volume in one second (FEV1\%) and percentage of carbon monoxide transfer factor (DLCO\%), and morphology of the tumour (NSCLC and SCLC)). Outcomes were presented in whisker plots. For each hospital a Cox regression was performed to compare the concerning hospital with the other five hospitals. Patients still alive at date of follow-up were censored and given the end of follow-up date as imputed date of death. Logistic regression was used to find independent predictors for outcomes such as complications after resection. In this analysis, missing values were imputed by giving the value 0 , which indicates that the patient did not have the characteristic.

\section{Patient and public involvement statement}

The goal of this study was to develop outcome indicators that are relevant for patients. All the participants were very aware of this goal and throughout the process the interests of the patients were therefore of special importance. The patients were not themselves involved in the design and conduct of the study. Nevertheless, patient contribution was established by including patient representatives in the advisory board. Dissemination of project results to patients is facilitated by infographics and a website.

\section{RESULTS}

\section{Phase I}

After reviewing the literature, a first set of 80 potential outcome indicators was selected. Subsequently, the team of pulmonologists, nurse specialists, and policy makers reduced the first set to 25 potential outcome indicators based on relevance. Finally, Phase I yielded 2-3 outcome measures per subtier. 


\section{Phase II}

The selection of Phase I was evaluated and further reduced by the three external parties in Phase II. This resulted in the working set of seven outcome indicators, which are shown in Table 1, per tier according to the outcomes hierarchy of Porter. The selected indicators are specific to the treatment, such as the share of positive resection margins, which signals the quality of surgical treatment. The QoL questionnaires used were the European Organization for Research and Treatment for CancerQuality of Life Questionnaire (EORTC QLQ) C30 and LC13, which are the most commonly used and validated questionnaires for these type of patients. Tier 3 revealed no measurable outcomes, because they did not meet the criteria of adding value to the patient, medical relevance and relevance to patient populations.

Besides the selected outcome indicators from the CfO Project, indicators from the International Consortium of Health Outcomes Measurement (ICHOM) Standard Set for Lung Cancer are also shown in Table 1, which adds to discussion about the possibilities for benchmarking outcome measures in lung cancer worldwide. Furthermore, descriptive results from Phase III (retrospective data collection) were shown regarding the number of patients involved for each outcome measure and the feasibility of the data (percentage non-missing data).

\section{Phase III}

In Phase III, data of 5922 patients diagnosed between 2008 and 2012 in the Santeon hospitals were analysed. Baseline characteristics and outcome data can be found in the CfO result book,12 and Appendix Table 1. The data show high feasibility scores around 90\%-99\% (Table 1), except for PROMs data, which were measured in a different cohort because of the prospective nature. Additionally, the response rate on PROMs deteriorated quickly after baseline measurement among patients with stage IV lung cancer (Figure 2). Figure 3 shows that the largest group of patients $(61 \%, n=3612)$ had advanced lung cancer (stage IIIB or IV), with some differences between hospitals (range 56\%-64\%). These patients received palliative treatment (systemic treatment or best supportive care). Patients with stages I-IIIA lung cancer received curative treatment with resection, radiotherapy and/or chemotherapy.

To illustrate between-hospital comparisons and the relevance of the set in clinical practice, the results concerning survival after resection in patients with lung cancer stage I-IIIA are shown in Figure 4. In this analysis, case-mix characteristics were taken into account. A multivariable regression analysis showed that stage, ECOG PS and age, were the strongest predictors of survival after resection (Figure 4). Those characteristics were comparable across hospitals, although ECOG shows some variation in the distribution between ECOG 0 or 1 (ECOG 0: range 29\%-46\%; ECOG 1: range 24\%-44\%; resulting in a range of 58\%-74\% of the patients with ECOG 0-1 across hospitals) and the percentage of unknown ECOG 


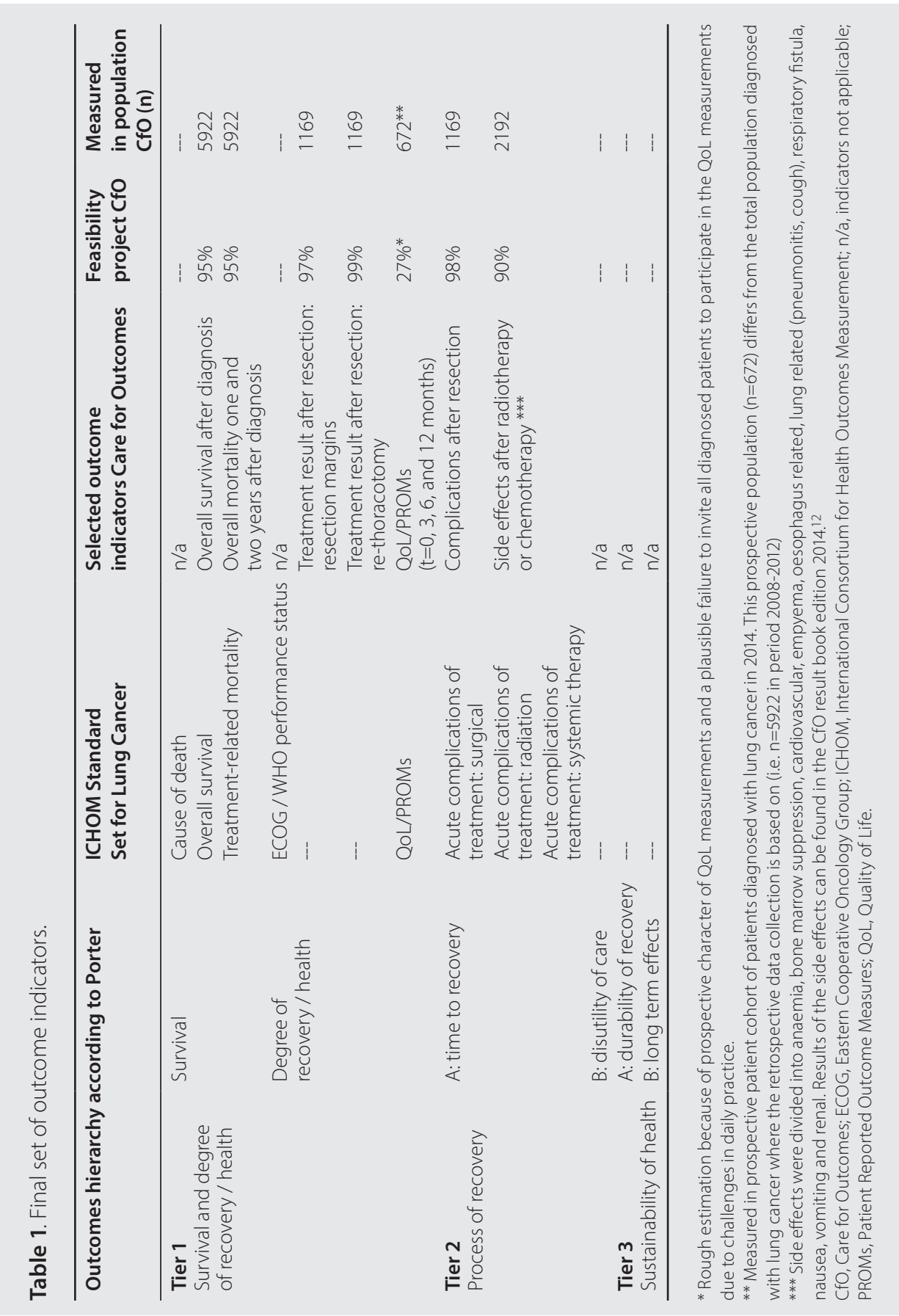




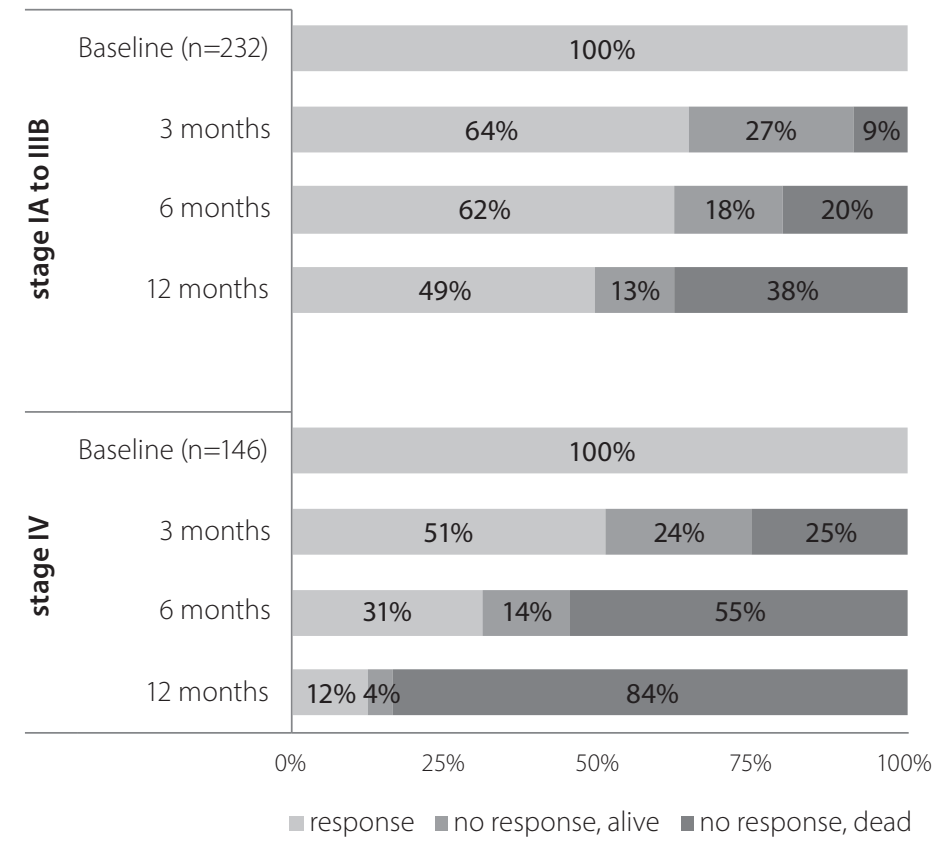

Figure 2. Patient-reported outcome measures response rates after baseline for patients with lung cancer stage IA-IIIB and IV.

values (2\%-21\%). After adjustment for case-mix variables using a Cox proportional hazard model, all hospitals showed comparable rates of mortality for patients with lung cancer stages I-IIIA and resection.

For patients with lung cancer stages I-IIIA without resection, analysis showed that one hospital showed a significantly better survival compared with the other hospitals. This result was used for further analysis for 'best practice', because it could be the effect of differences in the administration of chemoradiation therapy in each hospital. A team of healthcare professionals looked at these data and the corresponding method of practice in each hospital in detail and compared those to find possible differences. The survival data showed a significant better survival for patients aged $<75$ years who were treated with concurrent chemoradiation, compared with sequential administration of chemotherapy and radiation. For patients aged 75 years and older, no significant effect on survival was found between concurrent and sequential chemoradiation. ${ }^{15}$ This finding led to further research questions, because concurrent chemoradiation is associated with more adverse side effects.

The final set of outcome indicators was used in both rounds of data collection (2008-2012 and 2008-2014) in the CfO Project. Adjustment of the set followed in the new Santeon 


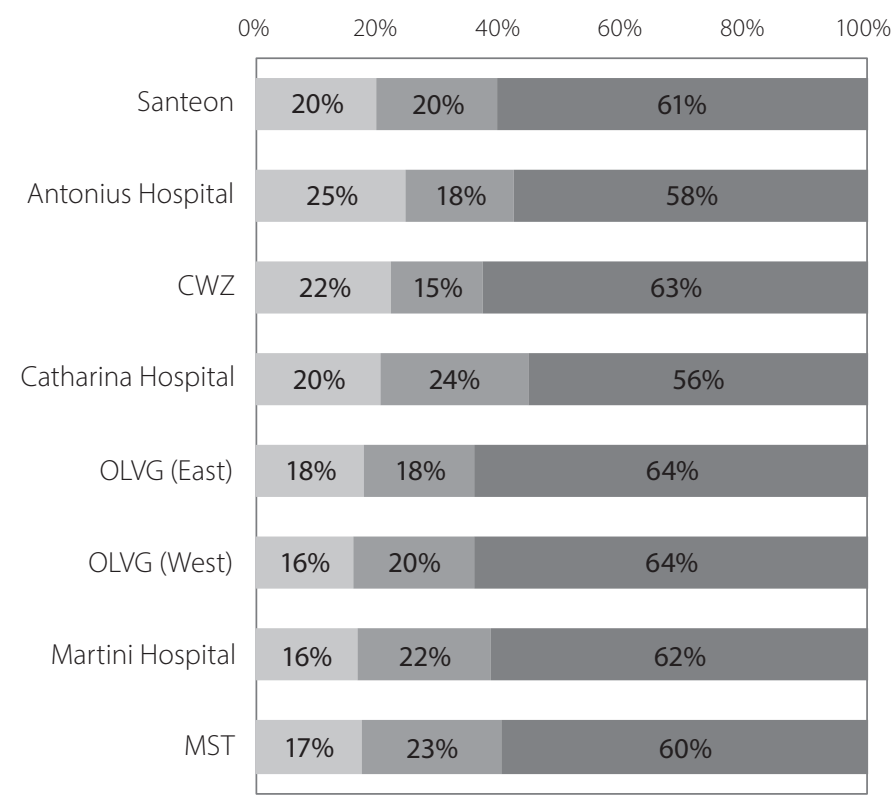

mstage IA - IIIA resection stage IA - IIIA no resection astage IIIB - IV

Figure 3. Distribution of stage and treatment in patients with lung cancer from Care for Outcomes database. CWZ, Canisius Wilhelmina Ziekenhuis; MST, Medisch Spectrum Twente; OLVG, Onze Lieve Vrouwe Gasthuis.

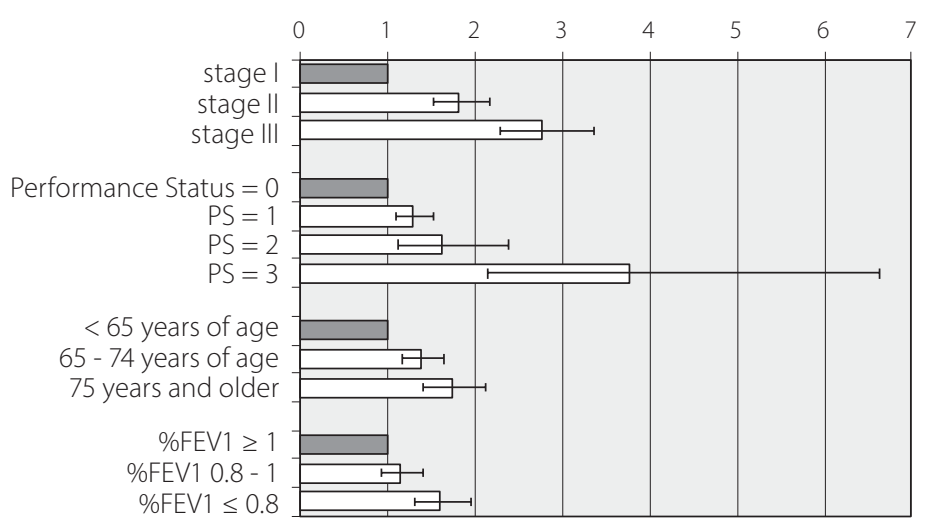

Figure 4. Influence of the most important case-mix variables on survival after resection ( $\mathrm{HR}$ and 95\% Cl) 
programme ' $\mathrm{VBHC}$ ', which is the continuation to the CfO project. At present, the set of indicators is in a constant process of review and is being evaluated every cycle (6 months) of the VBHC programme.

\section{DISCUSSION}

The aim of the CfO Project was to obtain a compact set of indicators to evaluate the quality of care in lung cancer treatments across the whole value chain. The project involves input from several external parties to ensure that medical, methodological, and ethical aspects of the selection process are addressed. The obtained set of outcome indicators for patients with lung cancer, which encompasses the whole process of care, is novel and unique to the Netherlands. The resulting set of indicators consists of only six variables from three different outcome hierarchy levels: survival (mortality and median survival after diagnosis); degree of recovery (treatment result after resection and QoL), and process of recovery (complications after resection and side effects after radiotherapy or chemotherapy). These indicators, tested for their feasibility and discriminative aspect to patient outcomes, differ from most quality indicators nationwide, which focus on the process and structure of care or on only a part of clinical care for patients with lung cancer. For example, a review by Numan et al. ${ }^{20}$ identified five evidence-based quality indicators for stages I-III NSCLC, but only focused on preoperative and postoperative care.

This study showed that the introduced set of outcome indicators seemed feasible to collect data from medical charts retrospectively, with few missing data. Furthermore, the set is sensitive to detect differences in outcomes between hospitals. The administrative burdens of gaining insight into the quality of care can thus be reduced when using such a smaller set of indicators than is currently used in many hospitals.

Alongside the established set of outcome indicators, patient and disease characteristics (case-mix variables) showed to be important in collecting data for comparisons across treatment modalities and hospitals. One of the analyses in Phase III of this CfO Project showed that a patients' age at diagnosis is significantly associated with survival in patients with stages I-IIIA lung cancer. Existing literature about age as predictor for survival in lung cancer is inconclusive, with differences over time and in stages of disease. ${ }^{3} 2122$ These different findings could be the result of, for instance, the population sample of patients used in the analysis, relations with other predictive factors (e.g. ECOG PS and CCI), or differences between hospitals in accompanying (symptom-related) treatments. These findings emphasize the importance of proper adjustment when comparing hospitals on outcome indicators. Additionally, when analysing patient outcomes and benchmarking between hospitals, it is important to look beyond significant differences and use 
differences in outcomes with substantial clinical impact in the ongoing debate about improving quality of care. Furthermore, it remains essential to provide lung oncologists and other caregivers their own unadjusted data, which could promote opportunities to improve care processes.

A strength of this study is the combination of a structured development and a hands-on validation of an outcome indicator set in a large unselected cohort of patients, sized around $12 \%$ of the Dutch population with lung cancer. Therefore, the cohort presented in this study can be found as reppresentation of current national practice. Furthermore, the established set of indicators seemed feasible to collect data from medical charts retrospectively with few missing data (high feasibility rates). Finally, the set is sensitive to detect differences in outcomes between hospitals in order to fuel discussions towards improvements in delivery of care.

Our approach has some limitations that should be considered. First, although the established indicator set for lung cancer reflects literature reviews and feedback among a relevant team of experts, representatives of patients associations, and other stakeholders, the suggested outcomes and measures remain expert's opinion. Additionally, no outcomes measures were specified in the third tier about sustainability of health or recovery and long-term consequences of therapy, since the potential outcome indicators in tier $3 \mathrm{did}$ not meet the criteria for relevance according to the experts. However, the development of the set was defined as a starting point to obtain a compact set of indicators towards routine collection and evaluation of patient-centred outcomes for patients with lung cancer. The outcome indicator set is subject to discussion in a constant process of review with possible adjustments as the project evolves.

Second, the selected indicator set was tested on retrospective data. Therefore, the outcome set might not perfectly elucidate the current quality of care. Additionally, the CfO Project was faced with differences between hospitals in registration during the process of retrospective data collection (e.g., complications or ECOG PS). Studies on benchmarking between hospitals would benefit from a more systematic measurement across hospitals, which eventually may also benefit outcome comparisons in general.

Finally, prospective data collection on QoL through patient reported outcome measures was challenging. With the small number of responding patients to the QoL measurements (approximately $27 \%$ of patients), the question remains if the QoL results are valid and can be used in quality improvement. The Santeon hospitals are now working on improving the methods on PROM measurement for two problems experienced in the collection of QoL: 1) not every patient diagnosed with lung cancer received a QoL questionnaire, and 2) not every patient who fills in the baseline questionnaire, persevered in completing the QoL questionnaires at the successive measurement points. For patients with stage IV lung cancer, an explanation for the deteriorating response rates could be the poor prognosis among this group of patients. Shorter intervals between the surveys might improve the insights for such groups of patients. 
Currently, the outcome indicator set for lung cancer is in a constant process of review in the Santeon VBHC programme, within a 6 month evaluation cycle. In this process, a number of factors are important to assess whether an outcome indicator remains suitable for the outcome set, for example, the availability of data, the prevalence of the measured indicator (e.g. if a complication is rare, it is not a suitable indicator), and whether an indicator has a discriminative aspect (e.g. if outcomes of an indicator are the same in every hospital, it is not very informative).

Furthermore, the results of this project are used in the Santeon hospitals to inform patients about their treatment options, prognosis associated with specific treatments, and possible side effects. To facilitate patient understanding, typical outcomes were displayed in infographics. There is also a special website to inform patients about the project and its results. ${ }^{23}$ Hopefully, these insights support shared decision-making between patients and doctors when choosing treatment plans.

The data achieved by this project were used for several other studies.4 24-26 Additionally, the results of this project are associated with policy recommendations that apply to other fields of medicine. Similar projects were started within the Santeon hospitals to identify outcome indicators for breast cancer, colon cancer and prostate cancer. These are all aimed at eventually improving the quality of healthcare. This is being carried out through the establishment of quality cycles, which is an ongoing project within the current Santeon VBHC projects.

The CfO Project nowadays connects to several international projects on lung cancer, for example the UK National Lung Cancer Audit.27-31 Furthermore, ICHOM establishes sets of outcome indicators per disease in a similar systematic manner. ${ }^{32}$ It also employs experts and representatives of patient associations. Nowadays, a new initiative in the Netherlands, the DLCA, uses prospective registration and this is expected to increase transparency in lung cancer care.10 The DLCA now uses indicators that have been developed as part of the present project.

\section{CONCLUSION}

A relevant set of outcome indicators for lung cancer care is systematically developed in this CfO Project. The indicators are relevant to the patient as well as doctors, and are sensitive to detect differences in outcomes between hospitals in order to fuel discussions towards improvements in delivery of care. The goal of the Santeon hospitals is to inform patients about this process and the aim for improving care continuously. Insight in treatment options and expected outcomes can support shared decision-making. The project is ongoing in the Santeon VBHC programme, with continuously measuring outcomes and implementing improvements. Use of the Santeon set of outcome indicators 
and the resulting recommendations could also enable other institutions to monitor, compare and potentially improve the quality of their lung cancer care.

\section{ACKNOWLEDGEMENTS}

The authors thank the Project team 'Care for Outcomes': Judith Herder (St. Antonius Hospital; now: Meander Medisch Centrum, Amersfoort), René Termeer (Canisius Wilhelmina Hospital, Nijmegen), Albert Polman (Medisch Spectrum Twente, Enschede), John van Putten (Martini Hospital, Groningen), Arthur Smit (OLVG, Amsterdam), Arifa Moons-Pasic (OLVG, Amsterdam), Wim van den Bosch (St. Antonius Hospital), and Pauline Gantvoort (Santeon) for their efforts in developing the indicator outcome set and completion and validation of hospital data. Furthermore, we thank the members of the International Academic Council, Methodology Academic Advisory Council, and the advisory board, including the patient representatives.

\section{Ethical approval}

The study was approved by the Santeon Management committee. Further ethical approval was not necessary for this study at that time, as it did not require any patient intervention and because of the re-use of available data. The need for informed consent was waved because of anonymous data handling and the retrospective nature of the study. Patients in the prospective PROMs cohort gave their consent in the online PROMs environment, which stated that their completed forms could be used anonymously for benchmarking or research. 


\section{REFERENCES}

1. Bray F, Ferlay J, Soerjomataram I, et al. Global cancer statistics 2018: GLOBOCAN estimates of incidence and mortality worldwide for 36 cancers in 185 countries. CA Cancer J Clin 2018;68(6):394-424. doi: 10.3322/ caac.21492 [published Online First: 2018/09/13]

2. The Netherlands Cancer Registry. www.cijfersoverkanker.nl. Accessed 14/03/2019.

3. Driessen EJ, Aarts MJ, Bootsma GP, et al. Trends in treatment and relative survival among Non-Small Cell Lung Cancer patients in the Netherlands (1990-2014): Disparities between younger and older patients. Lung Cancer 2017;108:198-204. doi: 10.1016/j.lungcan.2017.04.005 [published Online First: 2017/06/20]

4. Peters BJM, Cramer-Vd Welle CM, Smit AAJ, et al. Trends in prescribing systemic treatment and overall survival for non-small cell lung cancer stage IIIB/IV in the Netherlands: 2008-2012. Cancer Epidemiol 2017;51:1-6. doi: 10.1016/j.canep.2017.08.001

5. Wouters MW, Siesling S, Jansen-Landheer ML, et al. Variation in treatment and outcome in patients with non-small cell lung cancer by region, hospital type and volume in the Netherlands. Eur J Surg Oncol 2010;36 Suppl 1:S83-92. doi: 10.1016/j.ejso.2010.06.020 [published Online First: 2010/07/06]

6. van de Ven M, Retel VP, Koffijberg H, et al. Variation in the time to treatment for stage III and IV non-small cell lung cancer patients for hospitals in the Netherlands. Lung Cancer 2019;134:34-41. doi: 10.1016/j.lungcan. 2019.05.023 [published Online First: 2019/07/20]

7. von Meyenfeldt EM, Marres GMH, van Thiel E, et al. Variation in length of hospital stay after lung cancer surgery in the Netherlands. Eur J Cardiothorac Surg 2018;54(3):560-64. doi: 10.1093/ejcts/ezy074 [published Online First: 2018/03/08]

8. Porter ME. What is value in health care? NEngl J Med 2010;363(26):2477-81. doi: 10.1056/NEJMp1011024

9. Porter ME, Larsson S, Lee TH. Standardizing Patient Outcomes Measurement. N Engl J Med 2016;374(6):504-6. doi: 10.1056/NEJMp1511701

10. DICA. Dutch Lung Cancer Audit. Available from: https://dica.nl/dlca/home accessed Feb 2019.

11. Ten Berge M, Beck N, Heineman DJ, et al. Dutch Lung Surgery Audit: A National Audit Comprising Lung and Thoracic Surgery Patients. Ann Thorac Surg 2018;106(2):390-97. doi: 10.1016/j.athoracsur.2018.03.049 [published Online First: 2018/04/25]

12. Santeon. Care for Outcome, Outcome indicators for cancer, Results Santeon Hospitals Edition 2014. Published April 2015. https://www.santeon.nl/Santeon_CareForOutcome2014.pdf.

13. Jones J, Hunter D. Consensus methods for medical and health services research. BMJ 1995;311(7001):376-80.

14. Santeon. Zorg voor Uitkomst, Uitkomstindicatoren voor prostaatkanker en longkanker; Resultaten Catharina Ziekenhuis, St. Antonius Ziekenhuis en Canisius-Wilhelmina Ziekenhuis, Edition 2013, published Feb 2014. https://www.santeon.nl/uitkomstenboek2013.pdf.

15. Santeon. Care for Outcomes, the outcome indicator results for cancer treatment at Santeon Hospitals Edition 2016, Nov 2016. https://www.santeon.nl/wp-content/uploads/2015/01/Care-for-Outcomes-2016.pdf.

16. Charlson ME, Pompei $\mathrm{P}$, Ales $\mathrm{KL}$, et al. A new method of classifying prognostic comorbidity in longitudinal studies: development and validation. J Chronic Dis 1987;40(5):373-83. doi: 10.1016/0021-9681(87)90171-8 [published Online First: 1987/01/01]

17. Sundararajan V, Henderson T, Perry C, et al. New ICD-10 version of the Charlson comorbidity index predicted in-hospital mortality. J Clin Epidemio/ 2004;57(12):1288-94. doi: 10.1016/j.jclinepi.2004.03.012 [published Online First: 2004/12/25]

18. Birim O, Maat AP, Kappetein AP, et al. Validation of the Charlson comorbidity index in patients with operated primary non-small cell lung cancer. Eur J Cardiothorac Surg 2003;23(1):30-4.

19. Report from the National Quality Forum: eMeasure Feasibility Assessment. https://www.qualityforum.org/ Publications/2013/04/eMeasure_Feasibility_Assessment.aspx.

20. Numan RC, Berge MT, Burgers JA, et al. Pre- and postoperative care for stage I-III NSCLC: Which quality of care indicators are evidence-based? Lung Cancer 2016;101:120-28. doi: 10.1016/j.lungcan.2016.05.022

21. Chen $T$, Zhou F, Jiang $W$, et al. Age at diagnosis is a heterogeneous factor for non-small cell lung cancer patients. J Thorac Dis 2019;11(6):2251-66. doi: 10.21037/jtd.2019.06.24 [published Online First: 2019/08/03] 
22. Eguchi T, Bains S, Lee MC, et al. Impact of Increasing Age on Cause-Specific Mortality and Morbidity in Patients With Stage I Non-Small-Cell Lung Cancer: A Competing Risks Analysis. J Clin Oncol 2017;35(3):281-90. doi: 10.1200/JCO.2016.69.0834 [published Online First: 2017/01/18]

23. Santeon. Kwaliteit van longkankerzorg. In: https://www.santeonvoorlongkanker.nl/media/52708/santeon infographic_longkanker_v4.pdf, ed. 2016.

24. Cramer-van der Welle CM, Peters BJM, Schramel F, et al. Systematic evaluation of the efficacy-effectiveness gap of systemic treatments in metastatic nonsmall cell lung cancer. Eur Respir J 2018;52(6) doi: 10.1183/13993003.01100-2018

25. Wolff HB, Alberts L, Kastelijn EA, et al. Differences in Longitudinal Health Utility between Stereotactic Body Radiation Therapy and Surgery in Stage I Non-Small Cell Lung Cancer. J Thorac Oncol 2018;13(5):689-98. doi: 10.1016/j.jtho.2018.01.021

26. Sluga R, Van den Borne BEEM, Roepman P, et al. Utilization of Molecular Testing and Survival Outcomes of Treatment with First- or Second-line Tyrosine Kinase Inhibitors in Advanced Non-small Cell Lung Cancer in a Dutch Population. Anticancer Res 2018;38(1):393-400. doi: 10.21873/anticanres.12235

27. Wang X, Su S, Li S, et al. Development of quality indicators for non-small cell lung cancer care: a first step toward assessing and improving quality of cancer care in China. BMC Cancer 2017;17(1):603. doi: 10.1186/s12885017-3602-0

28. Brunelli A, Refai M, Salati M, et al. Standardized combined outcome index as an instrument for monitoring performance after pulmonary resection. Ann Thorac Surg 2011;92(1):272-7. doi: 10.1016/j.athoracsur.2011.03.038

29. Mainz J, Hansen AM, Palshof T, et al. National quality measurement using clinical indicators: the Danish National Indicator Project. J Surg Oncol 2009;99(8):500-4. doi: 10.1002/jso.21192

30. Tanvetyanon T. Quality-of-care indicators for non-small cell lung cancer. Cancer Control 2009;16(4):335-41. doi: $10.1177 / 107327480901600408$

31. Royal College of Physicians. National Lung Cancer Audit 2015. Available from: https://www.rcplondon.ac.uk/ projects/national-lung-cancer-audit accessed Feb 2019

32. International Consortium for Health Outcomes Measurement. Available from: https://www.ichom.org/ accessed Feb 2019 


\section{APPENDIX CHAPTER 1}

Outcome hierarchy according to Porter

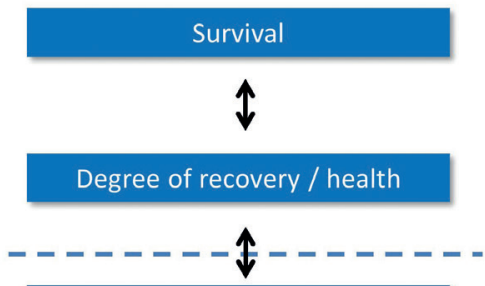

1. Survival and degree of recovery

Time to recovery or return to normal activities

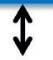

Disutility of care / complications

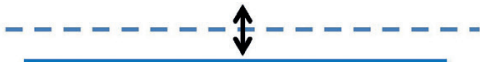

Sustainability of recovery or health over time

个

Long-term consequences of

therapy (e.g. care induced

illness)
2. Process of recovery

A time to recovery

B disutility of care: treatment related complications, adverse effects, treatment errors

3. Sustainability of health

Appendix Figure 1. Outcomes hierarchy according to Porter 
Appendix Table 1. Baseline characteristics population 'Care for Outcomes' 2014

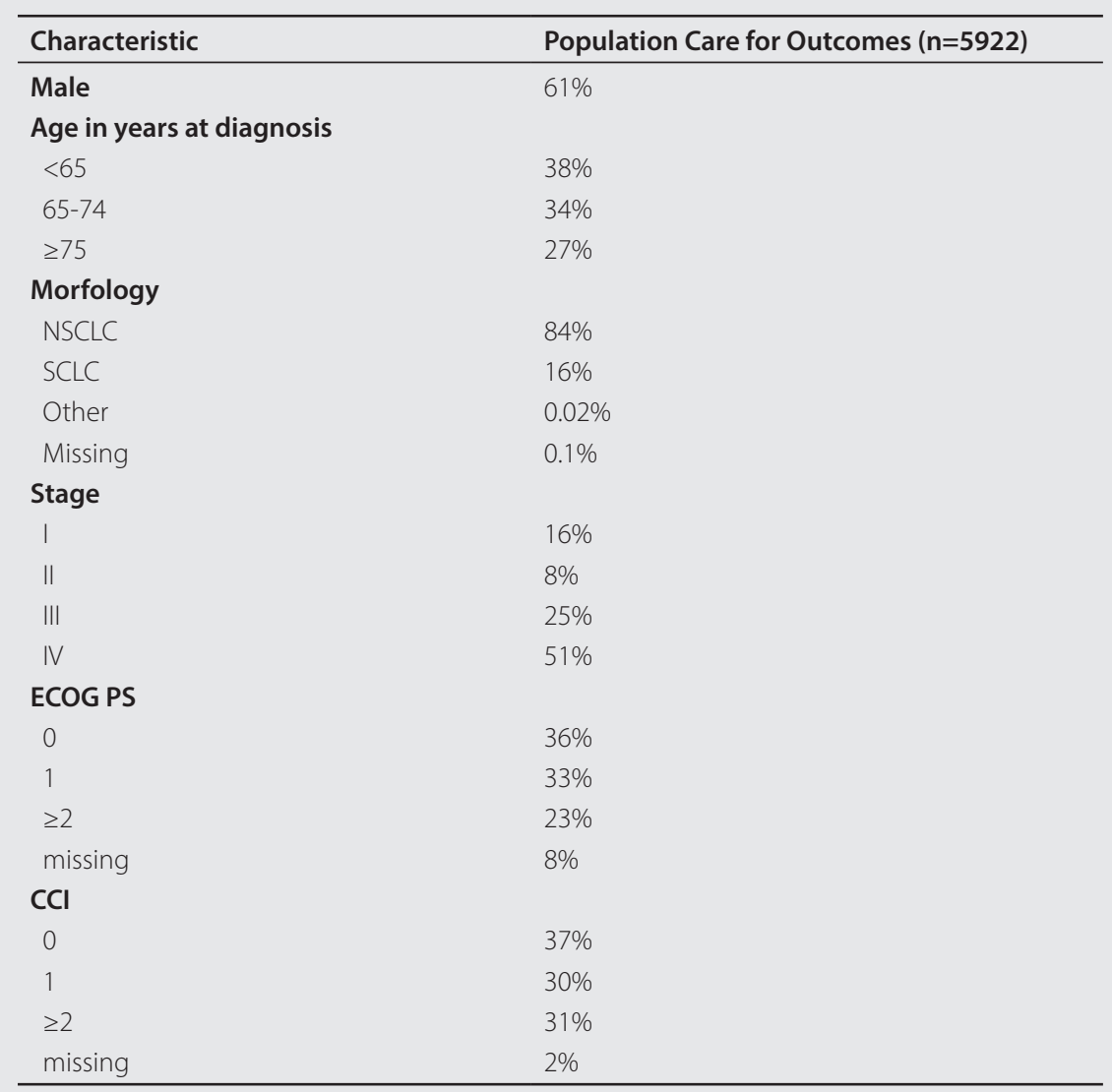

ECOG PS: Eastern Cooperative Oncology Group performance status; CCl: Charlson Comorbidity Index 



\section{Chapter 2}

Trends in prescribing systemic treatment and overall survival for non-small cell lung cancer stage IIIB/IV in the Netherlands: 2008-2012

Bas J.M. Peters, Christine M. Cramer-vd Welle, Arthur A.J. Smit, Franz M.N.H. Schramel, Ewoudt M.W. van de Garde, for the Santeon NSCLC Study Group 


\section{ABSTRACT}

Background: The present study aims to give a detailed overview of day-to-day practice in the systemic treatment of NSCLC stage IIIB/IV and its clinical outcomes in six large teaching hospitals in the Netherlands in the period 2008-2012.

Methods: A retrospective observational cohort study was conducted in the Care for Outcome registry. Patients diagnosed with stage IIIB/IV NSCLC were included and drug data were collected. Outcomes included percentage of patients treated with systemic treatment, percentage of different first-line treatment options, survival, and number and percentage of switches, dose reductions ( $<80 \%$ of the initial dose), and early discontinuation ( $<4$ cycles). Descriptive analyses were conducted per hospital, year of diagnosis and several patient characteristics. Predictors for early discontinuation were explored in a logistic regression model.

Results: Overall, $47.9 \%$ of 2158 patients that were included received systemic treatment and $33.7 \%$ of those received second-line treatment. Treatment frequencies were different between age categories, disease stage, PS and hospital $(p<0.001)$. Half of the patients received $<4$ cycles and dose reductions were found for $20 \%$ of all patients. Interhospital differences were observed for early discontinuation and the number of switches. PS2-3 was associated with early discontinuation (OR $1.97(p=0.007)$. Median survival was not different between hospitals and years of diagnosis.

Discussion: We provided detailed overview of day-to-day systemic treatment of NSCLC for six hospitals that (a) can fuel interhospital discussion to streamline treatment towards best practice and (b) can serve as reference data for follow-up of the adoption of novel systemic treatment options for advanced lung cancer. 


\section{INTRODUCTION}

Non-small cell lung cancer (NSCLC) represents 85 percent of all lung cancer diagnoses in The Netherlands [1]. The majority of patients present with locally advanced or metastatic disease at the time of diagnosis [2]. The 1-year survival rate of metastatic non-small cell lung cancer (NSCLC) is only 20\% [1]. Palliative treatments that are provided to these patients aim to preserve or improve quality of life, lengthen life or decrease disease burden. Palliation can consist of symptom relief (best supportive care) or systemic treatment targeted at tumour tissue.

There are various drugs available to target tumour tissue including systemic chemotherapy, targeted therapy - tyrosine kinase inhibitors (TKIs) targeting specific genetic phenotypes - and immunotherapy [3]. Although many (inter)national guidelines are available to support treatment decision-making, little is known about how these guidelines resonance in day-to-day clinical practice in terms of utilization and clinical outcomes. Also, day-to-day clinical practice may well vary from hospital to hospital.

In the context of a Value Based Health Care system, the present study aims to give a detailed overview of day-to-day practice in the systemic treatment of NSCLC stage IIIB/IV and its clinical outcomes in six large teaching hospitals in the Netherlands over a period of five years (2008-2012). Clinical outcomes studied include overall survival, dose-reductions, treatment switches and early treatment discontinuation.

\section{MATERIALS AND METHODS}

Clinical data were collected from six large teaching hospitals in The Netherlands. These six hospitals work together under the name of Santeon, a Dutch nationwide Hospital Group. Santeon serves more than $12.5 \%$ of the Dutch lung cancer patient population. The individual hospitals are: Canisius Wilhelmina Hospital (Nijmegen), Catharina Hospital (Eindhoven), Martini Hospital (Groningen), Medisch Spectrum Twente (Enschede), Onze Lieve Vrouwe Gasthuis (Amsterdam) and St. Antonius Hospital (Nieuwegein/Utrecht). In 2012, Santeon established the Care for Outcome (CfO) registry that includes outcome data from all patients diagnosed with lung and prostate cancer in one of the six hospitals from 2008 onwards. The CfO registry is built on the general Dutch Cancer Registry (managed by the Netherlands Comprehensive Cancer Organisation (IKNL)) and subsequently enriched with clinical data from the hospitals (through extensive medical chart review and norminalisation of data). These clinical data include, amongst others, tumour characteristics, patient comorbidities, ECOG performance status, treatment planning and clinical outcomes. Trained medical students supervised by a pulmonary physician extracted all items. Parallel to the $\mathrm{CfO}$ registry Santeon established the Santeon Farmadatabase that holds all prescribed and dispensed drugs at the individual patient level for all patients receiving care in one of 
the six hospitals from 2010 onwards. For every prescription the database includes, amongst others, drug name, dosage, date of administration, and administration route. The study has been approved by the local research ethics committee of each individual hospital.

\section{Patient selection and data collection}

From the CfO database we extracted data from all patients that (1) were diagnosed with NSCLC in one of the Santeon hospitals, (2) had clinical stage IIIB or IV (including both patients with and without systemic treatment), and (3) had follow-up data (vitality status) available. Patients were ignored if the hospital of primary treatment was not the hospital of diagnosis. For this selection of patients, we extracted all prescribed and dispensed drug data from individual hospital pharmacy information systems. Because in the time period under study the tyrosine-kinase inhibitor could also be dispensed from communitypharmacies we additionally reviewed all medical charts if one or more prescription were identified in the hospital pharmacy system to gather data about initiation data and duration of treatment.

\section{Study parameters}

From the drug prescription and dispensing data we constructed an overview of systemic treatments for every patient including the type of systemic treatment and whether it was first, second, or further line of treatment. If the start of a subsequent regimen was less than 90 days after the theoretical end date of the first-line treatment (based on 3 weeks per cycle), medical charts were reviewed to determine whether the change of treatment was due to ineffectiveness (progression, no regression) or toxicity. Switch to another treatment option more than 90 days after the theoretical end date of the first-line treatment and switch to another treatment option due to ineffectiveness was considered next line treatment. Early switch to another treatment due to toxicity was considered the same line of treatment. Additional cycles after a switch due to toxicity were added to the initially administered number of cycles.

\section{Outcome parameters}

Individual patient survival times were calculated as the time difference (in days) between date of diagnosis and date of death (vitality status as known on 31-12-2014). As a proxy for toxicity, based on the drug prescription and dispensing data, we constructed early discontinuation of treatment ( $<4$ cycles) and dose reductions ( $<80 \%$ of the initial dose) as additional patients' outcomes.

\section{Statistical analysis}

SPSS Statistics (version 24.0) was used for the statistical analysis. Age was categorized into three categories: under 65 years, 65-74, and 75 years or older. Tumour histology was categorized into squamous and non-squamous. Descriptive data were composed for the total sample, 
per year and per hospital stratified by various patient characteristics. From the individual treatment data several indicators were constructed including: percentage of patients treated with chemotherapy, percentage of different first-line treatments options, and number and percentage of switches, dose reductions, and early discontinuation.

Between groups frequencies were compared using a chi square test, means using a one-way ANOVA test, and survival time between categories using a Log-Rank test. A p-value of $<0.05$ was considered significant. To identify patient characteristics that are associated with early discontinuation without any subsequent systemic treatment, univariate and multivariable logistic regression was planned. Patients receiving a second-line treatment were excluded from the analysis.

\section{RESULTS}

A total of 2619 NSCLC stage IIIB/IV patients could be selected from the CfO lung cancer database. From this selection, we excluded 13 patients because follow-up data was not available and 448 patients because the hospital of primary treatment was not the hospital of diagnosis resulting in a cohort of 2158 patients. The patient characteristics are described in Table 1. The majority of patients ( $81.8 \%$ ) were diagnosed with stage IV NSCLC and the mean age was 68 years. More than $25 \%$ of patients had a PS of 2 or 3 at the time of diagnosis. No statistical differences were observed between hospitals for disease stage, gender, and age whereas PS did significantly differ between hospitals $(p<0.05)$.

Overall, $47.9 \%$ of all patients received any systemic treatment, $33.7 \%$ of those receiving first-line treatment received second-line treatment. Third-line was started in $30.5 \%$ of those receiving second-line treatment. A small but increasing number of patients was treated with maintenance treatment as part of their first-line treatment $(0.5 \%$ for year of diagnosis 2008, 5.8\% in 2012) but no increase in the use of systemic treatment was observed over the years for any of the lines of treatment (Supplemental Table 1). Fig. 1 depicts the percentage of patients treated with systemic treatment stratified by age category, gender, disease stage, PS and hospital of diagnosis and treatment. Treatment frequencies were statistically different between age categories, disease stage, PS and hospital (all $p<0.001$ ). Patients not receiving systemic treatment received best supportive care (including palliative radiotherapy in $12.7 \%$ of those patients).

Variation in first-line treatments was limited with seven regimens accounting for almost $90 \%$ of all first-line treatments. Moreover more than $75 \%$ of all first-line regimens were platinum based regimens with either gemcitabine or pemetrexed. The top-7 prescribed first-line regimens and their frequencies (\%) are presented in Fig. 2. A quarter of the other group was represented by TKI users.

Once first-line treatment was started, approximately half of the patients received four or more cycles (Table 2). Of note, additional cycles after discontinuation of the initial 


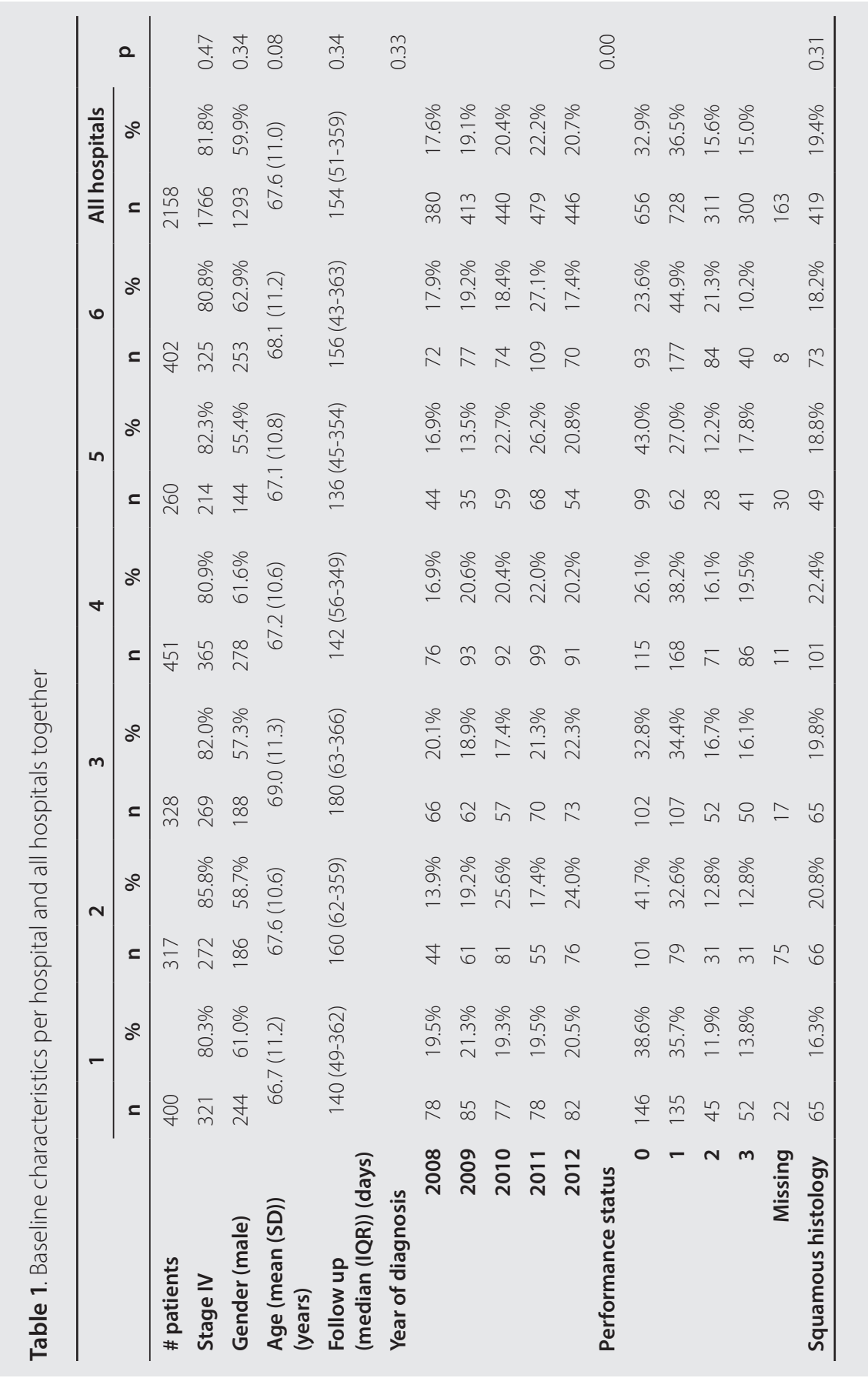




\section{Systemic treatment (\%)}

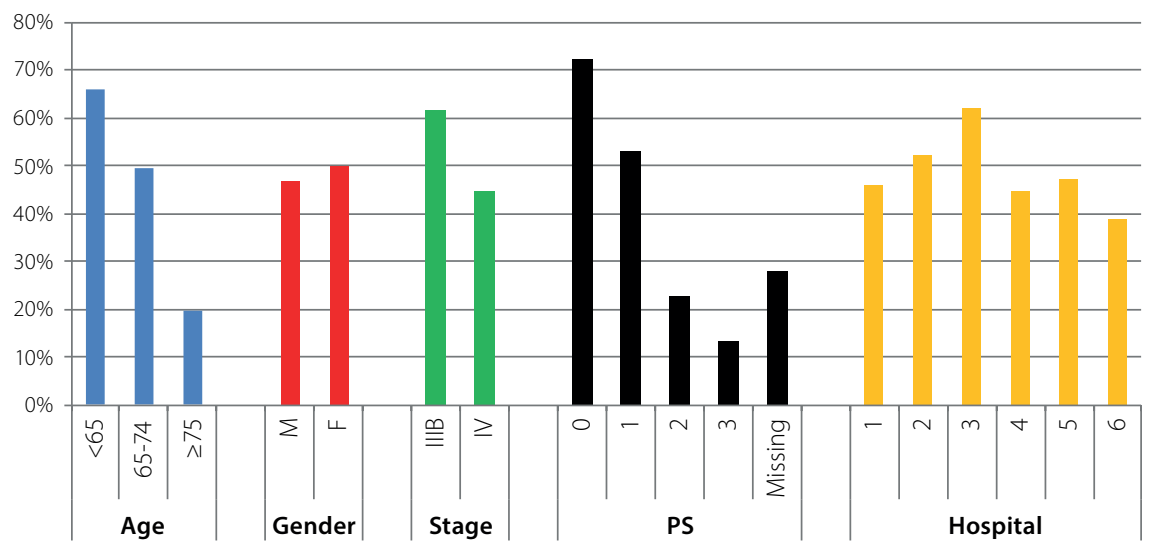

Fig. 1. Histogram. Percentage of patients systemically treated stratified by age, gender, disease stage, PS and hospital.

treatment due to toxicity were considered cycles part of first-line treatment (e.g. 1 cycle of cisplatin gemcitabine followed by 3 cycles of carboplatin gemcitabine results in a total of 4 cycles). Furthermore, dose reductions were found for $20 \%$ of all patients in any line of treatment (Table 2). A switch to other systemic agents due to toxicity was found in 15\% of all first-line treatment, mostly involving cisplatin based regimens. Significant interhospital differences were observed for the number of cycles administered ( $<4$ cycles versus $>=4$ cycles) and the number of switches (Table 2).

Univariate logistic regression only revealed performance status to be associated with the outcome of early discontinuation in 686 patients receiving first-line treatment only. Performance status 0-1 and 2-3 were grouped because odds ratios (OR) were similar (PSO, reference; PS1, 1.17; PS2, 2.19; PS3, 2.00). The OR for PS 2-3 versus PS 0-1 for early discontinuation was $1.97(1.20-3.23, p=0.007)$.

Median survival times in days for the total population, untreated and systemically treated population only are presented in Table 3. In the total population, the median survival was significantly higher for disease stage IIIB, women, younger patients and better PS. There was no difference in median survival between hospitals and between years of diagnosis. Although the median survival did statistically differ between age categories for the total population and the treated population, the median survival was not lower for patients of 75 years of age and older when treated systemically. 


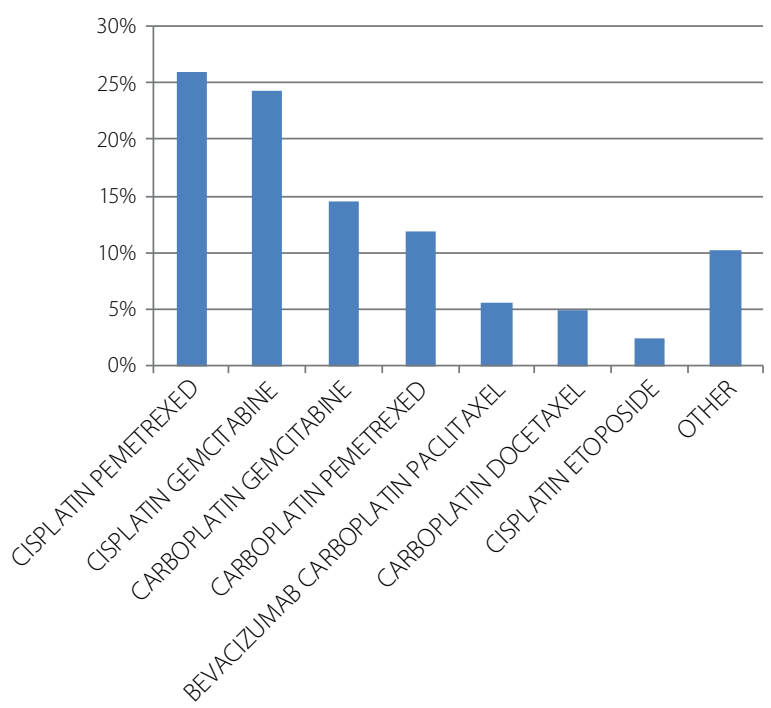

Fig. 2. Regimens started in the first-line of treatment presented as percentage.

Table 2. Frequencies and percentages of dose reductions, cycles, and switches per hospital and for all hospitals.

\begin{tabular}{|c|c|c|c|c|c|c|c|c|c|c|c|c|c|c|c|}
\hline & & 1 & & 2 & & 3 & & 4 & & 5 & & 6 & \multicolumn{2}{|c|}{$\begin{array}{c}\text { all } \\
\text { hospitals }\end{array}$} & $p$ value \\
\hline \multicolumn{16}{|c|}{ Number of patients } \\
\hline & & 184 & & 66 & & 03 & & 202 & & 123 & & 156 & 10 & 34 & \\
\hline \multicolumn{16}{|c|}{ Patients with dose reduction ( 1 or all drugs within any line) } \\
\hline & 37 & $20 \%$ & 28 & $17 \%$ & 48 & $24 \%$ & 48 & $24 \%$ & 28 & $23 \%$ & 19 & $12 \%$ & 208 & $20 \%$ & 0.052 \\
\hline \multicolumn{16}{|c|}{ Number of cycles } \\
\hline$>=4$ & 92 & $50 \%$ & 121 & $73 \%$ & 111 & $55 \%$ & 80 & $40 \%$ & 48 & $39 \%$ & 81 & $52 \%$ & 533 & $52 \%$ & $<0.001$ \\
\hline 3 & 41 & $22 \%$ & 14 & $8 \%$ & 34 & $17 \%$ & 44 & $22 \%$ & 20 & $16 \%$ & 27 & $17 \%$ & 180 & $17 \%$ & \\
\hline 2 & 33 & $18 \%$ & 16 & $10 \%$ & 33 & $16 \%$ & 50 & $25 \%$ & 24 & $20 \%$ & 22 & $14 \%$ & 178 & $17 \%$ & \\
\hline 1 & 18 & $10 \%$ & 15 & $9 \%$ & 25 & $12 \%$ & 28 & $14 \%$ & 31 & $25 \%$ & 26 & $17 \%$ & 143 & $14 \%$ & \\
\hline \multicolumn{16}{|c|}{ Number of switches } \\
\hline & 41 & $22 \%$ & 37 & $22 \%$ & 21 & $10 \%$ & 27 & $13 \%$ & 7 & $6 \%$ & 23 & $15 \%$ & 156 & $15 \%$ & $<0.001$ \\
\hline
\end{tabular}




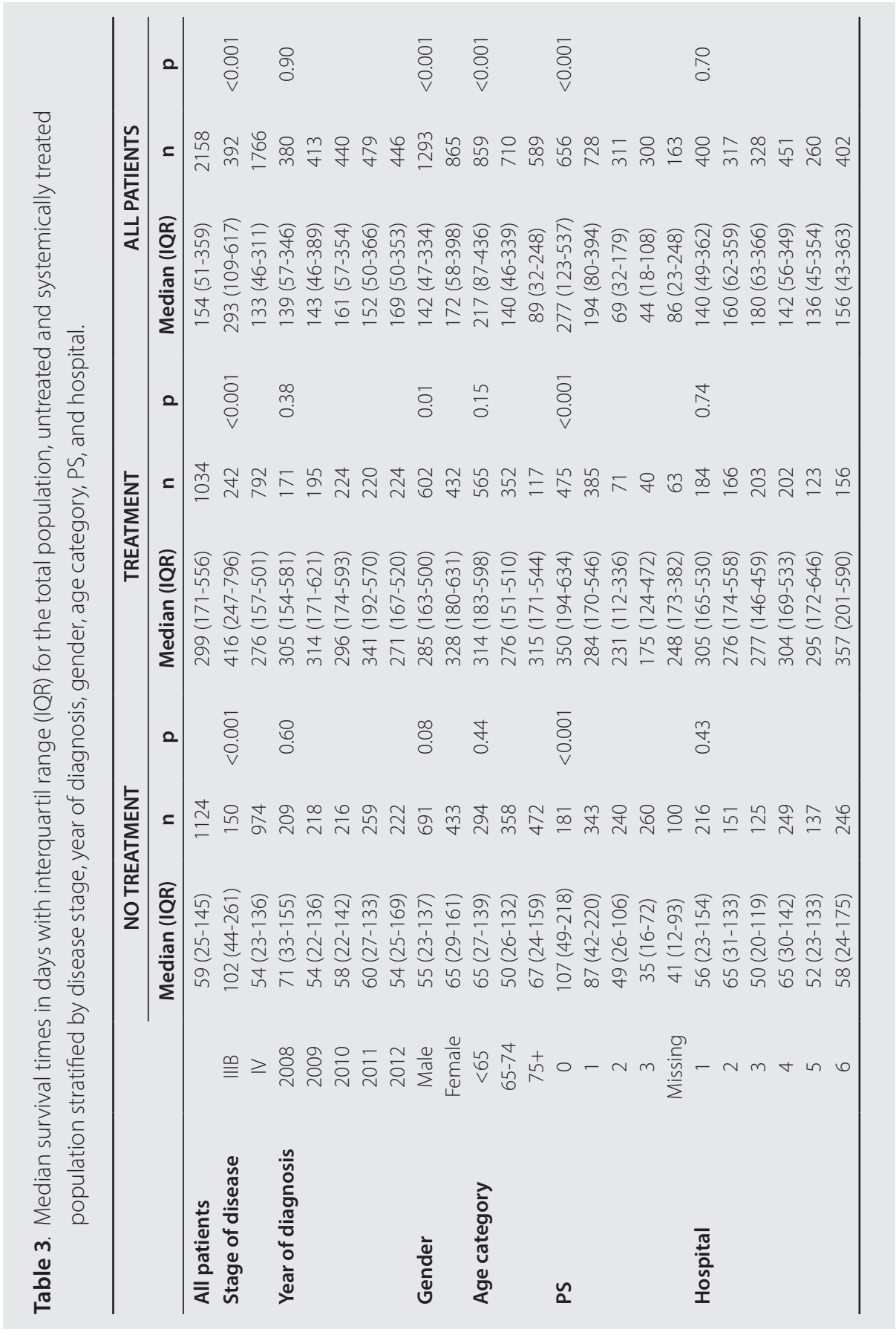




\section{DISCUSSION}

We have provided a detailed overview of daily practice in the systemic palliative treatment of NSCLC stage IIIB/IV in six Dutch teaching hospitals over a period of five years (2008-2012). Additionally, we investigated possible associations between patient characteristics and early discontinuation and we evaluated overall survival.

In this large cohort of NSCLC stage IIIB/IV $47.9 \%$ of patients received first-line systemic treatment (44.8\% stage IV, 61.7\% stage IIIB). In line with our results, Brule et al. recently showed in their Canadian single centre study 55\% of all advanced NSCLC patients received palliative systemic treatment in the same time window (2009-2012) [4]. Another study found $34 \%$ of all patients diagnosed with stage IV NSCLC and seen by an oncologist from 2005 to 2009 received systemic treatment [5]. Second-line treatment was started for $51 \%$ [4] and $33 \%$ [5] of all patients treated with first-line systemic treatment, whereas 34\% of the patients in our study received second-line treatment.

The median survival of patients that were treated systemically was 10.0 months, comparable to previously reported real-world survival outcomes $[4,5,12]$. Consistently with other research, we found patients $>75$ years to have a median survival similar to those of younger patients [5]. Furthermore, the survival of systemically treated patients with a PS of 3 was 5.8 months whereas Salloum et al. showed in their observational (time window 2000-2007) study patients stage IIIB-IV with a poor PS (3 or 4) to have a median survival of 4.8 months [6]. Furthermore, we did not observe an increase in overall survival over the years. A large observational study from the United States also found no difference in overall survival for patients diagnosed 2011 as compared to 2007 reporting a median survival of respectively 9.2 and 9.3 months [12].

To our knowledge no other observational study has investigated the number of cycles and dose reductions as outcome parameters in routine care. We showed $49 \%$ of patients received less than four cycles of their first-line treatments. Comparing our results to the number of cycles administered in clinical trials is challenging given that many trials report median number of cycles and give up to six cycles [7-9] whereas in Dutch clinical practice this is mostly four. Nevertheless, our logistic regression analysis shows an association between early discontinuation and poor PS, a population generally underrepresented in randomized clinical trials. Groene et al. conducted a similar analysis in patients diagnosed with invasive epithelial cancer of the oesophagus or stomach [10]. In addition to a poor PS, they found age to be inversely associated with palliative treatment completion, concluding that this information should be considered when balancing treatment benefits, toxicity and patients quality of life.

Interhospital differences were found for several outcomes such as (a) the proportion of patients treated systemically and (b) the number of cycles and switches. These observations show that, although guidelines exist, translation to clinical practice can result in significant interhospital variation in outcomes presented in our study. This variation should be further 
investigated but could reflect differences of insight regarding the systemic treatment of elderly, patients with poor PS, and handling of toxicity. Our data can fuel interhospital discussion with the aim of streamlining NSCLC treatment towards best practice. In terms of survival, interhospital differences for the proportion of patients treated systemically did not translate into survival differences considering the group of patients as a whole (treated and untreated). This observation and the fact that systemic treatment is strongly associated with a survival benefit, could reflect interhospital patient selection for systemic treatment. Some doctors will treat only those patient that are surely fit for systemic treatment whereas others will also start treatment for patients who are more likely to suffer from the toxic effects of systemic treatment. The net survival result for both approaches may be the same, whereas other outcomes that relate to the quality of life may well differ. More research is needed to address this hypothesis.

Strengths of our study include (a) large cohort representing an unselected population from patients diagnosed with advanced lung cancer in the Netherlands, (b) highly detailed and validated data about applied pharmacotherapy (c) the availability of a large set of relevant patient/clinical characteristics with low numbers of missing data (d) and the possibility to analyse dose reductions, switches and number of cycles administered. A limitation of our study is the incompleteness of data about TKI use. Until the end of 2012, Dutch patients were allowed to collect these medications through community pharmacies, whereas they are dispensed by the hospital pharmacy since. We think, however, that this limitation does not affect our analyses regarding first-line treatment as these drugs are only applied as second or later line of treatment in the study period.

In this study, we have presented a detailed overview of daily practice in the systemic palliative treatment of NSCLC stage IIIB/IV over the years 2008-2012. Such detailed data can serve as reference data for follow-up of the adoption of novel systemic treatment options for advanced lung cancer. Besides this, the constructed database provides a valuable non-trial context to study real-world effectiveness of both existing systemic treatments and future treatments. Comparative effectiveness research using real-world data can help answer questions that relate to the external validity of results from RCTs [11]. Moreover, the rapid development of new medical treatments will give rise to many more clinical questions that need to be answered but are financially and practically impossible to answer with $\mathrm{RCTs}$ only. As for $\mathrm{CfO}$, patient reported outcome measures (PROMs) are prospectively collected as part of the Santeon CfO program since 2014, allowing evaluation of drug effectiveness in NSCLC beyond the outcome overall survival in the near future. 
50 | CHAPTER 2

\section{ACKNOWLEDGEMENTS}

The authors thank Dr. Wim van den Bosch for establishing the Care for Outcome registry. Dr. Hadewig Colen, Dr. Mirjam Crul, Dr. Maarten Deenen, Dr. Rene van Hulst, and Mr. Yuhan Kho are acknowledged for their efforts to complete and validate the hospital pharmacy data. 


\section{REFERENCES}

1. Nederlandse Kankerregistratie Available at: http://www.cijfersoverkanker.n//home-27.html. Accessed 9/25/2014, 2014.

2. Molina JR, Yang P, Cassivi SD, et al. Non-small cell lung cancer: epidemiology, risk factors, treatment, and survivorship. Mayo Clin Proc 2008;83: 584-594.

3. Reck M, Popat S, Reinmuth $\mathrm{N}$, et al. Metastatic non-small-cell lung cancer (NSCLC): ESMO Clinical Practice Guidelines for diagnosis, treatment and follow-up. Ann Oncol 2014;25 Suppl 3: iii27-39.

4. Brule SY, Al-Baimani $\mathrm{K}$, Jonker $\mathrm{H}$, et al. Palliative systemic therapy for advanced non-small cell lung cancer: Investigating disparities between patients who are treated versus those who are not. Lung Cancer 2016;97: 15-21.

5. Sacher AG, Le LW, Lau A, et al. Real-world chemotherapy treatment patterns in metastatic non-small cell lung cancer: Are patients undertreated? Cancer 2015;121: 2562-2569.

6. Salloum RG, Smith TJ, Jensen GA, et al. Survival among non-small cell lung cancer patients with poor performance status after first line chemotherapy. Lung Cancer 2012;77: 545-549.

7. Scagliotti GV, Parikh P, von Pawel J, et al. Phase III study comparing cisplatin plus gemcitabine with cisplatin plus pemetrexed in chemotherapy-naive patients with advanced-stage non-small-cell lung cancer. J Clin Oncol 2008;26: 3543-3551.

8. Scagliotti GV, De Marinis F, Rinaldi M, et al. Phase III randomized trial comparing three platinum-based doublets in advanced non-small-cell lung cancer. J Clin Oncol 2002;20: 4285-4291.

9. Paz-Ares L, Mezger J, Ciuleanu TE, et al. Necitumumab plus pemetrexed and cisplatin as first-line therapy in patients with stage IV non-squamous non-small-cell lung cancer (INSPIRE): an open-label, randomised, controlled phase 3 study. Lancet Oncol 2015;16: 328-337.

10. Groene $\mathrm{O}$, Crosby T, Hardwick RH, et al. A population-based observational study on the factors associated with the completion of palliative chemotherapy among patients with oesophagogastric cancer. BMJ Open 2015;5: e006724-2014-006724.

11. Lyman GH, Levine M. Comparative effectiveness research in oncology: an overview. J Clin Oncol 2012;30: 4181-4184.

12. Bradley C, Yabroff KR, Mariotto AB, et al. Antineoplastic Treatment of Advanced-Stage Non-Small-Cell Lung Cancer: Treatment, Survival, and Spending (2000 to 2011). J Clin Oncol. 2017; Jan 3: JCO2016694166. 


\section{APPENDIX CHAPTER 2}

\section{Supplemental Table 1}

\begin{tabular}{|c|c|c|c|c|c|c|c|c|c|c|c|c|c|c|}
\hline \multirow[b]{2}{*}{$\begin{array}{l}\text { Year of } \\
\text { diagnosis }\end{array}$} & \multicolumn{2}{|c|}{1} & \multicolumn{2}{|c|}{2} & \multicolumn{2}{|c|}{3} & \multicolumn{2}{|r|}{4} & \multicolumn{2}{|r|}{5} & \multicolumn{2}{|r|}{6} & \multicolumn{2}{|c|}{$\begin{array}{c}\text { All } \\
\text { hospitals }\end{array}$} \\
\hline & $n$ & $\%$ & $\mathbf{n}$ & $\%$ & $\mathrm{n}$ & $\%$ & $n$ & $\%$ & $\mathrm{n}$ & $\%$ & $n$ & $\%$ & n & $\%$ \\
\hline \multicolumn{15}{|l|}{ 1st line } \\
\hline 2008-2012 & 184 & $46.0 \%$ & 166 & $52.4 \%$ & 203 & $61.9 \%$ & 202 & $44.8 \%$ & 123 & $47.3 \%$ & 156 & $38.8 \%$ & 1034 & $47.9 \%$ \\
\hline 2008 & 35 & $44.9 \%$ & 26 & $59.1 \%$ & 40 & $60.6 \%$ & 29 & $38.2 \%$ & 15 & $34.1 \%$ & 26 & $36.1 \%$ & 171 & $45.0 \%$ \\
\hline 2009 & 42 & $49.4 \%$ & 29 & $47.5 \%$ & 41 & $66.1 \%$ & 47 & $50.5 \%$ & 14 & $40.0 \%$ & 22 & $28.6 \%$ & 195 & $47.2 \%$ \\
\hline 2010 & 43 & $55.8 \%$ & 42 & $51.9 \%$ & 37 & $64.9 \%$ & 43 & $46.7 \%$ & 27 & $45.8 \%$ & 32 & $43.2 \%$ & 224 & $50.9 \%$ \\
\hline 2011 & 31 & $39.7 \%$ & 29 & $52.7 \%$ & 43 & $61.4 \%$ & 39 & $39.4 \%$ & 34 & $50.0 \%$ & 44 & $40.4 \%$ & 220 & $45.9 \%$ \\
\hline 2012 & 33 & $40.2 \%$ & 40 & $52.6 \%$ & 42 & $57.5 \%$ & 44 & $48.4 \%$ & 33 & $61.1 \%$ & 32 & $45.7 \%$ & 224 & $50.2 \%$ \\
\hline
\end{tabular}

\section{1 ste line maintenance}

$\begin{array}{rllllllllllllll}2008-2012 & 9 & 2.3 \% & 8 & 2.5 \% & 10 & 3.0 \% & 5 & 1.1 \% & 4 & 1.5 \% & 32 & 8.0 \% & 68 & 3.2 \% \\ 2008 & 1 & 1.3 \% & 0 & 0.0 \% & 0 & 0.0 \% & 0 & 0.0 \% & 0 & 0.0 \% & 1 & 1.4 \% & 2 & 0.5 \% \\ 2009 & 2 & 2.4 \% & 0 & 0.0 \% & 2 & 3.2 \% & 2 & 2.2 \% & 0 & 0.0 \% & 3 & 3.9 \% & 9 & 2.2 \% \\ 2010 & 2 & 2.6 \% & 0 & 0.0 \% & 1 & 1.8 \% & 0 & 0.0 \% & 0 & 0.0 \% & 3 & 4.1 \% & 6 & 1.4 \% \\ 2011 & 4 & 5.1 \% & 5 & 9.1 \% & 2 & 2.9 \% & 1 & 1.0 \% & 1 & 1.5 \% & 12 & 11.0 \% & 25 & 5.2 \% \\ 2012 & 0 & 0.0 \% & 3 & 3.9 \% & 5 & 6.8 \% & 2 & 2.2 \% & 3 & 5.6 \% & 13 & 18.6 \% & 26 & 5.8 \%\end{array}$

\section{2nd line}

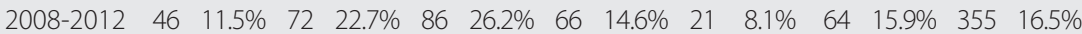

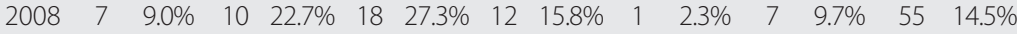

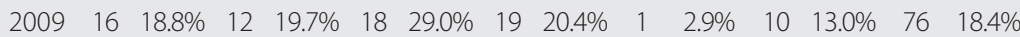

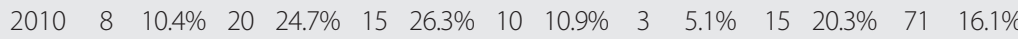

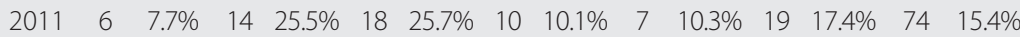

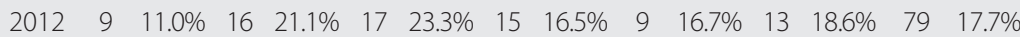

\section{3rd line}

\begin{tabular}{rcccccccccccccc}
$2008-2012$ & 12 & $3.0 \%$ & 20 & $6.3 \%$ & 25 & $7.6 \%$ & 23 & $5.1 \%$ & 5 & $1.9 \%$ & 23 & $5.7 \%$ & 108 & $5.0 \%$ \\
2008 & 2 & $2.6 \%$ & 3 & $6.8 \%$ & 5 & $7.6 \%$ & 3 & $3.9 \%$ & 0 & $0.0 \%$ & 3 & $4.2 \%$ & 16 & $4.2 \%$ \\
2009 & 6 & $7.1 \%$ & 4 & $6.6 \%$ & 4 & $6.5 \%$ & 9 & $9.7 \%$ & 1 & $2.9 \%$ & 6 & $7.8 \%$ & 30 & $7.3 \%$ \\
2010 & 0 & $0.0 \%$ & 6 & $7.4 \%$ & 4 & $7.0 \%$ & 5 & $5.4 \%$ & 2 & $3.4 \%$ & 3 & $4.1 \%$ & 20 & $4.5 \%$ \\
2011 & 1 & $1.3 \%$ & 4 & $7.3 \%$ & 9 & $12.9 \%$ & 3 & $3.0 \%$ & 1 & $1.5 \%$ & 5 & $4.6 \%$ & 23 & $4.8 \%$ \\
2012 & 3 & $3.7 \%$ & 3 & $3.9 \%$ & 3 & $4.1 \%$ & 3 & $3.3 \%$ & 1 & $1.9 \%$ & 6 & $8.6 \%$ & 19 & $4.3 \%$ \\
\hline
\end{tabular}


SYSTEMIC TREATMENT AND OS FOR NSCLC STAGE IIIB/IV 2008-2012 | 53 



\title{
Chapter 3
}

Systematic evaluation of the efficacyeffectiveness gap of systemic treatments in metastatic non-small cell lung cancer

\author{
Christine M. Cramer-van der Welle, Bas J.M. Peters, Franz M.N.H. Schramel, Olaf H. Klungel,
} Harry J.M. Groen, Ewoudt M.W. van de Garde, for the Santeon NSCLC Study Group 


\section{ABSTRACT}

The divergence between clinical trial results and real-world outcomes is largely unknown for many cancer types. The present study aims to overall assess the efficacy-effectiveness gap (difference between outcomes in clinical trials and the real-world) in systemic treatment for metastatic non-small cell lung cancer (NSCLC).

All patients diagnosed with stage IV NSCLC between 2008 and 2014 within a network of seven Dutch large teaching hospitals (Santeon) were studied. For every patient, an efficacy-effectiveness factor (EE-factor) was calculated by dividing individual patients' overall survival (OS) by the pooled median OS assessed from clinical trials with the respective treatment.

From 2989 diagnosed patients, 1214 (41\%) started with first-line treatment. For all studied regimens, real-world OS was shorter than OS reported in clinical trials. Overall, the EE-factor was 0.77 (95\% Cl 0.70-0.85; $\mathrm{p}<0.001)$. Real-world patients completed their treatment plan less often and proceeded less frequently to further lines of treatment. These parameters together with Eastern Cooperative Oncology Group performance status explained 35\% of the variation in EE-factor.

Survival of patients with metastatic NSCLC treated with chemotherapy or targeted therapy in real-world practice is nearly one quarter shorter than for patients included in trials. Patients' performance status, earlier discontinuation, and fewer subsequent lines of treatment partly explained this difference. 


\section{INTRODUCTION}

Non-small cell lung cancer (NSCLC) represents 77\% of all lung cancer diagnoses in the Netherlands [1]. The majority of patients present with locally advanced or metastatic disease at the time of diagnosis [2, 3]. The overall 1-year survival rate of metastatic NSCLC (stage IV) is only $22 \%$ [1]. Palliative treatment provided to these patients can consist of symptomatic relief by best supportive care or systemic treatment targeted at tumour tissue.

Numerous phase 3 clinical trials have shown the superiority of systemic treatment over best supportive care in patients with metastatic NSCLC $[2,4]$. However, data from oncology clinical trials, although providing critical evidence of clinical activity, do not provide adequate information to determine the impact of treatments when used in the real-world setting. Due to strict patient inclusion criteria, important patient characteristics predictive for treatment response are often underrepresented in clinical trials. This results in uncertainty about how results from clinical trials translate to the real-world population. However, this information is crucial for both patients and physicians to decide on what type of treatment to choose, if any, especially in the palliative setting, where quality of life may outweigh the possible extension of survival by systemic therapy.

Some efforts have been made to provide insight into treatment outcomes in real-world populations with lung cancer versus clinical trials. For example, Zhu et al. [5] showed a shorter median survival estimate in routine practice compared to participants in the Eastern Cooperative Oncology Group (ECOG) 4599 trial, and Jungels et al. [6] showed comparable response rates but shorter survival in the real-world for pemetrexed in patients previously treated with chemotherapy. Another example is a Canadian study which showed that second-line treatment with erlotinib resulted also in a shorter median survival in clinical practice versus in a clinical trial setting [7]. Outside the few single treatment comparisons, a comprehensive overview of the possible divergence between clinical trials and routine care settings is still lacking for lung cancer [8].

Considering that a significant proportion of metastatic NSCLC patients are treated outside clinical trials, the present study aims to overall explore the effectiveness of systemic treatment in real-world practice versus efficacy data from clinical trials (efficacy-effectiveness (EE) gap), and to look into the factors that may explain a gap.

\section{METHODS}

\section{Data source}

This study was conducted with clinical data originating from a network of seven large (nonuniversity) teaching hospitals geographically spread in the Netherlands, named Santeon (Appendix 4). Santeon was established in 2007 and serves $>12 \%$ of the Dutch patient population 
In 2012, Santeon constructed the Care for Outcome registry, which includes clinical and outcome data from all patients diagnosed with lung cancer in one of the hospitals from 2008 onwards. These data include tumour characteristics, patient characteristics, treatment planning, and clinical outcomes. Information on how data is procured, standardized, and validated can be found elsewhere [9].

Parallel to the Care for Outcome registry, Santeon established the Santeon Farmadatabase, which comprises all prescribed and dispensed drugs at the individual patient level for all patients receiving care in one of the hospitals from 2010 onwards. For every prescription the database includes, among others, drug name, dosage, date of administration and administration route. Full details of the Santeon Farmadatabase are described elsewhere [10]. This study was discussed by a medical research ethics committee and the need for informed consent was waived because the study was considered exempt from review.

\section{Study participants}

Within the Care for Outcome registry, all patients with metastatic (stage IV) NSCLC diagnosed between 2008 and 2014 were selected for this study (staging in 2008-2009 was based on the sixth edition of the American Joint Committee on Cancer TNM (turmour, node, metastasis) staging system for lung cancer and from 2010 onwards was based on the seventh edition; in both editions, the same criteria for stage IV NSCLC were used). Patients with vital status "emigrated" (at January 31, 2017) were excluded, due to their unknown survival status. For the remaining patients, the following clinical patient characteristics were collected: age at diagnosis, sex, Charlson comorbidity index (CCI) and separate comorbidities (e.g. diabetes), ECOG performance status (PS), histology, and year of diagnosis.

Next, for every patient we searched the Farmadatabase to determine whether or not they received systemic treatment for metastatic NSCLC. For the years 2008 and 2009, individual pharmacy systems were checked for missing data about systemic treatment.

\section{Identification of systemic treatment per patient}

For every patient, an overview of systemic treatments was constructed from the recorded prescription data, including the identification of regimens and whether it was first, second or further line of treatment. The initial systemic treatment following the date of diagnosis was defined as first-line treatment. Switching to another regimen $>90$ days after the theoretical end date of the previous regimen was (pragmatically) considered as a subsequent line of treatment. Switches to another regimen $<90$ days after the theoretical end date of the previous regimen were reviewed manually to determine a switch due to disease progression (subsequent line) or toxicity (same line of treatment).

Based on all identified regimens, a list was constructed that covered $>90 \%$ of all first-line treatment regimens. For practical reasons, the remaining (rarely applied) regimens were put into an "other" category. 


\section{Systematic literature review for reference outcomes}

A systematic literature search and meta-analysis were conducted to obtain a pooled efficacy result for the first-line treatment regimens from the aforementioned list. First, an overview of all corresponding phase III studies was constructed from an extensive search in the PubMed, Embase, and CENTRAL (Cochrane library) databases (searched up to September 1, 2017). The exact details of this search are provided in Appendix 1. An article was considered eligible if all the following criteria were met. 1) Patients diagnosed with NSCLC; 2) main article of a phase III randomised trial; 3) intervention under study is one of the regimens identified in our data; 4) patients with stage IV disease (possibly in combination with stage IIIB); and 5) overall survival (OS) as outcome. To create a homogenous selection of articles, we excluded articles about a secondary analysis (e.g. subgroup analysis, post hoc analysis, or intermediate analysis); articles with randomisation between variations of the same systemic treatment (e.g. differences in timing, dose or duration); articles about systemic treatment together with radiotherapy; articles with randomisation after progression of disease; and articles that did neither provide confidence intervals for the OS nor a survival curve.

The first selection of articles was screened for eligibility based on title and abstract by two reviewers (CC and EvdG or FS or BP). Consensus was sought in case of differences between reviewers. Subsequently, full-text articles were examined by a single reviewer (CC), with a second reviewer in case of uncertainty about eligibility (EvdG). No reference tracking was performed. Appendix 2 provides in more detail per regimen the yield of the systematic review and the meta-analysis data. From all included articles, the outcome (OS in months), inclusion period, inclusion criteria, line of treatment, patient characteristics, percentage of patients with a completed treatment plan and percentage of patients who received a subsequent line of systemic treatment was extracted. In case the median OS was not described, this outcome was derived from the survival curve.

The computer programme Review Manager (Version 5.3; The Nordic Cochrane Centre, The Cochrane Collaboration, Copenhagen, Denmark; 2014) was used to combine all reported OS outcomes from the clinical trials to estimate the "reference outcome" (pooled OS) per regimen from all included articles. Fixed-effect or random-effect (DerSimonian and Laird) measures were calculated depending on the level of heterogeneity $(p<0.05$ level). The required standard error has been calculated using the equation $\mathrm{SE}=$ (upper limit 95\% Cl - lower limit 95\% Cl)/2x1.96, or using the interquartile range (IQR) derived from the Kaplan-Meier curve, where $\mathrm{SD} \approx(\mathrm{QQR}) / 1.35$ and $\mathrm{SE}=\mathrm{SD} / \sqrt{ } \mathrm{N}$.

\section{Real-world treatment outcomes}

For every patient, OS was calculated based on the time between start date of systemic treatment and date of death. Patients still alive at January 31, 2017 were given this end of follow-up date as imputed date of death $(n=54)$. Subsequently, we calculated an EE factor for every patient by dividing the individual real-world OS by the pooled clinical trial OS for 
the first-line treatment regimen. This EE factor should be interpreted as how an individual outcome relates to the median OS from the corresponding clinical trial (e.g. an EE factor of 0.80 means that survival is $20 \%$ shorter). Besides survival, toxicity was assessed by using percentage of treatment switches, dose reductions ( $\leq 80 \%$ of the initial dose) and early discontinuation (fewer than four cycles or tyrosine kinase inhibitor use $<1$ month) as proxy.

\section{Statistical analysis}

Statistical software (SPSS version 24 for Windows; IBM, Armonk, NY, USA) was used for statistical analysis. Continuous data were expressed as mean \pm SD or median (range) when appropriate. Categorical data were analysed using Chi-square and continuous data using t-tests, rank tests and one-way ANOVA when appropriate. First, to assess the presence of an EE gap, the distribution of the calculated EE factors was tested relative to 1.0 by using the Wilcoxon signed-rank test. If that distribution does not have a median of 1.0, the null hypothesis (that the median OS in the real-world population is similar to that reported in the clinical trials) is rejected. The latter was performed overall and per regimen.

Next, a multivariable linear regression analysis was applied after log-transformation of the EE factor (because of the non-normal distribution) to study the association between the patient characteristics and the magnitude of the EE gap. All available characteristics were explored as potential prognostic factors. In this analysis, missing values were imputed by regression imputation (single run with all available characteristics in the model). The explanatory effect of the final model was assessed by variance analysis.

To assess the robustness of our main analysis, we conducted four sensitivity analyses: 1) calculating the real-world OS not from start of treatment, but based on date of diagnosis and based on start date of systemic treatment minus 14 days (this 2-week window was chosen because most clinical trial protocols require first drug administration within 2 weeks of randomization); 2) patients still alive at January 31, 2017 were given 1 year extra survival time (imputed date of death: January 31, 2018) instead of end of follow-up date; 3) replacing missing values in the variable ECOG PS by both the highest/worst value and the lowest/best value instead of the imputed value; and 4) a statistical bootstrap analysis to incorporate the uncertainty in the estimated pooled effect of the reference outcome for comparison with the real-world outcome. Therefore, a dataset was created by computerbased sampling (in Excel 2010; Microsoft, Redmond, WA, USA) with replacement from the original observations. The resampling and estimation procedure was repeated 10000 times, thus providing a distribution of the EE factor with a percentile-based confidence interval. 


\section{RESULTS}

From the Care for Outcome registry we were able to identify 2989 patients diagnosed with stage IV NSCLC in the period 2008-2014. After the exclusion of seven patients with vital status "emigrated", the final sample size was 2982 patients. Of these patients, 1214 (41\%) received first-line systemic treatment. Table 1 outlines the baseline patient characteristics per regimen and overall. The mean age was 63 years; $54 \%$ of all patients had a CCI of 0 (no comorbidities), and the majority of patients (84\%) had an ECOG PS of 0 or 1 at the time of diagnosis ( $5 \%$ missing data). The most frequently received chemotherapy regimen was cisplatin-pemetrexed (347 (29\%) out of 1214 patients), followed by cisplatin-gemcitabine $(n=214,18 \%)$, and carboplatin-pemetrexed $(n=213,18 \%)$. Epidermal growth factor receptor inhibitors (gefitinib and erlotinib) were used by $5 \%$ of the patients. Overall, eight regimens were responsible for $92 \%$ of the variety in applied first-line treatments ( $n=1122$ patients).

For all regimens, the median OS in the real-world setting is shorter than the clinical trial reference OS (Table 2), ranging from 1.3 to 7.7 months, depending on regimen. Overall, the distribution of the EE-factor is significantly different from a hypothesised median of 1.00 (median EE factor $0.77,95 \% \mathrm{Cl} 0.70-0.85 ; \mathrm{p}<0.001$ ), and the median EE factor is $<1.00$ for all individual treatment regimens (Figure 1).

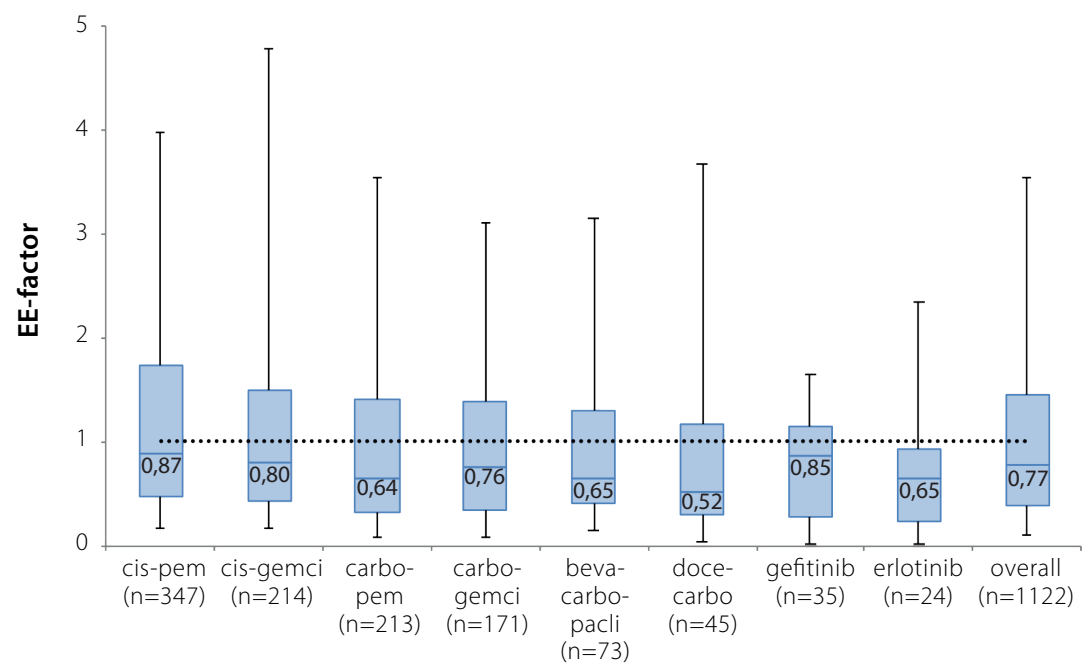

First-line systemic treatment regimen

Figure 1. Distribution of the efficacy-effectiveness (EE) factor based on start date of first-line systemic treatment, per regimen. For all regimens the median EE factor is significantly different from test value 1.0 (one sample Wilcoxon signed-rank test), with $p$-values of $p<0.05$. cis: cisplatin; pem: pemetrexed; gemci: gemcitabine; carbo: carboplatin; beva: bevacizumab; pacli: paclitaxel; doce: docetaxel. 
Table 1. Baseline characteristics of stage IV NSCLC patients and their first-line treatment

\begin{tabular}{|c|c|c|c|c|c|}
\hline & $\begin{array}{l}\text { Cisplatin- } \\
\text { pemetrexed }\end{array}$ & $\begin{array}{l}\text { Cisplatin- } \\
\text { gemcitabine }\end{array}$ & $\begin{array}{l}\text { Carboplatin- } \\
\text { pemetrexed }\end{array}$ & $\begin{array}{l}\text { Carboplatin- } \\
\text { gemcitabine }\end{array}$ & $\begin{array}{c}\text { Bevacizumab- } \\
\text { carboplatin- } \\
\text { paclitaxel }\end{array}$ \\
\hline Subjects & 347 (29) & $214(18)$ & $213(17)$ & $171(14)$ & $73(6)$ \\
\hline $\begin{array}{l}\text { Age at diagnosis } \\
\text { years }\end{array}$ & $59(9.7)$ & $63(9.3)$ & $65(8.8)$ & $68(8.0)$ & $62(9.0)$ \\
\hline Male & $171(49)$ & $127(59)$ & $132(62)$ & $123(72)$ & $35(48)$ \\
\hline \multicolumn{6}{|l|}{ Comorbidities (CCI) } \\
\hline 0 & $216(62)$ & $111(52)$ & $93(44)$ & $68(40)$ & $46(63)$ \\
\hline 1 & $76(22)$ & $60(28)$ & $62(29)$ & $51(30)$ & $19(26)$ \\
\hline $2-3$ & $50(14)$ & $41(19)$ & $54(25)$ & $50(29)$ & $8(11)$ \\
\hline$\geq 4$ & $5(1)$ & $2(1)$ & $4(2)$ & $2(1)$ & 0 \\
\hline \multicolumn{6}{|l|}{ ECOG PS } \\
\hline $0-1$ & $300(87)$ & $184(86)$ & $172(81)$ & $137(80)$ & $66(90)$ \\
\hline$\geq 2$ & $29(8)$ & $12(6)$ & $34(16)$ & $28(16)$ & $5(7)$ \\
\hline Missing & $18(5)$ & $18(8)$ & $7(3)$ & $6(4)$ & $2(3)$ \\
\hline \multicolumn{6}{|l|}{ Histology, No. (\%) } \\
\hline Squamous & $3(1)$ & $103(48)$ & $1(1)$ & $69(40)$ & 0 \\
\hline Adeno-carcinoma & $284(82)$ & $68(32)$ & $176(83)$ & $50(30)$ & $61(84)$ \\
\hline Large cell & $12(4)$ & $18(8)$ & $19(9)$ & $31(18)$ & $7(10)$ \\
\hline Other or NOS & $48(14)$ & $25(12)$ & $17(8)$ & $21(12)$ & $5(7)$ \\
\hline \multicolumn{6}{|l|}{ Year of diagnosis } \\
\hline 2008 & $12(4)$ & $49(23)$ & $2(1)$ & $25(15)$ & $1(1)$ \\
\hline 2009 & $46(3)$ & $34(16)$ & $15(7)$ & 31 (8) & 0 \\
\hline 2010 & $42(12)$ & 40 (19) & $36(17)$ & $15(9)$ & 0 \\
\hline 2011 & $62(18)$ & $22(10)$ & $30(14)$ & $21(12)$ & $24(33)$ \\
\hline 2012 & $63(18)$ & $18(8)$ & $45(21)$ & $26(15)$ & $22(30)$ \\
\hline 2013 & 65 (19) & $26(12)$ & $47(22)$ & $29(17)$ & $9(12)$ \\
\hline 2014 & $57(16)$ & $25(12)$ & 38 (18) & $24(14)$ & $17(23)$ \\
\hline
\end{tabular}

Data are presented as n (\%) or median \pm SD. EGFR: epidermal growth factor receptor; CCl: Charlson Comorbidity Index; ECOG PS: Eastern Cooperative Oncology Group performance status

The multivariable linear regression analysis showed that patient's ECOG PS is significantly associated with the magnitude of the EE factor ( $B-0.130,95 \% \mathrm{Cl}-0.163--0.098 ; p<0.001$ ). The negative B-value indicates a larger EE gap for patients with a higher/worse ECOG PS (category 0-4). The EE-gap was 19\% in patients with an ECOG PS of 0-1 (EE factor 0.81, $p<0.001$ ) and $61 \%$ in patients with ECOG PS $\geq 2$ (EE factor $0.39, p<0.001 ; n=140,12 \%$ ). The 


\section{Carboplatin-} docetaxel

Gefitinib
(with EGFR
mutation)

Erlotinib
(with EGFR
mutation)

\section{All treated patients}

Subjects
Age at diagnosis
years
Male
Comorbidities $(\mathrm{CCl})$

\section{0}

1

2-3

$\geq 4$

ECOG PS

$\begin{array}{lr}0-1 & 40(89) \\ \geq 2 & 4(9) \\ \text { Missing } & 1(2)\end{array}$

Histology, No. (\%)

Squamous
Adeno-carcinoma
Large cell

Other or NOS

\section{Year of diagnosis}

2008

2009

2010

2011

2012

2013

2014

$$
4 \text { (9) }
$$

23 (51)

9 (20)

9 (20)

$19(42)$

7 (16)

$10(22)$

8 (18)

1 (2)

0

0

$23(66)$
$8(23)$
$3(9)$
$1(3)$

31 (89)

4 (11)

0

$$
0
$$

32 (91)

0

3 (9)

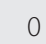

1 (3)

9 (26)

3 (9)

5 (14)

12 (34)

5 (14)

$24(2)$

68 (12.0)

6 (25)

16 (67)

5 (21)

3 (13)

0

$18(75)$

4 (17)

2 (8)

1 (4)

19 (79)

0

4 (17)

0

1 (4)

2 (8)

3 (13)

4 (17)

5 (21)

9 (38)

$\begin{array}{cc}92(7) & 1214(100) \\ 63(9.3) & 63(9.6)\end{array}$

$58(63)$

$690(57)$

650 (54)

317 (26)

227 (19)

20 (2)

5 (5)

1023 (84)

136 (11)

55 (5)

194 (16)

760 (63)

113 (9)

147 (12)

15 (16)

123 (10)

150 (12)

172 (14)

183 (15)

195 (16)

199 (16)

192 (16)

other variables in the model (age at diagnosis, sex, CCl (and separate comorbidities), histology, and year of diagnosis) showed no statistically significant association with the EE gap. However, ECOG PS alone explained only 5.2\% of the variation in EE factor.

The four sensitivity analyses confirmed the robustness of our findings. Independently of how the real-world OS was calculated, there was a significant divergence from the trial 
Table 2. Number of patients, real-world outcomes and reference outcome per regimen

\begin{tabular}{lccc}
\hline & $\begin{array}{c}\text { Patients } \\
\text { (real-world) } \\
\mathbf{n}\end{array}$ & $\begin{array}{c}\text { Median OS } \\
\text { (real-world) } \\
\text { months }\end{array}$ & $\begin{array}{c}\text { Median OS } \\
\text { (clinical trials) } \\
\text { months [reference] }\end{array}$ \\
\hline Cisplatin-pemetrexed & 347 & $8.90^{\mathrm{a}}$ & $10.19[14-17]$ \\
Cisplatin-gemcitabine & 214 & $7.90^{\mathrm{a}}$ & $9.88[17-44]$ \\
Carboplatin-pemetrexed & 213 & $6.51^{\mathrm{a}}$ & $10.18[45-49]$ \\
Carboplatin-gemcitabine & 171 & $6.67^{\mathrm{a}}$ & $8.75[43-45,50-60]$ \\
Bevacizumab-carboplatin-paclitaxel & 73 & $8.18^{\mathrm{a}}$ & $12.57[14,48,61,62]$ \\
Carboplatin-docetaxel & 45 & $4.93^{\mathrm{a}}$ & $9.52[46,63-66]$ \\
Gefitinib (in EGFR positive patients) & 35 & $21.19^{\mathrm{a}}$ & $24.90[67-72]$ \\
Erlotinib (in EGFR positive patients) & 24 & $14.32^{\mathrm{a}}$ & $22.01[73,74]$ \\
Total & $\mathbf{1 1 2 2}$ & $\mathbf{8 . 0 2}$ & $\mathbf{1 0 . 8 4}$ \\
\hline
\end{tabular}

OS: overall survival; EGFR: epidermal growth factor receptor. a: significantly different from median OS clinical trials

outcomes (the EE-factor based on date of diagnosis was 0.89 ( $p<0.001)$, and the EE factor based on start date of systemic treatment minus 14 days was $0.82(p<0.001))$. Furthermore, giving an additional 1 year survival time to patients still alive at January 31, 2017 resulted in a similar significant overall EE gap (EE factor of $0.77, \mathrm{p}<0.001$ ). Replacing missing values in the variable ECOG PS by both the highest/worst value (PS 4) and the lowest/best value (PS 0 ) instead of the imputed value did not change the finding that ECOG PS is associated with the magnitude of the EE-factor ( $B-0.083, p<0.001$ and $B-0.126, p<0.001$, respectively). Finally, the EE factors per chemotherapy regimen derived from the bootstrap analysis agreed very closely with the ones derived from the main statistical analysis; the same regimens appeared to have a statistical significant EE gap and the largest (although small) difference was for gefitinib (absolute difference of 0.03 between the original and the bootstrapped EE factor).

In real-world, patients completed their treatment plan less often than in clinical trials (56\% versus $61 \%, p=0.024$ ), and fewer patients received a subsequent line of chemotherapy in real-world compared to patients in clinical trials (34\% versus $46 \%, p<0.001$ ) (Table 3 ). The level of dose reductions ranged up to 39\% (carboplatin-gemcitabine) of the patients (Appendix 3). Overall, switching to another regimen due to toxicity occurred in $12 \%$ of the patients ( $n=131)$. By adding the variables "early discontinuation (<4 cycles)" and "subsequent line of chemotherapy" to the prognostic model for the EE factor, the explanatory effect of the model increased to $35 \%$. 
Table 3. Completion of treatment plan in first-line chemotherapy, and subsequent lines of chemotherapy

\begin{tabular}{|c|c|c|c|c|}
\hline & \multicolumn{2}{|c|}{ Completion of $\geq 4$ cycles } & \multicolumn{2}{|c|}{$\begin{array}{c}\text { Subsequent line of } \\
\text { chemotherapy }\end{array}$} \\
\hline & Real-world & $\begin{array}{l}\text { Clinical trials \% } \\
\text { (n references) }\end{array}$ & Real-world & $\begin{array}{c}\text { Clinical trials \% } \\
\text { (n references) }\end{array}$ \\
\hline Cisplatin-pemetrexed & $209(60)$ & $72(2)$ & $123(35)$ & $50(2)$ \\
\hline Cisplatin-gemcitabine & $129(60)$ & $44(19)$ & $79(37)$ & $43(10)$ \\
\hline Carboplatin-pemetrexed & $109(51)$ & $69(4)$ & $56(26)$ & $41(4)$ \\
\hline Carboplatin-gemcitabine & $76(44)$ & $58(13)$ & $56(33)$ & $21(5)$ \\
\hline $\begin{array}{l}\text { Bevacizumab- } \\
\text { carboplatin-paclitaxel }\end{array}$ & $52(71)$ & $70(2)$ & $29(40)$ & $54(3)$ \\
\hline Carboplatin-docetaxel & $22(49)$ & $52(4)$ & $19(42)$ & $33(4)$ \\
\hline $\begin{array}{l}\text { Gefitinib } \\
\text { (in EGFR positive patients) }\end{array}$ & 13.4 months $^{\mathrm{a}}$ & 7.0 months $^{\mathrm{a}}(3)$ & $16(46)$ & $66(5)$ \\
\hline $\begin{array}{l}\text { Erlotinib } \\
\text { (in EGFR positive patients) }\end{array}$ & 9.6 months $s^{a, b}$ & 10.5 months $^{\mathrm{a}}(2)$ & $9(38)$ & $57(1)$ \\
\hline Overall & $56 \%$ & $61 \%$ & $34 \%$ & $46 \%$ \\
\hline
\end{tabular}

Data are presented as $n$ (\%), unless otherwise stated. EGFR: epidermal growth factor receptor. a: mean duration of treatment in months; $\mathrm{b}: \mathrm{n}=1$ missing

\section{DISCUSSION}

This study showed that in patients with metastatic NSCLC treated with first-line systemic treatment, the median OS is nearly one-quarter shorter in real-world practice than in clinical trials (EE factor $0.77, \mathrm{p}<0.001$ ).

To our knowledge, this is the first study that provides a complete overview on the efficacyeffectiveness gap for different systemic treatments (chemotherapy and targeted therapy) in a large unselected population of metastatic NSCLC patients. The constant pattern of reduced effectiveness that we observed adds to the conclusion that the existence of a gap is a general phenomenon irrespective of the systemic treatment regimen. It also exists for targeted therapy.

The magnitude of the EE gap found in our study is in line with previous studies of routine care versus trials for specific regimens in metastatic NSCLC. Zhu et al. [5] observed a median survival estimate of 9.7 months for bevacizumab-carboplatin-paclitaxel in routine practice in stage IV NSCLC patients versus a median survival of 12.3 months for participants 
in the ECOG 4599 trial (equivalent to an EE factor of 0.79). The study by Sheikh and Chambers [7] showed that second-line treatment of advanced/metastatic NSCLC with erlotinib resulted in a median survival of 5.2 months in clinical practice, whereas the reference clinical trial reported a median OS of 6.7 months (EE factor 0.78 ).

In previous studies regarding prognostic factors in advanced NSCLC, ECOG PS has been shown to be an important independent prognostic parameter [11]. Patients with ECOG PS $\geq 2$ usually account for a small proportion of patients enrolled in trials of first-line treatment for advanced disease, but represent a significantly higher proportion (up to 30-40\%) when population-based surveys are conducted [11]. However, in our study population, the proportion of patients with ECOG PS $\geq 2$ was only $12.5 \%$, which is not very different from the clinical trials in our meta-analyses (15\%). This means that, although our data confirm performance status as prognostic factor for worse survival outcome, this is probably not the sole driver of the observed EE gap. Therefore, it needs to be associated with worse outcome in the real-world setting as well as being more prevalent in the real-world compared to a clinical trial. This means that other factors play a role. Two possible explanations from our data are the observed lower frequency of patients who received a subsequent line of treatment in the real-world population, and a lower percentage of patients who completed four or more cycles in the real-world population (addition of these two measures increased the explanatory effect of the model from 5\% to 35\%). These measures may possibly reflect a difference in comorbidity that influences vitality to undergo systemic treatment and/or selection towards more motivated patients in trials. Regarding comorbidities, in our dataset we observed no evident difference in frequencies between patients with or without early discontinuation of their systemic treatment (Appendix 3, Table S11). However, we acknowledge that the available comorbidity data are somewhat granular (e.g. chronic obstructive pulmonary disease (COPD) yes/no instead of COPD stages) and that the list of comorbidities is limited. The latter is supported by the results from the variance analysis, indicating that other unmeasured factors are involved. Other factors that are often mentioned in relation to an EE gap are the many exclusion criteria of clinical trials and that participating in a clinical trial itself also is beneficial (Hawthorne effect [12]). Unfortunately, these factors could not be assessed further in this project because the required data were not available in the Care for Outcome registry, nor are the required individual patient-level data from the corresponding clinical trials.

A strength of this study is that it is based on a large unselected population of patients diagnosed with stage IV NSCLC in the Netherlands, providing a general overview of most applied systemic treatment options and their outcomes in a western European country with a high standard level of healthcare. In addition, we captured a time frame of $>7$ years, reducing the risk for bias from temporal factors. Finally, this study is based on high-resolution data. The Care for Outcome registry includes detailed and validated patient data with a very low number of missing values (only one variable with $5 \%$ missing data). 
A limitation of this study could be our approach to calculate the real-world OS. Because most clinical trials calculate the OS from date of randomisation, our calculation based on date of first drug administration holds a risk of underestimating the OS. However, it is reassuring that our sensitivity analysis regarding this matter confirmed our main conclusion. Furthermore, one could argue about our approach to compare median OS between real-world and clinical trials primarily. An alternative way could be a hazard ratio based approach, but we considered this not feasible because of the unavailability of individual patient data from the included clinical trials and several other methodological drawbacks when it comes to extracting the necessary proxy data from published papers [13]. Regarding the consequence of not being able to censor patients in our approach, we consider the potential risk of bias rather small, because only $4 \%$ of the cohort was still alive at end of follow-up time. Our second sensitivity analysis based on a different imputed date of death for these patients also confirmed the absence of any substantial impact thereof. Thirdly, the reference median OS from the clinical trials might be overestimated, because of the allowance of patients with stage IIIB as well in almost all trials included in the meta-analysis. However, exclusion of trials with $>10 \%$ of stage IIIB patients from the meta-analysis did result in only very small alterations of the reference outcome, and with very little impact on the calculation of EE factors (overall 0.75, p<0.001). Finally, a drawback could be that the external validity of our findings might change due to the recent introduction of novel treatment options (e.g. immunotherapy and other targeted therapies) not covered by the time frame under study. Nevertheless, currently, the vast majority of patients receive one of the regimens studied in our project. Thus our findings have potential to improve shared decision-making by adding insight into real-world effectiveness data to the conversation. For example, the data can be used to inform a male patient with ECOG PS 1 planning to start with four cycles of cisplatin-pemetrexed that his OS is likely to be 10 weeks shorter than in the clinical trial report, and that preceding patients like him completed the four cycles in $60 \%$ of the cases.

In conclusion, our results show that patients treated in real-world practice have a nearly one-quarter shorter survival than those in clinical trials. The constant pattern of reduced effectiveness shows that the existence of this gap is a general phenomenon irrespective of the type of systemic treatment regimen. Patients' performance status, earlier discontinuation and fewer subsequent lines of treatment partly explain the difference. Clinical decisionmaking should be more strongly founded on real-world results.

\section{ACKNOWLEDGEMENTS}

The authors thank M. van Hulst (Martini Hospital, Groningen, The Netherlands), M.J. Deenen (Catharina Hospital, Eindhoven, The Netherlands), S.F. Oude Wesselink (Medisch Spectrum Twente, Enschede, The Netherlands), E.A.F. Haak (OLVG, Amsterdam, The 
Netherlands), and J. van der Mee (Canisius Wilhelmina Hospital, Nijmegen, The Netherlands) for their efforts to complete and validate the hospital pharmacy data. Furthermore, we thank R.H.H. Groenwold (University Medical Center Utrecht, The Netherlands) for assistance with the bootstrap procedure, and C. Sloof (St Antonius Hospital, Utrecht/Nieuwegein, The Netherlands) for literature search assistance. No one received financial compensation for these contributions. 


\section{REFERENCES}

1. Netherlands Cancer Registry, managed by Netherlands comprehensive cancer organisation, http://www.cijfersoverkanker.nl/. [cited Jan 23, 2017]; Available from: http://www.cijfersoverkanker.nl/

2. Molina JR, Yang P, Cassivi SD, Schild SE, Adjei AA. Non-small cell lung cancer: epidemiology, risk factors, treatment, and survivorship. Mayo Clin Proc 2008: 83(5): 584-594.

3. van der Drift MA, Karim-Kos HE, Siesling S, Groen HJ, Wouters MW, Coebergh JW, de Vries E, Janssen-Heijnen ML. Progress in standard of care therapy and modest survival benefits in the treatment of non-small cell lung cancer patients in the Netherlands in the last 20 years. J Thorac Oncol 2012: 7(2): 291-298.

4. Non-Small Cell Lung Cancer Collaborative G. Chemotherapy and supportive care versus supportive care alone for advanced non-small cell lung cancer. Cochrane Database Syst Rev 2010(5): CD007309.

5. Zhu J, Sharma DB, Gray SW, Chen AB, Weeks JC, Schrag D. Carboplatin and paclitaxel with vs without bevacizumab in older patients with advanced non-small cell lung cancer. JAMA 2012: 307(15): 1593-1601.

6. Jungels C, Berghmans T, Meert AP, Lafitte JJ, Scherpereel A, Sculier JP. [Pemetrexed salvage chemotherapy for NSCLC: implementation study]. Rev Mal Respir 2012: 29(1): 21-27.

7. Sheikh N, Chambers CR. Efficacy vs. effectiveness: erlotinib in previously treated non-small-cell lung cancer. J Oncol Pharm Pract 2013: 19(3): 228-236.

8. Peters BJ, Janssen VE, Schramel FM, van de Garde EM. Quantitative and qualitative assessment of real world data comparative effectiveness research of systemic therapies in lung oncology: A systematic review. Cancer Epidemiol 2016: 44: 5-15.

9. Peters BJM, Cramer-vd Welle CM, Smit AAJ, Schramel FMNH, van de Garde EMW. Trends in prescribing systemic treatment and overall survival for non-small cell lung cancer stage IIIB/IV in the Netherlands: 2008-2012. Cancer Epidemio/ 2017: 51:1-6.(doi).

10. van de Garde EMW, Plouvier BC, Fleuren HWHA, HaakEAF, Movig KLL, Deenen MJ, van Hulst M. Pharmacotherapy within a learning healthcare system: rationale for the Dutch Santeon Farmadatabase. European Journal of Hospital Pharmacy 2017: Published Online First: 18 September 2017.

11. Gridelli C, Ardizzoni A, Le Chevalier T, Manegold C, Perrone F, Thatcher N, van Zandwijk N, Di Maio M, Martelli O, De Marinis F. Treatment of advanced non-small-cell lung cancer patients with ECOG performance status 2: results of an European Experts Panel. Ann Oncol 2004: 15(3): 419-426.

12. McCambridge J, Witton J, Elbourne DR. Systematic review of the Hawthorne effect: new concepts are needed to study research participation effects. J Clin Epidemio/ 2014: 67(3): 267-277.

13. Piedbois P, Buyse M. Meta-analyses based on abstracted data: a step in the right direction, but only a first step. J Clin Oncol 2004: 22(19): 3839-3841.

14. Galetta D, Cinieri S, Pisconti S, Gebbia V, Morabito A, Borsellino N, Maiello E, Febbraro A, Catino A, Rizzo P, Montrone M, Misino A, Logroscino A, Rizzi D, Di Maio M, Colucci G. Cisplatin/pemetrexed followed by maintenance pemetrexed versus carboplatin/paclitaxel/bevacizumab followed by maintenance bevacizumab in advanced nonsquamous lung cancer: The GOIM (Gruppo Oncologico Italia Meridionale) ERACLE phase III randomized trial. Clinical Lung Cancer 2015: 16(4): 262-273.

15. Paz-Ares L, Mezger J, Ciuleanu TE, Fischer JR, von PJ, Provencio M, Kazarnowicz A, Losonczy G, de CG, Jr., Szczesna A, Crino L, Reck M, Ramlau R, Ulsperger E, Schumann C, Miziara JE, Lessa AE, Dediu M, Balint B, Depenbrock H, Soldatenkova V, Kurek R, Hirsch FR, Thatcher N, Socinski MA. Necitumumab plus pemetrexed and cisplatin as first-line therapy in patients with stage IV non-squamous non-small-cell lung cancer (INSPIRE): an open-label, randomised, controlled phase 3 study. Lancet Oncol 2015: 16(3): 328-337.

16. Scagliotti GV, Parikh P, von PJ, Biesma B, Vansteenkiste J, Manegold C, Serwatowski P, Gatzemeier U, Digumarti R, Zukin M, Lee JS, Mellemgaard A, Park K, Patil S, Rolski J, Goksel T, de MF, Simms L, Sugarman KP, Gandara D. Phase III study comparing cisplatin plus gemcitabine with cisplatin plus pemetrexed in chemotherapy-naive patients with advanced-stage non-small-cell lung cancer. J Clin Oncol 2008: 26(21): 3543-3551.

17. Lee SM, Falzon M, Blackhall F, Spicer J, Nicolson M, Chaudhuri A, Middleton G, Ahmed S, Hicks J, Crosse B, Napier M, Singer JM, Ferry D, Lewanski C, Forster M, Rolls SA, Capitanio A, Rudd R, lles N, Ngai Y, Gandy M, Lillywhite R, Hackshaw A. Randomized Prospective Biomarker Trial of ERCC1 for Comparing Platinum and Nonplatinum Therapy in Advanced Non-Small-Cell Lung Cancer: ERCC1 Trial (ET). J Clin Oncol 2017: 35(4): 402-411. doi: 410.1200/JCO.2016.1268.1841. 
18. Alberola V, Camps C, Provencio M, Isla D, Rosell R, Vadell C, Bover I, Ruiz-Casado A, Azagra P, Jim+ ${ }^{\oplus}$ nez U, Gonz+ílez-Larriba JL, Diz P, Cardenal F, Artal A, Carrato A, Morales S, Stínchez JJ, de Las Pe+łas R, Felip E, L+'pez-Vivanco G. Cisplatin plus gemcitabine versus a cisplatin-based triplet versus nonplatinum sequential doublets in advanced non-small-cell lung cancer: A Spanish Lung Cancer Group phase III randomized trial. Journal of Clinical Oncology 2003: 21(17): 3207-3213.

19. Bissett D, O'Byrne KJ, Von Pawel J, Gatzemeier U, Price A, Nicolson M, Mercier R, Mazabel E, Penning C, Zhang MH, Collier MA, Shepherd FA. Phase III study of matrix metalloproteinase inhibitor prinomastat in non-small-cell lung cancer. Journal of Clinical Oncology 2005: 23(4): 842-849.

20. Cardenal F, Lopez-Cabrerizo MP, Anton A, Alberola V, Massuti B, Carrato A, Barneto I, Lomas M, Garcia M, Lianes P, Montalar J, Vadell C, Gonzalez-Larriba JL, Nguyen B, Artal A, Rosell R. Randomized phase III study of gemcitabine-cisplatin versus etoposide-cisplatin in the treatment of locally advanced or metastatic non-small-cell lung cancer. J Clin Oncol 1999: 17(1): 12-18.

21. Chang JW-C, Tsao TC-Y, Yang CT, Lin MC, Cheung YC, Liaw CC, Chen CH. A randomized study of gemcitabine plus cisplatin and vinorelbine plus cisplatin in patients with advanced non-small-cell lung cancer. Chang Gung Medical Journal 2008: 31(6): 559-566

22. Crinò L, Scagliotti GV, Ricci S, De Marinis F, Rinaldi M, Gridelli C, Ceribelli A, Bianco R, Marangolo M, Di Costanzo F, Sassi M, Barni S, Ravaioli A, Adamo V, Portalone L, Cruciani G, Masotti A, Ferrara G, Gozzelino F, Tonato M. Gemcitabine and cisplatin versus mitomycin, ifosfamide, and cisplatin in advanced non-small-cell lung cancer: A randomized phase III study of the Italian Lung Cancer Project. Journal of Clinical Oncology 1999: 17(11): 3522-3530.

23. Gatzemeier U, Pluzanska A, Szczesna A, Kaukel E, Roubec J, De Rosa F, Milanowski J, Karnicka-Mlodkowski H, Pesek M, Serwatowski P, Ramlau R, Janaskova T, Vansteenkiste J, Strausz J, Manikhas GM, Von Pawel J. Phase III study of erlotinib in combination with cisplatin and gemcitabine in advanced non-small-cell lung cancer: The Tarceva lung cancer investigation trial. Journal of Clinical Oncology 2007: 25(12): 1545-1552.

24. Gebbia V, Galetta D, Caruso M, Verderame F, Pezzella G, Valdesi M, Borsellino N, Pandolfo G, Durini E, Rinaldi M, Loizzi M, Gebbia N, Valenza R, Tirrito ML, Varvara F, Colucci G. Gemcitabine and cisplatin versus vinorelbine and cisplatin versus ifosfamide+gemcitabine followed by vinorelbine and cisplatin versus vinorelbine and cisplatin followed by ifosfamide and gemcitabine in stage IIIB-IV non small cell lung carcinoma: A prospective randomized phase III trial of the Gruppo Oncologico Italia Meridionale. Lung Cancer 2003: 39(2): 179-189.

25. Giaccone G, Herbst RS, Manegold C, Scagliotti G, Rosell R, Miller V, Natale RB, Schiller JH, Von Pawel J, Pluzanska A, Gatzemeier U, Grous J, Ochs JS, Averbuch SD, Wolf MK, Rennie P, Fandi A, Johnson DH. Gefitinib in combination with gemcitabine and cisplatin in advanced non-small-cell lung cancer: A phase III trial - INTACT 1. Journal of Clinical Oncology 2004: 22(5): 777-784.

26. Gridelli C, Gallo C, Ceribelli A, Gebbia V, Gamucci T, Ciardiello F, Carozza F, Favaretto A, Daniele B, Galetta D, Barbera S, Rosetti F, Rossi A, Maione P, Cognetti F, Testa A, Di Maio M, Morabito A, Perrone F. Factorial phase III randomised trial of rofecoxib and prolonged constant infusion of gemcitabine in advanced non-small-cell lung cancer: the GEmcitabine-COxib in NSCLC (GECO) study. Lancet Oncology 2007: 8(6): 500-512.

27. Grigorescu A, Ciuleanu T, Firoiu E, Muresan DR, Teodorescu G, Basson BR. A randomized phase II trial of sequential gemcitabine plus vinorelbine followed by gemcitabine plus ifosfamide versus gemcitabine plus cisplatin in the treatment of chemo-naive patients with stages III and IV non-small cell lung cancer (NSCLC). Lung Cancer 2007: 57: 168-174.

28. Manegold C, van Zandwijk N, Szczesna A, Zatloukal P, Au JSK, Blasinska-Morawiec M, Serwatowski P, Krzakowski M, Jassem J, Tan EH, Benner RJ, Ingrosso A, Meech SJ, Readett D, Thatcher N. A phase III randomized study of gemcitabine and cisplatin with or without PF-3512676 (TLR9 agonist) as first-line treatment of advanced non-small-cell lung cancer. Annals of Oncology 2012: 23(1): 72-77.

29. Martoni A, Marino A, Sperandi F, Giaquinta S, Di Fabio F, Melotti B, Guaraldi M, Palomba G, Preti P, Petralia A, Artioli F, Picece V, Farris A, Mantovani L. Multicentre randomised phase III study comparing the same dose and schedule of cisplatin plus the same schedule of vinorelbine or gemcitabine in advanced non-small cell lung cancer. European Journal of Cancer 2005: 41(1): 81-92.

30. Morabito A, Gebbia V, Di Maio M, Cinieri S, Vigan+! MG, Bianco R, Barbera S, Cavanna L, De Marinis F, Montesarchio V, Costanzo R, Sandomenico C, Montanino A, Mancuso G, Russo P, Nacci A, Giordano P, Daniele G, Piccirillo MC, Rocco G, Gridelli C, Gallo C, Perrone F. Randomized phase III trial of gemcitabine and cisplatin 
vs. gemcitabine alone in patients with advanced non-small cell lung cancer and a performance status of 2: The CAPPA-2 study. Lung Cancer 2013: 81(1): 77-83.

31. Paz-Ares L, Douillard JY, Koralewski P, Manegold C, Smit EF, Reyes JM, Chang GC, John WJ, Peterson PM, Obasaju CK, Lahn M, Gandara DR. Phase III study of gemcitabine and cisplatin with or without aprinocarsen, a protein kinase C-alpha antisense oligonucleotide, in patients with advanced-stage non-small-cell lung cancer. Journal of Clinical Oncology 2006: 24(9): 1428-1434.

32. Paz-Ares LG, Biesma B, Heigener D, Von Pawel J, Eisen T, Bennouna J, Zhang L, Liao M, Sun Y, Gans S, Syrigos K, Le Marie E, Gottfried M, Vansteenkiste J, Alberola V, Strauss UP, Montegriffo E, Ong TJ, Santoro A. Phase III, randomized, double-blind, placebo-controlled trial of gemcitabine/cisplatin alone or with sorafenib for the first-line treatment of advanced, nonsquamous non-small-cell lung cancer. Journal of Clinical Oncology 2012: 30(25): 3084-3092.

33. Reck M, Von Pawel J, Zatloukal P, Ramlau R, Gorbounova V, Hirsh V, Leighl N, Mezger J, Archer V, Moore N, Manegold C. Phase III trial of cisplatin plus gemcitabine with either placebo or bevacizumab as first-line therapy for nonsquamous non-small-cell lung cancer: AVAiL. Journal of Clinical Oncology 2009: 27(8): 1227-1234.

34. Ridolfi L, Bertetto O, Santo A, Naglieri E, Lopez M, Recchia F, Lissoni P, Galliano M, Testore F, Porta C, Maglie M, Dall'Agata M, Fumagalli L, Ridolfi R. Chemotherapy with or without low-dose interleukin-2 in advanced non-small cell lung cancer: Results from a phase III randomized multicentric trial. International Journal of Oncology 2011: 39(4): 1011-1017.

35. Sandler AB, Nemunaitis J, Denham C, Von Pawel J, Cormier Y, Gatzemeier U, Mattson K, Manegold C, Palmer MC, Gregor A, Nguyen B, Niyikiza C, Einhorn LH. Phase III trial of gemcitabine plus cisplatin versus cisplatin alone in patients with locally advanced or metastatic non-small-cell lung cancer. Journal of Clinical Oncology 2000: 18(1): 122-130.

36. Scagliotti GV, De Marinis F, Rinaldi M, Crin+' L, Gridelli C, Ricci S, Matano E, Boni C, Marangolo M, Failla G, Altavilla G, Adamo V, Ceribelli A, Clerici M, Di Costanzo F, Frontini L, Tonato M. Phase III randomized trial comparing three platinum-based doublets in advanced non-small-cell lung cancer. Journal of Clinical Oncology 2002: 20(21): 4285-4291.

37. Scagliotti GV, Parikh P, Von Pawel J, Biesma B, Vansteenkiste J, Manegold C, Serwatowski P, Gatzemeier U, Digumarti R, Zukin M, Lee JS, Mellemgaard A, Park K, Patil S, Rolski J, Goksel T, De Marinis F, Simms L, Sugarman KP, Gandara D. Phase III study comparing cisplatin plus gemcitabine with cisplatin plus pemetrexed in chemotherapy-naive patients with advanced-stage non-small-cell lung cancer. Journal of Clinical Oncology 2008: 26(21): 3543-3551.

38. Schiller JH, Harrington D, Belani CP, Langer C, Sandler A, Krook J, Zhu J, Johnson DH. Comparison of four chemotherapy regimens for advanced non-small-cell lung cancer. New England Journal of Medicine 2002: 346(2): 92-98

39. Smit EF, Van Meerbeeck JPAM, Lianes P, Debruyne C, Legrand C, Schramel F, Smit H, Gaafar R, Biesma B, Manegold C, Neymark N, Giaccone G. Three-arm randomized study of two cisplatin-based regimens and paclitaxel plus gemcitabine in advanced non-small-cell lung cancer: A phase III trial of the European Organization for Research and Treatment of Cancer Lung Cancer Group - EORTC 08975. Journal of Clinical Oncology 2003: 21(21): 3909-3917.

40. Sweeney CJ, Zhu J, Sandler AB, Schiller J, Belani CP, Langer C, Krook J, Harrington D, Johnson DH. Outcome of patients with a performance status of 2 in Eastern Cooperative Oncology Group Study E1594: A phase III trial in patients with metastatic nonsmall cell lung carcinoma. Cancer 2001: 92(10): 2639-2647.

41. Thatcher N, Hirsch FR, Luft AV, Szczesna A, Ciuleanu TE, Dediu M, Ramlau R, Galiulin RK, B+ílint B, Losonczy G, Kazarnowicz A, Park K, Schumann C, Reck M, Depenbrock H, Nanda S, Kruljac-Letunic A, Kurek R, Paz-Ares L, Socinski MA. Necitumumab plus gemcitabine and cisplatin versus gemcitabine and cisplatin alone as first-line therapy in patients with stage IV squamous non-small-cell lung cancer (SQUIRE): An open-label, randomised, controlled phase 3 trial. The Lancet Oncology 2015: 16(7): 763-774.

42. Wachters FM, Van Putten JWJ, Kramer H, Erjavec Z, Eppinga P, Strijbos JH, De Leede GPJ, Boezen HM, De Vries EGE, Groen HJM. First-line gemcitabine with cisplatin or epirubicin in advanced non-small-cell lung cancer: A phase III trial. British Journal of Cancer 2003: 89(7): 1192-1199.

43. Zatloukal P, Petruželka L, Zemanová M, Kolek V, Skřičková J, Pešek M, Fojtů H, Grygárková I, Sixtová D, Roubec J, Hořenková E, Havel L, Průša P, Nováková L, Skácel T, Kůta M. Gemcitabine plus cisplatin vs. gemcitabine plus 
carboplatin in stage IIIb and IV non-small cell lung cancer: A phase III randomized trial. Lung Cancer 2003: 41(3): 321-331.

44. Ferry D, Billingham L, Jarrett H, Dunlop D, Woll PJ, Nicolson M, Shah R, Thompson J, Spicer J, Muthukumar D, Skailes G, Leonard P, Chetiyawardana AD, Wells P, Lewanski C, Crosse B, Hill M, Gaunt P, O'Byrne K. Carboplatin versus two doses of cisplatin in combination with gemcitabine in the treatment of advanced non-small-cell lung cancer: Results from a British Thoracic Oncology Group randomised phase III trial. Eur J Cancer 2017: 83:302-312.(doi): 10.1016/j.ejca.2017.1005.1037.

45. Grønberg BH, Bremnes RM, Fløtten $\varnothing$, Amundsen T, Brunsvig PF, Hjelde HH, Kaasa S, Von Plessen C, Stornes F, Tollåli T, Wammer F, Aasebø U, Sundstrøm S. Phase III study by the norwegian lung cancer study group: Pemetrexed plus carboplatin compared with gemcitabine plus carboplatin as first-line chemotherapy in advanced non-small-cell lung cancer. Journal of Clinical Oncology 2009: 27(19): 3217-3224.

46. Rodrigues-Pereira J, Kim JH, Magallanes M, Lee DH, Wang J, Ganju V, Mart+jnez-Barrera L, Barraclough H, Van Kooten M, Orlando M. A randomized phase 3 trial comparing pemetrexed/carboplatin and docetaxel/ carboplatin as first-line treatment for advanced, nonsquamous non-small cell lung cancer. Journal of Thoracic Oncology 2011: 6(11): 1907-1914.

47. Scagliotti GV, Kortsik C, Dark GG, Price A, Manegold C, Rosell R, O'Brien M, Peterson PM, Castellano D, Selvaggi G, Novello S, Blatter J, Kayitalire L, Crino L, Paz-Ares L. Pemetrexed combined with oxaliplatin or carboplatin as first-line treatment in advanced non-small cell lung cancer: a multicenter, randomized, phase II trial. Clin Cancer Res 2005: 11(2 Pt 1): 690-696.

48. Zinner RG, Obasaju CK, Spigel DR, Weaver RW, Beck JT, Waterhouse DM, Modiano MR, Hrinczenko B, Nikolinakos PG, Liu J, Koustenis AG, Winfree KB, Melemed SA, Guba SC, Ortuzar WI, Desaiah D, Treat JA, Govindan R, Ross HJ. PRONOUNCE: Randomized, open-label, phase III study of first-line pemetrexed + carboplatin followed by maintenance pemetrexed versus paclitaxel + carboplatin + bevacizumab followed by maintenance bevacizumab in patients ith advanced nonsquamous non-small-cell lung cancer. Journal of Thoracic Oncology 2015: 10(1): 134-142.

49. Zukin M, Barrios CH, Pereira JR, Ribeiro RDA, Beato CADM, Do Nascimento YN, Murad A, Franke FA, Precivale M, Araujo LHDL, Baldotto CSDR, Vieira FM, Small IA, Ferreira CG, Lilenbaum RC. Randomized phase III trial of single-agent pemetrexed versus carboplatin and pemetrexed in patients with advanced non-small-cell lung cancer and Eastern Cooperative Oncology Group performance status of 2. Journal of Clinical Oncology 2013: 31(23): 2849-2853.

50. Bepler G, Williams C, Schell MJ, Chen W, Zheng Z, Simon G, Gadgeel S, Zhao X, Schreiber F, Brahmer J, Chiappori A, Tanvetyanon T, Pinder-Schenck M, Gray J, Haura E, Antonia S, Fischer JR. Randomized international phase III trial of ERCC1 and RRM1 expression-based chemotherapy versus gemcitabine/carboplatin in advanced non-small-cell lung cancer. Journal of clinical oncology : official journal of the American Society of Clinical Oncology 2013: 31(19): 2404-2412.

51. Danson S, Middleton MR, O'Byrne KJ, Clemons M, Ranson M, Hassan J, Anderson H, Burt PA, Fairve-Finn C, Stout R, Dowd I, Ashcroft L, Beresford C, Thatcher N. Phase III trial of gemcitabine and carboplatin versus mitomycin, ifosfamide, and cisplatin or mitomycin, vinblastine, and cisplatin in patients with advanced nonsmall cell lung carcinoma. Cancer 2003: 98(3): 542-553.

52. Grigorescu AC, Draghici IN, Nitipir C, Gutulescu N, Corlan E. Gemcitabine (GEM) and carboplatin (CBDCA) versus cisplatin (CDDP) and vinblastine (VLB) in advanced non-small-cell lung cancer (NSCLC) stages III and IV: A phase III randomised trial. Lung Cancer 2002: 37(1): 9-14.

53. Helbekkmo N, Sundstrøm SH, Aasebø U, Fr Brunsvig P, Von Plessen C, Hjelde HH, Garpestad OK, Bailey A, Bremnes RM. Vinorelbine/carboplatin vs gemcitabine/carboplatin in advanced NSCLC shows similar efficacy, but different impact of toxicity. British Journal of Cancer 2007: 97(3): 283-289.

54. Kosmidis PA, Kalofonos HP, Christodoulou C, Syrigos K, Makatsoris T, Skarlos D, Bakogiannis C, Nicolaides C, Bafaloukos D, Bamias A, Samantas E, Xiros N, Boukovinas I, Fountzilas G, Dimopoulos MA. Paclitaxel and gemcitabine versus carboplatin and gemcitabine in patients with advanced non-small-cell lung cancer. $A$ phase III study of the Hellenic Cooperative Oncology Group. Annals of oncology: official journal of the European Society for Medical Oncology / ESMO 2008: 19(1): 115-122. 
55. Lee SM, Rudd R, Woll PJ, Ottensmeier C, Gilligan D, Price A, Spiro S, Gower N, Jitlal M, Hackshaw A. Randomized double-blind placebo-controlled trial of thalidomide in combination with gemcitabine and carboplatin in advanced non-small-cell lung cancer. Journal of Clinical Oncology 2009: 27(31): 5248-5254.

56. Reynolds C, Obasaju C, Schell MJ, Li X, Zheng Z, Boulware D, Caton JR, DeMarco LC, O'Rourke MA, Wright GS, Boehm KA, Asmar L, Bromund J, Peng G, Monberg MJ, Bepler G. Randomized phase III trial of gemcitabine-based chemotherapy with in situ RRM1 and ERCC1 protein levels for response prediction in non small-cell lung cancer. Journal of Clinical Oncology 2009: 27(34): 5808-5815.

57. Rudd RM, Gower NH, Spiro SG, Eisen TG, Harper PG, Littler JAH, Hatton M, Johnson PWM, Martin WMC, Rankin EM, James LE, Gregory WM, Qian W, Lee SM. Gemcitabine plus carboplatin versus mitomycin, ifosfamide, and cisplatin in patients with stage IIIB or IV non-small-cell lung cancer: A phase III randomized study of the London Lung Cancer Group. Journal of Clinical Oncology 2005: 23(1): 142-153.

58. Sederholm C, Hillerdal G, Lamberg K, K+Âlbeck K, Dufmats M, Westberg R, Gawande SR. Phase III trial of gemcitabine plus carboplatin versus single-agent gemcitabine in the treatment of locally advanced or metastatic non-small-cell lung cancer: The Swedish Lung Cancer Study Group. Journal of Clinical Oncology 2005: 23(33): 8380-8388.

59. Treat JA, Gonin R, Socinski MA, Edelman MJ, Catalano RB, Marinucci DM, Ansari R, Gillenwater HH, Rowland KM, Comis RL, Obasaju CK, Belani CP. A randomized, phase III multicenter trial of gemcitabine in combination with carboplatin or paclitaxel versus paclitaxel plus carboplatin in patients with advanced or metastatic non-small-cell lung cancer. Annals of Oncology 2010: 21(3): 540-547.

60. Biesma B, Wymenga AN, Vincent A, Dalesio O, Smit HJ, Stigt JA, Smit EF, van Felius CL, van Putten JW, Slaets JP, Groen HJ. Quality of life, geriatric assessment and survival in elderly patients with non-small-cell lung cancer treated with carboplatin-gemcitabine or carboplatin-paclitaxel: NVALT-3 a phase III study. Ann Oncol 2011: 22(7): 1520-1527. doi: 1510.1093/annonc/mdq1637.

61. Patel JD, Socinski MA, Garon EB, Reynolds CH, Spigel DR, Olsen MR, Hermann RC, Jotte RM, BeckT, Richards DA, Guba SC, Liu J, Frimodt-Moller B, John WJ, Obasaju CK, Pennella EJ, Bonomi P, Govindan R. PointBreak: A randomized phase III study of pemetrexed plus carboplatin and bevacizumab followed by maintenance pemetrexed and bevacizumab versus paclitaxel plus carboplatin and bevacizumab followed by maintenance bevacizumab in patients with stage IIIB or IV nonsquamous non-small-cell lung cancer. Journal of Clinical Oncology 2013: 31(34): 4349-4357.

62. Sandler A, Gray R, Perry MC, Brahmer J, Schiller JH, Dowlati A, Lilenbaum R, Johnson DH. Paclitaxel-carboplatin alone or with bevacizumab for non-small-cell lung cancer. New England Journal of Medicine 2006: 355(24): 2542-2550.

63. Booton R, Lorigan P, Anderson H, Baka S, Ashcroft L, Nicolson M, O'Brien M, Dunlop D, O'Byrne K, Laurence V, Snee M, Dark G, Thatcher N. A phase III trial of docetaxel/carboplatin versus mitomycin C/ifosfamide/ cisplatin (MIC) or mitomycin C/vinblastine/cisplatin (MVP) in patients with advanced non-small-cell lung cancer: A randomised multicentre trial of the British Thoracic Oncology Group (BTOG1). Annals of Oncology 2006: 17(7): 1111-1119.

64. Fossella F, Pereira JR, Von Pawel J, Pluzanska A, Gorbounova V, Kaukel E, Mattson KV, Ramlau R, Szcz+ ${ }^{\oplus}$ sna A, Fidias P, Millward M, Belani CP. Randomized, multinational, phase III study of docetaxel plus platinum combinations versus vinorelbine plus cisplatin for advanced non-small-cell lung cancer: The TAX 326 Study Group. Journal of Clinical Oncology 2003: 21(16): 3016-3024.

65. Groen HJM, Sietsma H, Vincent A, Hochstenbag MMH, Van Putten JWG, Van Den Berg A, Dalesio O, Biesma B, Smit HJM, Termeer A, Hiltermann TJN, Van Den Borne BEEM, Schramel FMNH. Randomized, placebo-controlled phase III study of docetaxel plus carboplatin with celecoxib and cyclooxygenase-2 expression as a biomarker for patients with advanced non-small-cell lung cancer: The NVALT-4 study. Journal of Clinical Oncology 2011: 29(32): 4320-4326.

66. Schuette W, Nagel S, Von Weikersthal LF, Pabst S, Schumann C, Deuss B, Salm T, Roscher K, Dickgreber N. Randomized phase III trial of docetaxel plus carboplatin with or without levofloxacin prophylaxis in elderly patients with advanced non-small cell lung cancer: The apronta trial. Journal of Thoracic Oncology 2011: 6(12): 2090-2096. 
67. An C, Zhang J, Chu H, Gu C, Xiao F, Zhu F, Lu R, Shi H, Zhang H, Yi X. Study of Gefitinib and Pemetrexed as First-Line Treatment in Patients with Advanced Non-Small Cell Lung Cancer Harboring EGFR Mutation. Pathology and Oncology Research 2016: 22(4): 763-768.

68. Han B, Jin B, Chu T, Niu Y, Dong Y, Xu J, Gu A, Zhong H, Wang H, Zhang X, Shi C, Zhang Y, Zhang W, Lou Y, Zhu $\mathrm{L}$, Pei J. Combination of chemotherapy and gefitinib as first-line treatment for patients with advanced lung adenocarcinoma and sensitive EGFR mutations: A randomized controlled trial. Int J Cancer 2017: 141(6): 1249-1256. doi: 1210.1002/ijc.30806.

69. Han JY, Park K, Kim SW, Lee DH, Kim HY, Kim HT, Ahn MJ, Yun T, Ahn JS, Suh C, Lee JS, Yoon SJ, Han JH, Lee JW, Jo SJ, Lee JS. First-SIGNAL: First-line single-agent iressa versus gemcitabine and cisplatin trial in never-smokers with adenocarcinoma of the lung. Journal of Clinical Oncology 2012: 30(10): 1122-1128.

70. Maemondo M, Inoue A, Kobayashi K, Sugawara S, Oizumi S, Isobe H, Gemma A, Harada M, Yoshizawa H, Kinoshita I, Fujita Y, Okinaga S, Hirano H, Yoshimori K, Harada T, Ogura T, Ando M, Miyazawa H, Tanaka T, Saijo Y, Hagiwara K, Morita S, Nukiwa T. Gefitinib or chemotherapy for non-small-cell lung cancer with mutated EGFR. New England Journal of Medicine 2010: 362(25): 2380-2388.

71. Mok TS, Wu YL, Thongprasert S, Yang CH, Chu DT, Saijo N, Sunpaweravong P, Han B, Margono B, Ichinose Y, Nishiwaki Y, Ohe Y, Yang JJ, Chewaskulyong B, Jiang H, Duffield EL, Watkins CL, Armour AA, Fukuoka M. Gefitinib or carboplatin-paclitaxel in pulmonary adenocarcinoma. New England Journal of Medicine 2009: 361(10): 947-957.

72. Patil VM, Noronha V, Joshi A, Choughule AB, Bhattacharjee A, Kumar R, Goud S, More S, Ramaswamy A, Karpe A, Pande N, Chandrasekharan A, Goel A, Talreja V, Mahajan A, Janu A, Purandare N, Prabhash K. Phase III study of gefitinib or pemetrexed with carboplatin in EGFR-mutated advanced lung adenocarcinoma. ESMO Open 2017: 2(1): e000168. doi: 000110.001136/esmoopen-002017-000168. eCollection 002017.

73. Rosell R, Carcereny E, Gervais R, Vergnenegre A, Massuti B, Felip E, Palmero R, Garcia-Gomez R, Pallares C, Sanchez JM, Porta R, Cobo M, Garrido P, Longo F, Moran T, Insa A, De Marinis F, Corre R, Bover I, Illiano A, Dansin E, de Castro J, Milella M, Reguart N, Altavilla G, Jimenez U, Provencio M, Moreno MA, Terrasa J, Mu+',oz-Langa J, Valdivia J, Isla D, Domine M, Molinier O, Mazieres J, Baize N, Garcia-Campelo R, Robinet G, Rodriguez-Abreu D, Lopez-Vivanco G, Gebbia V, Ferrera-Delgado L, Bombaron P, Bernabe R, Bearz A, Artal A, Cortesi E, Rolfo C, Sanchez-Ronco M, Drozdowskyj A, Queralt C, de Aguirre I, Ramirez JL, Sanchez JJ, Molina MA, Taron M, Paz-Ares L. Erlotinib versus standard chemotherapy as first-line treatment for European patients with advanced EGFR mutation-positive non-small-cell lung cancer (EURTAC): A multicentre, open-label, randomised phase 3 trial. The Lancet Oncology 2012: 13(3): 239-246.

74. Zhou C, Wu YL, Chen G, Feng J, Liu XQ, Wang C, Zhang S, Wang J, Zhou S, Ren S, Lu S, Zhang L, Hu C, Hu C, Luo Y, Chen L, Ye M, Huang J, Zhi X, Zhang Y, Xiu Q, Ma J, Zhang L, You C. Erlotinib versus chemotherapy as first-line treatment for patients with advanced EGFR mutation-positive non-small-cell lung cancer (OPTIMAL, CTONG-0802): a multicentre, open-label, randomised, phase 3 study. The Lancet Oncology 2011: 12(8): 735-742. 


\section{APPENDICES CHAPTER 3}

APPENDIX 1 Literature search

APPENDIX 2 Flow charts and reference tables clinical trial outcomes

STable 1. Clinical trial reference outcomes

SFigure 1. Flowchart cisplatin-pemetrexed

SFigure 2. Forest plot cisplatin-pemetrexed

STable 2. Clinical trial and real-world characteristics regimen cisplatin-pemetrexed

SFigure 3. Flowchart cisplatin-gemcitabine

SFigure 4. Forest plot cisplatin-gemcitabine

STable 3. Clinical trial and real-world characteristics regimen cisplatin-gemcitabine

SFigure 5. Flowchart carboplatin-pemetrexed

SFigure 6. Forest plot carboplatin-pemetrexed

STable 4. Clinical trial and real-world characteristics regimen carboplatin-pemetrexed

SFigure 7. Flowchart carboplatin-gemcitabine

SFigure 8. Forest plot carboplatin-gemcitabine

STable 5. Clinical trial and real-world characteristics regimen carboplatin-gemcitabine

SFigure 9. Flowchart carboplatin-paclitaxel-bevacizumab

SFigure 10. Forest plot carboplatin-paclitaxel-bevacizumab

STable 6. Clinical trial and real-world characteristics regimen carboplatin-paclitaxelbevacizumab

SFigure 11. Flowchart carboplatin-docetaxel

SFigure 12. Forest plot carboplatin-docetaxel

STable 7. Clinical trial and real-world characteristics regimen carboplatin-docetaxel

SFigure 13. Flowchart gefitinib

SFigure 14. Forest plot gefitinib

STable 8. Clinical trial and real-world characteristics regimen gefitinib

SFigure 15. Flowchart erlotinib

SFigure 16. Forest plot erlotinib

STable 9. Clinical trial and real-world characteristics regimen erlotinib

SFigure 17. Forest plot with overview of clinical trials for reference outcome first-line chemotherapy

APPENDIX 3 Proxies for toxicity

STable 10. Dose reduction and switches to other systemic agents as proxies for toxicity

STable 11. Co-morbidities in patients with early discontinuation of systemic therapy APPENDIX 4 List of included hospitals 


\section{APPENDIX 1 LITERATURE SEARCH}

\section{PubMed; search on September 1, 2017}

\section{Cisplatin pemetrexed}

((cisplatin*[tiab] OR "Cisplatin"[Mesh]) AND ("Pemetrexed"[Mesh] OR pemetrexed[tiab])) AND ("carcinoma, non-small-cell lung"[MeSH Terms] OR non small cell lung ca*[tiab] OR NSCLC [tiab] OR lung adenocarcinom*[tiab]) AND ("Neoplasm Metastasis"[Mesh] OR "secondary"[Subheading] OR stage IV[tiab] OR stage 4[tiab] OR stage IIIB[tiab] OR stage 3B[tiab] OR metastatic[tiab] OR metastas*[tiab] OR advanced[tiab])

AND ("mortality" [Subheading] OR "Mortality"[Mesh] OR "Survival"[Mesh] OR "Survival Analysis"[Mesh] OR mortality[tiab] OR death*[tiab] OR survival[tiab])

NOT ("animals"[mesh] NOT "humans"[mesh])

AND ("Clinical Trial, Phase III"[pt] OR phase III[ti] OR phase 3[ti] OR (("controlled clinical trial"[pt] OR controlled clinical trial[ti] OR randomized controlled trial[ti] OR randomized clinical trial[ti] OR randomised controlled trial[ti] OR randomised clinical trial[ti] OR rct[ti]) NOT ("Clinical Trial, Phase II"[pt] OR phase II[ti] OR phase 2[ti])))

AND english[la]

\section{Cisplatin gemcitabine}

((cisplatin*[tiab] OR "Cisplatin"[Mesh]) AND (gemcitabin*[tiab] OR "gemcitabine" [Supplementary Concept]))

AND ("carcinoma, non-small-cell lung"[MeSH Terms] OR non small cell lung ca*[tiab] OR NSCLC*[tiab] OR lung adenocarcinom*[tiab]) AND ("Neoplasm Metastasis"[Mesh] OR "secondary"[Subheading] OR stage IV[tiab] OR stage 4[tiab] OR stage IIIB[tiab] OR stage 3B[tiab] OR metastatic[tiab] OR metastas*[tiab] OR advanced[tiab])

AND ("mortality" [Subheading] OR "Mortality"[Mesh] OR "Survival"[Mesh] OR "Survival Analysis"[Mesh] OR mortality[tiab] OR death*[tiab] OR survival[tiab])

NOT ("animals"[mesh] NOT "humans"[mesh])

AND ("Clinical Trial, Phase III"[pt] OR phase III[ti] OR phase 3[ti] OR (("controlled clinical trial"[pt] OR controlled clinical trial[ti] OR randomized controlled trial[ti] OR randomized clinical trial[ti] OR randomised controlled trial[ti] OR randomised clinical trial[ti] OR rct[ti]) NOT ("Clinical Trial, Phase II"[pt] OR phase II[ti] OR phase 2[ti])))

AND english[la]

\section{Carboplatin pemetrexed}

((carboplatin*[tiab] OR "Carboplatin"[Mesh]) AND ("Pemetrexed"[Mesh] OR pemetrexed[tiab])) AND ("carcinoma, non-small-cell lung"[MeSH Terms] OR non small cell lung ca*[tiab] OR NSCLC [tiab] OR lung adenocarcinom*[tiab]) AND ("Neoplasm Metastasis"[Mesh] OR "secondary"[Subheading] OR stage IV[tiab] OR stage 4[tiab] OR stage IIIB[tiab] OR stage 3B[tiab] OR metastatic[tiab] OR metastas*[tiab] OR advanced[tiab]) 
AND ("mortality" [Subheading] OR "Mortality"[Mesh] OR "Survival"[Mesh] OR "Survival Analysis"[Mesh] OR mortality[tiab] OR death*[tiab] OR survival[tiab])

NOT ("animals"[mesh] NOT "humans"[mesh])

AND ("Clinical Trial, Phase III"[pt] OR phase III[ti] OR phase 3[ti] OR (("controlled clinical trial"[pt] OR controlled clinical trial[ti] OR randomized controlled trial[ti] OR randomized clinical trial[ti] OR randomised controlled trial[ti] OR randomised clinical trial[ti] OR rct[ti]) NOT ("Clinical Trial, Phase II"[pt] OR phase II[ti] OR phase 2[ti])))

AND english[la]

\section{Carboplatin gemcitabine}

((carboplatin*[tiab] OR "Carboplatin"[Mesh]) AND (gemcitabin*[tiab] OR "gemcitabine" [Supplementary Concept]))

AND ("carcinoma, non-small-cell lung"[MeSH Terms] OR non small cell lung ca*[tiab] OR NSCLC [tiab] OR lung adenocarcinom*[tiab]) AND ("Neoplasm Metastasis"[Mesh] OR "secondary" [Subheading] OR stage IV[tiab] OR stage 4[tiab] OR stage IIIB[tiab] OR stage 3B[tiab] OR metastatic[tiab] OR metastas*[tiab] OR advanced[tiab])

AND ("mortality" [Subheading] OR "Mortality"[Mesh] OR "Survival"[Mesh] OR "Survival Analysis"[Mesh] OR mortality[tiab] OR death*[tiab] OR survival[tiab])

NOT ("animals"[mesh] NOT "humans"[mesh])

AND ("Clinical Trial, Phase III"[pt] OR phase III[ti] OR phase 3[ti] OR (("controlled clinical trial"[pt] OR controlled clinical trial[ti] OR randomized controlled trial[ti] OR randomized clinical trial[ti] OR randomised controlled trial[ti] OR randomised clinical trial[ti] OR rct[ti]) NOT ("Clinical Trial, Phase II"[pt] OR phase II[ti] OR phase 2[ti])))

AND english[la]

\section{Bevacizumab carboplatin paclitaxel}

((bevacizumab[tiab] OR "Bevacizumab"[Mesh]) AND (carboplatin*[tiab] OR "Carboplatin"[Mesh]) AND (paclitaxel[tiab] OR "Paclitaxel"[Mesh]))

AND ("carcinoma, non-small-cell lung"[MeSH Terms] OR non small cell lung ca*[tiab] OR NSCLC*[tiab] OR lung adenocarcinom*[tiab]) AND ("Neoplasm Metastasis"[Mesh] OR "secondary"[Subheading] OR stage IV[tiab] OR stage 4[tiab] OR stage IIIB[tiab] OR stage 3B[tiab] OR metastatic[tiab] OR metastas*[tiab] OR advanced[tiab])

AND ("mortality" [Subheading] OR "Mortality"[Mesh] OR "Survival"[Mesh] OR "Survival Analysis"[Mesh] OR mortality[tiab] OR death*[tiab] OR survival[tiab])

NOT ("animals"[mesh] NOT "humans"[mesh])

AND ("Clinical Trial, Phase III"[pt] OR phase III[ti] OR phase 3[ti] OR (("controlled clinical trial"[pt] OR controlled clinical trial[ti] OR randomized controlled trial[ti] OR randomized clinical trial[ti] OR randomised controlled trial[ti] OR randomised clinical trial[ti] OR rct[ti]) NOT ("Clinical Trial, Phase II"[pt] OR phase II[ti] OR phase 2[ti])))

AND english[la] 


\section{Carboplatin docetaxel}

((carboplatin*[tiab] OR "Carboplatin"[Mesh]) AND ("docetaxel"[Supplementary Concept] OR docetaxel[tiab]))

AND ("carcinoma, non-small-cell lung"[MeSH Terms] OR non small cell lung ca*[tiab] OR NSCLC [tiab] OR lung adenocarcinom*[tiab]) AND ("Neoplasm Metastasis"[Mesh] OR "secondary"[Subheading] OR stage IV[tiab] OR stage 4[tiab] OR stage IIIB[tiab] OR stage 3B[tiab] OR metastatic[tiab] OR metastas*[tiab] OR advanced[tiab])

AND ("mortality" [Subheading] OR "Mortality"[Mesh] OR "Survival"[Mesh] OR "Survival Analysis"[Mesh] OR mortality[tiab] OR death*[tiab] OR survival[tiab])

NOT ("animals"[mesh] NOT "humans"[mesh])

AND ("Clinical Trial, Phase III"[pt] OR phase IIIti] OR phase 3[ti] OR (("controlled clinical trial"[pt] OR controlled clinical trial[ti] OR randomized controlled trial[ti] OR randomized clinical trial[ti] OR randomised controlled trial[ti] OR randomised clinical trial[ti] OR rct[ti]) NOT ("Clinical Trial, Phase II"[pt] OR phase II[ti] OR phase 2[ti])))

AND english[la]

\section{Gefitinib}

("gefitinib" [Supplementary Concept] OR gefitinib[tiab])

AND ("carcinoma, non-small-cell lung"[MeSH Terms] OR non small cell lung ca*[tiab] OR NSCLC [tiab] OR lung adenocarcinom*[tiab]) AND ("Neoplasm Metastasis"[Mesh] OR "secondary"[Subheading] OR stage IV[tiab] OR stage 4[tiab] OR stage IIIB[tiab] OR stage 3B[tiab] OR metastatic[tiab] OR metastas*[tiab] OR advanced[tiab])

AND ("mortality" [Subheading] OR "Mortality"[Mesh] OR "Survival"[Mesh] OR "Survival Analysis"[Mesh] OR mortality[tiab] OR death*[tiab] OR survival[tiab])

NOT ("animals"[mesh] NOT "humans"[mesh])

AND ("Clinical Trial, Phase III"[pt] OR phase III[ti] OR phase 3[ti] OR (("controlled clinical trial"[pt] OR controlled clinical trial[ti] OR randomized controlled trial[ti] OR randomized clinical trial[ti] OR randomised controlled trial[ti] OR randomised clinical trial[ti] OR rct[ti]) NOT ("Clinical Trial, Phase II"[pt] OR phase II[ti] OR phase 2[ti])))

AND english[la]

\section{Erlotinib}

("Erlotinib Hydrochloride"[Mesh] OR erlotinib[tiab])

AND ("carcinoma, non-small-cell lung"[MeSH Terms] OR non small cell lung ca*[tiab] OR NSCLC*[tiab] OR lung adenocarcinom*[tiab]) AND ("Neoplasm Metastasis"[Mesh] OR "secondary"[Subheading] OR stage IV[tiab] OR stage 4[tiab] OR stage IIIB[tiab] OR stage 3B[tiab] OR metastatic[tiab] OR metastas*[tiab] OR advanced[tiab])

AND ("mortality" [Subheading] OR "Mortality"[Mesh] OR "Survival"[Mesh] OR "Survival Analysis"[Mesh] OR mortality[tiab] OR death*[tiab] OR survival[tiab])

NOT ("animals"[mesh] NOT "humans"[mesh]) 
AND ("Clinical Trial, Phase III"[pt] OR phase III[ti] OR phase 3[ti] OR (("controlled clinical trial"[pt] OR controlled clinical trial[ti] OR randomized controlled trial[ti] OR randomized clinical trial[ti] OR randomised controlled trial[ti] OR randomised clinical trial[ti] OR rct[ti]) NOT ("Clinical Trial, Phase II"[pt] OR phase II[ti] OR phase 2[ti])))

AND english[la]

\section{Embase via Embase.com; search on September 1, 2017}

\section{Cisplatin pemetrexed}

(('cisplatin'/exp OR cisplatin*:ab,ti) AND ('pemetrexed'/exp OR pemetrexed:ab,ti)) AND ('non small cell lung cancer'/exp OR 'non small cell lung carcinoma':ab,ti OR 'non small cell lung cancer':ab,ti OR NSCLC*:ab,ti OR 'lung adenocarcinoma':ab,ti) AND ('metastasis'/exp OR 'stage IV':ab,ti OR 'stage 4':ab,ti OR 'stage IIIB':ab,ti OR 'stage 3B':ab,ti OR metastatic:ab,ti OR metastas*:ab,ti OR advanced:ab,ti) AND ('mortality'/exp OR 'survival'/exp OR mortality: ab,ti OR death*:ab,ti OR survival:ab,ti) AND ('phase 3 clinical trial'/exp OR 'phase III':ti OR 'phase 3':ti OR 'controlled clinical trial'/exp OR 'controlled clinical trial':ti OR 'randomized controlled trial':ti OR 'randomized clinical trial':ti OR 'randomised controlled trial':ti OR 'randomised clinical trial':ti OR rct:ti NOT ('phase 2 clinical trial'/exp OR 'phase Il':ti OR 'phase 2':ti)) AND [english]/lim NOT 'conference abstract'/it

\section{Cisplatin gemcitabine}

('cisplatin'/exp OR cisplatin*:ab,ti) AND ('gemcitabine'/exp OR gemcitabin*:ab,ti)) AND ('non small cell lung cancer'/exp OR 'non small cell lung carcinoma':ab,ti OR 'non small cell lung cancer':ab,ti OR NSCLC*:ab,ti OR 'lung adenocarcinoma':ab,ti) AND ('metastasis'/exp OR 'stage IV':ab,ti OR 'stage 4':ab,ti OR 'stage IIIB':ab,ti OR 'stage 3B':ab,ti OR metastatic:ab,ti OR metastas*:ab,ti OR advanced:ab,ti) AND ('mortality'/exp OR 'survival'/exp OR mortality: ab,ti OR death*:ab,ti OR survival:ab,ti) AND ('phase 3 clinical trial'/exp OR 'phase III':ti OR 'phase 3':ti OR 'controlled clinical trial'/exp OR 'controlled clinical trial':ti OR 'randomized controlled trial':ti OR 'randomized clinical trial':ti OR 'randomised controlled trial':ti OR 'randomised clinical trial':ti OR rct:ti NOT ('phase 2 clinical trial'/exp OR 'phase Il':ti OR 'phase 2':ti)) AND [english]/lim NOT 'conference abstract'/it

\section{Carboplatin pemetrexed}

(('carboplatin'/exp OR carboplatin*:ab,ti) AND ('pemetrexed'/exp OR pemetrexed:ab,ti)) AND ('non small cell lung cancer'/exp OR 'non small cell lung carcinoma':ab,ti OR 'non small cell lung cancer':ab,ti OR NSCLC*ab,ti OR 'lung adenocarcinoma':ab,ti) AND ('metastasis'/exp OR 'stage IV':ab,ti OR 'stage 4':ab,ti OR 'stage III':ab,ti OR 'stage 3B':ab,ti OR metastatic:ab,ti OR metastas*:ab,ti OR advanced:ab,ti) AND ('mortality'/exp OR 'survival') exp OR mortality:ab,ti OR death*:ab,ti OR survival:ab,ti) AND ('phase 3 clinical trial'/exp OR 'phase III':ti OR 'phase 3':ti OR 'controlled clinical trial'/exp OR 'controlled clinical trial':ti OR 'randomized controlled trial':ti OR 'randomized clinical trial':ti OR 'randomised controlled 
trial':ti OR 'randomised clinical trial':ti OR rct:ti NOT ('phase 2 clinical trial'/exp OR 'phase Il':ti OR 'phase 2':ti)) AND [english]/lim NOT 'conference abstract'/it

\section{Carboplatin gemcitabine}

(('carboplatin'/exp OR carboplatin*:ab,ti) AND ('gemcitabine'/exp OR gemcitabin*:ab,ti)) AND ('non small cell lung cancer'/exp OR 'non small cell lung carcinoma':ab,ti OR 'non small cell lung cancer':ab,ti OR NSCLC*:ab,ti OR 'lung adenocarcinoma':ab,ti) AND ('metastasis'/exp OR 'stage IV':ab,ti OR 'stage 4':ab,ti OR 'stage IIIB':ab,ti OR 'stage 3B':ab,ti OR metastatic:ab,ti OR metastas*:ab,ti OR advanced:ab,ti) AND ('mortality'/exp OR 'survival'/ exp OR mortality:ab,ti OR death*:ab,ti OR survival:ab,ti) AND ('phase 3 clinical trial'/exp OR 'phase III':ti OR 'phase 3':ti OR 'controlled clinical trial'/exp OR 'controlled clinical trial':ti OR 'randomized controlled trial':ti OR 'randomized clinical trial':ti OR 'randomised controlled trial':ti OR 'randomised clinical trial':ti OR rct:ti NOT ('phase 2 clinical trial'/exp OR 'phase Il':ti OR 'phase 2':ti)) AND [english]/lim NOT 'conference abstract'/it

\section{Bevacizumab carboplatin paclitaxel}

(('bevacizumab'/exp OR bevacizumab:ab,ti) AND ('carboplatin'/exp OR carboplatin*:ab,ti) AND ('paclitaxel'/exp OR paclitaxel:ab,ti)) AND ('non small cell lung cancer'/exp OR 'non small cell lung carcinoma':ab,ti OR 'non small cell lung cancer':ab,ti OR NSCLC*ab,ti OR 'lung adenocarcinoma':ab,ti) AND ('metastasis'/exp OR 'stage IV':ab,ti OR 'stage 4':ab,ti OR 'stage III' 'ab,ti OR 'stage 3B':ab,ti OR metastatic:ab,ti OR metastas*:ab,ti OR advanced:ab,ti) AND ('mortality'/exp OR 'survival'/exp OR mortality:ab,ti OR death*:ab,ti OR survival:ab,ti) AND ('phase 3 clinical trial'/exp OR 'phase III':ti OR 'phase 3':ti OR 'controlled clinical trial'/ exp OR 'controlled clinical trial':ti OR 'randomized controlled trial':ti OR 'randomized clinical trial':ti OR 'randomised controlled trial':ti OR 'randomised clinical trial':ti OR rct:ti NOT ('phase 2 clinical trial'/exp OR 'phase Il':ti OR 'phase 2':ti)) AND [english]/lim NOT 'conference abstract'/it

\section{Carboplatin docetaxel}

(('carboplatin'/exp OR carboplatin*:ab,ti) AND ('docetaxel'/exp OR docetaxel:ab,ti)) AND ('non small cell lung cancer'/exp OR 'non small cell lung carcinoma':ab,ti OR 'non small cell lung cancer':ab,ti OR NSCLC :ab,ti OR 'lung adenocarcinoma':ab,ti) AND ('metastasis'/exp OR 'stage IV':ab,ti OR 'stage 4':ab,ti OR 'stage IIIB':ab,ti OR 'stage 3B':ab,ti OR metastatic:ab,ti OR metastas*:ab,ti OR advanced:ab,ti) AND ('mortality'/exp OR 'survival'/exp OR mortality:ab,ti OR death*:ab,ti OR survival:ab,ti) AND ('phase 3 clinical trial'/exp OR 'phase III':ti OR 'phase 3':ti OR 'controlled clinical trial'/exp OR 'controlled clinical trial':ti OR 'randomized controlled trial':ti OR 'randomized clinical trial':ti OR 'randomised controlled trial':ti OR 'randomised clinical trial':ti OR rct:ti NOT ('phase 2 clinical trial'/exp OR 'phase Il':ti OR 'phase 2':ti)) AND [english]/lim NOT 'conference abstract'/it 


\section{Gefitinib}

('gefitinib'/exp OR gefitinib:ab,ti) AND ('non small cell lung cancer'/exp OR 'non small cell lung carcinoma':ab,ti OR 'non small cell lung cancer':ab,ti OR NSCLC*:ab,ti OR 'lung adenocarcinoma':ab,ti) AND ('metastasis'/exp OR 'stage IV':ab,ti OR 'stage 4':ab,ti OR 'stage IIIB':ab,ti OR 'stage 3B':ab,ti OR metastatic:ab,ti OR metastas*:ab,ti OR advanced:ab,ti) AND ('mortality'/exp OR 'survival'/exp OR mortality:ab,ti OR death*:ab,ti OR survival:ab,ti) AND ('phase 3 clinical trial'/exp OR 'phase III':ti OR 'phase 3':ti OR 'controlled clinical trial'/exp OR 'controlled clinical trial':ti OR 'randomized controlled trial':ti OR 'randomized clinical trial':ti OR 'randomised controlled trial':ti OR 'randomised clinical trial':ti OR rct:ti NOT ('phase 2 clinical trial'/exp OR 'phase Il':ti OR 'phase 2':ti)) AND [english]/lim NOT 'conference abstract'/it

\section{Erlotinib}

('erlotinib'/exp OR erlotinib:ab,ti) AND ('non small cell lung cancer'/exp OR 'non small cell lung carcinoma':ab,ti OR 'non small cell lung cancer':ab,ti OR NSCLC*:ab,ti OR 'lung adenocarcinoma':ab,ti) AND ('metastasis'/exp OR 'stage IV':ab,ti OR 'stage 4':ab,ti OR 'stage III':ab,ti OR 'stage 3B':ab,ti OR metastatic:ab,ti OR metastas*:ab,ti OR advanced:ab,ti) AND ('mortality'/exp OR 'survival'/exp OR mortality:ab,ti OR death*:ab,ti OR survival:ab,ti) AND ('phase 3 clinical trial'/exp OR 'phase III':ti OR 'phase 3':ti OR 'controlled clinical trial'/exp OR 'controlled clinical trial':ti OR 'randomized controlled trial':ti OR 'randomized clinical trial':ti OR 'randomised controlled trial':ti OR 'randomised clinical trial':ti OR rct:ti NOT ('phase 2 clinical trial'/exp OR 'phase Il':ti OR 'phase 2':ti)) AND [english]/lim NOT 'conference abstract'/it

\section{CENTRAL (Cochrane library); search on September 1, 2017}

\section{Cisplatin pemetrexed}

(cisplatin*:ab,ti AND pemetrexed:ab,ti) AND ('non small cell lung carcinoma':ab,ti OR 'non small cell lung cancer':ab,ti OR NSCLC*ab,ti OR 'lung adenocarcinoma':ab,ti) AND ('stage IV':ab,ti OR 'stage 4':ab,ti OR 'stage IIIB':ab,ti OR 'stage 3B':ab,ti OR metastatic:ab,ti OR metastas*:ab,ti OR advanced:ab,ti) AND (mortality:ab,ti OR death*:ab,ti OR survival:ab,ti) AND ('phase 3':ti OR 'phase III':ti)

\section{Cisplatin gemcitabine}

(cisplatin*:ab,ti AND gemcitabin*:ab,ti) AND ('non small cell lung carcinoma':ab,ti OR 'non small cell lung cancer':ab,ti OR NSCLC*ab,ti OR 'lung adenocarcinoma':ab,ti) AND ('stage IV':ab,ti OR 'stage 4':ab,ti OR 'stage IIIB':ab,ti OR 'stage 3B':ab,ti OR metastatic:ab,ti OR metastas*ab,ti OR advanced:ab,ti) AND (mortality:ab,ti OR death*:ab,ti OR survival:ab,ti) AND ('phase 3':ti OR 'phase III':ti)

\section{Carboplatin pemetrexed}

(carboplatin*:ab,ti AND pemetrexed:ab,ti) AND ('non small cell lung carcinoma':ab,ti OR 'non small cell lung cancer':ab,ti OR NSCLC :ab,ti OR 'lung adenocarcinoma':ab,ti) AND 
('stage IV':ab,ti OR 'stage 4':ab,ti OR 'stage IIIB':ab,ti OR 'stage 3B':ab,ti OR metastatic:ab,ti OR metastas*:ab,ti OR advanced:ab,ti) AND (mortality:ab,ti OR death*:ab,ti OR survival:ab,ti) AND ('phase 3':ti OR 'phase III':ti)

\section{Carboplatin gemcitabine}

(carboplatin*:ab,ti AND gemcitabin*:ab,ti) AND ('non small cell lung carcinoma':ab,ti OR 'non small cell lung cancer':ab,ti OR NSCLC:ab,ti OR 'lung adenocarcinoma':ab,ti) AND ('stage IV':ab,ti OR 'stage 4':ab,ti OR 'stage III' 'ab,ti OR 'stage 3B':ab,ti OR metastatic:ab,ti OR metastas*ab,ti OR advanced:ab,ti) AND (mortality:ab,ti OR death*ab,ti OR survival:ab,ti) AND ('phase 3':ti OR 'phase III':ti)

\section{Bevacizumab carboplatin paclitaxel}

(bevacizumab:ab,ti AND carboplatin*:ab,ti AND paclitaxel:ab,ti) AND ('non small cell lung carcinoma':ab,ti OR 'non small cell lung cancer':ab,ti OR NSCLC*:ab,ti OR 'lung adenocarcinoma':ab,ti) AND ('stage IV':ab,ti OR 'stage 4':ab,ti OR 'stage III':ab,ti OR 'stage 3B':ab,ti OR metastatic:ab,ti OR metastas*:ab,ti OR advanced:ab,ti) AND (mortality:ab,ti OR death*ab,ti OR survival:ab,ti) AND ('phase 3':ti OR 'phase III':ti)

\section{Carboplatin docetaxel}

(carboplatin*:ab,ti AND docetaxel:ab,ti) AND ('non small cell lung carcinoma':ab,ti OR 'non small cell lung cancer':ab,ti OR NSCLC*:ab,ti OR 'lung adenocarcinoma':ab,ti) AND ('stage IV':ab,ti OR 'stage 4':ab,ti OR 'stage III':ab,ti OR 'stage 3B':ab,ti OR metastatic:ab,ti OR metastas*:ab,ti OR advanced:ab,ti) AND (mortality:ab,ti OR death*:ab,ti OR survival:ab,ti) AND ('phase 3':ti OR 'phase III':ti)

\section{Gefitinib}

(gefitinib:ab,ti) AND ('non small cell lung carcinoma':ab,ti OR 'non small cell lung cancer':ab,ti OR NSCLC*:ab,ti OR 'lung adenocarcinoma':ab,ti) AND ('stage IV':ab,ti OR 'stage 4':ab,ti OR 'stage III' 'ab,ti OR 'stage 3B':ab,ti OR metastatic:ab,ti OR metastas*:ab,ti OR advanced:ab,ti) AND (mortality:ab,ti OR death*:ab,ti OR survival:ab,ti) AND ('phase 3':ti OR 'phase III':ti)

\section{Erlotinib}

(erlotinib:ab,ti) AND ('non small cell lung carcinoma':ab,ti OR 'non small cell lung cancer':ab,ti OR NSCLC*:ab,ti OR 'lung adenocarcinoma':ab,ti) AND ('stage IV':ab,ti OR 'stage 4':ab,ti OR 'stage III' AND (mortality:ab,ti OR death*:ab,ti OR survival:ab,ti) AND ('phase 3':ti OR 'phase III'ti) 


\section{APPENDIX 2 FLOW CHARTS AND REFERENCE TABLES CLINICAL TRIAL OUTCOMES}

STable 1. Clinical trial reference outcomes

\begin{tabular}{llc}
\hline Chemotherapy regimen & $\begin{array}{c}\text { Median OS clinical trials } \\
\text { (months) }\end{array}$ \\
\hline 1. & Cisplatin pemetrexed & 10.19 \\
2. & Cisplatin gemcitabine & 9.88 \\
3. $\quad$ Carboplatin pemetrexed & 10.18 \\
4. $\quad$ Carboplatin gemcitabine & 8.75 \\
5. $\quad$ Bevacizumab carboplatin paclitaxel & 12.57 \\
6. Carboplatin docetaxel & 9.52 \\
7. Gefitinib (in EGFR-mutation positive patients) & 24.90 \\
8. $\quad$ Erlotinib (in EGFR-mutation positive patients) & 22.01 \\
\multicolumn{2}{l}{ Overall } & $\mathbf{1 0 . 8 4}$ \\
\hline
\end{tabular}




\section{Cisplatin pemetrexed}

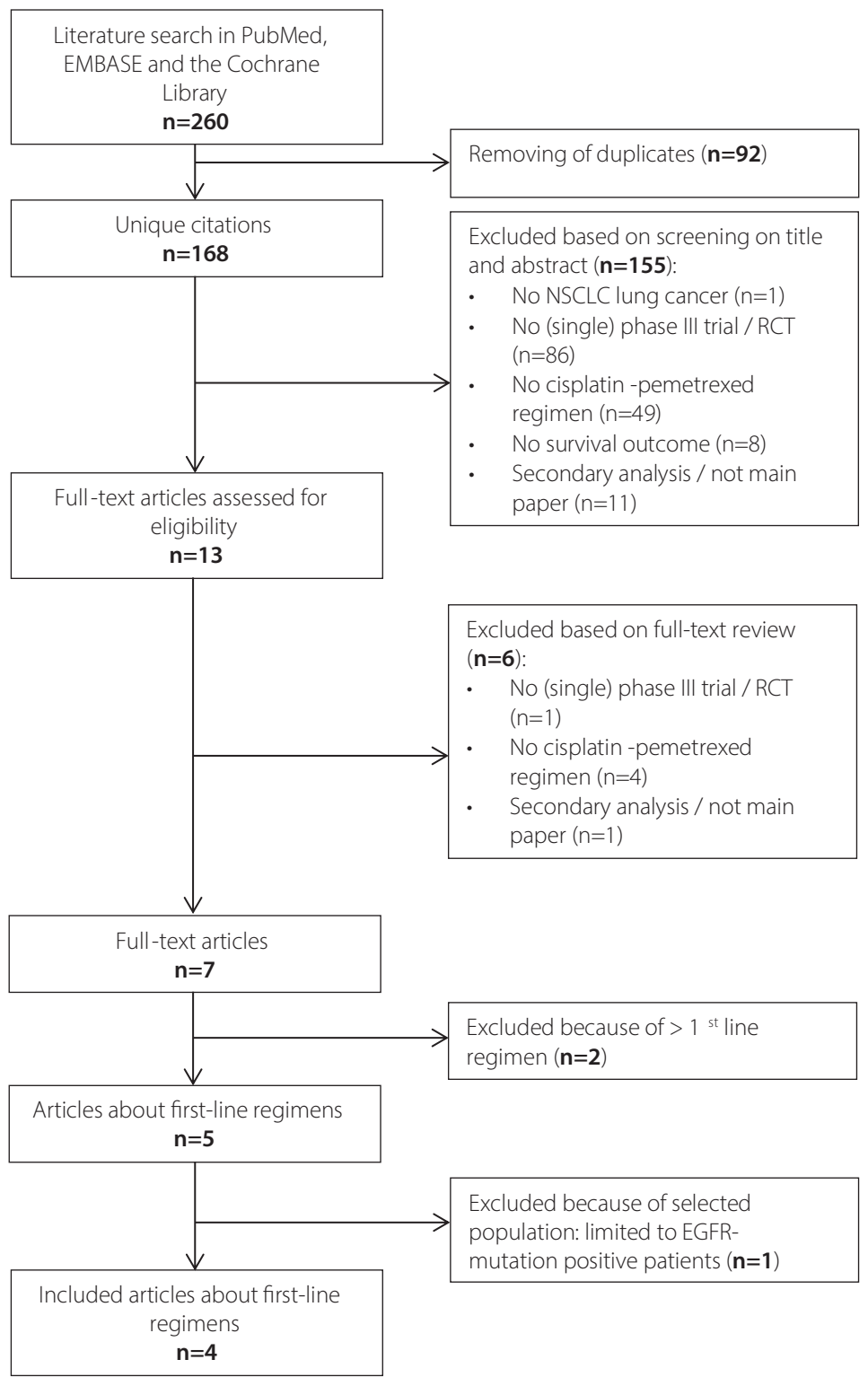

SFigure 1. Flowchart cisplatin-pemetrexed 


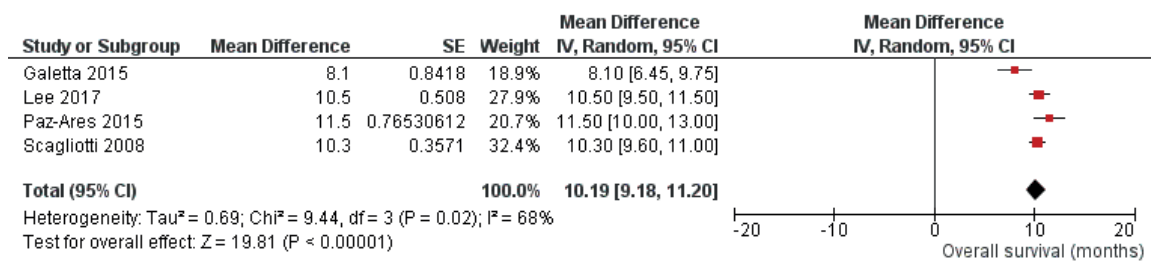

SFigure 2. Forest plot cisplatin-pemetrexed

STable 2. Clinical trial and real-world characteristics regimen cisplatin-pemetrexed

\begin{tabular}{lcc}
\hline Characteristic & Clinical trials $^{\mathbf{a}}$ & Real-world \\
\hline Total number of patients & 1470 & 347 \\
Overall survival (months) & 10.19 & 8.90 \\
Median age (years) & 60.6 & 59 \\
Male (\%) & 66.1 & 49 \\
Stage IV (\%) & 87.8 & 100 \\
ECOG Performance Status & & \\
ECOG PS 0-1 (\%) & 98.5 & 87 \\
ECOG PS $\geq 2$ (\%) & 1.5 & 5 \\
Missing & --- & 1 \\
Histology & & 82 \\
squamous (\%) & 9.4 & 4 \\
adenocarcinoma (\%) & 79.2 & 14 \\
large-cell (\%) & 6.6 & \\
other or unknown histology (\%) & 4.8 & \\
\hline
\end{tabular}

aHistology based on 3 references 


\section{Cisplatin gemcitabine}

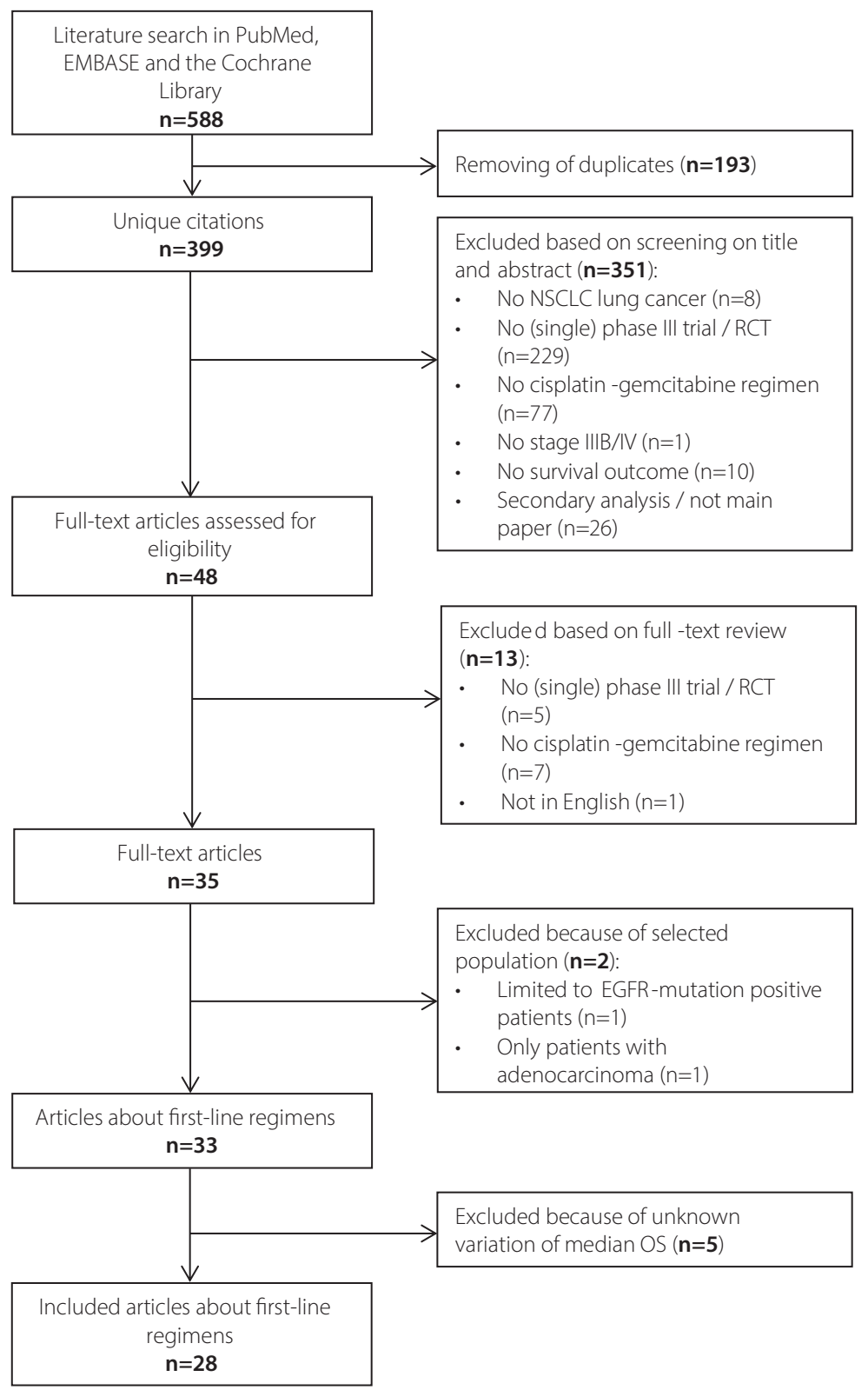

SFigure 3. Flowchart cisplatin-gemcitabine 


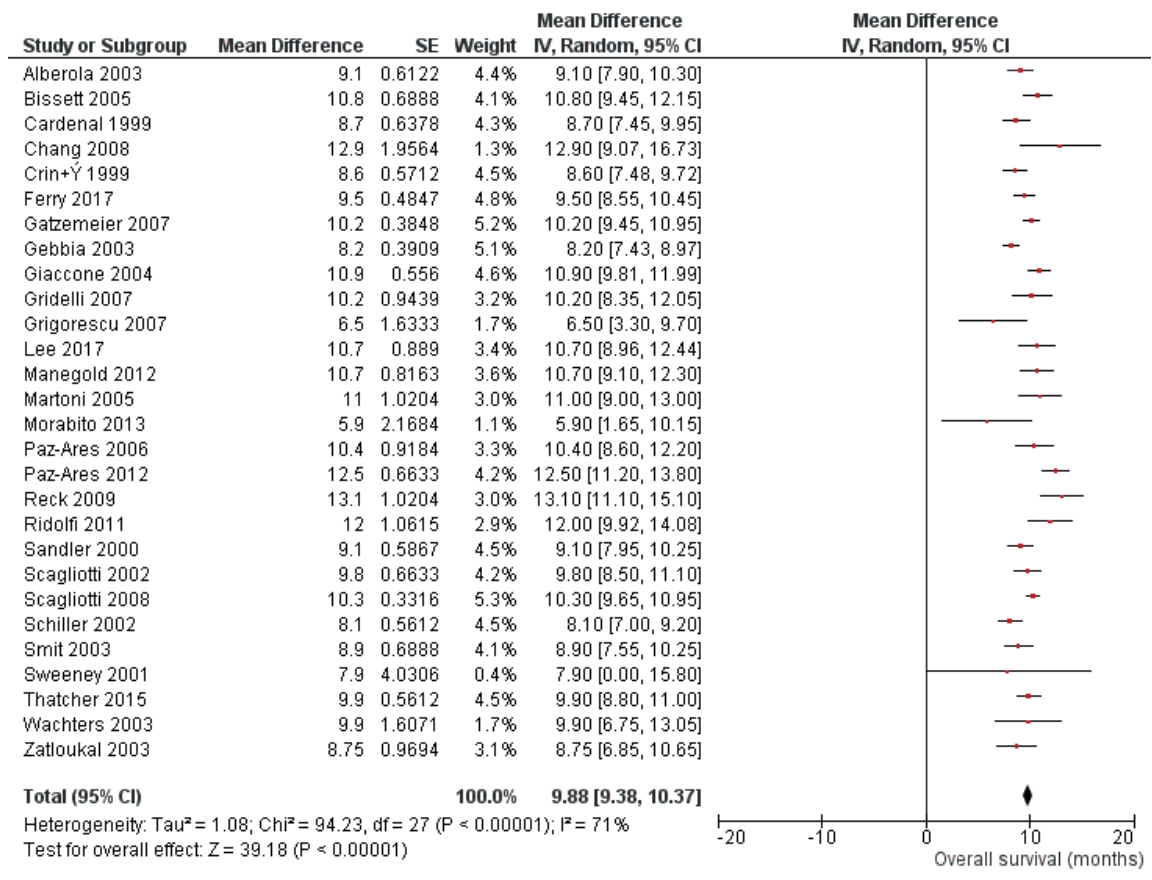

SFigure 4. Forest plot cisplatin-gemcitabine

STable 3. Clinical trial and real-world characteristics regimen cisplatin-gemcitabine

\begin{tabular}{lcc}
\hline Characteristic & Clinical trials & Real-world \\
\hline Total number of patients & 6736 & 214 \\
Overall survival (months) & 9.88 & 7.90 \\
Median age (years) & 61.0 & 63 \\
Male (\%) & 74.7 & 59 \\
Stage IV (\%) & 72.1 & 100 \\
ECOG Performance Status & & \\
ECOG PS 0-1 (\%) & 86.0 & 86 \\
ECOG PS $\geq 2(\%)$ & 13.7 & 6 \\
Missing & -- & 8 \\
Histology & & 48 \\
$\quad$ squamous (\%) & 36.6 & 32 \\
$\quad$ adenocarcinoma (\%) & 43.3 & 8 \\
large-cell (\%) & 7.7 & 12 \\
$\quad$ Other or unknown histology (\%) & 12.3 & \\
\hline
\end{tabular}

aECOG based on 26 references; Histology based on 25 references 


\section{Carboplatin pemetrexed}

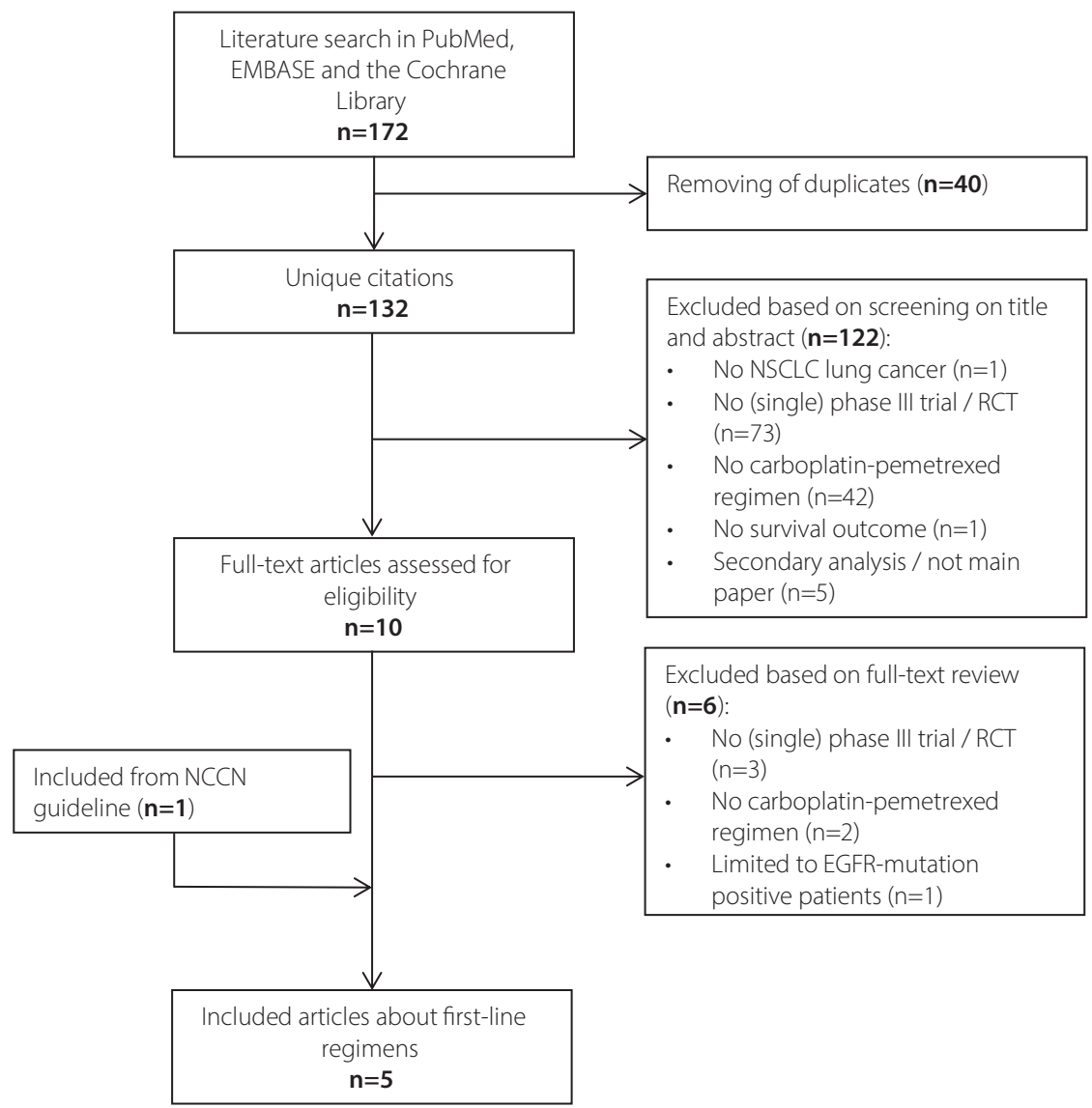

SFigure 5. Flowchart carboplatin-pemetrexed 


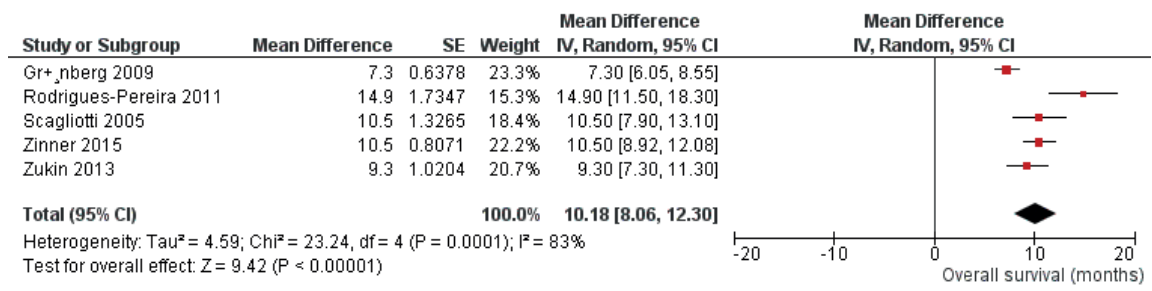

SFigure 6. Forest plot carboplatin-pemetrexed

STable 4. Clinical trial and real-world characteristics regimen carboplatin-pemetrexed

\begin{tabular}{lcc}
\hline Characteristic & Clinical trials & Real-world \\
\hline Total number of patients & 649 & 213 \\
Overall survival (months) & 10.18 & 6.51 \\
Median age (years) & 64.0 & 65 \\
Male (\%) & 63.9 & 62 \\
Stage IV (\%) & 82.0 & 100 \\
ECOG Performance Status & & \\
ECOG PS 0-1 (\%) & 72.8 & 81 \\
ECOG PS $\geq 2(\%)$ & 27.2 & 16 \\
Missing & -- & 3 \\
Histology & & 1 \\
$\quad$ squamous (\%) & 11.9 & 83 \\
adenocarcinoma (\%) & 68.4 & 9 \\
large-cell (\%) & 3.6 & 8 \\
\hline Other or unknown histology (\%) & 13.5 & \\
\hline
\end{tabular}




\section{Carboplatin gemcitabine}

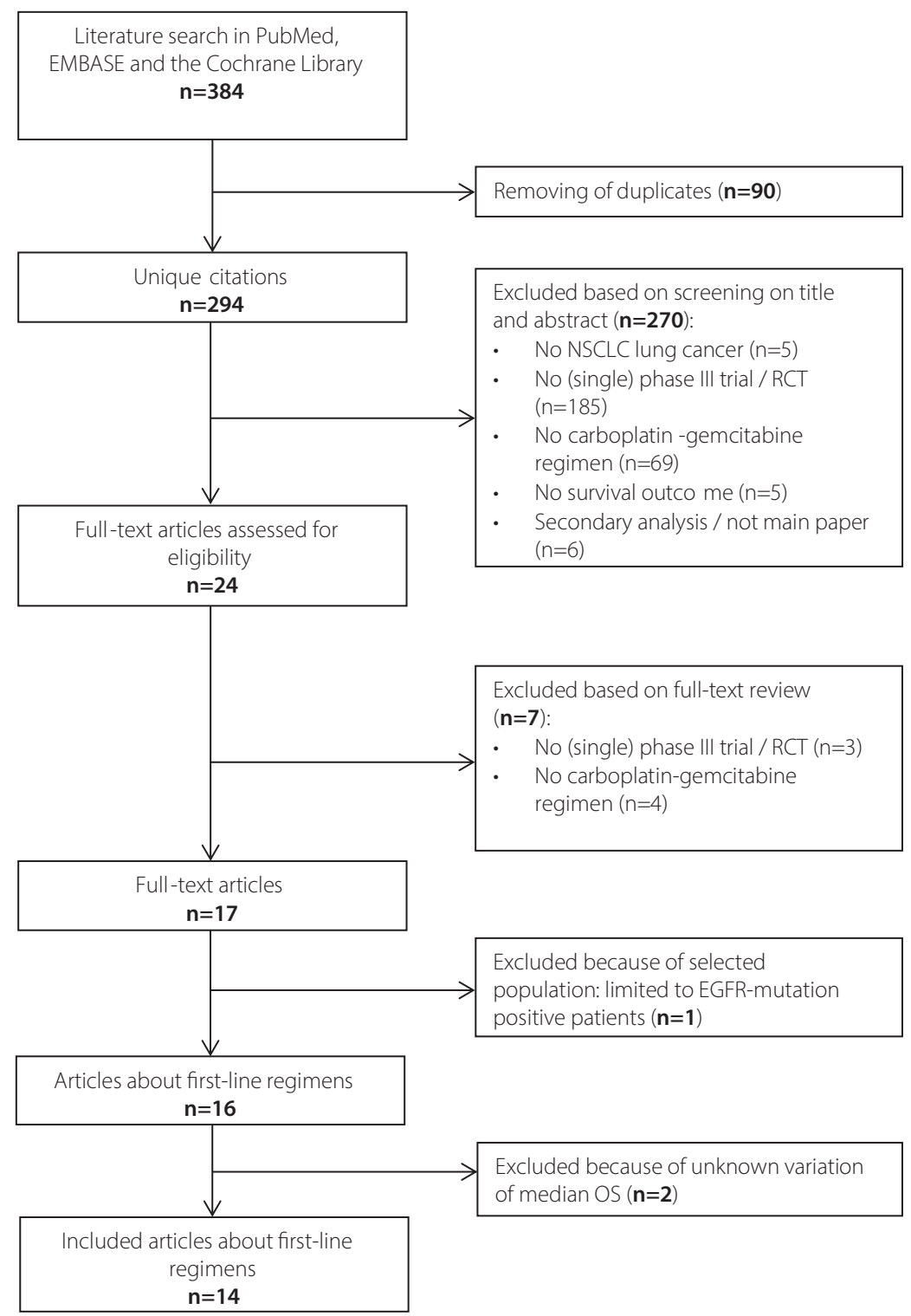

SFigure 7. Flowchart carboplatin-gemcitabine 


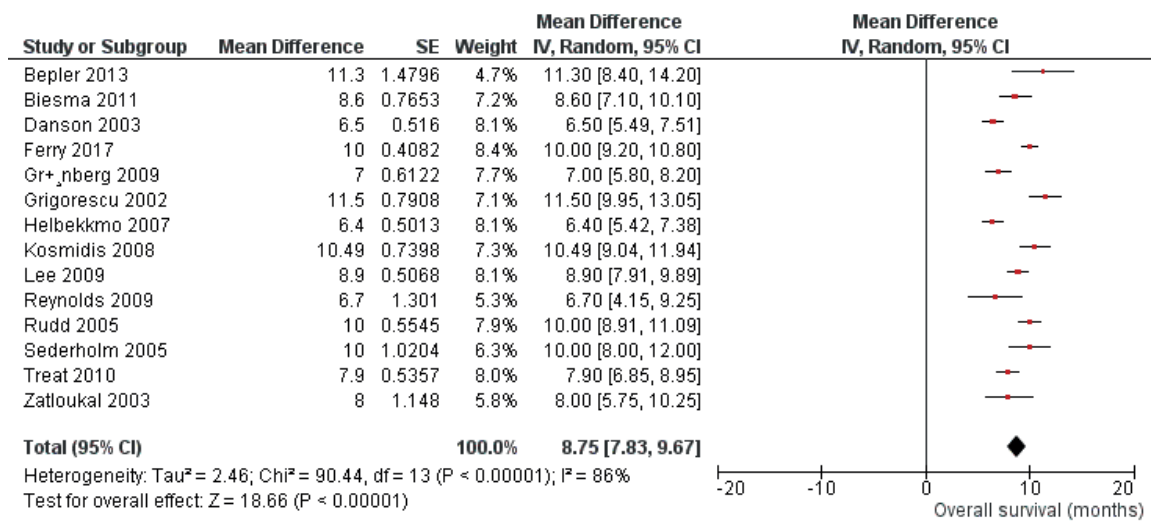

SFigure 8. Forest plot carboplatin-gemcitabine

STable 5. Clinical trial and real-world characteristics regimen carboplatin-gemcitabine

\begin{tabular}{lcc}
\hline Characteristic & Clinical trials & Real-world \\
\hline Total number of patients & 2857 & 171 \\
Overall survival (months) & 8.75 & 6.67 \\
Median age (years) & 63.1 & 68 \\
Male (\%) & 65.2 & 72 \\
Stage IV (\%) & 67.9 & 100 \\
ECOG Performance Status & & \\
ECOG PS 0-1 (\%) & 77.0 & 80 \\
ECOG PS $\geq 2$ (\%) & 22.9 & 16 \\
Missing & --- & 4 \\
Histology & & 40 \\
squamous (\%) & 29.7 & 30 \\
adenocarcinoma (\%) & 39.4 & 18 \\
large-cell (\%) & 6.1 & 12 \\
Other or unknown histology (\%) & 24.8 & \\
\hline
\end{tabular}




\section{Carboplatin paclitaxel bevacizumab}

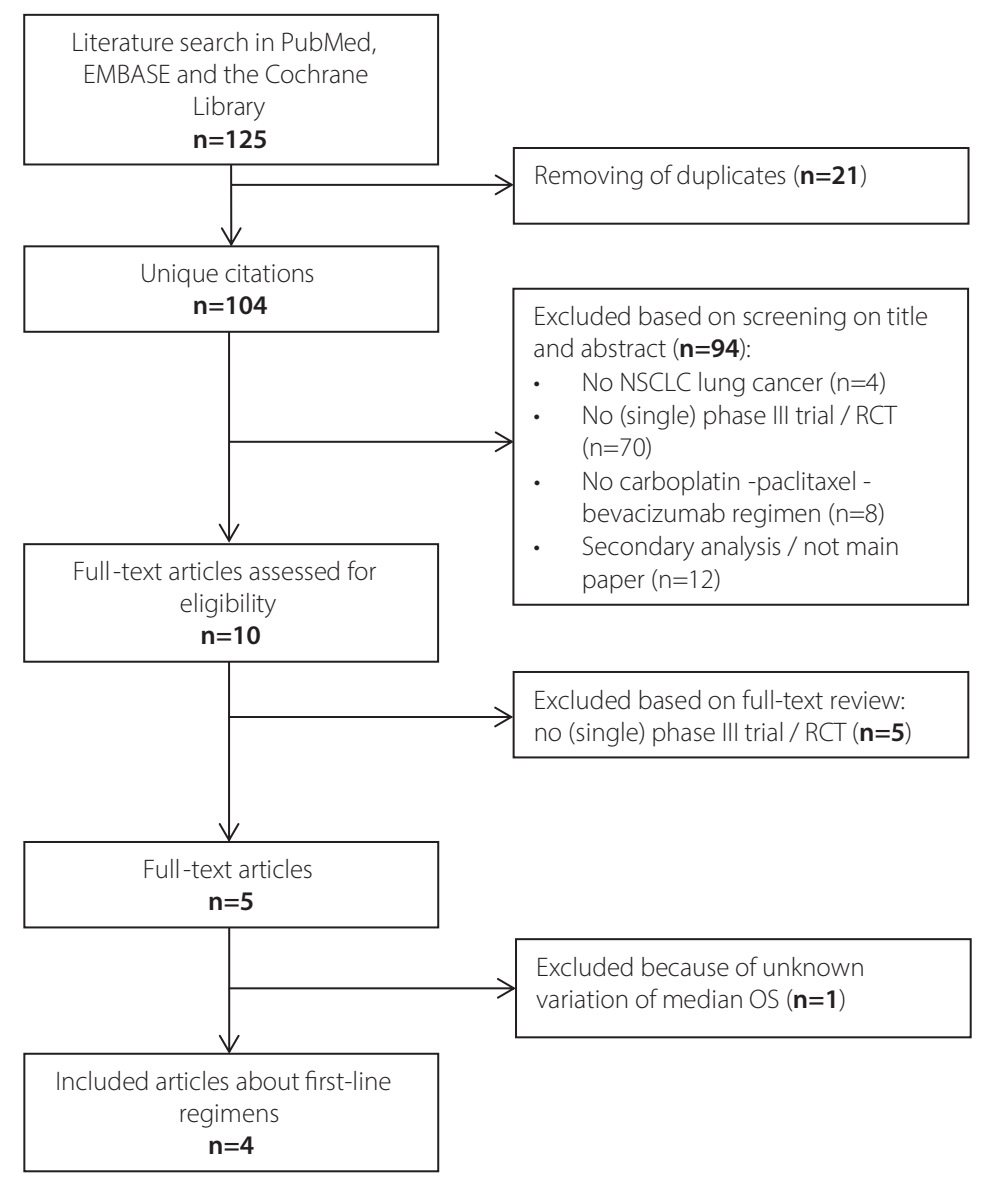

SFigure 9. Flowchart carboplatin-paclitaxel-bevacizumab 


\begin{tabular}{|c|c|c|c|c|c|c|c|}
\hline Study or Subgroup & Mean Difference & SE & Weight & $\begin{array}{l}\text { Mean Difference } \\
\text { IV, Fixed, } 95 \% \mathrm{Cl}\end{array}$ & & $\begin{array}{l}\text { Mean Difference } \\
\text { IV, Fixed, } 95 \% \mathrm{Cl}\end{array}$ & \\
\hline Galetta 2015 & 14.4 & 2.0918 & $3.7 \%$ & $14.40[10.30,18.50]$ & & & \\
\hline Patel 2013 & 13.4 & 0.7653 & $27.8 \%$ & $13.40[11.90,14.90]$ & & & $\rightarrow$ \\
\hline Sandler 2006 & 12.3 & 0.5731 & $49.6 \%$ & $12.30[11.18,13.42]$ & & & 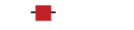 \\
\hline Zinner 2015 & 11.7 & 0.9301 & $18.8 \%$ & $11.70[9.88,13.52]$ & & & $\rightarrow$ \\
\hline Total $(95 \% \mathrm{Cl})$ & & & $100.0 \%$ & $12.57[11.78,13.36]$ & & & $\bullet$ \\
\hline $\begin{array}{l}\text { Heterogeneity: Chiz } \\
\text { Test for overall effec }\end{array}$ & $\begin{array}{l}3.04, d f=3(P=0.3 \\
Z=31.14(P<0.000\end{array}$ & $\begin{array}{l}\text { 9); }\left.\right|^{2}=19 \\
01)\end{array}$ & & & -10 & Overall s & $\begin{array}{cc}1 & 10 \\
\text { urvival (months) }\end{array}$ \\
\hline
\end{tabular}

SFigure 10. Forest plot carboplatin-paclitaxel-bevacizumab

STable 6. Clinical trial and real-world characteristics regimen carboplatin-paclitaxelbevacizumab

\begin{tabular}{lcc}
\hline Characteristic & Clinical trials & Real-world \\
\hline Total number of patients & 1121 & 73 \\
Overall survival (months) & 12.57 & 8.18 \\
Median age (years) & 64.9 & 62 \\
Male (\%) & 59.9 & 48 \\
Stage IV (\%) & 89.3 & 100 \\
ECOG Performance Status & & \\
ECOG PS 0-1 (\%) & 100 & 90 \\
ECOG PS $\geq 2$ (\%) & 0 & 7 \\
Missing & --- & 3 \\
Histology & & \\
$\quad$ squamous (\%) & 0.0 & 0 \\
adenocarcinoma (\%) & 85.7 & 10 \\
large-cell (\%) & 3.8 & 7 \\
other or unknown histology (\%) & 10.5 & \\
\end{tabular}




\section{Carboplatin docetaxel}

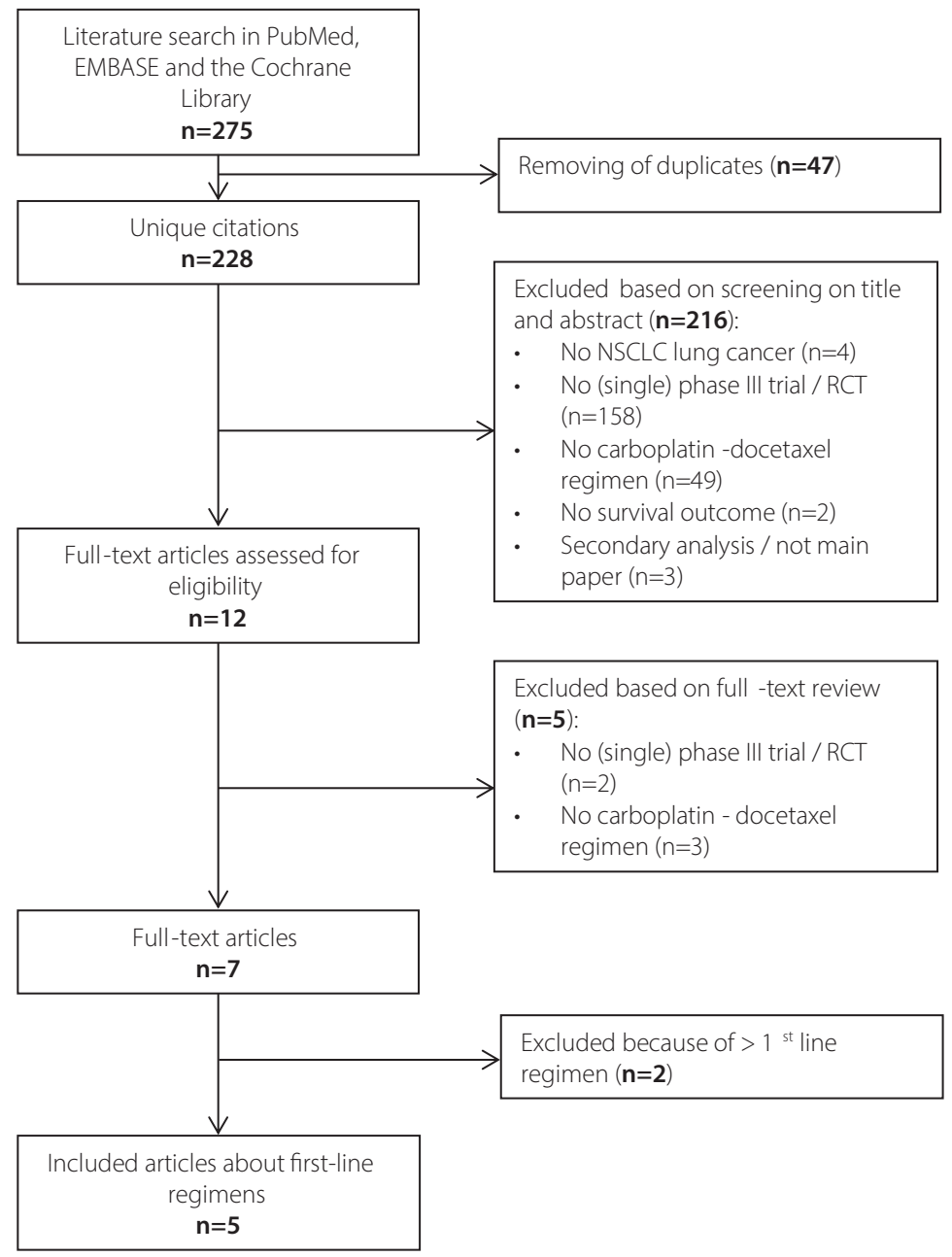

SFigure 11. Flowchart carboplatin-docetaxel 


\begin{tabular}{|c|c|c|c|c|c|c|c|}
\hline Study or Subgroup & Mean Difference & SE & Weight & $\begin{array}{l}\text { Mean Difference } \\
\text { IV, Random, } 95 \% \mathrm{Cl}\end{array}$ & \multicolumn{3}{|c|}{$\begin{array}{l}\text { Mean Difference } \\
\text { IV, Random, } 95 \% \mathrm{Cl}\end{array}$} \\
\hline Booton 2006 & 9.5 & 0.8163 & $20.2 \%$ & $9.50[7.90,11.10]$ & & & $=-$ \\
\hline Fossella 2003 & 9.4 & 0.4847 & $26.8 \%$ & $9.40[8.45,10.35]$ & & & - \\
\hline Groen 2011 & 8.2 & 0.3316 & $29.6 \%$ & $8.20[7.55,8.85]$ & & 를 & \\
\hline Rodrigues-Pereira 2011 & 14.7 & 2.2959 & $5.6 \%$ & $14.70[10.20,19.20]$ & & & \\
\hline Schuette 2011 & 10.3 & 0.954 & $17.8 \%$ & $10.30[8.43,12.17]$ & & & $\rightarrow$ \\
\hline Total $(95 \% \mathrm{Cl})$ & & & $100.0 \%$ & $9.52[8.35,10.69]$ & & & \\
\hline \multicolumn{5}{|c|}{$\begin{array}{l}\text { Heterogeneity: } \operatorname{Tau}^{2}=1.09 ; \mathrm{Ch}^{2}=14.57, \mathrm{df}=4(\mathrm{P}=0.006) ; \mathrm{I}^{2}=73 \% \\
\text { Test for overall effect: } Z=15.96(P<0.00001)\end{array}$} & $\mathfrak{b}_{-20}$ & $\begin{array}{lr}0 & 1 \\
\text { Overall sun }\end{array}$ & $10 \quad 20$ \\
\hline
\end{tabular}

SFigure 12. Forest plot carboplatin-docetaxel

STable 7. Clinical trial and real-world characteristics regimen carboplatin-docetaxel

\begin{tabular}{lcc}
\hline Characteristic & Clinical trials & Real-world \\
\hline Total number of patients & 1100 & 45 \\
Overall survival (months) & 9.52 & 4.93 \\
Median age (years) & 61.0 & 59 \\
Male (\%) & 66.6 & 51 \\
Stage IV (\%) & 72.0 & 100 \\
ECOG Performance Status & & \\
ECOG PS 0-1 (\%) & 87.5 & 89 \\
ECOG PS $\geq 2$ (\%) & 12.5 & 9 \\
Missing & --- & 2 \\
Histology & & \\
$\quad$ squamous (\%) & 27.1 & 9 \\
adenocarcinoma (\%) & 51.4 & 51 \\
large-cell (\%) & 10.5 & 20 \\
Other or unknown histology (\%) & 11.0 & 20 \\
\hline
\end{tabular}

aECOG based on 3 references 


\section{Gefitinib (in EGFR-mutation positive patients)}

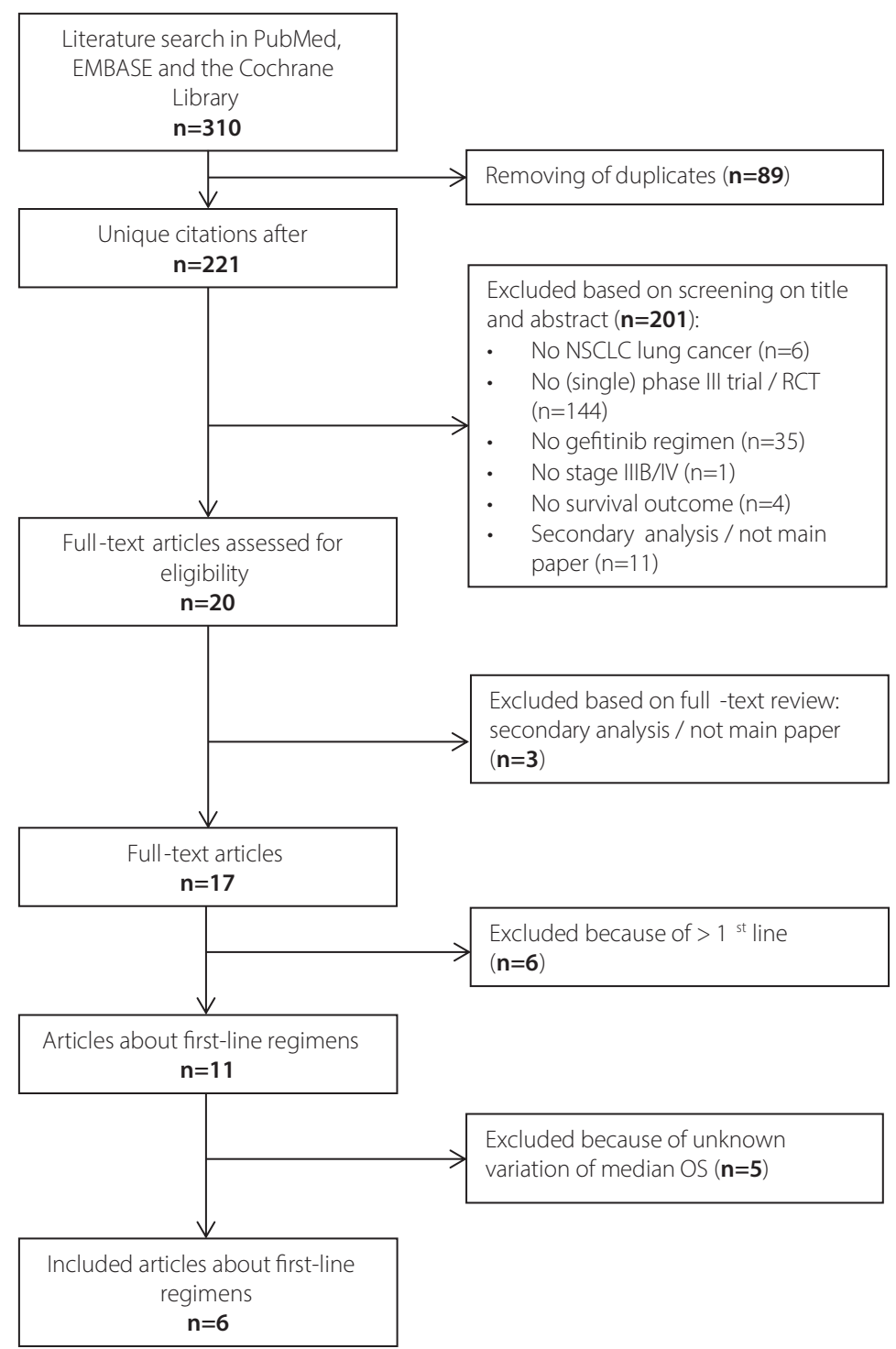

SFigure 13. Flowchart gefitinib 


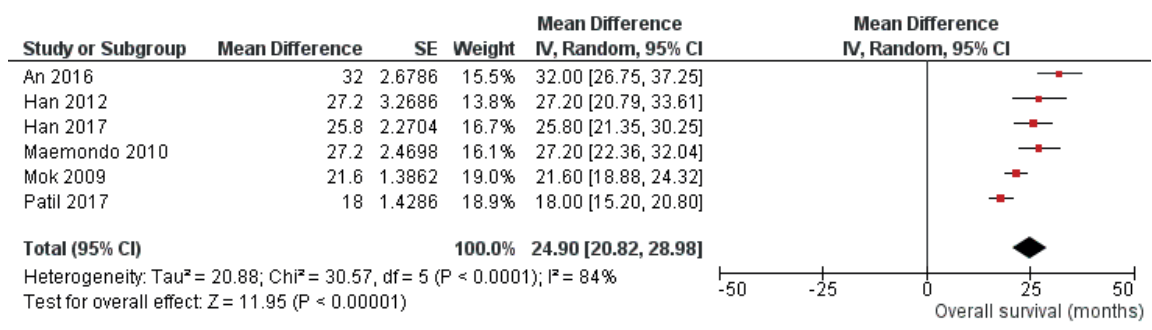

SFigure 14. Forest plot gefitinib

STable 8. Clinical trial and real-world characteristics regimen gefitinib

\begin{tabular}{lcc}
\hline Characteristic & Clinical trials & Real-world \\
\hline Total number of patients & 503 & 35 \\
Overall survival (months) & 24.90 & 21.19 \\
Median age (years) & 63.9 & 68 \\
Male (\%) & 45.6 & 43 \\
Stage IV (\%) & 87.6 & 100 \\
ECOG Performance Status & & \\
ECOG PS 0-1 (\%) & 97.4 & 89 \\
ECOG PS $\geq 2(\%)$ & 2.6 & 11 \\
Missing & --- & 0 \\
Histology & & 0 \\
squamous (\%) & 0.4 & 91 \\
adenocarcinoma (\%) & 98.2 & 0 \\
large-cell (\%) & 0.2 & 9 \\
other or unknown histology (\%) & 1.1 & \\
\hline
\end{tabular}

aNote: the characteristics from 2 references are based on the whole patient group, not only on the EGFR+ group; ECOG PS based on 3 references 


\section{Erlotinib (in EGFR-mutation positive patients)}

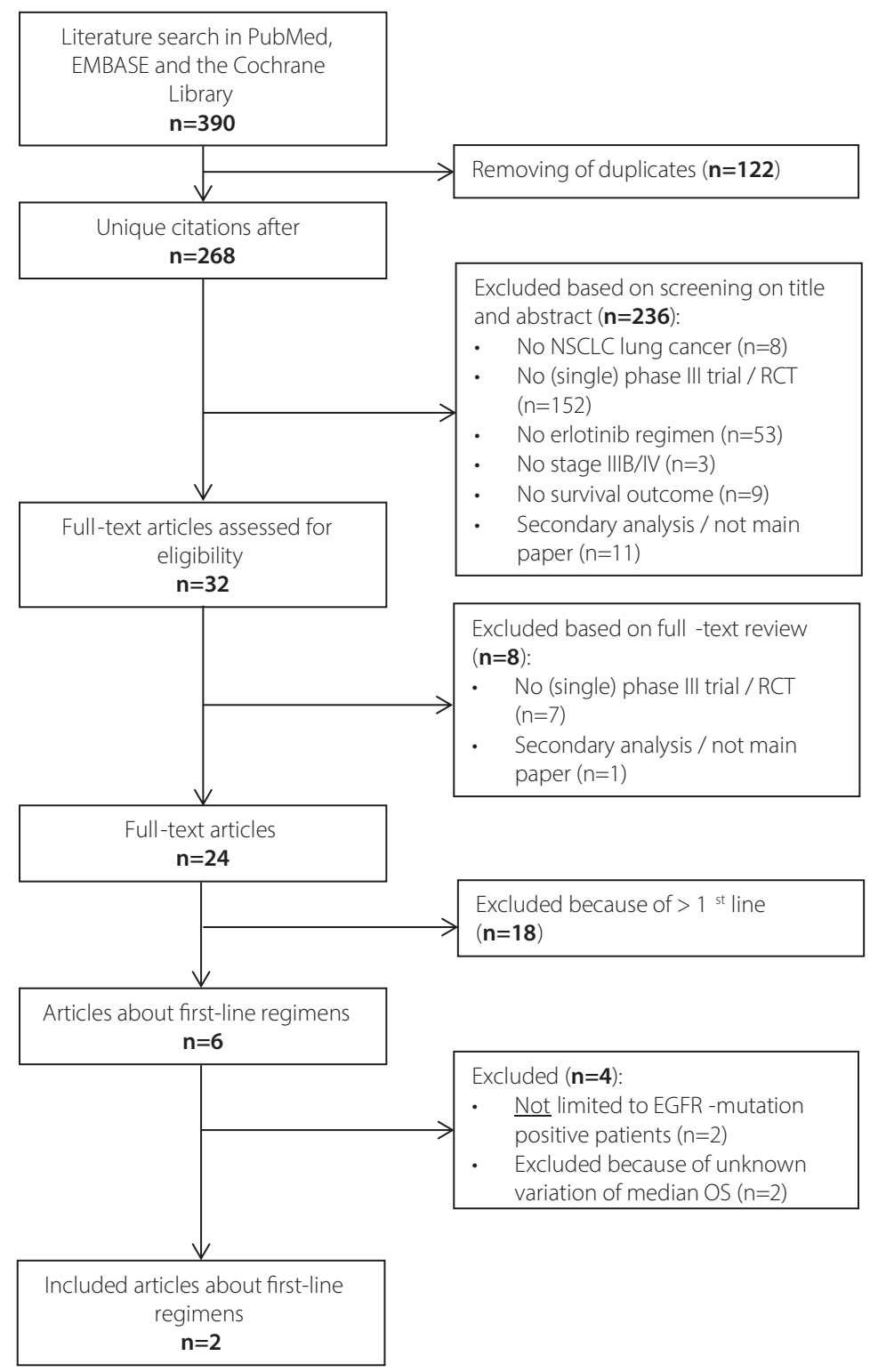

SFigure 15. Flowchart erlotinib 


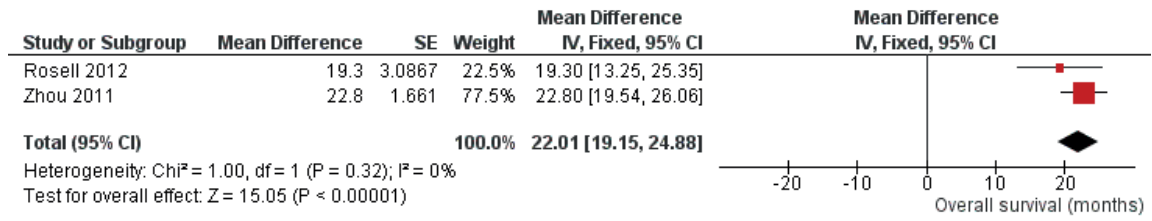

SFigure 16. Forest plot erlotinib

STable 9. Clinical trial and real-world characteristics regimen erlotinib

\begin{tabular}{lcc}
\hline Characteristic & Clinical trials & Real-world \\
\hline Total number of patients & 168 & 24 \\
Overall survival (months) & 22.01 & 14.32 \\
Median age (years) & 61.0 & 68 \\
Male (\%) & 37.0 & 25 \\
Stage IV (\%) & 89.0 & 100 \\
ECOG Performance Status & & \\
ECOG PS 0-1 (\%) & 88.5 & 75 \\
ECOG PS $\geq 2$ (\%) & 11.5 & 17 \\
Missing & --- & 8 \\
Histology & & 4 \\
squamous (\%) & 1.0 & 79 \\
adenocarcinoma (\%) & 95.0 & 0 \\
large-cell (\%) & 3.0 & 17 \\
Other or unknown histology (\%) & 0.0 & \\
\hline
\end{tabular}

aHistology based on 1 reference 


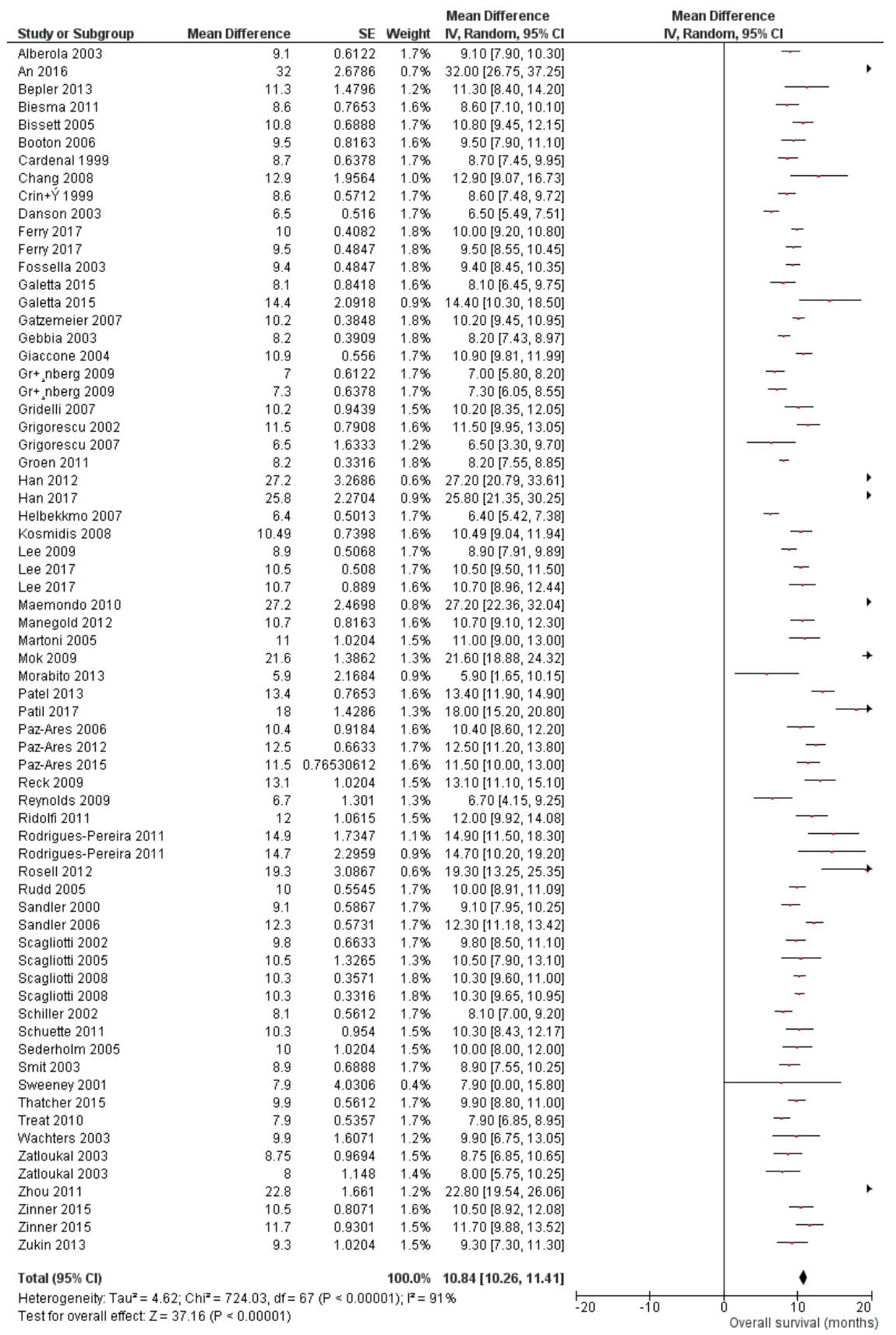

SFigure 17. Forest plot with overview of clinical trials for reference outcome first-line chemotherapy 


\section{APPENDIX 3 PROXIES FOR TOXICITY}

STable 10. Dose reduction and switches to other systemic agents as proxies for toxicity

\begin{tabular}{|c|c|c|}
\hline & $\begin{array}{c}\text { Dose reduction }^{a} \\
n(\%)\end{array}$ & $\begin{array}{c}\text { Switch to other } \\
\text { systemic agents } \\
n(\%)\end{array}$ \\
\hline Cisplatin pemetrexed & $44(13)$ & 65 (19) \\
\hline Cisplatin gemcitabine & $41(19)$ & $50(23)$ \\
\hline Carboplatin pemetrexed & $38(18)$ & $2(1)$ \\
\hline Carboplatin gemcitabine & $66(39)$ & $4(2)$ \\
\hline Bevacizumab carboplatin paclitaxel & $12(16)$ & $7(10)$ \\
\hline Carboplatin docetaxel & $5(11)$ & $2(4)$ \\
\hline Gefitinib (in EGFR positive patients) c & 0 & 0 \\
\hline Erlotinib (in EGFR positive patients) & $3(13)$ & $1(4)$ \\
\hline Overall & 209 (19) & $131(12)$ \\
\hline
\end{tabular}

aMissing values in 39 patients (3\%)

bMissing values in 34 patients (3\%)

cPossible underestimation as data about gefitinib from earlier years was not available in the databases

STable 11. Co-morbidities in patients with early discontinuation of systemic therapy

\begin{tabular}{lccc}
\hline Co-morbidity & \multicolumn{2}{c}{ Early discontinuation } & p-value \\
& Yes $(\mathbf{n = 4 6 9 )}$ & No $(\mathbf{n}=653)$ & \\
C (\%) & n (\%) & \\
COPD & $92(20)$ & $108(17)$ & 0.206 \\
coronary artery disease & $64(14)$ & $75(12)$ & 0.312 \\
diabetes & $54(12)$ & $60(9)$ & 0.229 \\
peripheral vascular disease & $52(11)$ & $64(10)$ & 0.488 \\
other cancer & $29(6)$ & $39(6)$ & 0.900 \\
cerebrovascular disease & $35(8)$ & $28(4)$ & 0.025 \\
congestive heart failure & $18(4)$ & $16(3)$ & 0.217 \\
connective tissue disease & $11(2)$ & $11(2)$ & 0.514 \\
\hline
\end{tabular}




\section{APPENDIX 4 LIST OF INCLUDED HOSPITALS}

The individual Santeon hospitals include:

- Canisius Wilhelmina Hospital, Nijmegen, the Netherlands

- Catharina Hospital, Eindhoven, the Netherlands

- Martini Hospital, Groningen, the Netherlands

- Medisch Spectrum Twente, Enschede, the Netherlands

- OLVG, Amsterdam, the Netherlands

- St. Antonius Hospital, Nieuwegein/Utrecht, the Netherlands

- Maasstad Hospital, Rotterdam, the Netherlands (member since 2017, not included in this study)

www.santeon.nl 
EE GAP IN METASTATIC NSCLC | 103 



\section{Chapter 4}

Trends in drug costs and overall survival in patients with metastatic non-small cell lung cancer in the Netherlands diagnosed from 2008 through 2014

Christine M. Cramer-van der Welle, Bas J.M. Peters, Maarten J. Deenen, Franz M.N.H. Schramel, Ewoudt M.W. van de Garde 


\section{ABSTRACT}

Objectives: The Value-Based Health Care concept defines patient value as patient-relevant outcomes divided by costs. The aim of the present study was to assess the development of systemic treatment costs over the years compared with changes in overall survival (OS) at the level of a diagnosis of stage IV non-small cell lung cancer (NSCLC).

Methods: All patients diagnosed (in 2008-2014) with stage IV NSCLC and treated with systemic treatment in six Dutch large teaching hospitals (Santeon network) were included. We collected data on OS and amounts of drug units (milligrams) for every drug in the applied systemic cancer treatments, until death. These amounts were multiplied by Dutch unit costs (Euros/mg) expressed in 2018 Euros to construct total drug costs per line of treatment per patient. Costs for day care visits were added for drugs requiring parenteral administration.

Results: Data were collected from 1214 patients. Median OS and mean total drug costs showed no significant variation over the years ( $p=0.437$ and $p=0.693$, respectively). Mean total drug costs per 1 year of survival ranged from $€ 20,665$ to $€ 26,438$ during the period under study. Costs for first-line systemic treatment were significantly higher in 2011-2014 compared with 2008-2010.

Conclusion: This study shows that overall drug costs were stable over the years, despite a relative increase in first-line treatment costs. Median OS remained at around 8 months from year to year. These trend data are very relevant as background for the assessment of costs and achieved outcomes in the more recent years. 


\section{INTRODUCTION}

Non-small cell lung cancer (NSCLC) comprises 73\% of lung cancer diagnoses in the Netherlands and is usually detected after the occurrence of regional or distant metastases. The overall 1-year survival rate of metastatic NSCLC (stage IV) in the Netherlands is only 23\% [1]. Treatment options for lung cancer have expanded in recent years with the arrival of targeted therapies and immunotherapies. Targeted agents for patients with epidermal growth factor receptor (EGFR) mutations and anaplastic lymphoma kinase (ALK) rearrangements have proved benefits in a small number of patients with NSCLC. More recently, immunotherapeutic agents directed on programmed cell death-1 receptor (PD-1) or its ligand PD-L1 have demonstrated a broader benefit and durable responses in NSCLC [2]. However, concern is growing that the magnitude of the survival benefit from these new anticancer therapies does not justify its high prices [3-6]. Moreover, the benefits of new treatments might not be clinically meaningful to patients [7]. Kemp and Prasad stated that in many cases there is insufficient evidence to reliably make the assertion that treatments that fail to improve overall survival (OS), but do improve surrogate outcomes, may still have meaningful clinical benefit [8].

Although traditional single intervention cost-effectiveness studies based on clinical trial data are widely available $[9,10]$, observational data about the actual costs and OS outcomes associated with systemic treatment regimens for NSCLC in real-world are still scarce. Recently published research on treatment costs for stage IV NSCLC in real-world practice showed that these costs were substantial, despite the fact that median OS benefit was poor [11]; however, follow-up time was limited and results over the years were lacking. In particular, trend data covering an extended period will be important as a reflection on the development of the costs of drugs and corresponding established outcomes informing future payer/policy decision-making.

Recently, we have constructed a large real-world cohort with highly detailed data about systemic treatments and outcomes for patients diagnosed with stage IV NSCLC in the Netherlands in 2008-2014 [12]. The aim of the present study was to explore trends in systemic treatment costs for all patients with metastasized NSCLC and the corresponding OS achieved in consecutive years in this cohort. 


\section{METHODS}

\section{Study population and drug use}

All newly diagnosed patients with stage IV NSCLC (in 2008-2014) who were treated with systemic anticancer treatment in six Dutch large teaching hospitals (Santeon network) were captured from the previously described cohort [12]. For each patient, we extracted type of drug, dose, date of administration, and administration route of all anti-cancer drugs, from the date of diagnosis until death, from the Santeon Farmadatabase (SFD), which includes detailed data about prescribed pharmacotherapy [13]. When necessary, additional data on systemic treatments was derived from individual patient files. Next, for parenteral drugs, the sum of all actual doses given was calculated per regimen for each individual patient. For oral drugs, the dispensed amount of units or end date and frequency were used to calculate the total numer of units applied. In case of missing values in patients receiving parenteral drugs, these were imputed with mean values from patients with the same treatment. For oral drugs, we used standard doses (e.g. erlotinib $150 \mathrm{mg}$, gefitinib $250 \mathrm{mg}$ ) and mean number of treatment days per regimen in case of missing values.

\section{Patient outcomes}

OS was calculated based on the time between the date of treatment start and the date of death (as derived from the Personal Records Database (BRP) of the Government of the Netherlands). Patients still alive at 31 January 2017 ( $n=59 ; 5 \%)$ were censored. Four of these patients were receiving active treatment at that time.

\section{Drug costs}

Pharmacy purchase prices were extracted from the official price list that is published by Z-index.nl BV [14] in April in the respective years, including 6\% VAT (Appendix Table 2). In case of more than one manufacturer, the lowest price per milligram was selected. Subsequently, the pharmacy purchase prices have been adjusted for inflation to reflect the 2018 price level (2018 Euros) using the general price index as published by Statistics Netherlands [15]. Costs per patients were calculated by multiplying the number of drugs applied (milligrams) by cost/milligram, expressed in Euros corresponding with the calendar years of drug treatment, for every drug in the applied systemic cancer treatments, until death.

In case of parenterally administered drugs, the costs for a day care visit were added per unique date of administration. Costs for day care visits were obtained from the Dutch manual for costing studies [16-18] and have been adjusted for inflation towards 2018 [15]. 


\section{Statistics}

Descriptive statistics were used to describe survival and costs. Costs were expressed as mean \pm standard deviation (SD), and OS as median (with 95\% confidence interval), as obtained from a Kaplan-Meier survival analysis. Overall mean costs per year of diagnosis were calculated by dividing the total costs from patients with that same year of diagnosis by total number of patients in that same year of diagnosis who started systemic treatment. Subsequently, overall mean costs were subdivided into mean costs per respective line of treatment to illustrate the relative contributions thereof to the total drug costs of illness over the years. To do so, the costs from that line of treatment were again divided by the total number of patients in that same year of diagnosis who started any systemic treatment. In addition, the absolute mean costs per line of treatment have been calculated for the entire cohort based on patients who actually received the corresponding line of treatment. The analysis covered three parts: 1) variation in costs over the years; 2) variation in survival over the years; and 3) variation in the ratio between costs and median survival over the years. One-way analysis of variance (ANOVA) was applied to assess statistically significant differences in costs, and the log-rank test was applied to test for differences in survival. All statistical analyses were performed in IBM SPSS Statistics 24 (IBM Corporation, Armonk, NY, USA). A p-value $<0.05$ was considered statistically significant.

\section{RESULTS}

Data were collected from 1214 patients with stage IV NSCLC and systemic treatment (see Table 1 for patient characteristics). The vast majority of patients (>90\%) received a platinum-doublet or an EGFR inhibitor as first-line treatment (see Cramer et al. [12] for administration frequencies). Of these patients, 34\% received second-line systemic treatment and $8 \%$ received further line(s) of treatment. The most prevalent second-line treatments were docetaxel (29\%), pemetrexed (20\%), and erlotinib (19\%). Figure 1 shows the drug costs per patient (mean $\pm \mathrm{SD}$ ), survival curves, and ratio between costs and median survival categorized by year of diagnosis. Imputation of missing values was applied in 62 patients (5\%). The mean costs for first-line treatment, second-line treatment, and overall were $€ 12,209$, $€ 3043$, and $€ 16,116$, respectively.

Mean total drug costs (including parenteral administration) per 1 year of survival ranged from $€ 20,665$ to $€ 26,438$ during the period under study. No significant difference was found in overall mean costs over the years $(p=0.693)$. Costs for first-line systemic treatment were significantly higher in 2011-2014 compared with 2008-2010 ( $p=0.005)$ (pre and post introduction of maintenance therapy). Median OS remained at approximately the same value from year to year, with a range from 7.3 to 8.7 months (log-rank test $p=0.437$ ). 


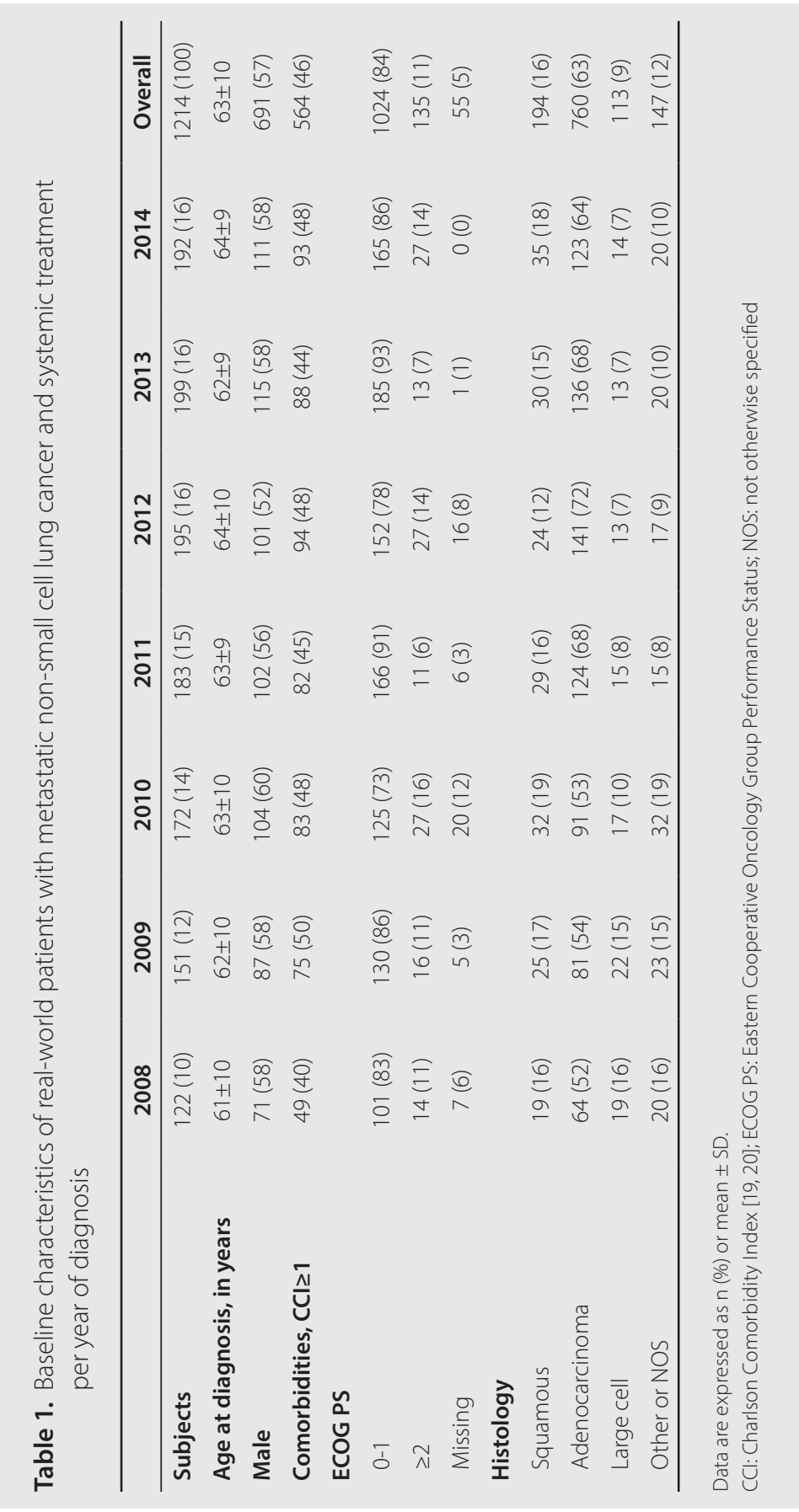



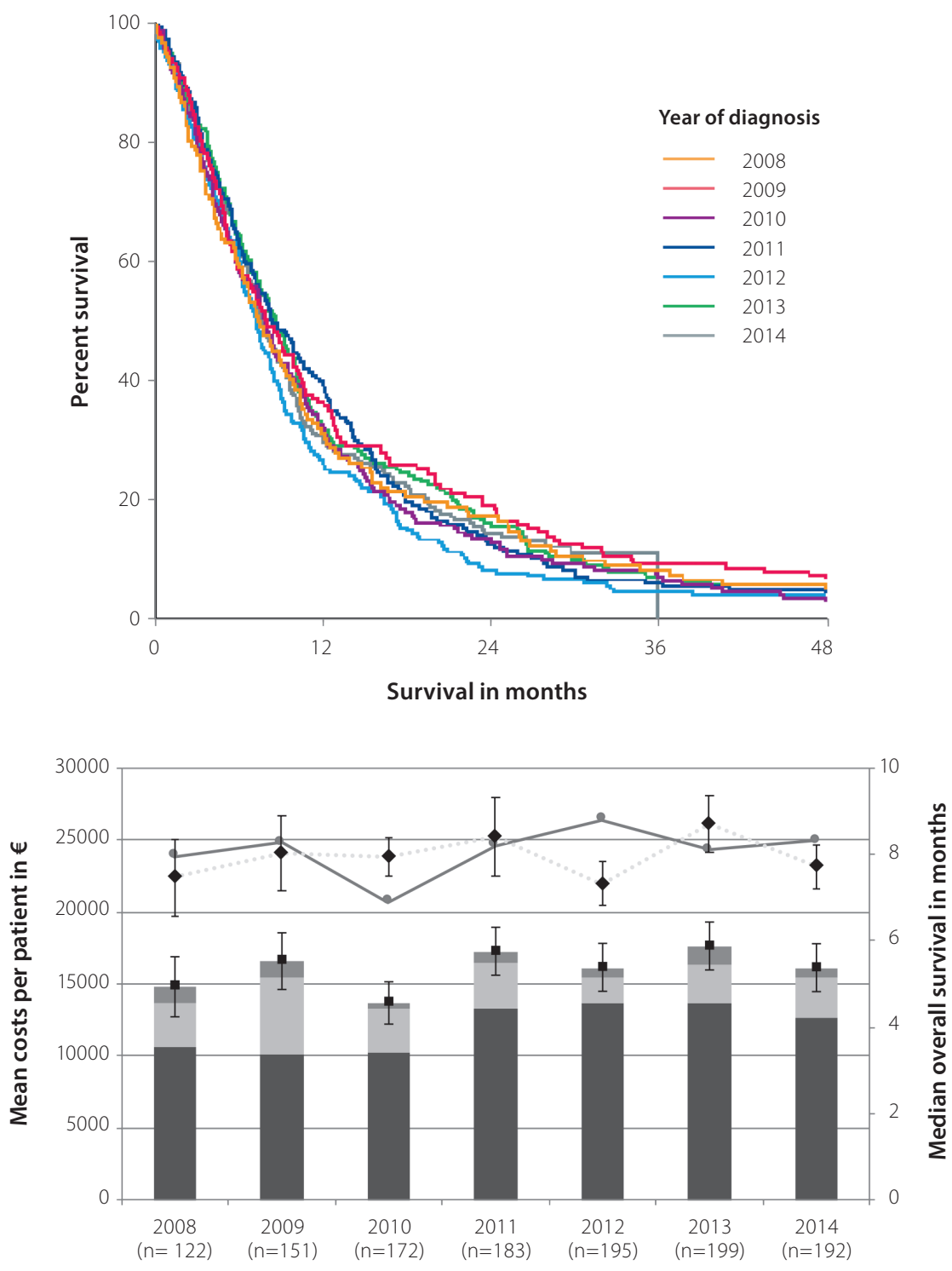

Year of diagnosis

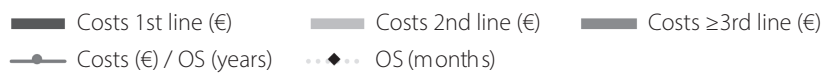

Figure 1. Costs and survival in real-world patients with metastatic non-small cell lung cancer and systemic treatment diagnosed from 2008 through 2014. OS: overall survival 


\section{DISCUSSION}

This study showed no significant alteration in total drug costs and OS for patients with stage IV NSCLC over the years; however, costs for first-line systemic treatment were higher in 2011-2014 compared with 2008-2010. The likely explanation for this increase in first-line costs is the introduction of pemetrexed maintenance in 2011 in The Netherlands after the publication of a randomized phase III trial of maintenance pemetrexed versus placebo by Ciuleanu et al. [21].

To the best of our knowledge, the data presented are the only data on drug costs and the relation with OS in metastatic NSCLC in consecutive years in The Netherlands.

Overall, the results regarding mean costs found in our study are in line with data from another study from The Netherlands. Van der Linden et al. reported total mean drug costs of €4970 for stage IV patients with first-line chemotherapy or targeted therapy, including costs for day care [22]. Considering that they studied patients irrespective of treatment (thus also including patients receiving supportive care only), and assuming that $55 \%$ of diagnosed patients receives supportive care only $[12,23]$, this resembles total mean costs for first-line systemic treatment of $€ 11,044$, which is quite similar to our finding of mean drug costs of $€ 12,209$ for first-line systemic treatment. In contrast, our results differ substantially from the costs found by Keusters et al. [24]. This cost-of-illness study reported mean total costs of $€ 9427$ for oncolytic drugs and day care (the sum of $€ 6390 / 0.761$, and $€ 615 / 0.597$, respectively, when accounting for percentage of treated patients), which is much lower than our finding of mean total drug costs of $€ 16,116$. However, their research was performed in a tertiary cancer centre and excluded all patients that were eligible for an ongoing clinical trial, which might have led to selection bias towards patients with poorer prognosis and earlier discontinuation of systemic treatment, and consequently less costs. Besides this, Keusters et al. showed that the average cost for metastatic NSCLC over time in The Netherlands has decreased (in 2006-2012 compared with 2003-2005), while expenses for oncolytic drugs rose. They expect that the trend of decreasing costs over time will not be representative, as immunomodulatory drugs will drive the trend in the opposite direction [12].

A strength of this study is the large and unselected population of real-world patients with detailed data regarding systemic treatments and actual doses for each individual patient. This means, for example, that early discontinuations and dose reductions are accounted for. Furthermore, by using historical drug list prices adjusted for inflation, our study reflects true alterations in treatment planning and incorporates price drops after expiration of patents, if any. Finally, this study is the first to report changes in systemic treatment costs over the years compared with changes in survival prognosis at the level of a diagnosis of stage IV NSCLC. The latter in contrast with a traditional health technology assessment, which are focused on the cost-effectiveness of one or more unique interventions for these patients. 
A limitation of this study could be our restriction to direct drug costs only. For example, we excluded other medical costs for lung cancer patients, such as outpatient visits, laboratory tests, visits to other healthcare providers, and treatments for adverse events. As a consequence, if newer, more expensive drugs cause less toxicity (thus saving costs for treatment of toxicity), this is not accounted for in our study. However, direct drug costs will give valuable insights when comparing standard chemotherapy with chemotherapy plus immunotherapy as a novel treatment option [25-27] in the assessment of costs and outcomes in more recent years. By adding immunotherapy to chemotherapy, net drug costs will increase while other costs and the toxicity profile remain more or less the same $[28,29]$.

Second, patients still alive at database closure could potentially have received further treatment ( $n=4$ were still receiving active treatment and could possibly start a new line of treatment in the future), of which the costs are not captured in this study, thus resulting in an underestimation of mean total costs. Another reason for underestimation of costs could be that patients moved to another site of care after completion of treatment, with the risk of unknown subsequent lines of treatment before death. However, due to the small number of patients still alive $(<5 \%)$ and the virtually complete first-line costs for these patients, which account for the largest part of total mean costs, those limitations will only cause a small underestimation.

Third, one could argue about changes in OS as a sole measure for benefit from systemic treatment. Measures on quality of life (QoL) could be informative as well to assess the benefit from systemic treatments over the years. However, OS remains an objective outcome since it represents survival time, a direct and unambiguous patient benefit. Furthermore, several challenges in measuring QoL exist regarding patient follow-up in the palliative phase.

Finally, a limitation could be that novel treatment options such as immunotherapy were not covered by the time frame under study. Notwithstanding, this study provides insights into the costs for systemic treatments in the pre-immunotherapy period and could serve as an important reference point to assess the economic impact of immunotherapy.

In a few years' time, a follow-up study can show how the costs and survival benefit ratio continues. Considering patient value as seen with the Value-Based Health Care concept [30], the ratio between outcomes and costs should increase. When studying this, it is important to consider that many countries have confidential discounts on newer biological therapies nowadays. In the end, the development of drug costs per patient with metastatic NSCLC will be the result of the interplay between new (more) expensive and effective treatments coming to market and potential price drops linked to the introduction of generics and biosimilars [31]. 


\section{CONCLUSIONS}

Overall costs for systemic drug treatment in patients with metastatic NSCLC were stable over the years 2008-2014, despite a relative increase in first-line treatment costs from 2011 onwards. Furthermore, OS in the corresponding years showed no improvements so far. These trend data are very relevant as background for the assessment of costs and achieved outcomes in more recent years, with newer treatment options available. Following the Value-Based Health Care theory, two times more/better outcomes would allow, at maximum, a doubling of the costs of delivering these outcomes to keep value at the status quo. 


\section{REFERENCES}

1. The Netherlands Cancer Registry. www.cijfersoverkanker.nl. Accessed Nov 13, 2018.

2. E.C. Naylor, J.K. Desani, P.K. Chung, Targeted Therapy and Immunotherapy for Lung Cancer, Surg Oncol Clin N Am 25(3) (2016) 601-9.

3. J.C. Del Paggio, R. Sullivan, D. Schrag, W.M. Hopman, B. Azariah, C.S. Pramesh, I.F. Tannock, C.M. Booth, Delivery of meaningful cancer care: a retrospective cohort study assessing cost and benefit with the ASCO and ESMO frameworks, Lancet Oncol 18(7) (2017) 887-894.

4. D. Cohen, Cancer drugs: high price, uncertain value, BMJ 359 (2017) j4543.

5. D.M. Jiang, K.K.W. Chan, R.W. Jang, C. Booth, G. Liu, E. Amir, R. Mason, L. Everest, E. Elimova, Anticancer drugs approved by the Food and Drug Administration for gastrointestinal malignancies: Clinical benefit and price considerations, Cancer Med 8(4) (2019) 1584-1593.

6. H.M. Kantarjian, T. Fojo, M. Mathisen, L.A. Zwelling, Cancer drugs in the United States: Justum Pretium--the just price, J Clin Oncol 31(28) (2013) 3600-4.

7. C. Davis, H. Naci, E. Gurpinar, E. Poplavska, A. Pinto, A. Aggarwal, Availability of evidence of benefits on overall survival and quality of life of cancer drugs approved by European Medicines Agency: retrospective cohort study of drug approvals 2009-13, BMJ 359 (2017) j4530

8. R. Kemp, V. Prasad, Surrogate endpoints in oncology: when are they acceptable for regulatory and clinical decisions, and are they currently overused?, BMC Med 15(1) (2017) 134.

9. M.L. Bongers, V.M. Coupe, E.P. Jansma, E.F. Smit, C.A. Uyl-de Groot, Cost effectiveness of treatment with new agents in advanced non-small-cell lung cancer: a systematic review, Pharmacoeconomics 30(1) (2012) 17-34.

10. C.T.T. Nguyen, F. Petrelli, S. Scuri, B.T. Nguyen, I. Grappasonni, A systematic review of pharmacoeconomic evaluations of erlotinib in the first-line treatment of advanced non-small cell lung cancer, Eur J Health Econ 20(5) (2019) 763-777.

11. S.J. Seung, M. Hurry, S. Hassan, R.N. Walton, W.K. Evans, Cost-of-illness study for non-small-cell lung cancer using real-world data, Curr Oncol 26(2) (2019) 102-107.

12. C.M. Cramer-van der Welle, B.J.M. Peters, F. Schramel, O.H. Klungel, H.J.M. Groen, E.M.W. van de Garde, Santeon NSCLC Study Group, Systematic evaluation of the efficacy-effectiveness gap of systemic treatments in metastatic nonsmall cell lung cancer, Eur Respir J 52(6) (2018).

13. E.M.W. van de Garde, B.C. Plouvier, H. Fleuren, E.A.F. Haak, K.L.L. Movig, M.J. Deenen, M. van Hulst, Pharmacotherapy within a learning healthcare system: rationale for the Dutch Santeon Farmadatabase, Eur J Hosp Pharm 26(1) (2019) 46-50.

14. Z-index BV. https://www.z-index.nl/english.

15. CBS. https://statline.cbs.nl/Statweb/?LA=en. Accessed Jan 28, 2019.

16. Institute for Medical Technology Assessment, Dutch manual for costing studies, Erasmus Universiteit Rotterdam, 2015

17. T.A. Kanters, C.A.M. Bouwmans, N. van der Linden, S.S. Tan, L. Hakkaart-van Roijen, Update of the Dutch manual for costing studies in health care, PLoS One 12(11) (2017) e0187477.

18. S.S. Tan, C.W. Van Gils, M.G. Franken, L. Hakkaart-van Roijen, C.A. Uyl-de Groot, The unit costs of inpatient hospital days, outpatient visits, and daycare treatments in the fields of oncology and hematology, Value Health 13(6) (2010) 712-9.

19. M.E. Charlson, P. Pompei, K.L. Ales, C.R. MacKenzie, A new method of classifying prognostic comorbidity in longitudinal studies: development and validation, J Chronic Dis 40(5) (1987) 373-83.

20. V. Sundararajan, T. Henderson, C. Perry, A. Muggivan, H. Quan, W.A. Ghali, New ICD-10 version of the Charlson comorbidity index predicted in-hospital mortality, J Clin Epidemiol 57(12) (2004) 1288-94.

21. T. Ciuleanu, T. Brodowicz, C. Zielinski, J.H. Kim, M. Krzakowski, E. Laack, Y.L. Wu, I. Bover, S. Begbie, V. Tzekova, B. Cucevic, J.R. Pereira, S.H. Yang, J. Madhavan, K.P. Sugarman, P. Peterson, W.J. John, K. Krejcy, C.P. Belani, Maintenance pemetrexed plus best supportive care versus placebo plus best supportive care for non-small-cell lung cancer: a randomised, double-blind, phase 3 study, Lancet 374(9699) (2009) 1432-40.

22. N. van der Linden, M.L. Bongers, V.M. Coupe, E.F. Smit, H.J. Groen, A. Welling, F.M. Schramel, C.A. Uyl-de Groot, Costs of non-small cell lung cancer in the Netherlands, Lung Cancer 91 (2016) 79-88. 
23. B.J.M. Peters, C.M. Cramer-Vd Welle, A.A.J. Smit, F. Schramel, E.M.W. van de Garde, Santeon NSCLC Study Group, Trends in prescribing systemic treatment and overall survival for non-small cell lung cancer stage IIIB/IV in the Netherlands: 2008-2012, Cancer Epidemiol 51 (2017) 1-6.

24. W.R. Keusters, V.A. de Weger, A. Hovels, J.H.M. Schellens, G.W.J. Frederix, Changing costs of metastatic non small cell lung cancer in the Netherlands, Lung Cancer 114 (2017) 56-61.

25. L. Gandhi, D. Rodriguez-Abreu, S. Gadgeel, E. Esteban, E. Felip, F. De Angelis, M. Domine, P. Clingan, M.J. Hochmair, S.F. Powell, S.Y. Cheng, H.G. Bischoff, N. Peled, F. Grossi, R.R. Jennens, M. Reck, R. Hui, E.B. Garon, M. Boyer, B. Rubio-Viqueira, S. Novello, T. Kurata, J.E. Gray, J. Vida, Z. Wei, J. Yang, H. Raftopoulos, M.C. Pietanza, M.C. Garassino, K.-. Investigators, Pembrolizumab plus Chemotherapy in Metastatic Non-Small-Cell Lung Cancer, N Engl J Med 378(22) (2018) 2078-2092.

26. L. Paz-Ares, A. Luft, D. Vicente, A. Tafreshi, M. Gumus, J. Mazieres, B. Hermes, F. Cay Senler, T. Csoszi, A. Fulop, J. Rodriguez-Cid, J. Wilson, S. Sugawara, T. Kato, K.H. Lee, Y. Cheng, S. Novello, B. Halmos, X. Li, G.M. Lubiniecki, B. Piperdi, D.M. Kowalski, K.-. Investigators, Pembrolizumab plus Chemotherapy for Squamous Non-Small-Cell Lung Cancer, N Engl J Med 379(21) (2018) 2040-2051.

27. M.A. Socinski, R.M. Jotte, F. Cappuzzo, F. Orlandi, D. Stroyakovskiy, N. Nogami, D. Rodriguez-Abreu, D. Moro-Sibilot, C.A. Thomas, F. Barlesi, G. Finley, C. Kelsch, A. Lee, S. Coleman, Y. Deng, Y. Shen, M. Kowanetz, A. Lopez-Chavez, A. Sandler, M. Reck, I.M.S. Group, Atezolizumab for First-Line Treatment of Metastatic Nonsquamous NSCLC, N Engl J Med 378(24) (2018) 2288-2301.

28. W. Luo, Z. Wang, P. Tian, W. Li, Safety and tolerability of PD-1/PD-L1 inhibitors in the treatment of non-small cell lung cancer: a meta-analysis of randomized controlled trials, J Cancer Res Clin Oncol 144(10) (2018) 1851-1859.

29. D.E. Magee, A.E. Hird, Z. Klaassen, S.S. Sridhar, R.K. Nam, C.J.D. Wallis, G.S. Kulkarni, Adverse event profile for immunotherapy agents compared with chemotherapy in solid organ tumors: a systematic review and meta-analysis of randomized clinical trials, Ann Oncol 31(1) (2020) 50-60.

30. M.E. Porter, What is value in health care?, N Engl J Med 363(26) (2010) 2477-81.

31. S.S. Godman B., Pricing of oral generic cancer medicines in 25 European countries; findings and implications, Generics and Biosimilars Initiative Journal (GaBl Journal) 8(2) (2019) 49-70. 


\section{APPENDIX CHAPTER 4}

Table 2. Unit costs per mg drug per year

\begin{tabular}{lccccccccc}
\hline & $\mathbf{2 0 0 8}$ & $\mathbf{2 0 0 9}$ & $\mathbf{2 0 1 0}$ & $\mathbf{2 0 1 1}$ & $\mathbf{2 0 1 2}$ & $\mathbf{2 0 1 3}$ & $\mathbf{2 0 1 4}$ & $\mathbf{2 0 1 5}$ & $\mathbf{2 0 1 6}$ \\
\hline Pemetrexed & 4.25 & $\mathbf{4 . 2 0}$ & 3.53 & 3.15 & 3.05 & 3.06 & 3.02 & 3.01 & 3.06 \\
Bevacizumab & 4.14 & $\mathbf{4 . 0 4}$ & 3.79 & 3.73 & 3.62 & 3.60 & 3.50 & 3.45 & 3.57 \\
Carboplatin & 0.90 & 0.89 & 0.88 & 0.86 & 0.84 & 0.27 & 0.26 & 0.26 & 0.26 \\
Cisplatin & 1.07 & 1.06 & 0.82 & 0.56 & 0.56 & 0.52 & 0.51 & 0.51 & 0.51 \\
Docetaxel & 11.75 & 11.19 & 10.55 & 4.50 & 4.39 & 4.28 & 5.24 & 5.21 & 5.19 \\
Etoposide & 0.21 & 0.20 & 0.20 & 0.20 & 0.19 & 0.17 & 0.16 & 0.16 & 0.15 \\
Gemcitabin & 0.26 & 0.24 & 0.11 & 0.11 & 0.10 & 0.05 & 0.05 & 0.05 & 0.05 \\
Paclitaxel & 0.98 & 0.97 & 0.96 & 0.93 & 2.82 & 2.75 & 2.72 & 2.71 & 2.70 \\
Vinorelbin & 2.30 & 2.27 & 2.24 & 2.19 & 2.14 & 2.08 & 2.56 & 2.53 & 2.53 \\
Erlotinib & 0.60 & 0.59 & 0.57 & 0.57 & 0.54 & 0.53 & 0.53 & 0.53 & 0.55 \\
Gefitinib & $\mathrm{n} / \mathrm{a}$ & $\mathrm{n} / \mathrm{a}$ & 0.42 & 0.39 & 0.38 & 0.38 & 0.37 & 0.37 & 0.38 \\
Afatiniba & $\mathrm{n} / \mathrm{a}$ & $\mathrm{n} / \mathrm{a}$ & $\mathrm{n} / \mathrm{a}$ & $\mathrm{n} / \mathrm{a}$ & $\mathrm{n} / \mathrm{a}$ & 95.64 & 93.54 & 91.84 & 90.04 \\
Crizotinib & $\mathrm{n} / \mathrm{a}$ & $\mathrm{n} / \mathrm{a}$ & $\mathrm{n} / \mathrm{a}$ & $\mathrm{n} / \mathrm{a}$ & $\mathrm{n} / \mathrm{a}$ & 0.40 & 0.40 & 0.40 & 0.39 \\
Ceritinib & $\mathrm{n} / \mathrm{a}$ & $\mathrm{n} / \mathrm{a}$ & $\mathrm{n} / \mathrm{a}$ & $\mathrm{n} / \mathrm{a}$ & $\mathrm{n} / \mathrm{a}$ & $\mathrm{n} / \mathrm{a}$ & $\mathrm{n} / \mathrm{a}$ & $\mathrm{n} / \mathrm{a}$ & 0.30 \\
Nintedanib & $\mathrm{n} / \mathrm{a}$ & $\mathrm{n} / \mathrm{a}$ & $\mathrm{n} / \mathrm{a}$ & $\mathrm{n} / \mathrm{a}$ & $\mathrm{n} / \mathrm{a}$ & $\mathrm{n} / \mathrm{a}$ & $\mathrm{n} / \mathrm{a}$ & 0.31 & 0.31 \\
Osimertiniba & $\mathrm{n} / \mathrm{a}$ & $\mathrm{n} / \mathrm{a}$ & $\mathrm{n} / \mathrm{a}$ & $\mathrm{n} / \mathrm{a}$ & $\mathrm{n} / \mathrm{a}$ & $\mathrm{n} / \mathrm{a}$ & $\mathrm{n} / \mathrm{a}$ & $\mathrm{n} / \mathrm{a}$ & 222.27 \\
\hline
\end{tabular}

Data are presented as $€$ per mg drug including VAT and inflation rates

aFixed price per day, dose independent 



\section{Chapter 5}

Real-world outcomes versus clinical trial results of immunotherapy in stage IV non-small cell lung cancer (NSCLC) in the Netherlands

Christine M. Cramer-van der Welle, Marjon V. Verschueren, Merel Tonn, Bas J.M. Peters, Franz M.N.H. Schramel, Olaf H. Klungel, Harry J.M. Groen, Ewoudt M.W. van de Garde, and the Santeon NSCLC Study Group

Accepted for publication in Scientific Reports 


\section{ABSTRACT}

Purpose: This study aims to assess how clinical outcomes of immunotherapy in real-world (effectiveness) correspond to outcomes in clinical trials (efficacy) and to look into factors that might explain an efficacy-effectiveness (EE) gap.

Patients and Methods: All patients diagnosed with stage IV NSCLC in 2015-2018 in six Dutch large teaching hospitals (Santeon network) were identified and followed-up from date of diagnosis until death or end of data collection. Progression-free survival (PFS) and overall survival (OS) from first-line (1L) pembrolizumab and second-line (2L) nivolumab were compared with RCT data by calculating hazard ratios (HRs).

Results: From 1950 diagnosed patients, 1005 (52\%) started with any 1L treatment, of which 83 received pembrolizumab. Nivolumab was started as 2 L treatment in 141 patients. For both settings, PFS times were comparable between real-world and trials (HR 1.08 (95\%Cl 0.75-1.55), and HR 0.91 (95\%Cl 0.74-1.14), respectively). OS was significantly shorter in real-world for $1 \mathrm{~L}$ pembrolizumab (HR 1.55; 95\% Cl 1.07-2.25). Receiving subsequent lines of treatment was less frequent in real-world compared to trials.

Conclusion: There is no EE gap for PFS from immunotherapy in patients with stage IV NSCLC. However, there is a gap in OS for $1 \mathrm{~L}$ pembrolizumab. Fewer patients proceeding to a subsequent line of treatment in real-world could partly explain this. 


\section{INTRODUCTION}

For patients with stage IV NSCLC, immune checkpoint inhibitors (ICls) have demonstrated promising results in disease progression and survival in randomized clinical trials $(R C T s)^{1-6}$. Currently, there are three FDA and EMA approved PD-1/PD-L1 inhibitors available for treatment of stage IV NSCLC. These include nivolumab (anti-PD-1), pembrolizumab (anti-PD-1), and atezolizumab (anti PD-L1)1. However, results from RCTs are not easily generalizable to clinical practice due to strict eligibility criteria ${ }^{7-9}$. While the population is ageing, which is associated with an increased risk of lung cancer, most patients that are included in RCTs are under 65 years old 10,11. Additionally, NSCLC patients with comorbidities, especially auto-immune diseases, or poor performance status are often not eligible for participating in RCTs ${ }^{11,12}$. At present, RCTs are still the gold standard to prove efficacy for new oncology therapies, 7 . Consequently, there could be a gap between the efficacy demonstrated in RCTs and the effectiveness of systemic therapies in clinical practice.

Recently, we have demonstrated that survival of patients with metastatic NSCLC treated with chemotherapy or targeted therapy is nearly one-quarter shorter in real-world practice than for patients included in trials ${ }^{13}$. For immunotherapy in metastatic NSCLC, some efforts have been made as well to provide insight into this possible gap. So far, most studies reported comparable outcomes in real-world compared to those observed in clinical trials. For example, a recent study from The Netherlands by Smit et al. ${ }^{14}$ assessed real-world effectiveness of ICls in a Dutch population of patients with advanced NSCLC and showed similar median survival times as those of phase III studies, except for patients with ECOG performance status (PS) of $\geq 2$. The latter study, however, did not discriminate between type of drug nor compared survival curves to detect potential differences between survival dynamics in real-world and trials. Many studies have shown that the shape of the survival curve for immunotherapy is different than for chemotherapy, with some extra early deaths with immunotherapy and a plateau of long survivors later on. Furthermore, patient characteristics have not been compared with trial cohorts in search for explanatory factors for any efficacy-effectiveness gap.

The aim of this study is to assess how clinical outcomes of specific immunotherapies for patients with stage IV NSCLC in real-world (effectiveness) correspond to the results from clinical trials (efficacy) and to look into factors that might explain an efficacy-effectiveness gap. 


\section{PATIENTS AND METHODS}

\section{Data sources}

Clinical data from six hospitals of the Santeon hospital network were used for this study. This hospital network consists of seven large (non-university) teaching hospitals that are geographically spread over The Netherlands and serve $>11 \%$ of the Dutch population ${ }^{15}$. Patients diagnosed with stage IV NSCLC were identified from the Netherlands Cancer Registry $(\mathrm{NCR})^{16}$. Data on diagnosis, vital status, and patient characteristics were obtained from the NCR.

Data on pharmacotherapy from the Santeon Farmadatabase was merged with the NCR dataset, to construct an overview of all applied regimes in the study population. The Santeon Farmadatabase contains, among others, information on individual prescriptions with drug name, dosage, date of administration and administration route ${ }^{17}$. Patients' medical records were used to complement and validate the dataset.

Data was collected and managed with REDCap ${ }^{18}$, hosted at St. Antonius Hospital, Utrecht/ Nieuwegein, the Netherlands.

\section{Study population}

Patients with stage IV NSCLC diagnosed between January 1st, 2015 and December 31st, 2018 in one of six Santeon Hospitals were selected. Staging was based on the 7th edition TNM classification for the years 2015-2016 and 8th edition for incidence years 2017-2018. Characteristics that were recorded for included patients were age (at year of diagnosis), gender, ECOG PS, histology, brain metastases, pre-existing autoimmune disease, and PD-L1 expression of the tumour. Also, the type of treatment (best supportive care (BSC), chemotherapy, targeted therapy or immunotherapy), line of treatment, hospital where the patient was treated, and (in case of immunotherapy) information on immune related adverse events (irAE) and palliative radiotherapy were collected.

\section{Identification of systemic treatments per patient}

First-line treatment was defined as the initial systemic treatment that was started after diagnosis. Second-line or further line treatment was defined as systemic treatment applied after completion or discontinuation because of disease progression of first or second-line treatment, respectively. After the identification of all different regimens we ordered patients in four different categories: chemotherapy, treatment with tyrosine kinase inhibitors (TKIs), immunotherapy, or best supportive care. For this study we focussed on immunotherapy and identified the most frequently used types of drugs (pembrolizumab and nivolumab) in first and second-line in our database. Second-line nivolumab for NSCLC in the Netherlands was introduced in March 2016. In 2017, first-line pembrolizumab for NSCLC with PD-L1 TPS $\geq 50 \%$ was introduced. 


\section{Reference outcome}

After identification of the most commonly used types of immunotherapy, corresponding reference outcomes were established from clinical trials. These clinical trials were identified by a literature search on PubMed for clinical trials used for approval of immunotherapy drugs. If multiple registration studies were published with different patient populations, the study with the most comparable patient population (based on the distribution of stage, PD-L1 expression, and histology) to our cohort was chosen. We searched for updated publications of the selected studies (if applicable) to use up-to-date data and to utilize as much as possible of their follow-up time for the comparison with real-world data.

\section{Statistical analysis}

All statistical analyses were conducted using R software package version 3.6.1.

To present an overview of baseline characteristics for all treated patients, frequencies (proportions) were calculated for categorical variables, and means (standard deviations) were provided for normally distributed continuous data.

For patients who received immunotherapy in first or second-line, overall survival (OS) was calculated as time between start date of treatment until date of death from any cause. Patients still alive at January 1, 2020 were censored as this was the end of follow-up date. Progression free survival (PFS) was calculated as time between start date of treatment until the occurrence of progression according to Response Evaluation Criteria in Solid Tumours (RECIST criteria) when noted. Date of death was noted in absence of acknowledged progression from the individual patient files. Survival curves were obtained for the treatment groups using the Kaplan-Meier method.

The potential existence of an efficacy-effectiveness gap was assessed in two manners. First, a so-called efficacy-effectiveness factor (EE factor) was calculated by dividing the patient's individual median survival by the corresponding reference OS and PFS from clinical trials ${ }^{13}$. This factor was used to estimate the presence of an efficacy-effectiveness gap and shows how the patient's individual survival is related to survival presented in the reference RCT. As an example, an EE factor of 0.70 shows that median survival is $30 \%$ shorter in clinical practice than in RCTs. The Wilcoxon signed rank test was used to analyse the distribution of the EE factors. The null hypothesis (median OS in real-world is similar to median OS reported in clinical trials) is rejected if the distribution is significantly different from test value 1.0 .

Second, we assessed hazard ratios (HRs) between real-world immunotherapy treatment regimens and groups in corresponding RCTs to compare OS and PFS. This was achieved by first digitizing the Kaplan-Meier (KM) curves for the immunotherapy arm from the included clinical trials with the R-package 'digitize'. The extracted data points for survival probability were used for reconstructing the KM curve with the algorithm as described by Guyot et al..$^{19}$ The coordinates of the KM curve from the published graph were read in with the R-coding script by Guyot et al., together with the information on numbers at risk and 
total number of events, to reconstruct the KM data. With this reconstructed individual patient data (IPD), KM curves and Cox HRs were estimated using the R routines 'survfit' and 'coxph'.

Univariate and multivariate analyses for potential prognostic factors for PFS and OS in real-world were performed using Cox proportional hazards regression models. The factors with $p<0.05$ on univariate analysis were included in the multivariate analysis. In this analysis, missing values were imputed by single stochastic regression imputation (single run with all available characteristics in the model). Pearson's chi-square tests were performed to test if the proportion of the prognostic factor in real-world differed from reference $\mathrm{RCT}$ data, if applicable.

\section{Ethical approval}

The study was approved by each individual Institutional Review Board of the six Santeon Hospitals (Catharina Hospital Eindhoven, Canisius Wilhelmina Hospital Nijmegen, Martini Hospital Groningen, Medisch Spectrum Twente Enschede, OLVG Amsterdam, and St. Antonius Hospital Utrecht/Nieuwegein). The need for informed consent was waved because of anonymous data handling and the retrospective nature of the study. The study was performed in accordance with the ethical standards of the institutional and national research committee and with the 1964 Helsinki declaration and its later amendments or comparable ethical standards.

\section{RESULTS}

\section{Baseline characteristics}

We identified 1950 patients diagnosed with stage IV NSCLC in the period 2015 to 2018. Figure 1 provides an overview of the different treatment patterns of all patients towards best supportive care (BSC). Of these patients, 922 (47\%) did not receive active anti-tumour treatment in $1 \mathrm{~L}$ because of their ECOG PS, comorbidities, or at request of the patient. Twenty-three patients (1\%) were referred to other hospitals after diagnosis of whom we did not have information on treatment. Of all diagnosed patients, 1005 (52\%) received $1 \mathrm{~L}$ treatment of which 92 (9\%) received immunotherapy in first-line. Of all treated patients, only 365 (36\%) received subsequent treatment in one of the six hospitals of which 200 (55\%) received immunotherapy. The most frequently received $1 \mathrm{~L}$ immunotherapy was pembrolizumab ( $n=83,90 \%$ of all patients treated with immunotherapy in $1 \mathrm{~L}$ ) and in second-line nivolumab for patients with non-squamous tumour histology ( $n=141,71 \%$ ). Based on these subgroups, two registration studies (Checkmate 057 and KEYNOTE-024 2,5) were identified for the comparison with real-world data.

Table 1 shows patient characteristics of patients with $1 \mathrm{~L}$ pembrolizumab or $2 \mathrm{~L}$ nivolumab (both monotherapy). The median age of patients with $1 \mathrm{~L}$ pembrolizumab was 66 years 
Table 1. Patient characteristics of stage IV NSCLC patients with $1 \mathrm{~L}$ pembrolizumab or 2 L nivolumab.

\begin{tabular}{|c|c|c|c|c|}
\hline & \multicolumn{2}{|c|}{ Pembrolizumab $1^{\text {st }}$ line } & \multicolumn{2}{|c|}{ Nivolumab $2^{\text {nd }}$ line } \\
\hline & Real-world & $\mathrm{RCT}^{5}$ & Real-world & $\mathrm{RC} \mathrm{T}^{2}$ \\
\hline Subjects & 83 & 154 & 141 & 292 \\
\hline Male & $45(54 \%)$ & $92(60 \%)$ & $74(53 \%)$ & $151(52 \%)$ \\
\hline Age (median (range)) & $66.0(35-84)$ & $64.5(33-90)$ & $64.0(44-80)$ & $61.0(37-84)$ \\
\hline \multicolumn{5}{|l|}{ ECOG PS } \\
\hline 0 & $30(36 \%)$ & $54(35 \%)$ & $44(31 \%)$ & 84 (29\%) \\
\hline 1 & $50(60 \%)$ & 99 (64\%) & $90(64 \%)$ & 208 (71\%) \\
\hline 2 & $3(4 \%)$ & $1(1 \%)$ & $7(5 \%)$ & - \\
\hline \multicolumn{5}{|l|}{ Histology } \\
\hline Squamous & $11(13)$ & $29(19 \%)$ & - & - \\
\hline Non-squamous & $72(87)$ & 125 (81\%) & $141(100 \%)$ & $292(100.0)$ \\
\hline \multicolumn{5}{|l|}{ PD-L1 expression } \\
\hline$<1 \%$ & - & - & $33(23 \%)$ & 108 (37\%) \\
\hline $1-49 \%$ & - & - & $29(21 \%)$ & $123^{*}(42 \%)$ \\
\hline$\geq 50 \%$ & $83(100 \%)$ & $154(100 \%)$ & $10(7 \%)$ & \\
\hline Unknown*** & - & - & 69 (49\%) & $61(21 \%)$ \\
\hline CNS metastases & $16(19 \%)$ & $18(12 \%)$ & $25(18 \%)$ & $34(12 \%)$ \\
\hline $\begin{array}{l}\text { Subsequent systemic } \\
\text { therapy }\end{array}$ & $14(17 \%)$ & $56(36 \%)^{* *}$ & $40(28 \%)$ & $123(42 \%)$ \\
\hline
\end{tabular}

* PD-L1 expression of $\geq 1 \%$. ${ }^{* *}$ Including surgery and radiation therapy. ${ }^{* *}$ Not performed in real-world; not quantifiable from RCT2.

ECOG PS: Eastern Cooperative Oncology Group Performance Status; CNS: central nervous system; RCT: randomized clinical trial.

and almost all patients (96\%) had an ECOG PS of 0 or 1. The median age of patients with $2 \mathrm{~L}$ nivolumab was 64 years and $95 \%$ had an ECOG PS of 0 or 1.

\section{Survival}

Median PFS and OS for $1 \mathrm{~L}$ pembrolizumab treatment were 8.9 and 15.8 months, respectively. For patients treated with $2 \mathrm{~L}$ nivolumab, median PFS was 3.8 and median OS was 8.2 months (Table 2). One-year survival was $57 \%$ for $1 \mathrm{~L}$ pembrolizumab and $42 \%$ for $2 \mathrm{~L}$ nivolumab. In the corresponding clinical trials on pembrolizumab and nivolumab, one-year survival was $70 \%$ and $51 \%$, respectively 2,20 .

\section{EE factor analysis}

Median OS was shorter for all patients who received $1 \mathrm{~L}$ pembrolizumab in real-world practice compared to the clinical trial. Table 2 shows an EE factor of 0.45 ( $p<0.001$ from 1), 
which means that median survival is 55\% shorter for patients treated in clinical practice relative to median survival from the registration clinical trial. There was no significant difference in median PFS for $1 \mathrm{~L}$ pembrolizumab, with an EE factor of $0.85(p=0.86)$.

For $2 \mathrm{~L}$ nivolumab, the EE factor for median OS is 0.65 ( $p=0.065)$. For PFS, we found an EE factor of $1.61(p<0.001)$ (Table 2). This indicates that median PFS is $61 \%$ higher for nivolumab in clinical practice, compared to median PFS from the RCT.

Table 2. Median survival in real-world clinical practice and randomized clinical trials

\begin{tabular}{llll}
\hline & $\begin{array}{l}\text { Real-world } \\
\text { months }(95 \% \mathrm{Cl})\end{array}$ & $\begin{array}{l}\text { Clinical trial } \\
\text { months }(95 \% \mathrm{Cl})\end{array}$ & $\begin{array}{l}\text { EE factor } \\
\text { median }\end{array}$ \\
\hline $\begin{array}{l}\text { 1L Pembrolizumab } \\
\text { mPFS }\end{array}$ & $\begin{array}{l}\text { (9.9 }(3.7-14.1) \\
\text { mOS }\end{array}$ & $10.3(6.7-\mathrm{NR})^{5}$ & 0.85 \\
2L Nivolumab & $15.8(9.4-22.1)$ & $30.0(18.3-\mathrm{NR})^{33}$ & $0.45^{\mathrm{a}}$ \\
mPFS & $3.8(3.0-4.7)$ & & \\
mOS & $8.2(5.9-10.6)$ & $12.2(9.7-15.1)^{34}$ & 0.65 \\
\hline
\end{tabular}

mPFS: median progression-free survival; mOS: median overall survival; EE factor: efficacy-effectiveness factor. a significantly different ( $p$-values $<0.05$ ) from test value 1.00 (one-sample Wilcoxon signed-rank test)

\section{Proportional hazard analysis}

The estimated HRs for $1 \mathrm{~L}$ pembrolizumab in real-world compared to data from the RCT were $1.08(95 \% \mathrm{Cl} 0.75-1.55)$ and $1.55(95 \% \mathrm{Cl} 1.07-2.25)$ for PFS and OS, respectively (Figure 2). For $2 \mathrm{~L}$ nivolumab the estimated HRs were 0.91 (95\% Cl 0.74-1.14) for PFS and $1.17(95 \% \mathrm{Cl}$ 0.93-1.48) for OS (Figure 3). This indicates that PFS is comparable between real-world and RCTs, and in real-world OS is significantly shorter for $1 \mathrm{~L}$ immunotherapy.

From the univariate cox proportional hazards regression model, no significant associations were found with PFS or OS for all potential prognostic factors in real-world patients with $1 \mathrm{~L}$ pembrolizumab, or for PFS in real-world patients with $2 \mathrm{~L}$ nivolumab. Higher ECOG PS (0-1 vs. $\geq 2)$ at start of $2 \mathrm{~L}$ nivolumab was associated with shorter OS (HR 2.23; $95 \% \mathrm{Cl}$ 1.03-4.83; $p=0.043)$. Further analysis showed that the proportion of patients with ECOG PS $\geq 2$ in real-world is higher compared to patients in clinical trials ( $5 \%$ versus $0 \%, p<0.001$ ).

When looking at explanatory factors after starting $1 \mathrm{~L}$ pembrolizumab in real-world, we found that PFS was influenced by the occurrence of irAEs. Having an irAE reduces the hazard by a factor of 0.48 ( $95 \% \mathrm{Cl} 0.26-0.89 ; \mathrm{p}=0.019)$. For $2 \mathrm{~L}$ nivolumab, the occurrence of irAEs was also an explanatory factor for longer PFS and OS (HR 0.45; 95\%Cl 0.30-68; $p<0.001$ and $\mathrm{HR} 0.44 ; 95 \% \mathrm{Cl} 0.27-0.70 ; \mathrm{p}<0.001$, respectively). 


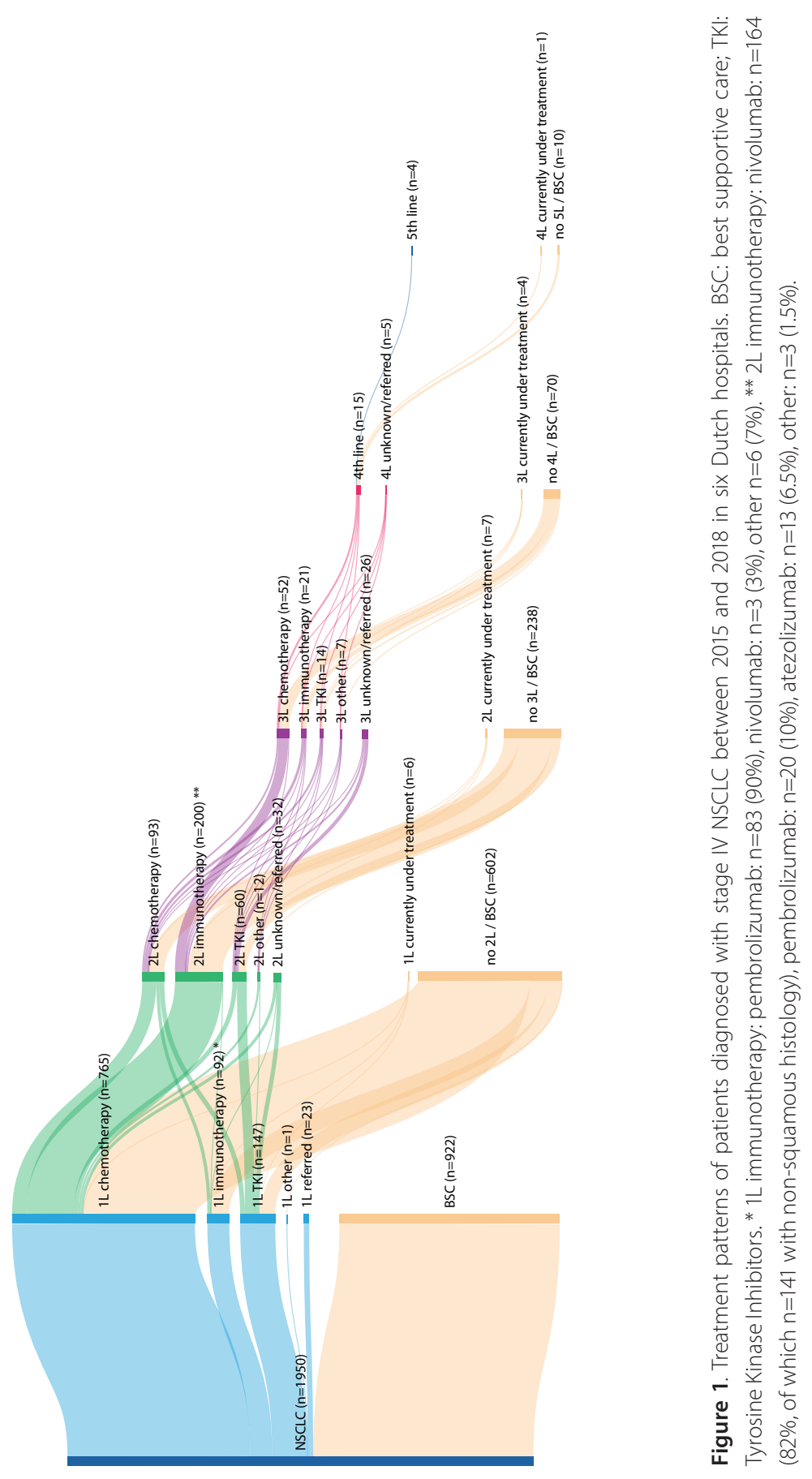



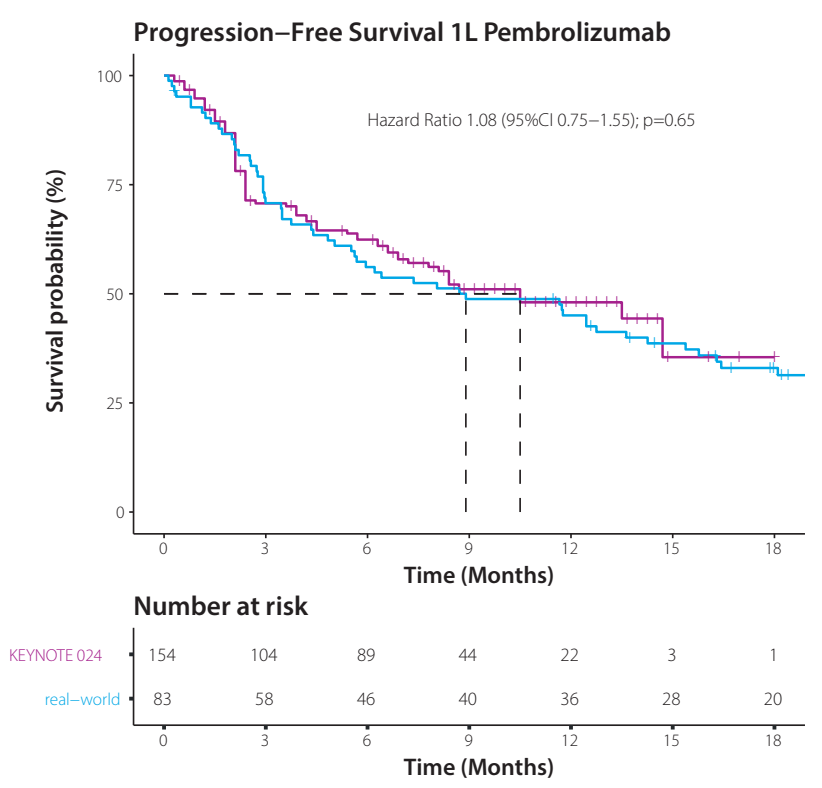

+ KEYNOTE 024 + real-world

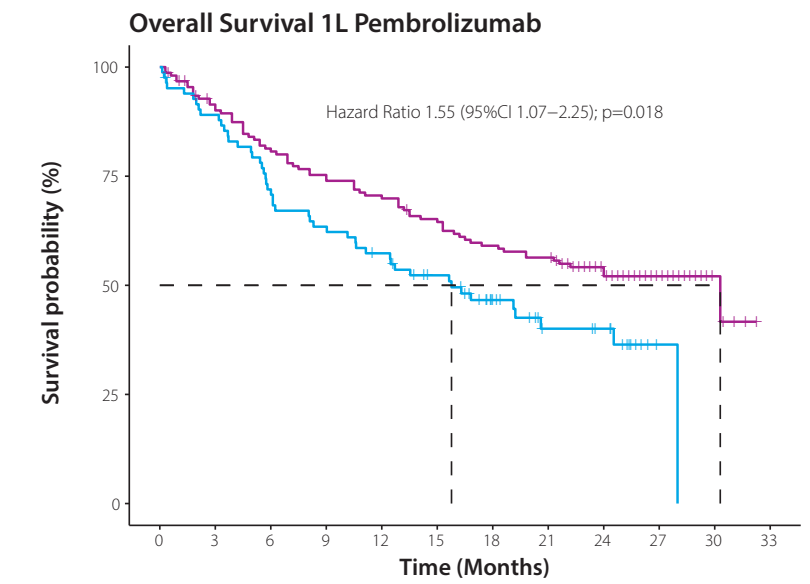

Number at risk

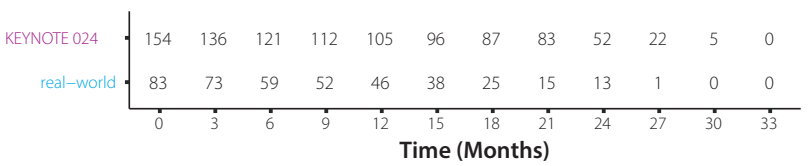

+ KEYNOTE 024 + real-world

Figure 2. Kaplan-Meier curves of PFS and OS in patients receiving $1 \mathrm{~L}$ pembrolizumab in real-world and clinical trial 

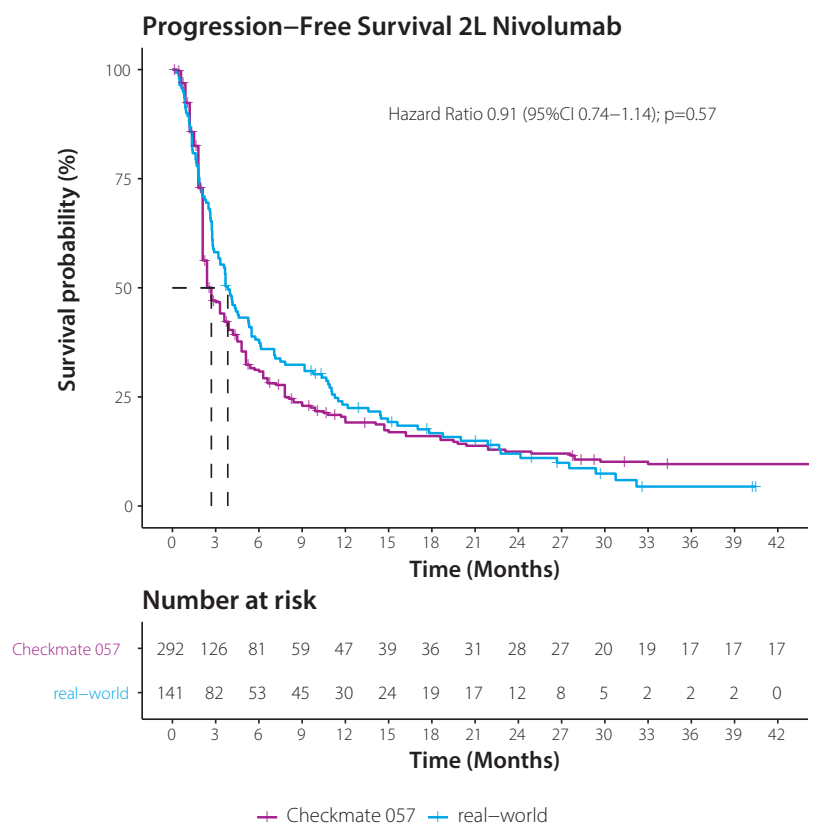

Overall Survival 2L Nivolumab

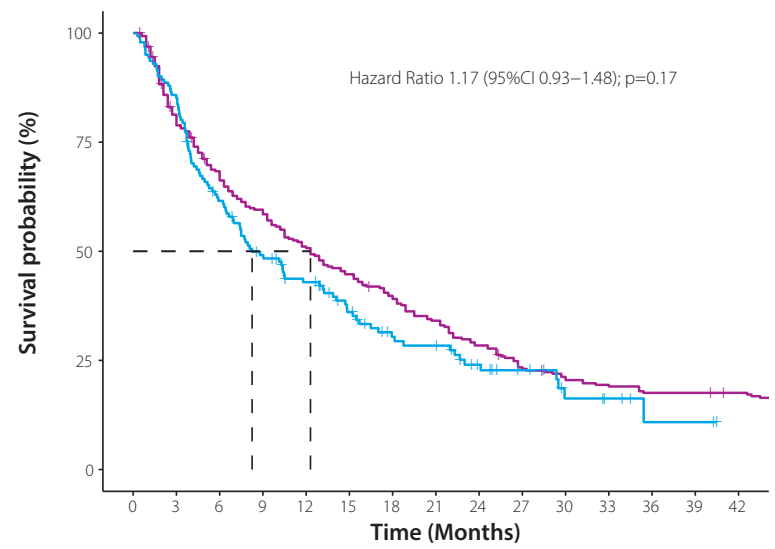

Number at risk

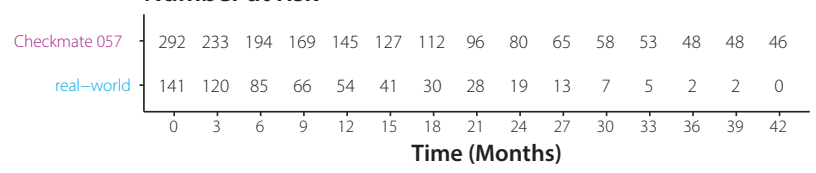

+ Checkmate 057 + real-world

Figure 3. Kaplan-Meier curves of PFS and OS in patients receiving $2 \mathrm{~L}$ nivolumab in real-world and clinical trial 
In real-world, the proportion of patients with irAEs was significantly lower compared to clinical trials for both $1 \mathrm{~L}$ pembrolizumab and $2 \mathrm{~L}$ nivolumab (31\% versus $77 \%$ with $p<0.001$, and $28 \%$ versus $69 \%$ with $p<0.001$, respectively). Furthermore, fewer patients received a subsequent line of treatment in real-world compared to patients in clinical trials after both $1 \mathrm{~L}$ pembrolizumab and $2 \mathrm{~L}$ nivolumab (17\% versus $36 \%$ with $\mathrm{p}=0.002$, and $28 \%$ versus $42 \%$ with $\mathrm{p}=0.014$, respectively).

\section{DISCUSSION}

This study showed that PFS of patients with stage IV NSCLC treated with immunotherapy is comparable between real-world and clinical trials. However, OS is significantly shorter for patients with 1L pembrolizumab in real-world (median 15.8 vs. 30 months; HR 1.55 $(95 \% \mathrm{Cl} 1.07-2.25))$. This finding is in line with our previous research ${ }^{13}$ which showed that survival after first-line chemotherapy and targeted therapy is around one quarter shorter in real-world compared to clinical trial data. The present study extends this finding to first-line pembrolizumab monotherapy in patients with $\geq 50 \%$ PD-L1 expression.

To our knowledge, this is the first study that calculated hazard ratios for PFS and OS of real-world versus clinical trials. This approach provided insight that stage IV NSCLC patients who are treated with immunotherapy in regular clinical practice have a comparable period of PFS time but that this does not extend to a similar OS benefit as demonstrated in the registration trial. This implies that something differs between regular practice and clinical trial participants after progression on immunotherapy. One explanation from our data could be the receiving of a next line of treatment being two times more frequent in clinical trial participants. Patients who received immunotherapy through participating in a clinical trial, for example, might have an above average intrinsic willingness to search for further treatment options after treatment failure. Other possible explanations could be that clinics active in trial enrolment communicate more about remaining experimental treatments, or that patients characteristics, other than performance status, limit the tolerability for subsequent systemic treatment (in this case chemotherapy) after progression on immunotherapy to a larger extent in regular care patients.

The results on median OS and PFS as found in our study are largely in line with previous observational studies on $1 \mathrm{~L}$ immunotherapy treatment with pembrolizumab in real-world. Velcheti et al.20 evaluated real-world survival in patients with metastatic NSCLC with an PD-L1 expression of $\geq 50 \%$ and ECOG PS $\leq 1$, and found a median PFS of 6.8 months and median OS of 19.1 months. Their PFS and OS are slightly shorter than found in the KEYNOTE-024 clinical trial, but real-world OS is longer than was found in our study, which could be explained by only including patients with ECOG PS 0 or 1, or by the higher 
percentage of patients with second-line systemic therapy as compared to our cohort ( $28 \%$ vs. $17 \%$, respectively). A French study which included patients with advanced NSCLC and PD-L1 expression of $\geq 50 \%$, reported a median PFS of 10.1 months and a median OS of 15.2 months for patients treated with pembrolizumab in first-line, including patients with brain metastases and ECOG PS $2^{21}$. As in the results of the current study, their PFS is comparable with the median PFS from the KEYNOTE-024 trial of 10.3 months, whereas OS is shorter (comparable to our real-world findings). Tambo et al.22 found a median of 6.1 for PFS which is shorter than the median PFS from both the registration study and the current study. Shorter PFS may be explained by inclusion of patients with ECOG PS 2 (12\%) and 3-4 (11\%). Their OS did not reach the median and therefore could not be compared.

The outcomes found for $2 \mathrm{~L}$ nivolumab are also in line with previous studies with real-world data. Crinò et al. 23 found a median PFS for patients treated with nivolumab in second or further line of 4.2 months and a median OS of 7.9 months, which is comparable with the present findings (3.8 and 8.2 months, respectively). Another study in Dutch patients treated with second-line nivolumab reported a median PFS and OS of 2.6 and 10.0 months, respectively ${ }^{24}$. Grossi et al. 25 reported results from the expanded access program in Italy on 1588 patients, including patients with ECOG PS 2 and aged $\geq 75$ years, where median PFS was 3.0 months and median OS 11.3 months. The results of those three studies on real-world nivolumab in second-line suggest that results for PFS and OS in real-world and RCTs are indeed comparable. Interesting from our data is that the median PFS from $2 \mathrm{~L}$ nivolumab was significantly longer than in the clinical trial but without any difference in overall hazard (HR 0.91). An explanation could be a difference in how date of progression is determined between the two settings. But this finding also highlights that comparing median PFS times only could lead to biased conclusions about relative effectiveness.

Regarding prognostic factors, for patients treated with $2 \mathrm{~L}$ nivolumab, the negative association between a higher/worse ECOG PS and OS is in line with previous research. Crinò et al. ${ }^{23}$ also found ECOG PS to be a poor prognostic factor. Additionally, the results from Schouten et al. ${ }^{24}$ from the Dutch expanded access program and the study by Martin et al. ${ }^{26}$ show similar results. A Dutch study on real-world effectiveness of ICls in patients with stage IV NSCLC in first and second-line also shows that an ECOG PS $\geq 2$ is a poor prognostic factor ${ }^{14}$. Additionally, in a phase 3B/4 community-based study of nivolumab monotherapy in previously treated patients with advanced NSCLC including patients with poor performance status (Checkmate 153), a median overall survival of 4.0 months was found in patients with ECOG PS ${ }^{27}$. Next to the association of ECOG PS $\geq 2$ and worse outcome for patients with $2 \mathrm{~L}$ nivolumab in real-world, the proportion of patients with ECOG PS $\geq 2$ in real-world is significantly higher compared to the clinical trial. This confirms the general thinking that trials select more fit patients, where patients with higher ECOG PS in clinical practice aim for immunotherapy as well, resulting in an EE gap. 
Besides this, our data also confirms the relationship between irAEs and outcomes of immunotherapy. Similar to Lisberg et al. ${ }^{28}$ who retrospectively analysed the relationship in patients that were treated with pembrolizumab in KEYNOTE-001, we found improved survival for patients with irAEs. Another study by Haratani et al. ${ }^{29}$ also revealed that irAEs were positively associated with survival outcome in nivolumab treated patients. Ricciuti et al. ${ }^{30}$ confirmed the positive relationship between the occurrence of irAEs and survival for patients with $2 \mathrm{~L}$ nivolumab as well.

The main strengths of this study are its complete and precise data, nationwide multicentre approach, and that PFS and OS were compared both through comparison of medians as well as through a proportional hazards approach. The latter method better addresses that many patients were still alive at time of conducting the analyses and provides a unique opportunity to compare survival dynamics not captured by a single value for median survival.

Limitations of this study are that (patient) factors potentially responsible for an efficacy-effectiveness gap could only be analysed on the cohort level instead of patient level, because individual patient data from the respective clinical trials were not available. When available these could have been analysed in a multivariable cox regression model. Besides this, the treatment patterns shown in Figure 1 do not match with current practice anymore (introduction of $1 \mathrm{~L}$ immunochemotherapy combination limiting the use of $2 \mathrm{~L}$ immunotherapy). Nevertheless, this does not compromise the relative effectiveness assessments within specific treatments as conducted in our study.

Apart from the well-known complexity of how to translate progression free survival times to overall survival when survival data of the trial cohort is not mature yet 31,32 , the present study shows that this translation can be even more complex later on when it comes to generalizability to real-world settings. Considering that cost-effectiveness assessments are often based on overall survival data from trials, our observed shorter overall survival time after $1 \mathrm{~L}$ pembrolizumab in real-world compared to the clinical trial data could provide an argument to rework the initial cost-effectiveness assessment.

\section{CONCLUSION}

There is no efficacy-effectiveness gap for the outcome PFS for immunotherapy in patients with stage IV NSCLC. However, there is a gap in OS for first-line pembrolizumab. Fewer patients proceeding to a subsequent line of treatment in real-world could partly explain this. PFS and OS results from clinical trials can differ in generalizability to regular clinical practice. 


\section{REFERENCES}

1. Herzberg, B., Campo, M. J. \& Gainor, J. F. Immune Checkpoint Inhibitors in Non-Small Cell Lung Cancer. Oncologist 22, 81-88, doi:10.1634/theoncologist.2016-0189 (2017).

2. Borghaei, H. et al. Nivolumab versus Docetaxel in Advanced Nonsquamous Non-Small-Cell Lung Cancer. N Engl J Med 373, 1627-1639, doi:10.1056/NEJMoa1507643 (2015).

3. Brahmer, J.et al. Nivolumab versus Docetaxel in Advanced Squamous-Cell Non-Small-Cell Lung Cancer. NEngl J Med 373, 123-135, doi:10.1056/NEJMoa1504627 (2015).

4. Herbst, R. S. et al. Pembrolizumab versus docetaxel for previously treated, PD-L1-positive, advanced non-small-cell lung cancer (KEYNOTE-010): a randomised controlled trial. Lancet 387, 1540-1550, doi:10.1016/ S0140-6736(15)01281-7 (2016).

5. Reck, M. et al. Pembrolizumab versus Chemotherapy for PD-L1-Positive Non-Small-Cell Lung Cancer. N Engl J Med 375, 1823-1833, doi:10.1056/NEJMoa1606774 (2016).

6. Rittmeyer, A. et al. Atezolizumab versus docetaxel in patients with previously treated non-small-cell lung cancer (OAK): a phase 3, open-label, multicentre randomised controlled trial. Lancet 389, 255-265, doi:10.1016/ S0140-6736(16)32517-X (2017).

7. Corrigan-Curay, J., Sacks, L. \& Woodcock, J. Real-World Evidence and Real-World Data for Evaluating Drug Safety and Effectiveness. JAMA 320, 867-868, doi:10.1001/jama.2018.10136 (2018).

8. Miksad, R. A. \& Abernethy, A. P. Harnessing the Power of Real-World Evidence (RWE): A Checklist to Ensure Regulatory-Grade Data Quality. Clin Pharmacol Ther 103, 202-205, doi:10.1002/cpt.946 (2018).

9. Rothwell, P. M. External validity of randomised controlled trials: "to whom do the results of this trial apply?". Lancet 365, 82-93, doi:10.1016/S0140-6736(04)17670-8 (2005).

10. Califano, R. et al. Immune checkpoint blockade for non-small cell lung cancer: What is the role in the special populations? Eur J Cancer 125, 1-11, doi:10.1016/j.ejca.2019.11.010 (2020).

11. Hurria, A. \& Kris, M. G. Management of lung cancer in older adults. CA Cancer J Clin 53, 325-341, doi:10.3322/ canjclin.53.6.325 (2003).

12. Al-Baimani, K. et al. Are clinical trial eligibility criteria an accurate reflection of a real-world population of advanced non-small-cell lung cancer patients? Curr Oncol 25, e291-e297, doi:10.3747/co.25.3978 (2018).

13. Cramer-van der Welle, C. M. et al. Systematic evaluation of the efficacy-effectiveness gap of systemic treatments in metastatic nonsmall cell lung cancer. Eur Respir J 52, doi:10.1183/13993003.01100-2018 (2018).

14. Smit, H. J. M. et al. Effects of checkpoint inhibitors in advanced non-small cell lung cancer at population level from the National Immunotherapy Registry. Lung Cancer 140, 107-112, doi:10.1016/j.lungcan.2019.12.011 (2020).

15. Santeon Hospital Group. Feiten en cijfers 2019, <https://santeon.nl/over-santeon/feiten-en-cijfers/> (2019).

16. Netherlands Comprehensive Cancer Organization. Netherlands Cancer Registry 2019, <www.cijfersoverkanker. $\mathrm{nl}>$ (2019).

17. van de Garde, E. M. W. et al. Pharmacotherapy within a learning healthcare system: rationale for the Dutch Santeon Farmadatabase. Eur J Hosp Pharm 26, 46-50, doi:10.1136/ejhpharm-2017-001329 (2019).

18. Harris, P. A. et al. Research electronic data capture (REDCap)--a metadata-driven methodology and workflow process for providing translational research informatics support. J Biomed Inform 42, 377-381, doi:10.1016/j. jbi.2008.08.010 (2009).

19. Guyot, P., Ades, A. E., Ouwens, M. J. \& Welton, N. J. Enhanced secondary analysis of survival data: reconstructing the data from published Kaplan-Meier survival curves. BMCMed Res Methodo/ 12, 9, doi:10.1186/1471-2288-12-9 (2012).

20. Velcheti, V. et al. Outcomes of first-line pembrolizumab monotherapy for PD-L1-positive (TPS >/=50\%) metastatic NSCLC at US oncology practices. Immunotherapy 11, 1541-1554, doi:10.2217/imt-2019-0177 (2019).

21. Amrane, K. et al. First-line pembrolizumab for non-small cell lung cancer patients with PD-L1 $>/=50 \%$ in a multicenter real-life cohort: The PEMBREIZH study. Cancer Med 9, 2309-2316, doi:10.1002/cam4.2806 (2020).

22. Tambo, Y. et al. Real-World Efficacy of First-Line Pembrolizumab in Patients With Advanced or Recurrent Non-Small-Cell Lung Cancer and High PD-L1 Tumor Expression. Clin Lung Cancer, doi:10.1016/j.cllc.2020.02.017 (2020). 
23. Crino, L. et al. Italian Cohort of Nivolumab Expanded Access Program in Squamous Non-Small Cell Lung Cancer: Results from a Real-World Population. Oncologist 24, e1165-e1171, doi:10.1634/theoncologist.20180737 (2019).

24. Schouten, R. D., Muller, M., de Gooijer, C. J., Baas, P. \& van den Heuvel, M. Real life experience with nivolumab for the treatment of non-small cell lung carcinoma: Data from the expanded access program and routine clinical care in a tertiary cancer centre-The Netherlands Cancer Institute. Lung Cancer 126, 210-216, doi:10.1016/j. lungcan.2017.11.012 (2018).

25. Grossi, F. et al. Real-life results from the overall population and key subgroups within the Italian cohort of nivolumab expanded access program in non-squamous non-small cell lung cancer. Eur J Cancer 123, 72-80, doi:10.1016/j.ejca.2019.09.011 (2019).

26. Martin, C. et al. Efficacy and Safety of Nivolumab in Previously Treated Patients With Non-Small-cell Lung Cancer: Real World Experience in Argentina. Clin Lung Cancer, doi:10.1016/j.cllc.2020.02.014 (2020).

27. Spigel, D. R. et al. Safety, Efficacy, and Patient-Reported Health-Related Quality of Life and Symptom Burden with Nivolumab in Patients with Advanced Non-Small Cell Lung Cancer, Including Patients Aged 70 Years or Older or with Poor Performance Status (CheckMate 153). J Thorac Oncol 14, 1628-1639, doi:10.1016/j. jtho.2019.05.010 (2019).

28. Lisberg, A. et al. Treatment-Related Adverse Events Predict Improved Clinical Outcome in NSCLC Patients on KEYNOTE-001 at a Single Center. Cancer Immunol Res 6, 288-294, doi:10.1158/2326-6066.CIR-17-0063 (2018).

29. Haratani, K. et al. Association of Immune-Related Adverse Events With Nivolumab Efficacy in Non-Small-Cell Lung Cancer. JAMA Onco/ 4, 374-378, doi:10.1001/jamaoncol.2017.2925 (2018).

30. Ricciuti, B. et al. Impact of immune-related adverse events on survival in patients with advanced non-small cell lung cancer treated with nivolumab: long-term outcomes from a multi-institutional analysis. J Cancer Res Clin Oncol 145, 479-485, doi:10.1007/s00432-018-2805-3 (2019).

31. Mushti, S. L., Mulkey, F. \& Sridhara, R. Evaluation of Overall Response Rate and Progression-Free Survival as Potential Surrogate Endpoints for Overall Survival in Immunotherapy Trials. Clin Cancer Res 24, 2268-2275, doi:10.1158/1078-0432.CCR-17-1902 (2018).

32. Tan, A., Porcher, R., Crequit, P., Ravaud, P. \& Dechartres, A. Differences in Treatment Effect Size Between Overall Survival and Progression-Free Survival in Immunotherapy Trials: A Meta-Epidemiologic Study of Trials With Results Posted at ClinicalTrials.gov. J Clin Onco/ 35, 1686-1694, doi:10.1200/JCO.2016.71.2109 (2017).

33. Reck, M. et al. Updated Analysis of KEYNOTE-024: Pembrolizumab Versus Platinum-Based Chemotherapy for Advanced Non-Small-Cell Lung Cancer With PD-L1 Tumor Proportion Score of 50\% or Greater. J Clin Onco/ 37, 537-546, doi:10.1200/JCO.18.00149 (2019).

34. Antonia, S. J. et al. Four-year survival with nivolumab in patients with previously treated advanced non-small-cell lung cancer: a pooled analysis. Lancet Oncol 20, 1395-1408, doi:10.1016/S1470-2045(19)30407-3 (2019). 
REAL-WORLD OUTCOMES VS. CLINICAL TRIAL RESULTS OF ICIS IN STAGE IV NSCLC 135 


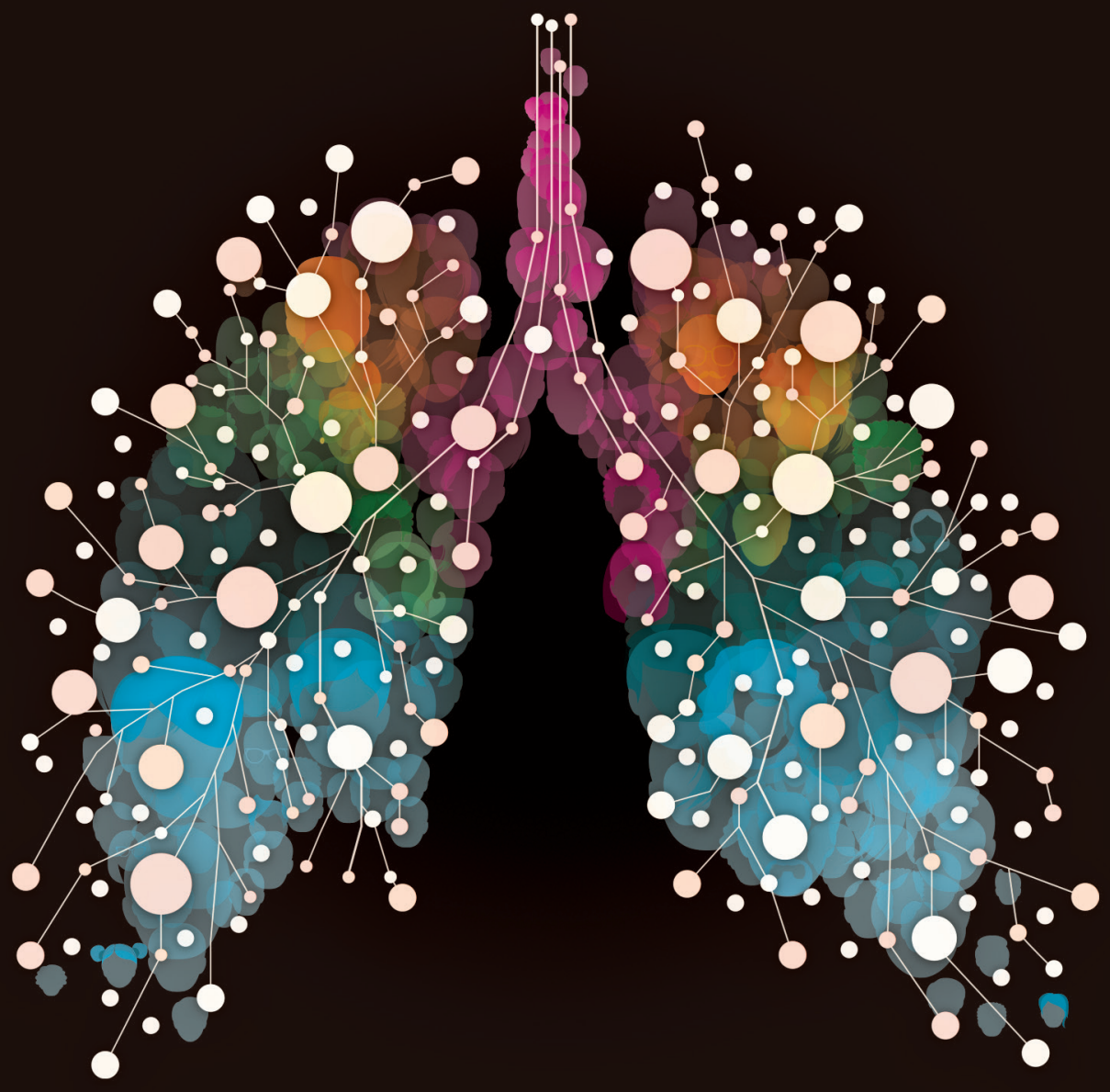


PART II

Real-world outcomes SCLC 



\section{Chapter 6}

Real-world treatment patterns and outcomes of patients with extensive disease small cell lung cancer

Christine M. Cramer-van der Welle, Franz M.N.H. Schramel, Arvid S. van Leeuwen, Harry J.M. Groen, Ewoudt M.W. van de Garde, for the Santeon SCLC Study Group 


\section{ABSTRACT}

Objective: Clinical outcome data on patients with extensive disease small cell lung cancer (ED SCLC) treated in routine practice is limited. The aim of this retrospective study is to present data on treatment patterns and survival in an unselected patient population with ED SCLC.

Methods: All patients diagnosed with ED SCLC between 2008 and 2014 in six Dutch large teaching hospitals (Santeon network) were included. We collected data on patient characteristics, systemic treatments, overall survival (OS), dose reductions ( $<80 \%$ of initial dose), and early discontinuation (<4 cycles).

Results: From 792 diagnosed patients, 568 (72\%) started with first-line treatment. Of these patients, 41\% received second-line treatment. Only 68 patients received third-line treatment. For all treated patients, the mean age was 66 years. The majority (72\%) had a performance status (ECOG) of 0 or 1 at diagnosis. Median OS of treated patients was 7.4 months. Of all patients with first-line treatment, $26 \%$ received $<4$ cycles and dose reductions were observed in 29\%.

Conclusion: After first-line systemic treatment in ED SCLC the fraction of patients receiving subsequent lines of treatment is rapidly decreasing. This information is necessary as background for evaluation of the added value of future drugs under study for ED SCLC. 


\section{INTRODUCTION}

Of all patients diagnosed with lung cancer, small cell lung cancer (SCLC) represents 13\% of the cases in the Netherlands [1]. SCLC is an aggressive tumor which is frequently metastasized at time of diagnosis, and therefore the majority of patients is diagnosed with extensive disease (ED) $[2,3]$. The median survival for patients without systemic therapy has been reported to be 2 to 4 months $[3,4]$.

Several studies have reported an improvement in survival after the introduction of chemotherapy in the 1970s [5]. Despite numerous clinical trials, systemic treatment for patients with SCLC has not changed significantly in the past several decades $[6,7]$. The standard of care in Europe for newly diagnosed ED SCLC is a platinum-based chemotherapy with etoposide [8]. In case of chemotherapy-resistant disease a second-line treatment with topotecan can be started [8,9].

These treatment standards are based on clinical trial data, which often have excluded patients with a worse performance status, comorbidities, and high age. Therefore, the question remains if patients with ED SCLC in daily practice show the same outcomes as those in clinical trials. The recent German TLK study showed that platinum-based combination chemotherapies accounted for $93 \%$ of all first-line treatments in patients with ED SCLC [10]. At least 50\% of the patients received a second-line treatment and 22\% a third-line treatment. Regarding survival time, this study concluded similarity with data from previously randomized trials. A limitation is that they started with patients receiving systemic treatment thus not being able to report on the percentage of best supportive care only after diagnosis.

A recent systematic literature review on real-world effectiveness of SCLC treatments by Povsic et al. also emphasized the limited number of treatment options in SCLC and lack of good quality real-world data about outcomes [11]. The aim of the present study is to provide good quality data on treatment patterns, including best supportive care, and corresponding survival times in an unselected patient population with ED SCLC.

\section{PATIENTS AND METHODS}

\section{Data source and study participants}

This study was performed within a network of seven large (non-university) teaching hospitals in the Netherlands, named Santeon, which serves more than 12\% of the Dutch patient population. We used the Santeon Care for Outcome (CfO) registry for identifying all patients diagnosed with ED SCLC between 2008 and 2014, and for collecting patient characteristics. Data on applied systemic pharmacotherapy for ED SCLC was derived from individual patient files. Furthermore, the Santeon Farmadatabase (SFD) was used for validation and collection of additional detailed data about systemic treatments. More details on the CfO registry and SFD can be found elsewhere [12-14]. 
Study data were collected and managed using REDCap electronic data capture tools hosted at St. Antonius Hospital, Utrecht/Nieuwegein, the Netherlands [15]. This study was approved by a medical research ethics committee (CMO registration number 2018-4338), with need for informed consent being waived because of the retrospective nature of the study and anonymous handling of data.

\section{Patient characteristics and systemic treatment per patient}

The following patient characteristics were collected from the CfO: date of diagnosis, age at diagnosis, gender, ECOG performance status (PS), separate comorbidities (to calculate Charlson Comorbidity Index (CCI)), and date of death.

For every patient, all systemic treatments were extracted from both the individual patient files and the prescription data recorded in the SFD, including start and stop dates, number of cycles and dose, and whether it was first, second or further line of treatment. The initial systemic therapy following date of diagnosis was defined as first-line treatment. Switches from cisplatin to carboplatin due to toxicity were considered the same line. Second-line treatment was defined as systemic treatment applied after completion or discontinuation because of disease progression of first-line treatment. Re-induction treatment (systemic treatment with the same or similar regimen as administered in the previous line, $\geq 90$ days after finishing first-line treatment) for chemo-sensitive patients was considered a subsequent line of treatment.

\section{Real-world treatment outcomes}

The overall survival (OS) for all treated patients was calculated using time between start date of systemic treatment and date of death. For patients receiving best supportive care, date of diagnosis was used to calculate OS. Patients still alive at January 31, 2018 (date of update from Personal Records Database (BRP)) were given this end of follow-up date as imputed date of death $(n=7)$. Furthermore, progression-free survival (PFS) was calculated as time between start date of systemic treatment and date of progression when noted, or date of death in absence of acknowledged progression from the individual patient files. As proxies for toxicity, the percentage of patients with dose reductions ( $<80 \%$ of the initial dose), early discontinuation (at least one cycle less than planned for that regimen) and/or switches were assessed within lines of treatment.

\section{Statistical analysis}

Statistical Software (SPSS version 24 for Windows; IBM) was used for statistical analysis. In case of continuous data mean \pm SD or median (range) was given, categorical data were analysed using chi-square and continuous data using t tests and one-way ANOVA when appropriate. 


\section{RESULTS}

\section{Patient characteristics}

We identified 792 patients diagnosed with ED SCLC in the period 2008-2014, of which 568 (72\%) started with first-line treatment. A combination therapy with cisplatin or carboplatin and etoposide is the most applied first-line systemic treatment ( $96 \%$ of all treated patients). Table 1 presents the baseline patient characteristics per systemic first-line treatment regimen, for all treated patients as a whole, and for patients who received best supportive care. For all treated patients, the mean age at diagnosis was 66 years; $45 \%$ were female. The majority of patients (72\%) had an ECOG PS 0-1 at time of diagnosis and comorbidities were present in $57 \%$ of all patients $(\mathrm{CCl}>0)$. For all patients with best supportive care, 87 patients (39\%) had an ECOG PS 0-1 at time of diagnosis, but nevertheless did not start first-line treatment for various reasons, for example, personal preferences or very rapid progress of disease. Table A1 (Appendix) presents the baseline characteristics for all treated patients on start of first, second or third-line treatment.

\section{Treatment patterns}

Figure 1 shows the different treatment patterns of all patients towards best supportive care (BSC). Of all patients treated with first-line treatment, $41 \%(n=231)$ received second-line treatment, of which $24 \%$ received topotecan. Apart from re-induction chemotherapy with platinum-etoposide (48\%), taxane-based regimens were the most commonly used type of regimens (19\%) in second-line. Only 68 patients (12\%) received third-line treatment. In third-line, topotecan was most commonly applied (32\%), followed by taxane-based regimens (31\%). Twelve patients (18\%) received re-induction treatment with a platinum-etoposide regimen. In fourth-line, topotecan was applied in half of the patients. Two patients subsequently received fifth-line systemic treatment.

\section{Outcomes}

Median PFS and OS for all treated patients were 5.8 and 7.4 months, respectively. Median PFS and OS per line of systemic treatment were shown in Table 2. Median OS for patients with best supportive care $(n=224)$ was 0.9 months.

There was no difference in median OS for treated patients depending on year of diagnosis $(p=0.868)$. Furthermore, no significant difference was found in median OS for patients with second-line re-induction chemotherapy (platinum-etoposide) depending on time to progression from end date of first-line therapy $(<6$ months $(n=54)$ versus $\geq 6$ months $(n=55))$ : median OS from start date of second-line therapy was 5.6 and 7.0 months, respectively $(p=0.067)$. 


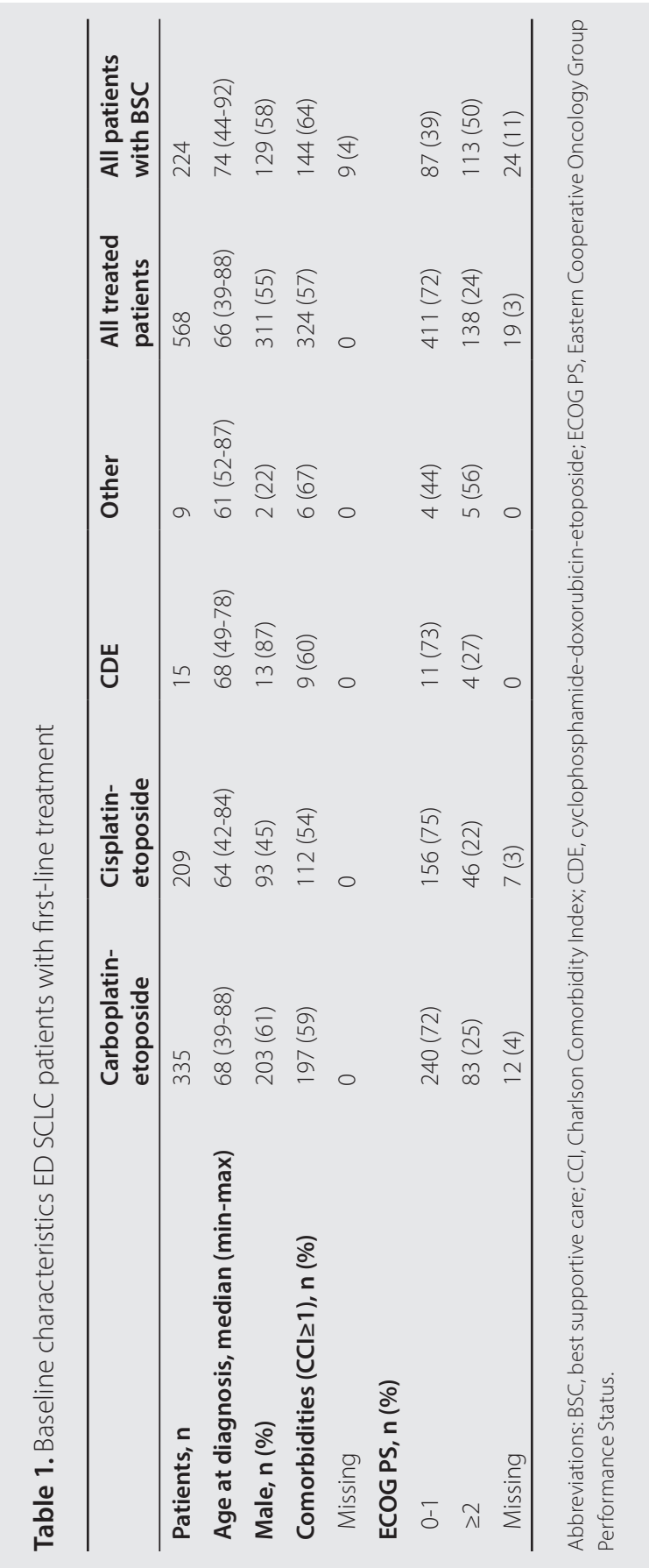




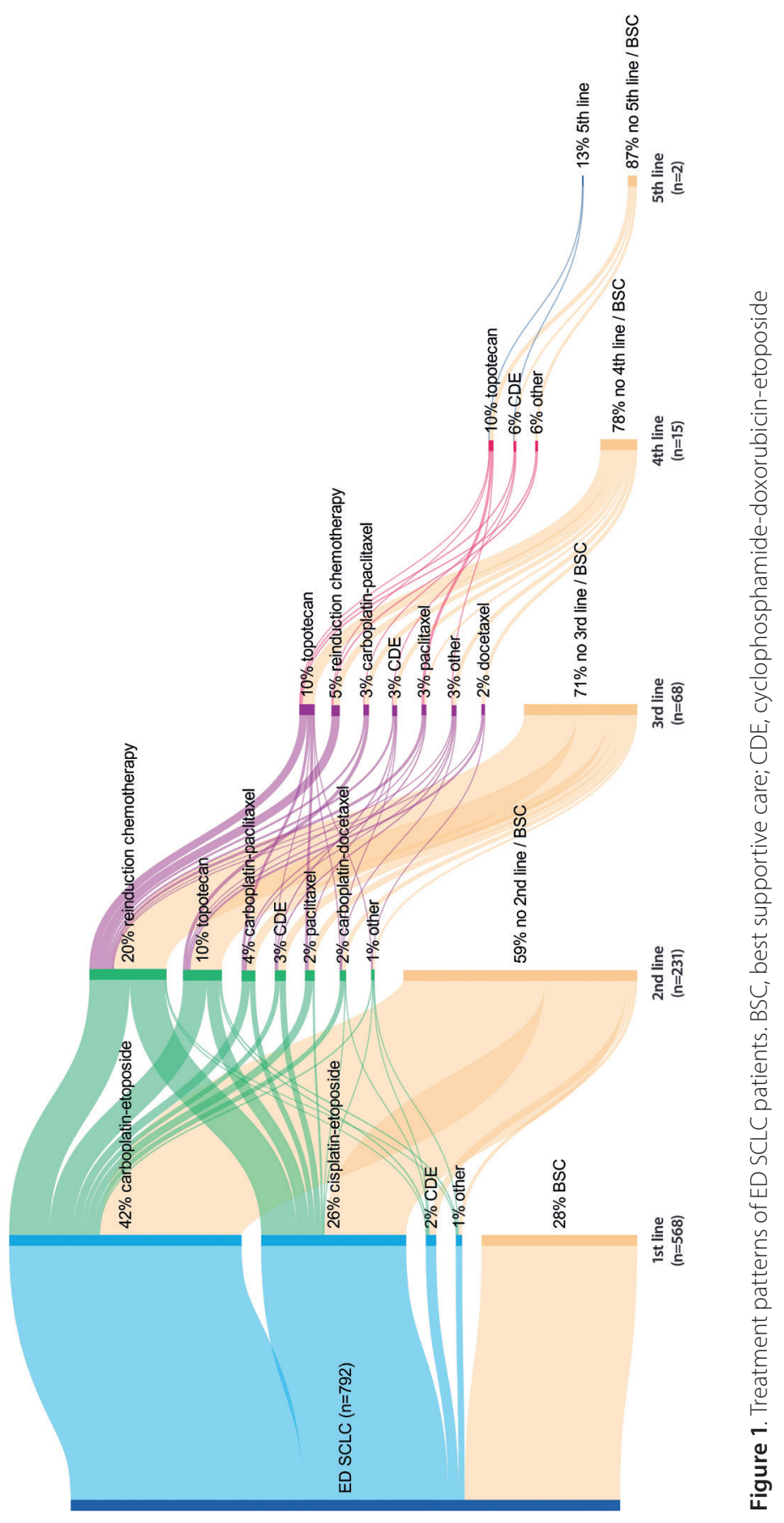


Table 2. PFS and OS of treated patients from start date of corresponding line

\begin{tabular}{|c|c|c|c|c|c|}
\hline & $\begin{array}{l}1^{\text {st }} \text { line } \\
(n=568 \text { treated) }\end{array}$ & $\begin{array}{l}2^{\text {nd }} \text { line } \\
(n=231)\end{array}$ & $\begin{array}{l}3^{\text {rd line }} \\
(n=68)\end{array}$ & $\begin{array}{l}4^{\text {th }} \text { line } \\
(n=15)\end{array}$ & $\begin{array}{l}5^{\text {th }} \text { line } \\
(n=2)\end{array}$ \\
\hline Median PFS (months) & 5.8 & 3.4 & 3.4 & 3.7 & 4.7 \\
\hline Median OS (months) & 7.4 & 4.6 & 3.7 & 5.1 & 6.0 \\
\hline
\end{tabular}

Abbreviations: OS, overall survival; PFS, progression-free survival.

Table 3. Toxicity in patients with first-line treatment

\begin{tabular}{llll}
\hline Regimen & $\begin{array}{l}\text { Dose } \\
\text { reduction (\%) }\end{array}$ & $\begin{array}{l}\text { Early } \\
\text { discontinuation (\%) }\end{array}$ & $\begin{array}{l}\text { Switch } \\
\text { (\%) }\end{array}$ \\
\hline Carboplatin-etoposide $(n=335)$ & 26 & 27 & 2 \\
Cisplatin-etoposide $(n=209)$ & 35 & 23 & 18 \\
CDE $(n=15)$ & 40 & 33 & 7 \\
Other $(n=9)$ & 22 & 44 & 22 \\
Overall & $\mathbf{2 9}$ & $\mathbf{2 6}$ & $\mathbf{8}$ \\
\hline
\end{tabular}

Abbreviation: CDE, cyclophosphamide-doxorubicin-etoposide

Of all patients with first-line treatment, dose reductions were present in $29 \%$, early discontinuation in 26\%, and switches in $8 \%$ of the patients (Table 3). Multivariate analysis showed a statistically significant association of age, ECOG PS, and CCI with the occurrence of one or more proxies for toxicity ( $p=0.046, p=0.004$ and $p=0.017$, respectively).

\section{DISCUSSION}

This study showed that after first-line systemic treatment in ED SCLC the fraction of patients receiving subsequent lines is rapidly decreasing. From 792 diagnosed patients, $72 \%$ started with first-line treatment. Of these patients, $41 \%$ received second-line treatment and only 12\% third-line treatment. The median OS from start of first-line treatment is 7.4 months. Combination therapy with platinum-etoposide is the most applied first-line systemic treatment (96\% of all treated patients).

This is one of the few studies that provide a complete overview of treatment patterns and corresponding outcomes for an unselected population of patients with ED SCLC. To the 
best of our knowledge, the German TLK study of Steffens et al. [10] and the cohort study of Tendler et al. [16] are the only other recent studies including such data. Our findings are in line with Steffens et al. with regard to low percentages of patients receiving a second and third-line. Furthermore, we found a comparable percentage of platinum-based combination chemotherapy as first-line treatment. In addition to Steffens et al., our study revealed that $28 \%$ of all patients diagnosed with ED SCLC received best supportive care only. This means that the statement of Steffens et al. that their results may not be generalised to the small group of patients not receiving any systemic treatment concerns more than a quarter of all patients diagnosed with ED SCLC [10].

In contrast to the German TLK study, we found a lower median OS from first-line treatment (7.4 months, compared to 10.7 as found by Steffens et al.), whereas Tendler et al. reported a median OS of 7.0 months, which is in line with our findings. These differences may be related to differences in cohort selection. The TLK study included patients who received at least one palliative line of treatment, whereas in our study and in the study of Tendler et al. all patients who started first-line treatment were included (i.e. at least one cycle of treatment). The possibility exist that some patients have not made it into the Steffens et al. cohort because of early discontinuation of treatment due to rapid progression or toxicity. The remaining patients do have a better prognosis which is reflected in a longer median survival. Furthermore, it is not inconceivable that the process of prospective enrolment of patients in the TLK study might have caused selection bias by missing patients with poor prognosis. For example, Steffens et al. reported 39\% of all patients with CCl $\geq 1$ and $17 \%$ with ECOG PS $\geq 2$, compared to $57 \%$ and $24 \%$ in our population treated with first-line systemic treatment. Another contrast with the German TLK Study is that in second-line regimens with paclitaxel or docetaxel were applied more often in our population (19\% vs. $<3 \%)$. This is in contrast with the clinical practice guidelines in the US and Europe recommending topotecan for patients eligible for second-line treatment in case of chemotherapy-resistant disease $[8,9,17]$. Topotecan is the only second-line drug approved by the FDA (US Food and Drug Administration). One reason for this divergence from guidelines could be Netherlands based research of Groen et al. who observed a high response rate and mild toxicity of second-line carboplatin-paclitaxel in CDE-resistant SCLC patients [18].

A strength of this study is that it is based on a large unselected real-world population of patients diagnosed with ED SCLC, geographically spread over the Netherlands. In addition, this study is based on high-resolution data with a very low number of missing values.

The limitations of this study primarily relate to the retrospective nature of the data collection and the time frame under study (2008-2014), which can possibly affect the generalisability of our findings to present daily clinical practice. On the other hand, we captured a time frame of $>7$ years without any effect of calendar year on the findings. Recently, improved OS and PFS were reported with the addition of atezolizumab [19] or durvalumab [20] to chemotherapy in first-line compared to chemotherapy alone. The 
latest National Comprehensive Cancer Network (NCCN) guidelines included these chemoimmunotherapy regimens as a first-line option for ED SCLC patients [21]. Future studies capturing more recent years are needed to discover how these new treatment options find their way in routine practice. Furthermore, due to the retrospective nature of the data collection, some data items were not captured, e.g. site of metastases and the use of radiotherapy. Consequently, a statement about the impact of radiotherapy and the localization of metastases on treatment prognosis was not possible.

Our findings are of interest when designing clinical trials for future drugs under study for ED SCLC. We showed that the number of patients who will be eligible for a second and/or third-line of systemic therapy is limited in real-world. Furthermore, insight in treatment patterns is helpful to payers when considering the budget impact (e.g. expected patient volume) of novel treatment options when they come available.

In conclusion, after first-line systemic treatment in ED SCLC the fraction of patients receiving subsequent lines of treatment is rapidly decreasing. This information is necessary as background for the evaluation of the added value of future drugs under study for ED SCLC.

\section{ACKNOWLEDGEMENTS}

The authors thank M.J. Deenen and E.A.F. Haak for their efforts to complete the hospital pharmacy data. 


\section{REFERENCES}

1. Netherlands Comprehensive Cancer Organisation. www.cijfersoverkanker.nl; accessed 14-03-2019.

2. Lally BE, Urbanic JJ, Blackstock AW, Miller AA, Perry MC. Small cell lung cancer: have we made any progress over the last 25 years? Oncologist. 2007;12:1096-104.

3. Carney DN. Lung cancer--time to move on from chemotherapy. N Engl J Med. 2002;346:126-8.

4. van Meerbeeck JP, Fennell DA, De Ruysscher DK. Small-cell lung cancer. Lancet. 2011;378:1741-55.

5. Janssen-Heijnen ML, Karim-Kos HE, van der Drift MA, Groen HJ, Ho VK, Koning C, et al. Modest improvements of survival for patients with small cell lung cancer aged 45 to 59 years only, diagnosed in the Netherlands, 1989 to 2008. J Thorac Oncol. 2012;7:227-32.

6. Byers LA, Rudin CM. Small cell lung cancer: where do we go from here? Cancer. 2015;121:664-72.

7. Pietanza MC, Byers LA, Minna JD, Rudin CM. Small cell lung cancer: will recent progress lead to improved outcomes? Clin Cancer Res. 2015;21:2244-55.

8. Fruh M, De Ruysscher D, Popat S, Crino L, Peters S, Felip E, et al. Small-cell lung cancer (SCLC): ESMO Clinical Practice Guidelines for diagnosis, treatment and follow-up. Ann Oncol. 2013;24 Suppl 6:vi99-105.

9. Ardizzoni A. Topotecan in the treatment of recurrent small cell lung cancer: an update. Oncologist. 2004;9 Suppl 6:4-13.

10. Steffens CC, Elender C, Hutzschenreuter U, Dille S, Binninger A, Spring L, et al. Treatment and outcome of 432 patients with extensive-stage small cell lung cancer in first, second and third line-Results from the prospective German TLK cohort study. Lung Cancer. 2019;130:216-25.

11. Povsic M, Enstone A, Wyn R, Kornalska K, Penrod JR, Yuan Y. Real-world effectiveness and tolerability of small-cell lung cancer (SCLC) treatments: A systematic literature review (SLR). PLoS One. 2019;14:e0219622.

12. Cramer-van der Welle CM, Peters BJM, Schramel F, Klungel OH, Groen HJM, van de Garde EMW, et al. Systematic evaluation of the efficacy-effectiveness gap of systemic treatments in metastatic nonsmall cell lung cancer. Eur Respir J. 2018;52.

13. Peters BJM, Cramer-Vd Welle CM, Smit AAJ, Schramel F, van de Garde EMW, Santeon NSCLC Study Group. Trends in prescribing systemic treatment and overall survival for non-small cell lung cancer stage IIIB/IV in the Netherlands: 2008-2012. Cancer Epidemiol. 2017;51:1-6.

14. van de Garde EMW, Plouvier BC, Fleuren H, Haak EAF, Movig KLL, Deenen MJ, et al. Pharmacotherapy within a learning healthcare system: rationale for the Dutch Santeon Farmadatabase. Eur J Hosp Pharm. 2019;26:46-50.

15. Harris PA, Taylor R, Thielke R, Payne J, Gonzalez N, Conde JG. Research electronic data capture (REDCap)--a metadata-driven methodology and workflow process for providing translational research informatics support. J Biomed Inform. 2009;42:377-81.

16. Tendler S, Zhan Y, Pettersson A, Lewensohn R, Viktorsson K, Fang F, et al. Treatment patterns and survival outcomes for small-cell lung cancer patients - a Swedish single center cohort study. Acta Oncol. 2020:1-7.

17. Rudin CM, Ismaila N, Hann CL, Malhotra N, Movsas B, Norris K, et al. Treatment of Small-Cell Lung Cancer: American Society of Clinical Oncology Endorsement of the American College of Chest Physicians Guideline. J Clin Oncol. 2015;33:4106-11.

18. Groen HJ, Fokkema E, Biesma B, Kwa B, van Putten JW, Postmus PE, et al. Paclitaxel and carboplatin in the treatment of small-cell lung cancer patients resistant to cyclophosphamide, doxorubicin, and etoposide: a non-cross-resistant schedule. J Clin Oncol. 1999;17:927-32.

19. Horn L, Mansfield AS, Szczesna A, Havel L, Krzakowski M, Hochmair MJ, et al. First-Line Atezolizumab plus Chemotherapy in Extensive-Stage Small-Cell Lung Cancer. N Engl J Med. 2018;379:2220-9.

20. Paz-Ares L, Dvorkin M, Chen Y, Reinmuth N, Hotta K, Trukhin D, et al. Durvalumab plus platinum-etoposide versus platinum-etoposide in first-line treatment of extensive-stage small-cell lung cancer (CASPIAN): a randomised, controlled, open-label, phase 3 trial. Lancet. 2019;394:1929-39.

21. National Comprehensive Cancer Network ${ }^{\circledR}$. NCCN Clinical Practice Guidelines in Oncology - Small Cell Lung Cancer. Version 2.2020 - November 15, 2019. NCCN.org. 


\section{APPENDIX CHAPTER 6}

Table A1. Baseline characteristics ED SCLC patients on start of first, second, or third-line treatment

\begin{tabular}{llll}
\hline & $\begin{array}{l}\text { All treated } \\
\text { patients } \\
\text { in first-line }\end{array}$ & $\begin{array}{l}\text { All treated } \\
\text { patients } \\
\text { in second- } \\
\text { line }\end{array}$ & $\begin{array}{l}\text { All treated } \\
\text { patients } \\
\text { in third-line }\end{array}$ \\
\hline Patients, $\mathbf{n}(\%)$ & 568 & 231 & 68 \\
Age at diagnosis, median (min-max) & $66(39-88)$ & $64(39-83)$ & $62(46-81)$ \\
Male, $\mathbf{n}(\%)$ & $311(55)$ & $118(51)$ & $35(51)$ \\
Comorbidities (CCl $\geq 1), \mathbf{n}(\%)$ & $324(57)$ & $121(52)$ & $33(49)$ \\
Missing & 0 & 0 & 0 \\
ECOG PS, $\mathbf{n}(\%)$ & & $182(79)$ & $58(85)$ \\
$0-1$ & $411(72)$ & $42(18)$ & $9(13)$ \\
$\geq 2$ & $138(24)$ & $7(3)$ & $1(1)$ \\
\hline Missing & $19(3)$ & & \\
\hline
\end{tabular}

Abbreviations: BSC, best supportive care; CCI, Charlson Comorbidity Index; CDE, cyclophosphamide-doxorubicinetoposide; ECOG PS, Eastern Cooperative Oncology Group Performance Status. 
REAL-WORLD TREATMENT PATTERNS AND OUTCOMES OF PATIENTS WITH ED SCLC | 151 



\section{Chapter 7}

Systematic evaluation of the efficacyeffectiveness gap of systemic treatments in extensive disease small cell lung cancer

Christine M. Cramer - van der Welle, Franz M.N.H. Schramel, Bas J.M. Peters, John W.G. van Putten, Olaf H. Klungel, Harry J.M. Groen, Ewoudt M.W. van de Garde, for the Santeon SCLC Study Group

Pharmacoepidemiol Drug Saf. 2020 Dec 9 


\section{ABSTRACT}

Purpose: The aim of this study is to assess how clinical outcomes in real-world (effectiveness) correspond to the outcomes in clinical trials (efficacy) of systemic treatments for extensive disease small cell lung cancer (ED SCLC).

Methods: All patients diagnosed with ED SCLC between 2008 and 2014 in six Dutch large teaching hospitals (Santeon network) were identified and followed-up from date of diagnosis until death or end of data collection. For every patient, an efficacy-effectiveness factor (EE factor) was calculated by dividing individual patients' overall survival (OS) by the pooled median OS assessed from clinical trials with the respective treatment.

Results: From 792 diagnosed patients, 568 (72\%) started with first-line treatment. Overall, the median EE factor was 0.79 ( $p<0.001$ from 1.00). Poor performance status ( $E C O G \geq 2$ ) and a higher age at diagnosis (age $\geq 65$ years) were independent predictors for a lower EE factor. The EE gap was $43 \%$ in patients with both age $\geq 65$ years and ECOG $\geq 2$ (EE factor 0.57). The mean age and the proportion of patients with $E C O G \geq 2$ in real-world were different from those in clinical trials (mean age of 66 versus 62 years, and ECOG $\geq 2$ 25\% versus $17 \%$; both $\mathrm{p}<0.001$ ).

Conclusion: OS of patients with ED SCLC treated with systemic therapy in real-world practice is $21 \%$ shorter than for patients included in trials. Age at diagnosis and performance status partly explain this gap. 


\section{INTRODUCTION}

Small cell lung cancer (SCLC) is characterized by its rapid growth, high response rate to chemotherapy and early relapse in patients with metastatic disease. SCLC represents 13\% of all lung cancer diagnosis in the Netherlands ${ }^{2}$ and other countries in the Western world.? The majority of patients is diagnosed with extensive disease (ED)3,4, with limited treatment options and a median overall survival (OS) of less than 10 months when treated with chemotherapy. Platinum-based combination chemotherapy is standard of care in the United States and Europe as first-line treatment; for second-line treatment re-induction or topotecan can be started.1,5-7

The recommendations in the treatment standards mentioned above are conventionally based on clinical trial data, in which patient populations are studied that are not necessarily a reflection of the general population seen in clinical practice. ${ }^{8}$ Important patient characteristics predictive for treatment response are often underrepresented in clinical trial populations. Although data from clinical trials provide important evidence of clinical efficacy, the effectiveness in real-world is largely unknown.

In a previous study in non-small cell lung cancer (NSCLC), we found that survival of patients treated with systemic therapy in real-world practice is almost one quarter shorter than for patients included in clinical trials. ${ }^{9}$ A recent systematic literature review on real-world effectiveness of SCLC treatments by Povsic et al. suggested that such an efficacy-effectiveness gap is also present in SCLC. 8 However, they also reported a lack of good quality real-world data about outcomes and emphasize the need to examine this further. For example, individual patient data level studies exploring characteristics associated with a possible efficacy-effectiveness gap are missing.

The aim of the present study is to assess the difference between outcomes of systemic treatments for ED SCLC in clinical trials and in real-world practice in a large nationwide cohort of patients with ED SCLC, and to search for explanatory factors that may explain a gap.

\section{METHODS}

\section{Data source and study participants}

This cohort study was conducted using clinical data originating from the Santeon network of seven large (non-university) teaching hospitals in the Netherlands, which serves more than $12 \%$ of the Dutch patient population. We used the Santeon Care for Outcome (CfO) registry for identifying all patients diagnosed with ED SCLC between 2008 and 2014, and for collecting patient characteristics. Data on systemic treatment for ED SCLC was derived from individual patient files. Furthermore, the Santeon Farmadatabase (SFD) was used for validation and collecting additional detailed data about systemic treatments. More details on the CfO registry and SFD can be found elsewhere. ${ }^{9-11}$ 
Study data were collected and managed using REDCap electronic data capture tools 12 hosted at St. Antonius Hospital, Utrecht/Nieuwegein, the Netherlands. This study was approved by a medical research ethics committee (CMO registration number 2018-4338), with need for informed consent being waived because of the retrospective nature of the study and anonymous handling of data.

\section{Patient characteristics and systemic treatment per patient}

From the CfO registry, we collected the following patient characteristics: date of diagnosis, age at diagnosis, gender, ECOG performance status (PS), separate comorbidities (to calculate Charlson Comorbidity Index (CCI)), and date of death.

Systemic treatment(s) per patient were extracted from both the individual patient files and the prescription data recorded in the SFD, including start and stop dates, number of cycles and dose, and whether it was first, second or further line of treatment. First-line treatment was defined as the initial systemic therapy following date of diagnosis. Switches to another regimen (e.g. from cisplatin-etoposide to carboplatin-etoposide) due to toxicity were considered the same line. Second-line treatment was defined as systemic treatment applied after completion of first-line treatment, or discontinuation of first-line treatment because of disease progression. Re-induction treatment (systemic treatment with the same or similar regimen as administered in the previous line, $\geq 90$ days after finishing first-line treatment) for chemo-sensitive patients was considered a subsequent line of treatment.

\section{Systematic literature review for reference outcomes}

For all first-line treatment regimens in the study population (except rarely applied regimens $(<2 \%)$ which were coded as 'other'), a systematic literature search (up to September 12, 2018) and meta-analysis were conducted to obtain a (pooled) clinical trial (efficacy) result. Exact details of the search in PubMed, Embase, and CENTRAL (Cochrane library) are provided in Appendix 1. Duplicates were identified and removed using RefWorks (RefWorks Web Based Bibliographic Management Software, ProQuest LLC). An article was included if all the following criteria were met: a) patients diagnosed with SCLC; b) main article of a phase III randomized trial; c) intervention under study is one of the first-line regimens identified in our data; d) patients with extensive / stage IV disease; and e) OS as outcome (with data about distribution of survival times). Criteria for exclusion of articles, the eligibility screening of articles, and the method to determine the reference outcome per regimen is described in further detail by Cramer et al. ${ }^{9}$ Appendix 2 provides in more detail per regimen the yield of the systematic review and the meta-analysis data.

\section{Real-world treatment outcomes}

For every individual patient, an OS was calculated using time between start date of systemic treatment and date of death. Patients still alive at January 31, 2018 (date of update 
from Personal Records Database (BRP)) were censored and given this end of follow-up date as imputed date of death $(n=7)$. An efficacy-effectiveness factor (EE factor) was calculated for every patient by dividing the individual real-world OS by the reference outcome (OS) from the corresponding first-line regimen. Toxicity was assessed using percentages of patients with dose reductions ( $<80 \%$ of the initial dose), early discontinuation (at least one cycle less than planned for that regimen) and/or treatment switches within lines of treatment as proxy.

\section{Statistical analysis}

Statistical Software (SPSS version 24 for Windows; IBM, Armonk, New York) was used for statistical analysis. In case of continuous data mean \pm SD or median (range) was given, categorical data was analysed using chi-square and continuous data using t-tests and one-way ANOVA when appropriate.

To assess the existence of a significant EE gap overall and per regimen, the distribution of the calculated EE factors was tested relative to 1.0 using the Wilcoxon signed-rank test. Next, a multivariable linear regression analysis was applied after log-transformation of the EE factor to study the association between patient and treatment characteristics and the magnitude of the EE gap. First, an explanatory analysis was performed to study patient characteristics at diagnosis (age, gender, Charlson comorbidity index (CCI), ECOG performance status (PS), histology and year of diagnosis) as potential prognostic factors. In this analysis, missing values were imputed by single stochastic regression imputation (single run with all available characteristics in the model). Second, we examined whether identified determinants were differently distributed between our population and the clinical trial data to support a potential causal relation. The latter was done by standard descriptive statistics. Third, a multivariable analysis was conducted with toxicity and dose intensity related treatment factors (dose reduction, early discontinuation, switches, and no subsequent line of chemotherapy) as possible associated factors with patient characteristics. Finally, to assess the robustness of our main analysis regarding the presence and significance of the EE gap, a sensitivity analysis was done with calculating the main outcome (OS in real-world) not from start of treatment, but based on date of diagnosis.

\section{RESULTS}

From 792 diagnosed patients, 568 (72\%) started with first-line treatment. Table 1 presents the baseline patient characteristics per systemic first-line treatment regimen. At diagnosis, the mean age of all treated patients was 66 years, 72\% had an ECOG PS 0-1 (3\% missing data), and comorbidities ( $\mathrm{CCl}>0$ ) were present in $57 \%$ of the patients. Overall, three regimens (carboplatin-etoposide, cisplatin-etoposide, and cyclophosphamide-doxorubicin-etoposide (CDE)) were responsible for $98 \%$ of the variety in applied first-line 
treatments ( $n=559$ patients). Dose densities were according to the Dutch guidelines. ${ }^{2}$ Table 2 outlines the real-world OS, reference OS from clinical trials (range of inclusion periods from 1985 to 2015), and EE factor for these three first-line regimens. For all regimens, the median OS in real-world is shorter than the clinical trial reference median OS. Overall, the distribution of the EE factor is significantly different from a hypothesized median of 1.00 (median EE factor of $0.79 ; 95 \% \mathrm{Cl} 0.68-0.84 ; \mathrm{p}<0.001$ ), and the median EE factor is $<1.00$ for all individual treatment regimens (Table 2).

Table 1. Baseline characteristics ED SCLC patients with first-line treatment

\begin{tabular}{|c|c|c|c|c|c|}
\hline & $\begin{array}{l}\text { Carboplatin- } \\
\text { etoposide }\end{array}$ & $\begin{array}{l}\text { Cisplatin- } \\
\text { etoposide }\end{array}$ & $\mathrm{CDE}$ & Other & $\begin{array}{c}\text { All treated } \\
\text { patients }\end{array}$ \\
\hline Patients, n & 335 & 209 & 15 & 9 & 568 \\
\hline $\begin{array}{l}\text { Age at diagnosis, } \\
\text { median (min-max) }\end{array}$ & 68 (39-88) & $64(42-84)$ & $68(49-78)$ & $61(52-87)$ & $66(39-88)$ \\
\hline Male, n (\%) & $203(61)$ & $93(45)$ & $13(87)$ & $2(22)$ & $311(55)$ \\
\hline $\begin{array}{l}\text { Comorbidities }(\mathrm{CCl} \geq 1) \text {, } \\
\text { n (\%) }\end{array}$ & $197(59)$ & $112(54)$ & $9(60)$ & $6(67)$ & $324(57)$ \\
\hline \multicolumn{6}{|l|}{ ECOG PS, n (\%) } \\
\hline $0-1$ & $240(72)$ & $156(75)$ & $11(73)$ & $4(44)$ & $411(72)$ \\
\hline$\geq 2$ & $83(25)$ & $46(22)$ & $4(27)$ & $5(56)$ & $138(24)$ \\
\hline Missing & $12(4)$ & $7(3)$ & 0 & 0 & $19(3)$ \\
\hline
\end{tabular}

BSC, best supportive care; CCl, Charlson Comorbidity Index; CDE, cyclophosphamide-doxorubicin-etoposide; ECOG PS, Eastern Cooperative Oncology Group Performance Status; ED SCLC, extensive disease small cell lung cancer.

The multivariable regression analysis showed that age at diagnosis ( $<\geq 65$ years) and a patients' ECOG PS $(<\geq 2)$ were significantly associated with the magnitude of the EE factor (Table 3). The negative B-values indicate a larger EE gap for patients aged $\geq 65$ and a higher/worse ECOG PS. The EE gap was $9 \%$ in patients $<65$ years (EE factor $0.91, p=0.045$ ) and $28 \%$ in patients with age $\geq 65$ years (EE factor $0.72, p<0.001 ; n=319$ ). Furthermore, the EE gap in patients with ECOG 0-1 was 14\% (EE factor 0.86, p<0.001) and 38\% in patients with $E C O G \geq 2$ (EE factor $0.62, p<0.001 ; n=141$ ). Both the mean age of patients in real-world and the proportion of patients with $E C O G \geq 2$ in real-world were different from those in clinical trials (66 vs 62 years, and 25\% vs 17\%, respectively; both $p<0.001$ ). For patients aged $\geq 65$ years and $E C O G \geq 2(n=104)$, the EE gap was $43 \%$. 
Table 2. OS and EE factor per first-line regimen

\begin{tabular}{lcccc}
\hline & $\begin{array}{c}\text { Patients } \\
(\mathbf{n})\end{array}$ & $\begin{array}{c}\text { Median OS } \\
\text { real-world }\end{array}$ & $\begin{array}{c}\text { Median OS } \\
\text { clinical trials }\end{array}$ & $\begin{array}{c}\text { Median EE factor } \\
(\mathbf{9 5 \%} \text { CI) }\end{array}$ \\
\hline Carboplatin-etoposide & 335 & $7.23^{\mathrm{b}}$ & $9.48^{14-22}$ & $0.76 \mathrm{c}(0.68-0.84)$ \\
Cisplatin-etoposide & 209 & $8.18^{\mathrm{b}}$ & $9.4318,23-42$ & $0.87 \mathrm{C}(0.76-0.97)$ \\
CDE & 15 & 5.62 & $7.29^{43,44}$ & $0.77(0.19-1.28)$ \\
Total & $\mathbf{5 5 9}$ & $\mathbf{7 . 4 3 ^ { \mathrm { b } }}$ & $\mathbf{9 . 3 2}$ & $\mathbf{0 . 7 9 c}(\mathbf{0 . 7 4 - 0 . 8 6 )}$ \\
\hline
\end{tabular}

OS: overall survival (in months); CDE: cyclophosphamide-doxorubicin-etoposide.

a Calculated $95 \% \mathrm{Cl}$ hold a risk of over estimation because of not including uncertainty in the fixed reference median OS from the clinical trials.

b Significantly different ( $p$-values $<0.05$ ) from median OS clinical trials.

c Significantly different ( $p$-values $<0.05$ ) from test value 1.00 (one-sample Wilcoxon signed-rank test).

Table 3. Univariable and multivariable analysis of potential prognostic patient variables

\begin{tabular}{lcccc}
\hline Variable & \multicolumn{2}{c}{ Univariate analysis } & \multicolumn{2}{c}{ Multivariate analysis } \\
\hline & B-value & $95 \%$ Cl & B-value & $95 \%$ Cl \\
\hline Age $\geq 65$ years & -0.187 & -0.274 to -0.100 & -0.133 & -0.223 to -0.044 \\
Gender & -0.117 & -0.205 to -0.030 & -0.084 & -0.171 to 0.002 \\
ECOG PS $\geq 2$ & -0.215 & -0.314 to -0.116 & -0.172 & -0.273 to -0.072 \\
CCI $\geq 1$ & -0.112 & -0.200 to -0.025 & -0.066 & -0.154 to 0.021 \\
Year of diagnosis & 0.010 & -0.013 to 0.032 & & \\
\hline
\end{tabular}

CCl, Charlson Comorbidity Index; Cl, confidence interval; ECOG PS, Eastern Cooperative Oncology Group Performance Status.

The sensitivity analysis confirmed the robustness of our findings (data not shown). In real-world practice, the median time between date of diagnosis and start date of systemic treatment was 10 days.

Multivariable analysis on proxies for toxicity showed a significant association of both age and ECOG PS with early discontinuation (both $p<0.001$ ) and no subsequent line of chemotherapy (both $\mathrm{p}<0.001$ ). Further analysis showed that those proxies for toxicity are more prevalent in patients with $E C O G \geq 2$ and aged $\geq 65$ years (Table 4). 
Table 4. Association between proxies for toxicity and patient characteristics

\begin{tabular}{lccccccc}
\hline & \multicolumn{3}{c}{ ECOG PS } & \multicolumn{3}{c}{ Age } \\
\cline { 2 - 8 } & $\mathbf{0 - 1}$ & $\mathbf{2}$ & $\mathbf{p}$-value & $\begin{array}{c}<\mathbf{6 5} \\
\text { years }\end{array}$ & $\begin{array}{c}\mathbf{2 6 5} \\
\text { years }\end{array}$ & p-value \\
\hline Early discontinuation (<4 cycles) (\%) & 22.0 & 37.6 & $<0.001$ & 17.9 & 32.0 & $<0.001$ \\
No subsequent line(s) of treatment (\%) & 56.0 & 69.5 & 0.005 & 49.2 & 67.1 & $<0.001$ \\
\hline
\end{tabular}

ECOG PS, Eastern Cooperative Oncology Group Performance Status.

\section{DISCUSSION}

This study showed that overall the median OS of patients with ED SCLC treated with first-line systemic therapy in real-world practice is $21 \%$ shorter than for patients studied in clinical trials (EE factor $0.79, \mathrm{p}<0.001$ ).

To our knowledge, this is the first study that provides a complete overview on the efficacy-effectiveness gap for systemic treatments in a large unselected population of patients diagnosed with ED SCLC. In combination with our previous research on the EE gap in metastatic NSCLC ${ }^{9}$, this finding adds to the conclusion that the existence of a gap is a general phenomenon in patients with stage IV lung cancer, irrespective of the type of lung cancer and the systemic treatment regimen chosen.

The magnitude of the EE gap found in our study is in line with the findings of Povsic et al. in their systematic review on real-world effectiveness on SCLC treatments. ${ }^{8}$ They referred to a matched comparison analysis, which showed that the OS benefit of treatment in real-world was $16 \%$ lower than that predicted from RCT data. ${ }^{13}$ Apart from confirmation, our study also indicates potential explanatory factors. The mean age of patients diagnosed in real-world is almost five years older than in trial populations. This confirms the general thinking that trials select more fit patients with less comorbidities. The risk of comorbidity increases with age, although CCI showed no significant association with the EE factor in the multivariable regression analysis, possibly due to registration difficulties related to the retrospective nature of the data collection. Another factor is the PS of the patients. Many trials are restricted to ECOG PS 0-1 patients but SCLC patients with higher ECOG PS aim for systemic treatment as well in clinical practice because of a high response rate to systemic treatment. The present study clearly shows that OS benefit significantly drops with worse PS. Patients should be informed about this when deciding for treatment. Especially because our study also showed that earlier discontinuation and no further lines of treatment are more prevalent in patients with $\mathrm{ECOG} \geq 2$. 
Strengths of this study are the large unselected patient population diagnosed with ED SCLC in the Netherlands, providing an overview of most applied systemic treatment options and their outcomes in real-world, from a time frame of $>7$ years, which reduces the risk for bias from variations over time. In addition, this study is based on complete and precise data with a very low number of missing values (only one variable with $3 \%$ missing data).

A limitation of this study could be our approach to compare median OS between real-world and clinical trials primarily. An alternative could be a Cox proportional hazards regression, which has many advantages toward identification of characteristics possibly related to the magnitude of the EE gap (e.g. possibility to present hazard ratio's). However, this was not feasible because of the unavailability of individual patient data (IPD) from clinical trials. For the calculation of the magnitude of the EE gap, the potential bias hereof is expected to be very small because of only $n=7$ survivors at end date of follow-up (thus not being able to censor these patients). However, the absence of IPD also inhibited a multivariable Cox regression in the search for explanatory factors. Unfortunately, IPD from past clinical trials are not available in the public domain for this type of analyses.

Furthermore, a limitation could be that the time frame under study affects the generalizability of our findings to present daily clinical practice, due to the recent introduction of novel treatment options (addition of immunotherapy to chemotherapy). On the other hand, our findings show a relevant EE gap, irrespective of the chemotherapy chosen, which might also extend to chemo-immunotherapy. Future studies capturing more recent years are needed to discover the effectiveness of these new treatment options in routine practice.

In conclusion, our results show that patients with ED SCLC treated in real-world practice have a $21 \%$ shorter survival that those in clinical trials. Differences in patients' performance status and age partly explain this gap. These two factors should be acknowledged when deciding for treatment together with patients.

\section{ACKNOWLEDGEMENTS}

The authors thank M.J. Deenen and E.A.F. Haak for their efforts to complete the hospital pharmacy data. 


\section{REFERENCES}

1. Fruh M, De Ruysscher D, Popat S, et al. Small-cell lung cancer (SCLC): ESMO Clinical Practice Guidelines for diagnosis, treatment and follow-up. Ann Oncol. 2013;24 Suppl 6:vi99-105.

2. Netherlands Comprehensive Cancer Organisation (IKNL). www.cijfersoverkanker.nl. Accessed 14/03/2019.

3. Carney DN. Lung cancer--time to move on from chemotherapy. N Eng/ J Med. 2002;346(2):126-128.

4. Lally BE, Urbanic JJ, Blackstock AW, Miller AA, Perry MC. Small cell lung cancer: have we made any progress over the last 25 years? Oncologist. 2007;12(9):1096-1104.

5. Ardizzoni A. Topotecan in the treatment of recurrent small cell lung cancer: an update. Oncologist. 2004;9 Suppl 6:4-13.

6. Rudin CM, Ismaila N, Hann CL, et al. Treatment of Small-Cell Lung Cancer: American Society of Clinical Oncology Endorsement of the American College of Chest Physicians Guideline. J Clin Oncol. 2015;33(34):41064111.

7. NVALT. Kleincellig longcarcinoom. Landelijke richtlijn, Versie: 1.0. Oncoline. https://www.oncoline.nl/files/ richtlijn_pdf/790/Kleincellig\%20longcarcinoom\%201.0.pdf. Published 2011.

8. Povsic M, Enstone A, Wyn R, Kornalska K, Penrod JR, Yuan Y. Real-world effectiveness and tolerability of small-cell lung cancer (SCLC) treatments: A systematic literature review (SLR). PLoS One. 2019;14(7):e0219622.

9. Cramer-van der Welle CM, Peters BJM, Schramel F, et al. Systematic evaluation of the efficacy-effectiveness gap of systemic treatments in metastatic nonsmall cell lung cancer. Eur Respir J. 2018;52(6).

10. Peters BJM, Cramer-Vd Welle CM, Smit AAJ, Schramel F, van de Garde EMW, Santeon NSCLC Study Group. Trends in prescribing systemic treatment and overall survival for non-small cell lung cancer stage IIIB/IV in the Netherlands: 2008-2012. Cancer Epidemiol. 2017;51:1-6.

11. van de Garde EMW, Plouvier BC, Fleuren H, et al. Pharmacotherapy within a learning healthcare system: rationale for the Dutch Santeon Farmadatabase. Eur J Hosp Pharm. 2019;26(1):46-50.

12. Harris PA, Taylor R, Thielke R, Payne J, Gonzalez N, Conde JG. Research electronic data capture (REDCap)--a metadata-driven methodology and workflow process for providing translational research informatics support. J Biomed Inform. 2009;42(2):377-381.

13. Lakdawalla DN, Shafrin J, Hou N, et al. Predicting Real-World Effectiveness of Cancer Therapies Using Overall Survival and Progression-Free Survival from Clinical Trials: Empirical Evidence for the ASCO Value Framework. Value Health. 2017;20(7):866-875.

14. Heigener DF, Manegold C, Jager E, Saal JG, Zuna I, Gatzemeier U. Multicenter randomized open-label phase III study comparing efficacy, safety, and tolerability of conventional carboplatin plus etoposide versus doseintensified carboplatin plus etoposide plus lenograstim in small-cell lung cancer in "extensive disease" stage. Am J Clin Oncol. 2009;32(1):61-64.

15. Hermes A, Bergman B, Bremnes R, et al. Irinotecan plus carboplatin versus oral etoposide plus carboplatin in extensive small-cell lung cancer: a randomized phase III trial. J Clin Oncol. 2008;26(26):4261-4267.

16. Jalal SI, Lavin P, Lo G, Lebel F, Einhorn L. Carboplatin and Etoposide With or Without Palifosfamide in Untreated Extensive-Stage Small-Cell Lung Cancer: A Multicenter, Adaptive, Randomized Phase III Study (MATISSE). J Clin Oncol. 2017;35(23):2619-2623

17. Lee SM, Woll PJ, Rudd R, et al. Anti-angiogenic therapy using thalidomide combined with chemotherapy in small cell lung cancer: a randomized, double-blind, placebo-controlled trial. J Nat/Cancer/nst.2009;101(15):10491057.

18. Okamoto H, Watanabe K, Kunikane H, et al. Randomised phase III trial of carboplatin plus etoposide vs split doses of cisplatin plus etoposide in elderly or poor-risk patients with extensive disease small-cell lung cancer: JCOG 9702. Br J Cancer. 2007:97(2):162-169.

19. Schmittel A, Sebastian M, Fischer von Weikersthal L, et al. A German multicenter, randomized phase III trial comparing irinotecan-carboplatin with etoposide-carboplatin as first-line therapy for extensive-disease small-cell lung cancer. Ann Oncol. 2011;22(8):1798-1804.

20. Seckl MJ, Ottensmeier CH, Cullen M, et al. Multicenter, Phase III, Randomized, Double-Blind, Placebo-Controlled Trial of Pravastatin Added to First-Line Standard Chemotherapy in Small-Cell Lung Cancer (LUNGSTAR). J Clin Oncol. 2017;35(14):1506-1514. 
21. Sekine I, Okamoto H, Horai T, et al. A randomized phase III study of single-agent amrubicin vs. carboplatin/ etoposide in elderly patients with extensive-disease small-cell lung cancer. Clin Lung Cancer. 2014;15(2):96-102.

22. Socinski MA, Smit EF, Lorigan P, et al. Phase III study of pemetrexed plus carboplatin compared with etoposide plus carboplatin in chemotherapy-naive patients with extensive-stage small-cell lung cancer. J Clin Oncol. 2009;27(28):4787-4792.

23. Artal-Cortes A, Gomez-Codina J, Gonzalez-Larriba JL, et al. Prospective randomized phase III trial of etoposide/ cisplatin versus high-dose epirubicin/cisplatin in small-cell lung cancer. Clin Lung Cancer. 2004;6(3):175-183.

24. Berghmans T, Scherpereel A, Meert AP, et al. A Phase III Randomized Study Comparing a Chemotherapy with Cisplatin and Etoposide to a Etoposide Regimen without Cisplatin for Patients with Extensive Small-Cell Lung Cancer. Front Oncol. 2017;7:217

25. Eckardt JR, von Pawel J, Papai Z, et al. Open-label, multicenter, randomized, phase III study comparing oral topotecan/cisplatin versus etoposide/cisplatin as treatment for chemotherapy-naive patients with extensive-disease small-cell lung cancer. J Clin Oncol. 2006;24(13):2044-2051.

26. Fink TH, Huber RM, Heigener DF, et al. Topotecan/cisplatin compared with cisplatin/etoposide as first-line treatment for patients with extensive disease small-cell lung cancer: final results of a randomized phase III trial. J Thorac Oncol. 2012;7(9):1432-1439.

27. Hanna N, Bunn PA, Jr., Langer C, et al. Randomized phase III trial comparing irinotecan/cisplatin with etoposide/ cisplatin in patients with previously untreated extensive-stage disease small-cell lung cancer. J Clin Oncol. 2006;24(13):2038-2043.

28. Kim DW, Kim HG, Kim JH, et al. Randomized Phase III Trial of Irinotecan Plus Cisplatin versus Etoposide Plus Cisplatin in Chemotherapy-Naive Korean Patients with Extensive-Disease Small Cell Lung Cancer. Cancer Res Treat. 2019;51(1):119-127.

29. Lara PN, Jr., Natale R, Crowley J, et al. Phase III trial of irinotecan/cisplatin compared with etoposide/cisplatin in extensive-stage small-cell lung cancer: clinical and pharmacogenomic results from SWOG S0124. J Clin Oncol. 2009;27(15):2530-2535.

30. Loehrer PJ, Sr., Ansari R, Gonin R, et al. Cisplatin plus etoposide with and without ifosfamide in extensive small-cell lung cancer: a Hoosier Oncology Group study. J Clin Oncol. 1995;13(10):2594-2599.

31. Niell HB, Herndon JE, 2nd, Miller AA, et al. Randomized phase III intergroup trial of etoposide and cisplatin with or without paclitaxel and granulocyte colony-stimulating factor in patients with extensive-stage small-cell lung cancer: Cancer and Leukemia Group B Trial 9732. J Clin Oncol. 2005;23(16):3752-3759.

32. Noda K, Nishiwaki Y, Kawahara M, et al. Irinotecan plus cisplatin compared with etoposide plus cisplatin for extensive small-cell lung cancer. N Engl J Med. 2002;346(2):85-91.

33. Oh IJ, Kim KS, Park CK, et al. Belotecan/cisplatin versus etoposide/cisplatin in previously untreated patients with extensive-stage small cell lung carcinoma: a multi-center randomized phase III trial. BMC Cancer. 2016;16:690.

34. Pujol JL, Daures JP, Riviere A, et al. Etoposide plus cisplatin with or without the combination of 4'-epidoxorubicin plus cyclophosphamide in treatment of extensive small-cell lung cancer: a French Federation of Cancer Institutes multicenter phase III randomized study. J Natl Cancer Inst. 2001;93(4):300-308.

35. Roth BJ, Johnson DH, Einhorn LH, et al. Randomized study of cyclophosphamide, doxorubicin, and vincristine versus etoposide and cisplatin versus alternation of these two regimens in extensive small-cell lung cancer: a phase III trial of the Southeastern Cancer Study Group. J Clin Oncol. 1992;10(2):282-291.

36. Rowland KM, Jr., Loprinzi CL, Shaw EG, et al. Randomized double-blind placebo-controlled trial of cisplatin and etoposide plus megestrol acetate/placebo in extensive-stage small-cell lung cancer: a North Central Cancer Treatment Group study. J Clin Oncol. 1996;14(1):135-141.

37. Shi Y, Hu Y, Hu X, Li X, Lin L, Han X. Cisplatin combined with irinotecan or etoposide for untreated extensivestage small cell lung cancer: A multicenter randomized controlled clinical trial. Thorac Cancer. 2015;6(6):785-791.

38. Sun Y, Cheng Y, Hao X, et al. Randomized phase III trial of amrubicin/cisplatin versus etoposide/cisplatin as first-line treatment for extensive small-cell lung cancer. BMC Cancer. 2016;16:265.

39. Sundstrom S, Bremnes RM, Kaasa S, et al. Cisplatin and etoposide regimen is superior to cyclophosphamide, epirubicin, and vincristine regimen in small-cell lung cancer: results from a randomized phase III trial with 5 years' follow-up. J Clin Oncol. 2002;20(24):4665-4672. 
40. Tiseo M, Boni L, Ambrosio F, et al. Italian, Multicenter, Phase III, Randomized Study of Cisplatin Plus Etoposide With or Without Bevacizumab as First-Line Treatment in Extensive-Disease Small-Cell Lung Cancer: The GOIRC-AIFA FARM6PMFJM Trial. J Clin Oncol. 2017;35(12):1281-1287.

41. Zatloukal P, Cardenal F, Szczesna A, et al. A multicenter international randomized phase III study comparing cisplatin in combination with irinotecan or etoposide in previously untreated small-cell lung cancer patients with extensive disease. Ann Oncol. 2010;21(9):1810-1816.

42. Zhang J, Qi HW, Zheng H, et al. Etoposide-cisplatin alternating with vinorelbine-cisplatin versus etoposide-cisplatin alone in patients with extensive disease combined with small cell lung cancer. Asian Pac J Cancer Prev. 2014;15(10):4159-4163.

43. de Jong WK, Groen HJ, Koolen MG, et al. Phase III study of cyclophosphamide, doxorubicin, and etoposide compared with carboplatin and paclitaxel in patients with extensive disease small-cell lung cancer. Eur J Cancer. 2007;43(16):2345-2350.

44. Postmus PE, Scagliotti G, Groen HJ, et al. Standard versus alternating non-cross-resistant chemotherapy in extensive small cell lung cancer: an EORTC Phase III trial. Eur J Cancer. 1996;32A(9):1498-1503. 


\section{APPENDICES CHAPTER 7}

APPENDIX 1 Literature search

APPENDIX 2 Flow charts and reference tables clinical trial outcomes

Table 1. Clinical trial reference outcomes

Figure 1. Flowchart carboplatin-etoposide

Figure 2. Forest plot carboplatin -etoposide

Table 2. Clinical trial and real-world characteristics regimen carboplatin -etoposide Figure 3. Flowchart cisplatin -etoposide

Figure 4. Forest plot cisplatin -etoposide

Table 3. Clinical trial and real-world characteristics regimen cisplatin -etoposide

Figure 5. Flowchart cyclophosfamide-doxorubicin-etoposide (CDE)

Figure 6. Forest plot cyclophosfamide-doxorubicin-etoposide (CDE)

Table 4. Clinical trial and real-world characteristics regimen cyclophosfamidedoxorubicin-etoposide (CDE)

Figure 7. Forest plot with overview of clinical trials for reference outcome first-line chemotherapy 


\section{APPENDIX 1 LITERATURE SEARCH}

\section{PubMed; search on September 12, 2018 \\ Carboplatin-etoposide}

(carboplatin*[tiab] OR "Carboplatin"[Mesh]) AND ("Etoposide"[Mesh] OR etoposide[tiab]) AND ("Small Cell Lung Carcinoma"[Mesh] OR small-cell-lung-c*[tiab] OR sclc[tiab]) AND (extensive[tiab] OR advanced[tiab] OR stage IV[tiab] OR stage 4[tiab]) AND ("mortality" [Subheading] OR "Mortality"[Mesh] OR "Survival"[Mesh] OR "Survival Analysis"[Mesh] OR mortality[tiab] OR death*[tiab] OR survival[tiab]) NOT ("animals"[mesh] NOT "humans" [mesh]) AND ("Clinical Trial, Phase III"[pt] OR phase IIIti] OR phase 3[ti] OR (“"controlled clinical trial"[pt] OR controlled clinical trial[ti] OR randomized controlled trial[ti] OR randomized clinical trial[ti] OR randomised controlled trial[ti] OR randomised clinical trial[ti] OR rct[ti]) NOT ("Clinical Trial, Phase II"[pt] OR phase II[ti] OR phase 2[ti]))) AND english[la] NOT ("Carcinoma, Non-Small-Cell Lung"[Mesh] NOT "Small Cell Lung Carcinoma"[Mesh] NOT "Carcinoma, Small Cell"[Mesh])

\section{Cisplatin-etoposide}

(cisplatin*[tiab] OR "Cisplatin"[Mesh]) AND ("Etoposide"[Mesh] OR etoposide[tiab]) AND ("Small Cell Lung Carcinoma"[Mesh] OR small-cell-lung-c*[tiab] OR sclc[tiab]) AND (extensive[tiab] OR advanced[tiab] OR stage IV[tiab] OR stage 4[tiab]) AND ("mortality" [Subheading] OR "Mortality"[Mesh] OR "Survival"[Mesh] OR "Survival Analysis"[Mesh] OR mortality[tiab] OR death*[tiab] OR survival[tiab]) NOT ("animals"[mesh] NOT "humans" [mesh]) AND ("Clinical Trial, Phase III"[pt] OR phase IIIti] OR phase 3[ti] OR (("controlled clinical trial"[pt] OR controlled clinical trial[ti] OR randomized controlled trial[ti] OR randomized clinical trial[ti] OR randomised controlled trial[ti] OR randomised clinical trial[ti] OR rct[ti]) NOT ("Clinical Trial, Phase II"[pt] OR phase II[ti] OR phase 2[ti]))) AND english[la] NOT ("Carcinoma, Non-Small-Cell Lung"[Mesh] NOT "Small Cell Lung Carcinoma"[Mesh] NOT "Carcinoma, Small Cell"[Mesh])

\section{Cyclophosfamide-doxorubicin-etoposide}

(cyclophosphamide[tiab] OR "Cyclophosphamide"[Mesh]) AND ("Etoposide"[Mesh] OR etoposide[tiab]) AND (doxorubicin[tiab] OR "Doxorubicin"[Mesh] OR adriamycin[tiab]) AND ("Small Cell Lung Carcinoma"[Mesh] OR small-cell-lung-c*[tiab] OR sclc[tiab]) AND (extensive[tiab] OR advanced[tiab] OR stage IV[tiab] OR stage 4[tiab]) AND ("mortality" [Subheading] OR "Mortality"[Mesh] OR "Survival"[Mesh] OR "Survival Analysis"[Mesh] OR mortality[tiab] OR death*[tiab] OR survival[tiab]) NOT ("animals"[mesh] NOT "humans" [mesh]) AND ("Clinical Trial, Phase III"[pt] OR phase IIIti] OR phase 3[ti] OR (“"controlled clinical trial"[pt] OR controlled clinical trial[ti] OR randomized controlled trial[ti] OR randomized clinical trial[ti] OR randomised controlled trial[ti] OR randomised clinical trial[ti] OR rct[ti]) NOT ("Clinical Trial, Phase II"[pt] OR phase II[ti] OR phase 2[ti]))) AND 
english[la] NOT ("Carcinoma, Non-Small-Cell Lung"[Mesh] NOT "Small Cell Lung Carcinoma"[Mesh] NOT "Carcinoma, Small Cell"[Mesh])

\section{EMBASE; search on September 12, 2018 Carboplatin-etoposide}

(('carboplatin'/exp OR carboplatin*:ab,ti) AND ('etoposide'/exp OR etoposide:ab,ti)) AND ('small cell lung cancer'/exp OR 'small cell lung cancer':ab,ti OR SCLC :ab,ti) AND ('extensive stage small cell lung cancer'/exp OR 'extensive disease small cell lung cancer'/exp OR 'stage IV':ab,ti OR 'stage 4':ab,ti OR advanced:ab,ti) AND ('mortality'/exp OR 'survival'/exp OR mortality:ab,ti OR death*:ab,ti OR survival:ab,ti) AND ('phase 3 clinical trial'/exp OR 'phase III':ti OR 'phase 3':ti OR 'controlled clinical trial'/exp OR 'controlled clinical trial':ti OR 'randomized controlled trial':ti OR 'randomized clinical trial':ti OR 'randomised controlled trial':ti OR 'randomised clinical trial':ti OR rct:ti NOT ('phase 2 clinical trial'/exp OR 'phase ll':ti OR 'phase 2':ti)) AND [english]/lim NOT 'conference abstract'/it NOT ('non small cell lung cancer'/exp NOT 'small cell carcinoma'/exp)

\section{Cisplatin-etoposide}

(('cisplatin'/exp OR cisplatin*:ab,ti) AND ('etoposide'/exp OR etoposide:ab,ti)) AND ('small cell lung cancer'/exp OR 'small cell lung cancer':ab,ti OR SCLC :ab,ti) AND ('extensive stage small cell lung cancer'/exp OR 'extensive disease small cell lung cancer'/exp OR 'stage IV':ab,ti OR 'stage 4':ab,ti OR advanced:ab,ti) AND ('mortality'/exp OR 'survival'/exp OR mortality:ab,ti OR death*:ab,ti OR survival:ab,ti) AND ('phase 3 clinical trial'/exp OR 'phase IIl':ti OR 'phase 3':ti OR 'controlled clinical trial'/exp OR 'controlled clinical trial':ti OR 'randomized controlled trial':ti OR 'randomized clinical trial':ti OR 'randomised controlled trial':ti OR 'randomised clinical trial':ti OR rct:ti NOT ('phase 2 clinical trial'/exp OR 'phase ll':ti OR 'phase 2':ti)) AND [english]/lim NOT 'conference abstract'/it NOT ('non small cell lung cancer'/exp NOT 'small cell carcinoma'/exp)

\section{Cyclophosfamide-doxorubicin-etoposide}

(('cyclophosphamide'/exp OR cyclophosphamide:ab,ti) AND ('etoposide'/exp OR etoposide:ab,ti) AND ('doxorubicin'/exp OR doxorubicin:ab,ti OR adriamycin:ab,ti)) AND ('small cell lung cancer'/exp OR 'small cell lung cancer':ab,ti OR SCLC*:ab,ti) AND ('extensive stage small cell lung cancer'/exp OR 'extensive disease small cell lung cancer'/exp OR 'stage IV':ab,ti OR 'stage 4':ab,ti OR advanced:ab,ti) AND ('mortality'/exp OR 'survival'/exp OR mortality:ab,ti OR death*:ab,ti OR survival:ab,ti) AND ('phase 3 clinical trial'/exp OR 'phase IIl'ti OR 'phase 3':ti OR 'controlled clinical trial'/exp OR 'controlled clinical trial':ti OR 'randomized controlled trial':ti OR 'randomized clinical trial':ti OR 'randomised controlled trial':ti OR 'randomised clinical trial':ti OR rct:ti NOT ('phase 2 clinical trial'/exp OR 'phase Il':ti OR 'phase 2':ti)) AND [english]/lim NOT 'conference abstract'/it NOT ('non small cell lung cancer'/exp NOT 'small cell carcinoma'/exp) 


\section{Cochrane; search on September 12, 2018}

\section{Carboplatin-etoposide}

(carboplatin* AND etoposide):ti,ab,kw AND ("phase 3" OR "phase III"):ti,ab,kw AND ("small cell lung cancer" OR SCLC OR "small cell lung carcinoma"):ti,ab,kw AND (extensive OR advanced OR "stage 4" OR "stage IV"):ti,ab,kw AND (mortality OR death* OR survival):ti,ab,kw NOT (Carcinoma, Non-Small-Cell Lung NOT Small Cell Lung Carcinoma):ti,ab,kw

\section{Cisplatin-etoposide}

(cisplatin* AND etoposide):ti,ab,kw AND ("phase 3" OR "phase III"):ti,ab,kw AND ("small cell lung cancer" OR SCLC OR "small cell lung carcinoma"):ti,ab,kw AND (extensive OR advanced OR "stage 4" OR "stage IV"):ti,ab,kw AND (mortality OR death* OR survival):ti,ab,kw NOT (Carcinoma, Non-Small-Cell Lung NOT Small Cell Lung Carcinoma):ti,ab,kw

\section{Cyclophosfamide-doxorubicin-etoposide}

("cyclophosphamide" AND doxorubicin OR adriamycin AND etoposide):ti,ab,kw AND ("phase 3" OR "phase III"):ti,ab,kw AND ("small cell lung cancer" OR SCLC OR "small cell lung carcinoma"):ti,ab,kw AND (extensive OR advanced OR "stage 4" OR "stage IV"):ti,ab,kw AND (mortality OR death* OR survival):ti,ab,kw 


\section{APPENDIX 2 FLOW CHARTS AND REFERENCE TABLES CLINICAL TRIAL OUTCOMES}

Table 1. Clinical trial reference outcomes

\begin{tabular}{lc}
\hline Chemotherapy regimen & $\begin{array}{c}\text { Median OS clinical trials } \\
\text { (months) }\end{array}$ \\
\hline Carboplatin-etoposide & 9.48 \\
Cisplatin-etoposide & 9.43 \\
Cyclophosfamide-doxorubicin-etoposide & 7.29 \\
Overall & $\mathbf{9 . 3 3}$ \\
\hline
\end{tabular}




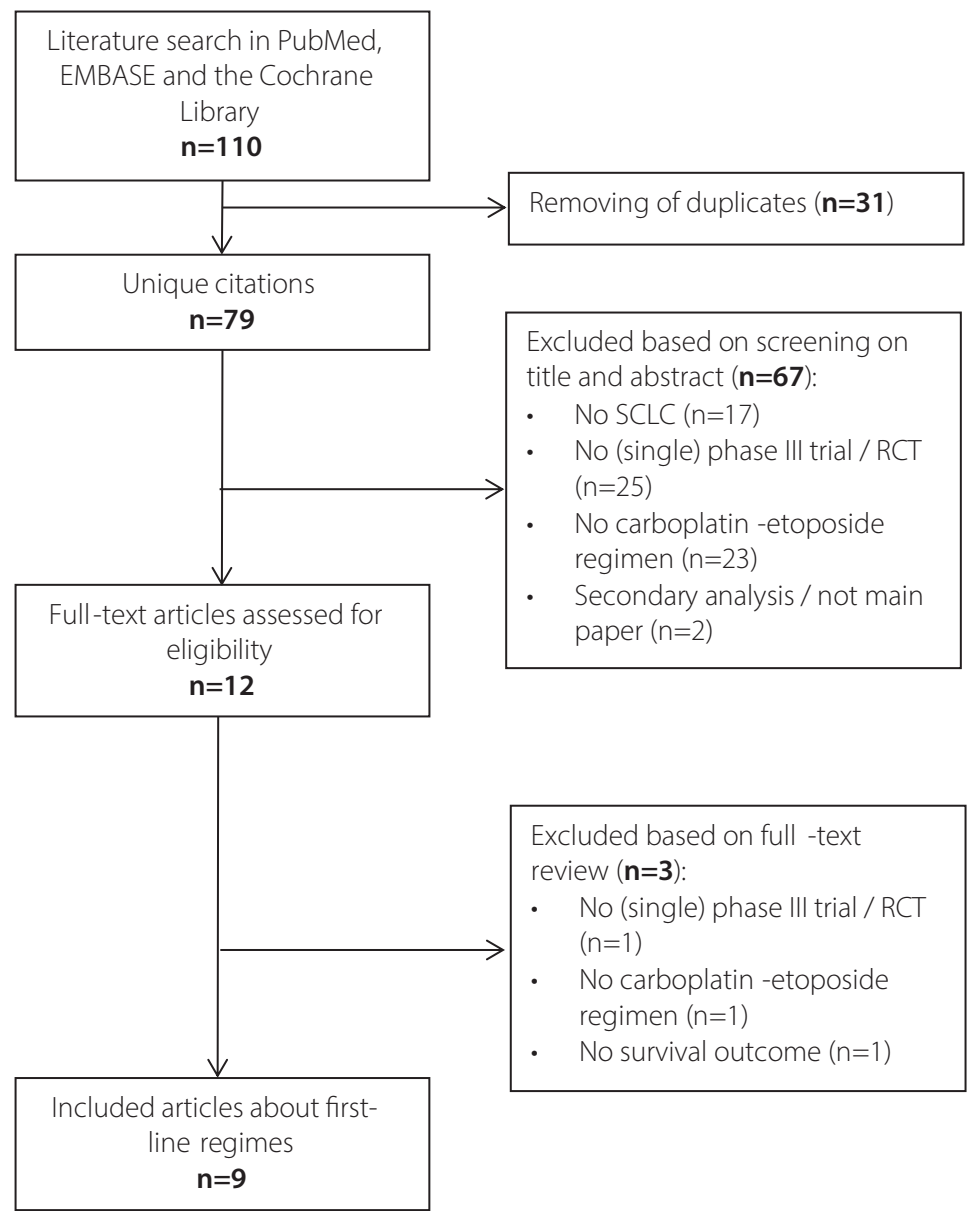

Figure 1. Flowchart carboplatin-etoposide 


\begin{tabular}{|c|c|c|c|c|c|c|c|c|}
\hline Study or Subgroup & Mean survival time & SE & Weight & $\begin{array}{l}\text { Mean survival time } \\
\text { IV, Random, } 95 \% \mathrm{Cl}\end{array}$ & & $\begin{array}{l}\text { Mea } \\
\text { IN, R }\end{array}$ & $\begin{array}{l}\text { urvival time } \\
\text { lom, } 95 \% \mathrm{Cl}\end{array}$ & \\
\hline Heigener 2009 & 11.2 & 1.5561 & $5.1 \%$ & $11.20[8.15,14.25]$ & & & & \\
\hline Hermes 2008 & 7.1 & 0.4772 & $13.8 \%$ & $7.10[6.16,8.04]$ & & & & - \\
\hline Jalal 2017 & 10.37 & 1.199 & $7.1 \%$ & $10.37[8.02,12.72]$ & & & & \\
\hline Lee 2009 & 9.1 & 0.2948 & $15.5 \%$ & $9.10[8.52,9.68]$ & & & & $=$ \\
\hline Okamoto 2007 & 10.6 & 0.6632 & $11.8 \%$ & $10.60[9.30,11.90]$ & & & & $\rightarrow$ \\
\hline Schmittel 2011 & 9 & 0.7143 & $11.3 \%$ & $9.00[7.60,10.40]$ & & & & $\rightarrow$ \\
\hline Seckl 2017 & 8.8 & 0.2831 & $15.6 \%$ & $8.80[8.25,9.35]$ & & & & - \\
\hline Sekine 2014 & 11.3 & 1.352 & $6.1 \%$ & $11.30[8.65,13.95]$ & & & & $\longrightarrow$ \\
\hline Socinski 2009 & 10.6 & 0.4847 & $13.7 \%$ & $10.60[9.65,11.55]$ & & & & $\rightarrow$ \\
\hline Total $(95 \% \mathrm{Cl})$ & & & $100.0 \%$ & $9.48[8.66,10.30]$ & & & & 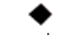 \\
\hline \multicolumn{5}{|c|}{$\begin{array}{l}\text { Heterogeneity: } \mathrm{Tau}^{2}=1.04 ; \mathrm{Chi}^{2}=38.99, \mathrm{df}=8(\mathrm{P}<0.00001) ; \mathrm{I}^{2}=79 \% \\
\text { Test for overall effect: } Z=22.66(\mathrm{P}<0.00001)\end{array}$} & -10 & -5 & $\begin{array}{ll} & 5 \\
0 & 5 \\
\text { Overall sur }\end{array}$ & $\begin{array}{c}10 \\
10 \\
\text { uvival }(\mathrm{m}\end{array}$ \\
\hline
\end{tabular}

Figure 2. Forest plot carboplatin-etoposide

Table 2. Clinical trial and real-world characteristics regimen carboplatin-etoposide

\begin{tabular}{lcc}
\hline Characteristic & Clinical trials & Real-world \\
\hline Total number of patients & 1351 & 335 \\
Overall survival (months) & 9.48 & 7.23 \\
Median age (years) & 63 & 68 \\
Male (\%) & 68 & 61 \\
Stage IV (\%) & 89 & 100 \\
ECOG performance score 0-1 (\%) & 80 & 74 \\
\hline
\end{tabular}




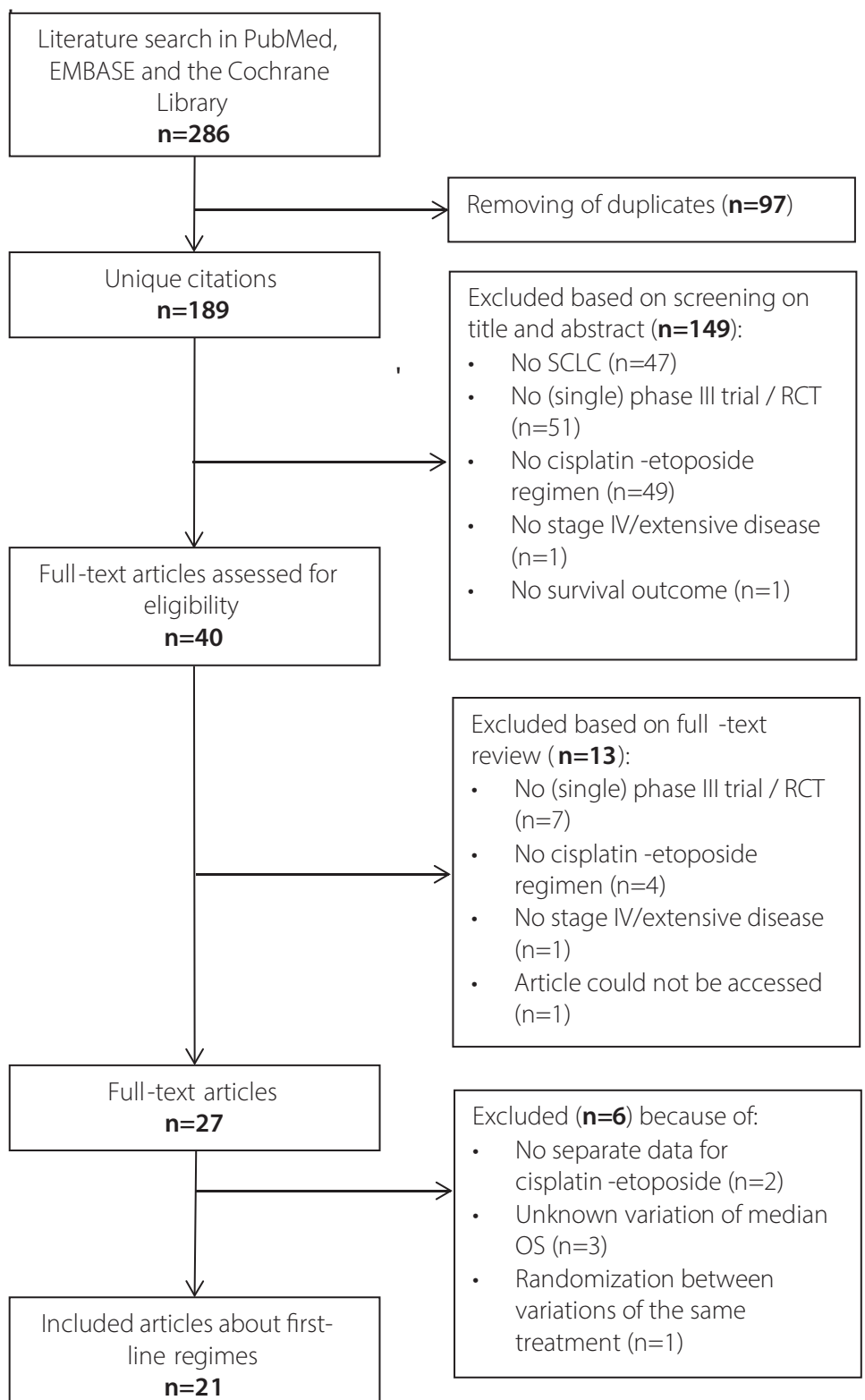

Figure 3. Flowchart cisplatin-etoposide 


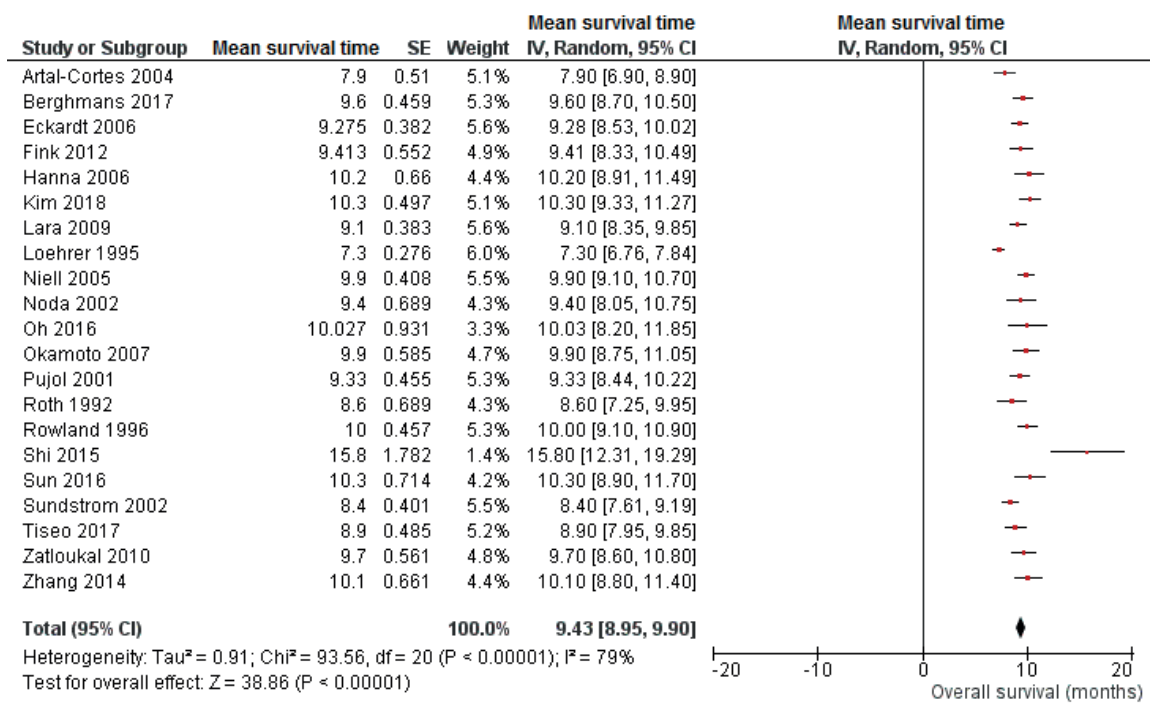

Figure 4. Forest plot cisplatin-etoposide

Table 3. Clinical trial and real-world characteristics regimen cisplatin-etoposide

\begin{tabular}{lcc}
\hline Characteristic & Clinical trials & Real-world \\
\hline Total number of patients & 3285 & 209 \\
Overall survival (months) & 9.43 & 8.18 \\
Median age (years) & $62^{\mathrm{a}}$ & 64 \\
Male (\%) & 76 & 45 \\
Stage IV / extensive disease (\%) & 97 & 100 \\
ECOG performance score 0-1 (\%) & $84^{\mathrm{b}}$ & 76 \\
\hline & & \\
a Note: missing in 1 study reference & & \\
b Note: missing in 4 study references & &
\end{tabular}




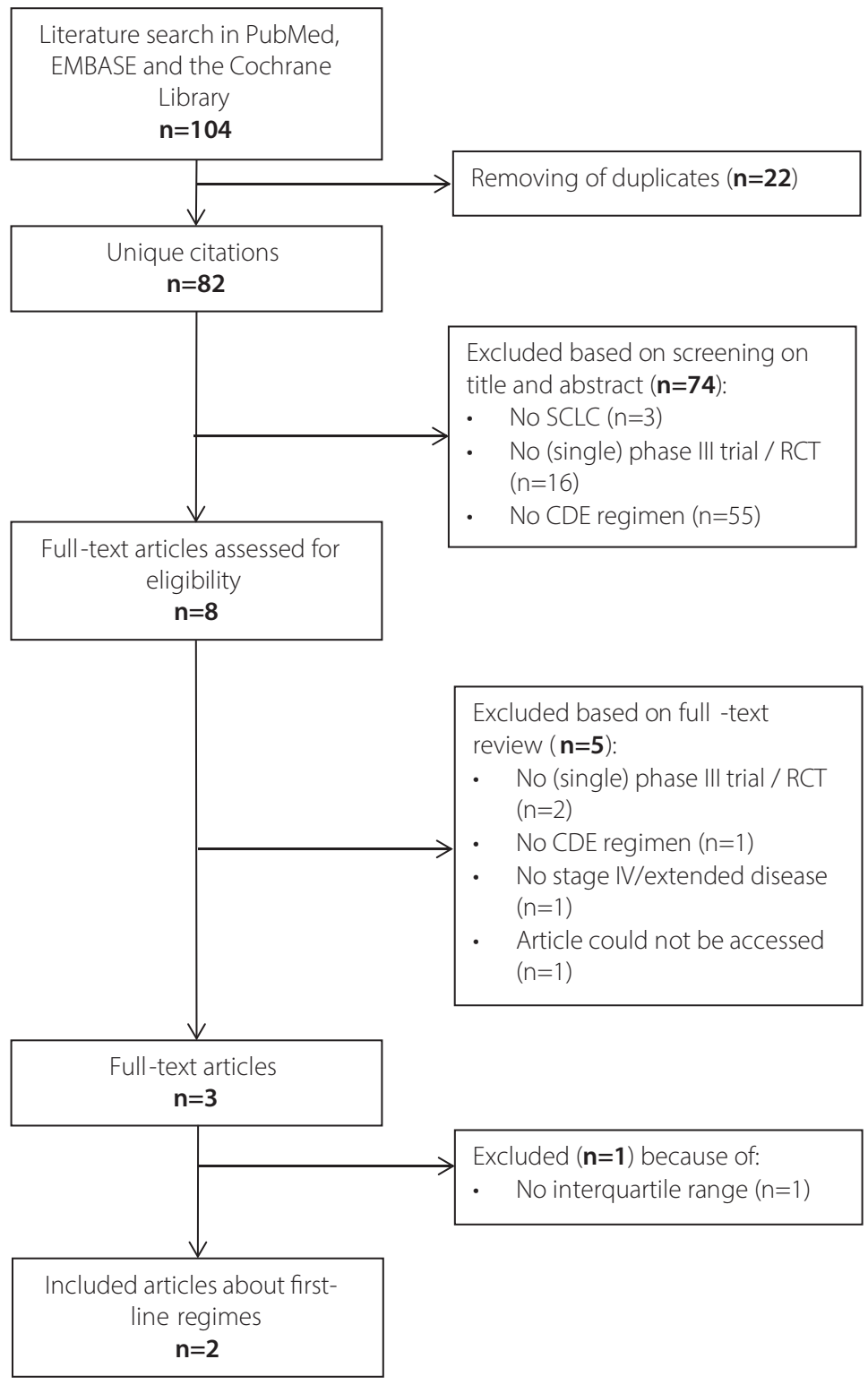

Figure 5. Flowchart cyclophosfamide-doxorubicin-etoposide (CDE) 


\begin{tabular}{|c|c|c|c|c|c|c|c|}
\hline Study or Subgroup & Mean survival time & SE & Weight & $\begin{array}{l}\text { Mean survival time } \\
\text { IV, Fixed, } 95 \% \mathrm{Cl}\end{array}$ & & $\begin{array}{l}\text { Mean survival time } \\
\text { IV, Fixed, } 95 \% \mathrm{Cl}\end{array}$ & \\
\hline de Jong 2007 & 6.8 & 0.9184 & $39.1 \%$ & $6.80[5.00,8.60]$ & & & $\longrightarrow$ \\
\hline Postmus 1996 & 7.6 & 0.7352 & $60.9 \%$ & $7.60[6.16,9.04]$ & & & \\
\hline Total (95\% Cl) & & & $100.0 \%$ & $7.29[6.16,8.41]$ & & & \\
\hline \multicolumn{4}{|c|}{$\begin{array}{l}\text { Heterogeneity: } C \mathrm{Ch}^{2}=0.46, \mathrm{df}=1(\mathrm{P}=0.50) ; \mathrm{I}^{2}=0 \% \\
\text { Test for overall effect: } Z=12.70(P<0.00001)\end{array}$} & -10 & -5 & Overall sur & $\begin{array}{ll} \\
5 & 10 \\
\text { rvival (months) }\end{array}$ \\
\hline
\end{tabular}

Figure 6. Forest plot cyclophosfamide-doxorubicin-etoposide (CDE)

Table 4. Randomized clinical trial characteristics

\begin{tabular}{lcc}
\hline Characteristic & Clinical trials & Real-world \\
\hline Total number of patients & 175 & 15 \\
Pooled overall survival (months) & 7.29 & 5.62 \\
Median age (years) & 61 & 68 \\
Male (\%) & 69 & 87 \\
Stage IV (\%) & 100 & 100 \\
ECOG performance score 0-1 (\%) & 74 & 73 \\
\hline
\end{tabular}




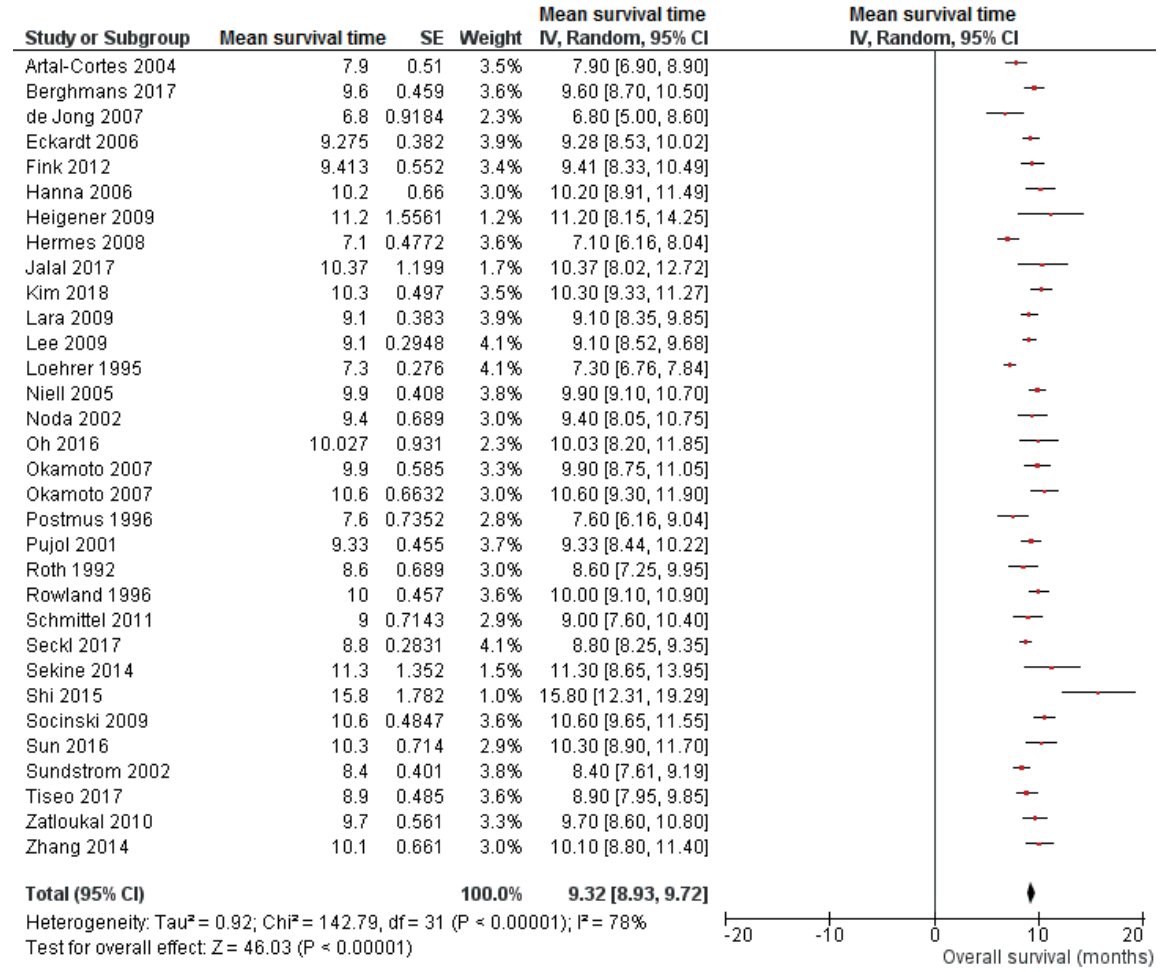

Figure 7. Forest plot with overview of clinical trials for reference outcome first-line chemotherapy 


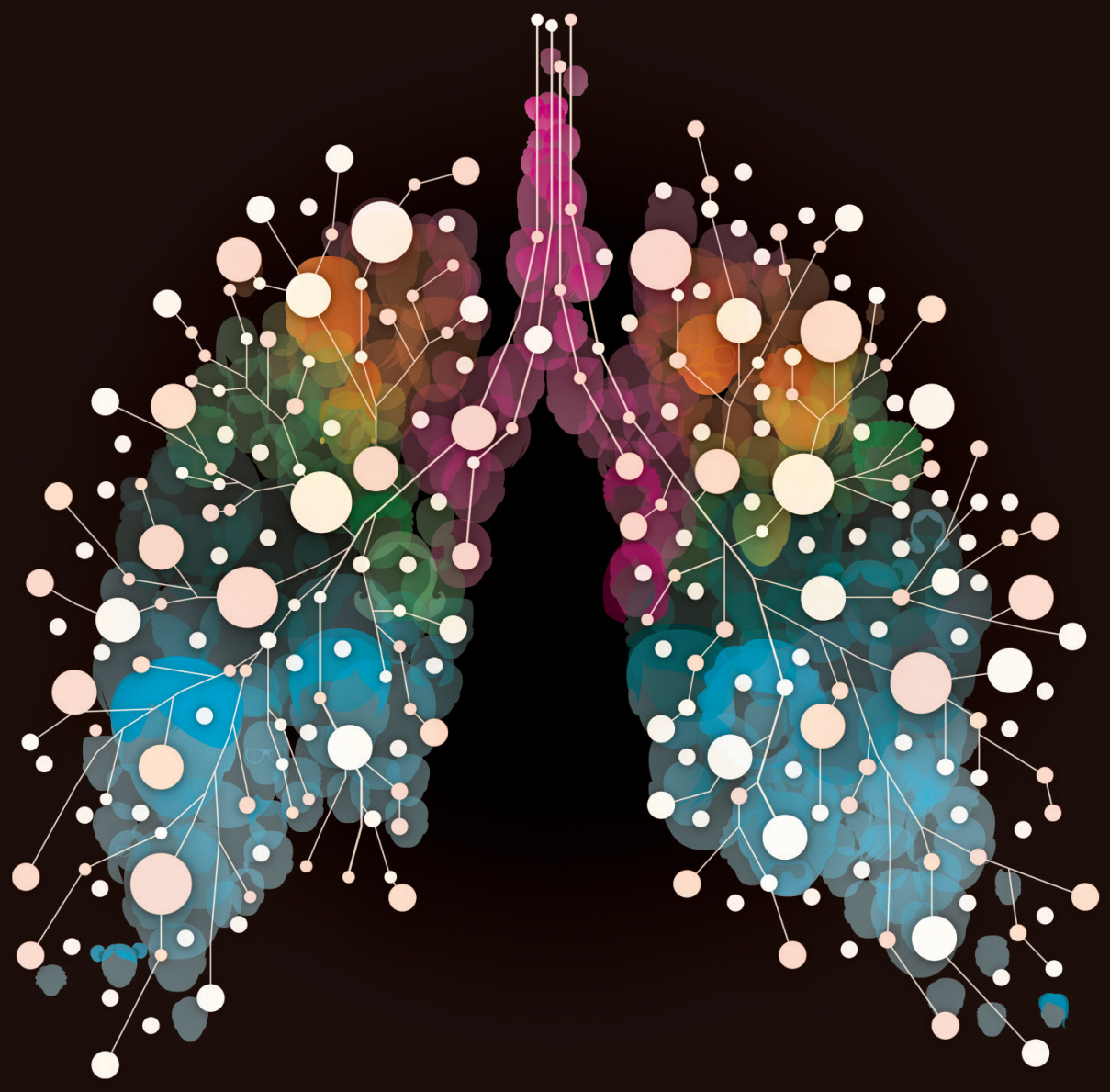


PART III

Communication of real-world outcomes to patients 



\title{
Chapter 8
}

Development and evaluation of a real-world outcomes based tool to support informed clinical decision-making in the palliative treatment of patients with metastatic non-small cell lung cancer

\author{
Christine M. Cramer-van der Welle, Elisabeth A. Kastelijn, Bram C. Plouvier, \\ Cornelia F. van Uden-Kraan, Franz M.N.H. Schramel, Harry J.M. Groen, \\ Ewoudt M.W. van de Garde
}

Submitted 


\section{ABSTRACT}

Purpose: To develop and evaluate a tool for patients with stage IV NSCLC and their thoracic oncologists (TOs) that provides insight in real-world effectiveness of systemic treatments to support informed clinical decision-making in the palliative setting.

Methods: A participatory design approach was used to acquire insights from patients and TOs into preferences regarding the content and design of the web-based tool. Implementation was investigated by means of an adoption and usage rate. The appreciation of the tool was evaluated through a telephone survey with patients and a questionnaire for TOs.

Results: From data of 2989 patients with stage IV NSCLC diagnosed in one of the Santeon hospitals, an interface was developed to show treatments plus both real-world outcomes and clinical trial results after selecting patient characteristics ('patients like me'). This prototype of the tool was finalised after discussion in a focus group with 4 TOs and semi-structured interviews with 6 patients. The tool was implemented and used by TOs in three of six Santeon hospitals (50\% adoption rate). The tool was effectively used in 48 patients (29\% usage rate) of which 17 participated in the telephone survey. Ten TOs responded to the questionnaire. The responses varied from positive reactions on the clear overview on treatment outcomes to statements that the tool rarely changed treatment decisions. Overall, the majority of patients and TOs scored the tool as of added value (71\% and 83\%, respectively).

Conclusion: Our real-world data tool in metastatic lung cancer was appreciated in clinical practice by both patients and TOs. However, the efficacy of the implementation can be improved. 


\section{INTRODUCTION}

Most oncologists use survival and toxicity data from clinical trials to counsel patients, inform decision-making, and obtain consent for treatment. The same applies to the creation of treatment guidelines. It is known, however, that patients treated in everyday practice tend to be older and more frail, have poorer performance status, and have more comorbidities and less social support than those selected to participate in clinical trials. ${ }^{2}$ This makes the translation of outcomes data from clinical trials to regular practice complex. Previously, we found that survival of patients with metastatic NSCLC treated with systemic therapy in real-world practice is nearly one quarter shorter than for patients included in clinical trials, and stopping treatment for toxicity is more frequent in real-world. ${ }^{3}$ Therefore, in addition to clinical trial efficacy data, data about clinical effectiveness of systemic treatment options in metastatic NSCLC from real-world settings can be useful to provide to patients an overview of what can be expected from different treatment options. Such information could help patients to consider their options from a personal view (e.g., how important the possible benefits and harms are to them) and could help them to be involved in making a decision together with their health practitioner. ${ }^{4}$ Additionally, real-world data can assist thoracic oncologists (TOs) in formulating treatment options with enough gain in overall survival to be worthwhile for patients, as research has shown that oncologists tend to overestimate life expectancy of patients, and that they could benefit from using tools providing individual survival estimates. ${ }^{5}$

The potential added value of providing real-world outcomes data through a tool has not been studied before in patients with metastatic NSCLC. A review by Révész et al. on available decision support systems (DSS) for incurable patients with metastatic NSCLC reported that all DSS were based on statistical models that make use of probabilities (e.g., prediction models or nomograms) and lacked recent clinical data. ${ }^{6}$ Furthermore, there is no DSS that gives an overview of all treatments relevant to consider in incurable NSCLC patients, or that offers information about intensive treatment versus best supportive care. Additionally, Révész et al. examined the needs and preferences of TOs with regard to future DSS in the treatment of metastatic NSCLC and reported that $77 \%$ would welcome new DSS for their own decision-making and/or for shared decision-making. ${ }^{5}$

The aim of the present study was to develop a tool for patients with stage IV NSCLC and their TOs that provides insight in the real-world effectiveness of systemic treatments ('patients like me' concept) to support well-informed treatment decisions in the palliative setting, and to evaluate the appreciation of the tool in routine clinical practice. 


\section{METHODS}

Figure 1 shows the steps conducted in the development and evaluation phases of the tool. A participatory design approach was used to acquire insights from patients and TOs into preferences regarding the content and design of the web-based tool. ${ }^{7}$

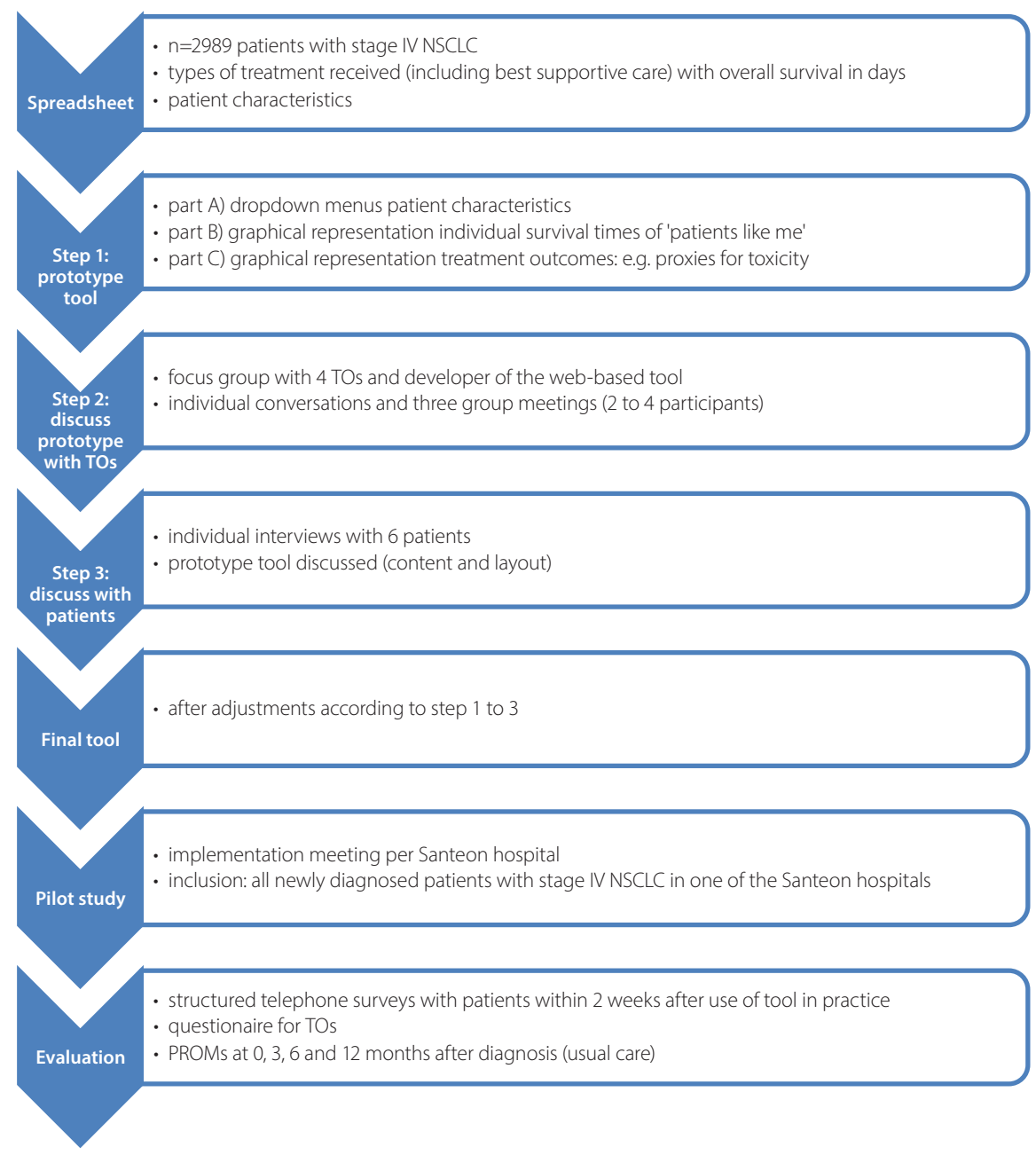

Figure 1. Flow chart of the steps conducted in development, pilot study, and evaluation phase of the tool. NSCLC: non-small cell lung cancer; TOs: thoracic oncologists; PROMs: Patient Reported Outcome Measures 


\section{Development of the tool}

From our previous research ( $\mathrm{n}=2989$ patients with stage IV NSCLC) ${ }^{3}$, data on patient characteristics, types of treatment received (including best supportive care), survival, and toxicity-related outcomes were extracted per patient. These data were collated in a spreadsheet that formed the base for the present project.

\section{Step 1 Prototype based on available data}

Based on the first-line treatment regimens in the spreadsheet, a prototype of the tool was designed which included three parts: A) dropdown menus for all available patient characteristics (e.g. age, gender, ECOG performance status (PS), co-morbidities), to be entered by the TO; B) graphical representation of individual survival times of prior real-world patients resembling the patient characteristics within a selection; and C) graphical representation of treatment outcomes: e.g. proxies for toxicity such as percentage of the resembling patients with dose reduction, and most common side effects of the systemic treatments shown.

\section{Step 2 Focus group and meetings with TOs}

To ensure a good understanding of the complex data to be presented in the tool, the exact lay-out of the interface and how the data should be presented was determined in close collaboration with TOs from three Santeon hospitals in the Netherlands (St. Antonius Hospital Nieuwegein/Utrecht, OLVG Hospital Amsterdam, Canisius-Wilhelmina Hospital Nijmegen). The prototype of the tool (step 1) was discussed in a focus group with 4 TOs, using a question route moving from general to more specific issues, focusing on their perceptions of applying the tool (positive features, changes needed, relevance, and timing of use).

Because of novel treatment options emerging during the development period of the tool (e.g. immunotherapy), individual conversations and three group meetings (with 2 to 4 participants) were organized to discuss the feasibility for processing these novel treatments into the tool.

\section{Step 3 Interviews with patients}

Individual semi-structured interviews with 6 patients who were recently diagnosed with stage IV NSCLC in the St. Antonius Hospital were organized to discuss and further develop the content and layout of the tool as designed in step 1 and 2. Patients were asked about their recent process of acquiring information about their diagnosis and treatment options and the idea of obtaining information through a web-based tool. The prototype of the tool was shown and discussed, including questions about interpretation of the information as shown in the tool, lacking information, suggestions for usability, timing of use, and the added value of such a tool in their decision-making process. 
The development of the tool was finalized by adjusting the three parts of the tool as mentioned in step 1, as a result of step 2 and 3.

\section{Implementation for pilot study}

The final tool was implemented in clinical practice after an instruction to all possible users in the participating hospitals (e.g. TOs, oncology nurses, pulmonologists, lung or oncology doctors in training) about the content of the tool and integration in the outpatient department. These implementation meetings were provided in a tailored manner, with a certain degree of local adaptation allowed for each hospital, with respect to the course of events as usual in their clinical practice.

The tool was offered to all newly diagnosed patients with stage IV NSCLC in the participating Santeon hospitals (step-wised introduction) starting August 1, 2019. Eligible patients were identified at the multidisciplinary tumour board (MTB) meetings of the lung cancer oncology team. Non-eligible were patients with no fluency in speaking and reading Dutch, and/or only best supportive care as possible treatment option (no treatment decision to be made). The TO (or other healthcare professional) revealing the cancer diagnosis to the patient provided the tool during the first outpatient visit of the patient after the MTB meeting, and explained the personalized information as given in the tool. If desired, the tool could also be used in a potential follow-up visit with the oncology nurse.

\section{Evaluation and measures}

The primary evaluation parameter was the added value of the tool for both patients and TOs, measured by the percentage of patients and TOs who reported availability of tooling as useful and appreciated. The added value of the tool was pre-defined as satisfying when reported by $\geq 50 \%$ of the patients or $\geq 50 \%$ of the TOs. The tool was evaluated through a structured telephone survey with patients (Appendix 1) within two weeks after using the tool in clinical practice, and a study specific questionnaire for TOs at the end of the pilot period. Information about age, gender and ECOG PS of the patient was anonymously collected from the values entered in the tool.

Secondary parameters were: 1) the adoption rate (intention to use the tool) and usage rate (actual use) ${ }^{8}$ of the tool in six Santeon hospitals, and 2) decisional roles of patients and TOs when using the tool in clinical practice, as reported with the Control Preferences Scale $\left(\mathrm{CPS}_{\text {post }}{ }^{9}\right.$ and $\mathrm{CPS}^{10}$, respectively). The adoption rate was calculated as percentage of hospitals in which TOs agreed to participate in the pilot study, and the usage rate as the percentage of patients for which the tool was actually used, based on logging data of the application. The tool was pre-defined as feasible for patients and TOs in case of an adoption rate and usage rate of over 50\%, based on those rates reported in previous studies on web-based applications.8,11 From the CPS and CPS $_{\text {post }}$ the percentage of patients and TOs with an active, collaborative or passive decisional role were calculated. 
The evaluation study was approved by a medical research ethics committee (CMO region Arnhem-Nijmegen registration number 2018-4337). All participants provided their written consent before participation in the telephone survey.

\section{Statistical analyses}

Descriptive statistics were used. To present an overview of baseline characteristics for all patients in the pilot study, frequencies (proportions) were calculated for categorical variables, and the median (with range) was provided for non-normal distributed continuous data (age). Primary and secondary parameters were presented as percentages.

\section{RESULTS}

\section{Development of the tool}

The focus group and meetings with TOs, and the semi-structured interviews with patients yielded several adjustments to the prototype of the tool. Besides a reduced number of dropdown menus with patient characteristics in part A of the tool (restricted to age, gender, and ECOG PS), dropdown menus about clinical information (type of histology, PD-L1 expression, and EGFR mutation status) were added for adequate selection of possible treatments to be shown. Secondly, adjustments were made to the graphical representation of survival outcomes in part B, to be able to display outcomes of novel treatment options emerging during the development period of the tool. Screenshots of part B and C are given in Figure 2 and Figure 3, respectively (the original Dutch interface is provided in Appendix 2). Finally, a print option for patients was added where all three parts are printed together to take home, with the possibility to discuss the information with other caregivers and family. Appendix 3 describes the development of part B and C of the tool in greater detail.

\section{Implementation for pilot study}

The meetings revealed that information which was normally provided to the patient by the TO and/or the oncology nurse about the different treatment options (e.g. information about the method of administration for the different drugs, and the number of times the patient would need to come to hospital), should be given the same way as without using the tool. Accordingly, the tool was used as source of information alongside the standard procedures and provision of information during a consultation. 


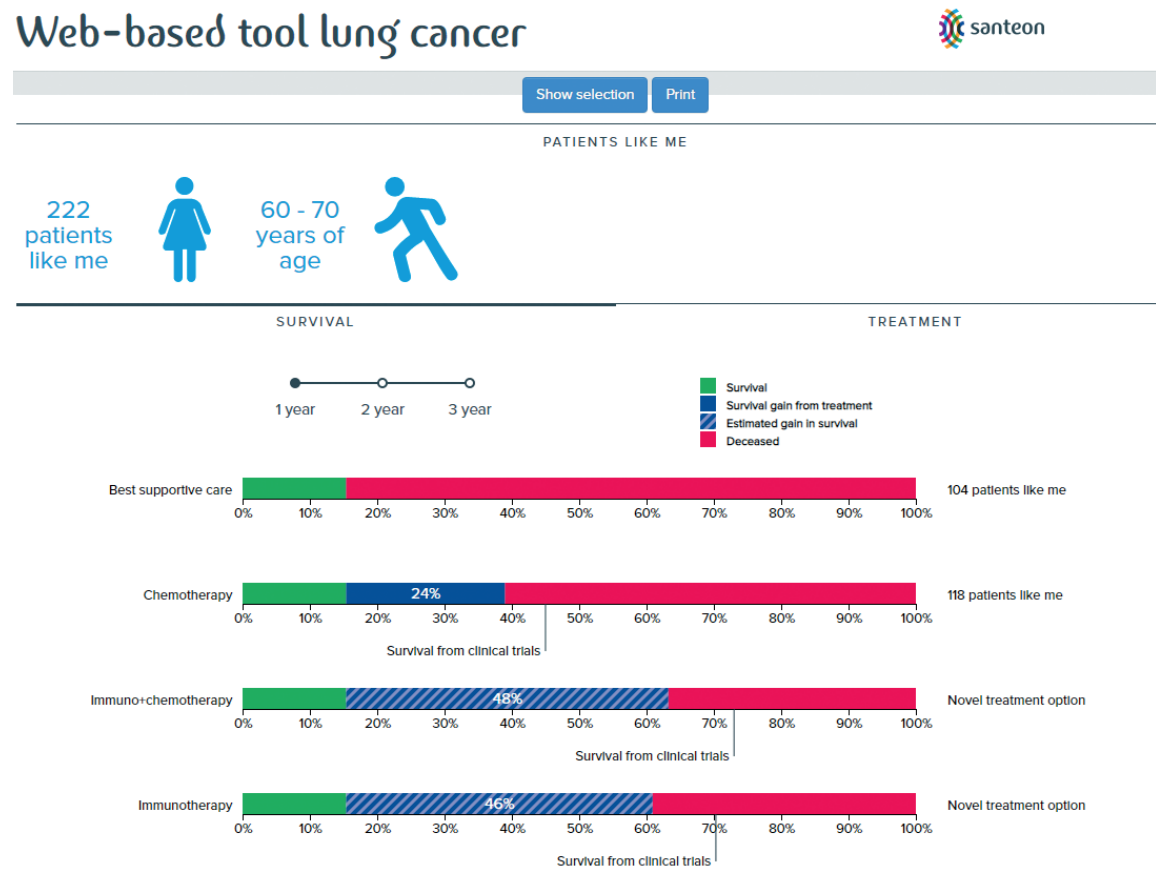

Figure 2. Screen shot of web-based tool for stage IV NSCLC patients - part B

The tool was implemented and used by TOs in three of six Santeon hospitals (50\% adoption rate). The total number of potential patients for which the tool could have been used was 166, based on the number of patients in multidisciplinary tumour board meetings in the respective pilot study periods per hospital. Eventually, the tool was used in 48 of these patients (29\% usage rate); of whom 21 were reached for the telephone survey and 17 patients actually answered the questions (Figure 4). The majority of the latter patients were male (71\%), the median age was 66, and $88 \%$ had an ECOG PS of 0-1 (Table 1).

\section{Added value of the tool (usefulness and appreciation) in pilot study} Added value of the tool according to patients

The results of the telephone survey showed that for most of the patients the information in the web-based tool was clear (94\%) and almost two third (65\%) reported that this information was important for them. Patients were positive about the overview of the different outcomes for various treatment options, for example: "It was impressive seeing the differences of the treatment options for which I was eligible". Furthermore, $71 \%$ of the patients 


\section{Web-based tool lung cancer}

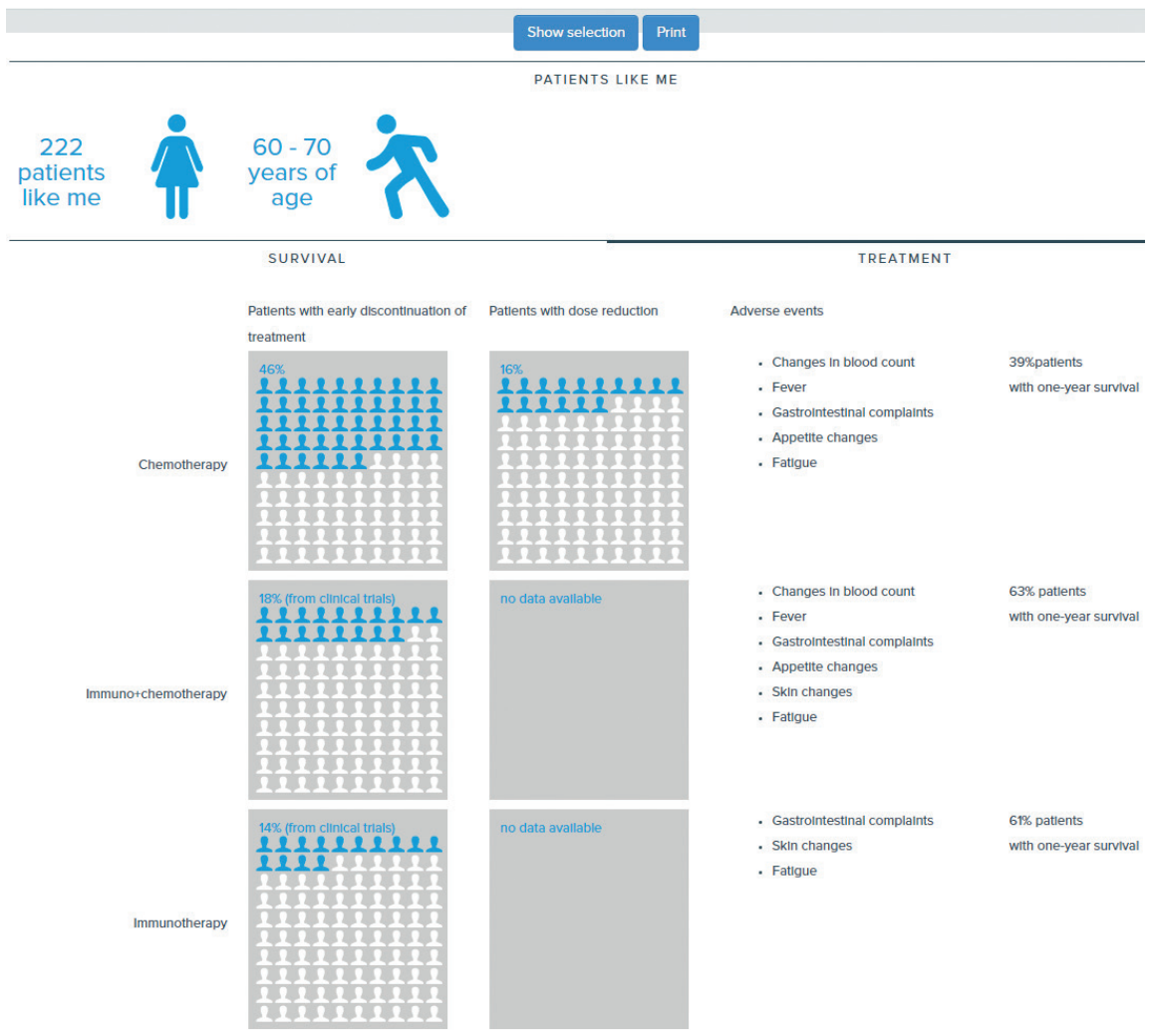

Figure 3. Screen shot of web-based tool for stage IV NSCLC patients - part C

reported an added value of the web-based tool when used in clinical practice. However, some patients stated that the tool was not useful to them, because "most of the information was already explained by the thoracic oncologist". Overall, 8 of 17 patients (47\%) stated the tool was useful in decision-making. Several patients reported that they already had made a treatment decision, varying from "Actually, I already made the decision myself. Doing nothing is not an option. I'll go for it!" to "I don't want chemotherapy and there are no treatment options for me which give curation". From some, it seemed that the tool still helped in confirming their treatment decision.

Two patients mentioned areas for improvement: adding reasons for early discontinuation on the tab about toxicities and side effects (part $\mathrm{C}$ of the tool); adding real-world outcomes for novel treatment options (i.e. immunotherapy); and some visual challenges when using the print option. 


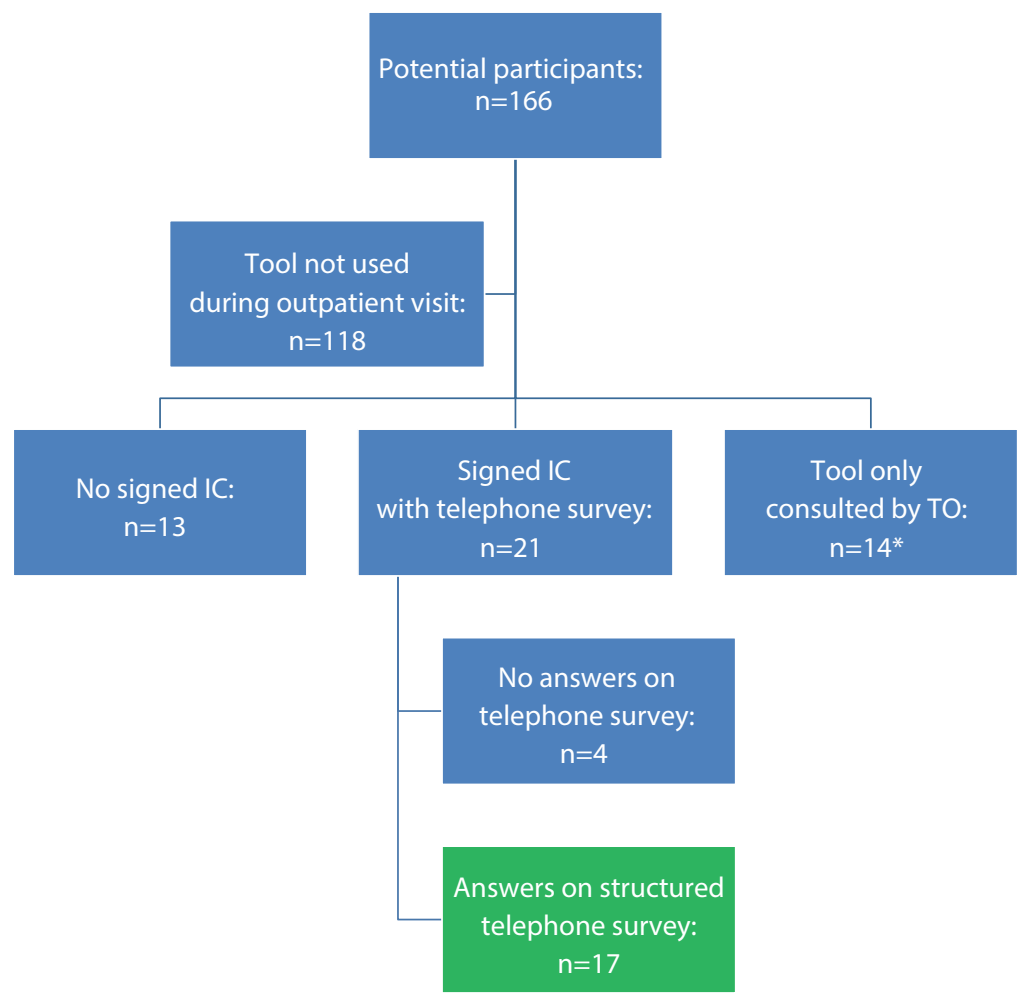

Figure 4. Flowchart of participants towards the telephone survey. * of which 2 patients declined to look into the information in the web-based tool during consultation. TO: thoracic oncologists; IC: informed consent

\section{Added value of the tool according to TOs}

The concept of the web-based tool seemed to be supported by TOs, however, a limited amount of time was spent for actual use of the tool in clinical practice. One TO noted that "I used the tool less often in clinical practice than expected/hoped", which seems to confirm the general tendency observed in our study. The questionnaire for TOs, which was completed by 10 respondents, revealed some barriers for use of the tool during consultation of patients. The most given reason was that the information in the web-based tool was considered alarming and confronting to patients. Additionally, TOs reported that patients declined to look into the information as displayed in the web-based tool. Furthermore, TOs stated that the tool rarely changed treatment advice. Lastly, the lack of real-world outcomes about immunotherapy was often mentioned as barrier for using the tool in clinical practice. 
Table 1. Characteristics of participants in telephone survey

\begin{tabular}{lc}
\hline & $\begin{array}{c}\text { Participants in telephone survey } \\
(\mathbf{n}=\mathbf{1 7})\end{array}$ \\
\hline Gender & $12(71 \%)$ \\
Male & $5(29 \%)$ \\
Female & $66(51-80)$ \\
Age (median (range)) & \\
ECOG PS & $15(88 \%)$ \\
$0-1$ & $1(6 \%)$ \\
$\geq 2$ & $1(6 \%)$ \\
Missing & \\
Histology & $4(24 \%)$ \\
Squamous & $12(71 \%)$ \\
Non-squamous & $1(6 \%)$ \\
Missing & \\
PD-L1 expression & $9(53 \%)$ \\
$<1 \%$ & $3(18 \%)$ \\
$1-49 \%$ & $3(18 \%)$ \\
$\geq 50 \%$ & $2(12 \%)$ \\
Missing & \\
\hline
\end{tabular}

ECOG PS: Eastern Cooperative Oncology Group performance status; PD-L1: programmed death-ligand 1.

Despite all barriers, $83 \%$ of the TOs reported an added value of using the web-based tool in clinical practice, because "the tool gives transparent and less abstract information on treatment outcomes for patients" and "the tool serves as a proper framework for giving information to patients". According to the TOs, this contributes to realistic expectations about the pros and cons of a treatment and enables informed decision-making.

\section{Decisional roles}

Preferred and used decisional roles during consultation were different according to patients and TOs (Table 2). We collapsed the original five categories of the CPS into three (i.e., 'active role', 'collaborative role', and 'passive role') according to Degner'10. Only a quarter of the patients $(n=4,24 \%)$ expressed their preference for the doctor and patient sharing responsibility for the decision about which treatment was best, while most of the TOs (60\%) reported that shared responsibility with their patients for treatment decision is preferred. More than half of the patients (53\%) preferred to make the final treatment decision themselves ('active role'), where only 30\% of the TOs preferred an active role for patients ('passive role' for themselves). 
Table 2. Preferred and used decisional roles according to patients and TOs $\left(\right.$ CPS $_{\text {post }}$ and CPS)

\begin{tabular}{lcc}
\hline $\begin{array}{l}\text { Preferred or used decisional } \\
\text { role during consultation }\end{array}$ & $\begin{array}{c}\text { Patients, CPS } \\
(\mathbf{n}=\mathbf{1 7})\end{array}$ & $\begin{array}{c}\text { Thoracic oncologists, CPS } \\
(\mathbf{n}=\mathbf{1 0})\end{array}$ \\
\hline Passive role & $4(24 \%)$ & $3(30 \%)$ \\
Collaborative role & $4(24 \%)$ & $6(60 \%)$ \\
Active role & $9(53 \%)$ & $1(10 \%)$ \\
\hline
\end{tabular}

CPS: Control Preferences Scale

\section{DISCUSSION}

We developed a web-based tool for patients with stage IV NSCLC and their TOs which provides real-world outcome data from available treatment options. The tool provided insight on both treatment outcomes from patients resembling selected characteristics and trial efficacy data for reference. The majority of patients and TOs reported an added value in terms of usefulness and appreciation of the web-based tool when used in clinical practice.

To our knowledge, this is the first study that developed and evaluated a tool that provides real-world outcomes data to patients with metastatic NSCLC and their oncologists. Other tools we know of are constructed based on trial efficacy data solely. In contrast, we constructed a unique interface wherein individual real-world outcomes are shown together with an aggregated trial efficacy result so that patients and TOs can observe how an individual patient fits to the population in the trial. Besides this, the tool provided data about how frequent what treatment (or best supportive care) was started in past patients. Although our tool fulfilled the wished-for function of integrating knowledge from clinical trials and real-world outcomes data, the tool was still infrequently used in clinical practice. Explanations from our evaluation are that real-world outcomes of recently introduced treatment options (immunotherapy) were lacking and that the information was considered confronting to patients. Additionally, preferred and used roles regarding treatment decisions during consultation were different according to patients and TOs.

Besides strengths, our study also has limitations. First, the evaluation can be considered limited because of sub-optimal implementation in clinical practice, reflected in the 50\% adoption rate and $29 \%$ usage rate. This hampers generalizability of statements about the added-value of the tool. Although TOs were strongly involved in the design of the web-based tool, actually making use of the tool in clinical practice was considered complex. 
In an observational study on communication about life expectancy with advanced cancer patients, Henselmans et al.12 found that oncologists did not initiate talk about life expectancy easily as they were generally not trained or encouraged regarding prognostic communication. Education on skills, knowledge and attitudes towards well-informed decision-making in combination with how to use the tool in clinical practice might be a way to improve this. Second, real-world data from recently introduced treatment options (mono immuno therapy and chemo-immunotherapy combinations) were not yet available from the hospitals. As a solution for this, in the tool, we predicted these outcomes by multiplying aggregated trial efficacy data from these novel treatments by the mean efficacy-effectiveness factor $^{3}$ from the patients resembling the selected criteria. We acknowledge, however, that this is suboptimal and has caused some TOs to be less convinced to use the tool with patients.

Third, bias may have been introduced by the fact that less than half of the patients who experienced the tool participated in the telephone survey. It is not inconceivable that our evaluation is driven by patients being above average positive about well-informed decision-making. On the other hand, the mean age, and distribution of gender, ECOG PS and histology in our sample was comparable to patients not being interviewed and also to patients from a large unselected population of patients with metastatic NSCLC. ${ }^{3}$

Despite the limitations above, our study provides important results upon which to base further work to improve well-informed treatment decisions based on real-world data. First, our evaluation learned that for adaptation by TOs, tooling must evolve and reflect advances in treatment strategies. A way to organise this, is to incorporate decision support systems in electronic health records in combination with smart algorithms, such that continuous updating and rapid adaptation of these instruments are ensured. ${ }^{5}$ Second, more effort should be invested in guiding implementation coupled with training in shared decision-making.

\section{ACKNOWLEDGEMENTS}

We want to thank all patients who participated in the semi-structured interviews and telephone surveys. Furthermore, we want thank all thoracic oncologists and other health care professionals mentioned below for participating in the focus group and other group meetings, and for using the tool in practice, reaching patients for interviews, performing informed consent procedures, and participating in the questionnaire for TOs.

- St. Antonius Hospital, Nieuwegein/Utrecht, The Netherlands: F.M.N.H. Schramel, E.A. Kastelijn, S.W.A. van Haarlem, J.G.M. Ribbert, J.C. de Bree, J.H.M. Dernison

- Canisius-Wilhelmina Hospital, Nijmegen, The Netherlands: L.C. Vermeer, M. Zijlker, Y. Berk, J. van der Mee 
- Martini Hospital, Groningen, The Netherlands: W. Jacobs, J.W.G. van Putten, M.K. Witteveen

- OLVG, Amsterdam, The Netherlands: P.W.A. Kunst, A.A.J. Smit, T. Lammers

- Catharina Hospital, Eindhoven, The Netherlands: B.E.E.M. van den Borne, J. Curfs

- Medisch Spectrum Twente, Enschede, The Netherlands: J.H. Schouwink. 


\section{REFERENCES}

1. Phillips CM, Parmar A, Guo H, et al: Assessing the efficacy-effectiveness gap for cancer therapies: A comparison of overall survival and toxicity between clinical trial and population-based, real-world data for contemporary parenteral cancer therapeutics. Cancer 126:1717-1726, 2020

2. Templeton AJ, Booth CM, Tannock IF: Informing Patients About Expected Outcomes: The Efficacy-Effectiveness Gap. J Clin Oncol 38:1651-1654, 2020

3. Cramer-van der Welle CM, Peters BJM, Schramel F, et al: Systematic evaluation of the efficacy-effectiveness gap of systemic treatments in metastatic nonsmall cell lung cancer. Eur Respir J 52, 2018

4. Stacey D, Legare F, Col NF, et al: Decision aids for people facing health treatment or screening decisions. Cochrane Database Syst Rev:CD001431, 2014

5. Revesz D, Engelhardt EG, Tamminga JJ, et al: Needs with Regard to Decision Support Systems for Treating Patients with Incurable Non-small Cell Lung Cancer. J Cancer Educ 35:345-351, 2020

6. Revesz D, Engelhardt EG, Tamminga JJ, et al: Decision support systems for incurable non-small cell lung cancer: a systematic review. BMC Med Inform Decis Mak 17:144, 2017

7. Al-Itejawi HH, van Uden-Kraan CF, Vis AN, et al: Development of a patient decision aid for the treatment of localised prostate cancer: a participatory design approach. J Clin Nurs 25:1131-44, 2016

8. Melissant HC, Verdonck-de Leeuw IM, Lissenberg-Witte Bl, et al: 'Oncokompas', a web-based self-management application to support patient activation and optimal supportive care: a feasibility study among breast cancer survivors. Acta Oncol 57:924-934, 2018

9. Rodenburg-Vandenbussche S, Pieterse AH, Kroonenberg PM, et al: Dutch Translation and Psychometric Testing of the 9-Item Shared Decision Making Questionnaire (SDM-Q-9) and Shared Decision Making Questionnaire-Physician Version (SDM-Q-Doc) in Primary and Secondary Care. PLoS One 10:e0132158, 2015

10. Degner LF, Sloan JA, Venkatesh P: The Control Preferences Scale. Can J Nurs Res 29:21-43, 1997

11. Kelders SM, Kok RN, Ossebaard HC, et al: Persuasive system design does matter: a systematic review of adherence to web-based interventions. J Med Internet Res 14:e152, 2012

12. Henselmans I, Smets EMA, Han PKJ, et al: How long do I have? Observational study on communication about life expectancy with advanced cancer patients. Patient Educ Couns 100:1820-1827, 2017 


\section{APPENDICES CHAPTER 8}

\section{APPENDIX 1 TELEPHONE SURVEY FOR PATIENTS}

Questions asked during the telephone survey for patients were partly based on the Satisfaction with Cancer Information Profile (SCIP) questionnaire1, regarding satisfaction with the tool and timing of information, and, to a lesser extent, the PrepDM questionnaire?, regarding preparation for making a decision. A combination of questions derived from these questionnaires were used, but without official scoring on items and scales, and complemented with general questions about the web-based tool. Explanations were asked to better contextualize the given answers.

The telephone survey ended with the CPS post as described by Rodenburg-Vandenbussche ${ }^{3}$. The original CPS was developed by Degner et al. ${ }^{4}$ and presents subjects with a choice of five decisional roles and requires them to identify the one that best describes their preferred position. The CPS post is supposed to evaluate the experienced role in the final treatment decision after a consultation on a five-point Likert scale, which has a good reliability and validity ${ }^{3}$.

The following questions were asked during the telephone survey for patients:

- How understandable was the web-based tool and information given to you?

- How useful was the information in the web-based tool to you?

- Did the tool help you to make a decision about your treatment?

- How would you rate the timing at which you received information through the tool?

- In your opinion, are there any areas for improvement considering the web-based tool?

- Is there anything you would like to note about the decision tool or about your treatment decision in general?

- In general: did the tool have added value for you when communicating with your thoracic oncologist at the consultation visit? With other words: was the tool overall useful and did you appreciate it?

- Can you identify which of the following descriptions best describes your treatment decision made during the consultation?

1: I made the decision alone

2: I made the decision alone considering my physician's opinion

3: I shared the decision with my physician

4: My physician decided, considering my opinion

5: My physician made the decision

1 Llewellyn, C.D., et al., Development and preliminary validation of a new measure to assess satisfaction with information among head and neck cancer patients: the satisfaction with cancer information profile (SCIP). Head Neck, 2006. 28(6): p. 540-8

2 Bennett, C., et al., Validation of a preparation for decision making scale. Patient Educ Couns, 2010. 78(1): p. 130-3.

3 Rodenburg-Vandenbussche, S., et al., Dutch Translation and Psychometric Testing of the 9-Item Shared Decision Making Questionnaire (SDM-Q-9) and Shared Decision Making Questionnaire-Physician Version (SDM-Q-Doc) in Primary and Secondary Care. PLoS One, 2015. 10(7): p. e0132158.

4 Degner, L.F., J.A. Sloan, and P. Venkatesh, The Control Preferences Scale. Can J Nurs Res, 1997. 29(3): p. 21-43. 


\section{APPENDIX 2 SCREENSHOTS WEB-BASED TOOL IN DUTCH}

\section{Beslishulp longkanker}

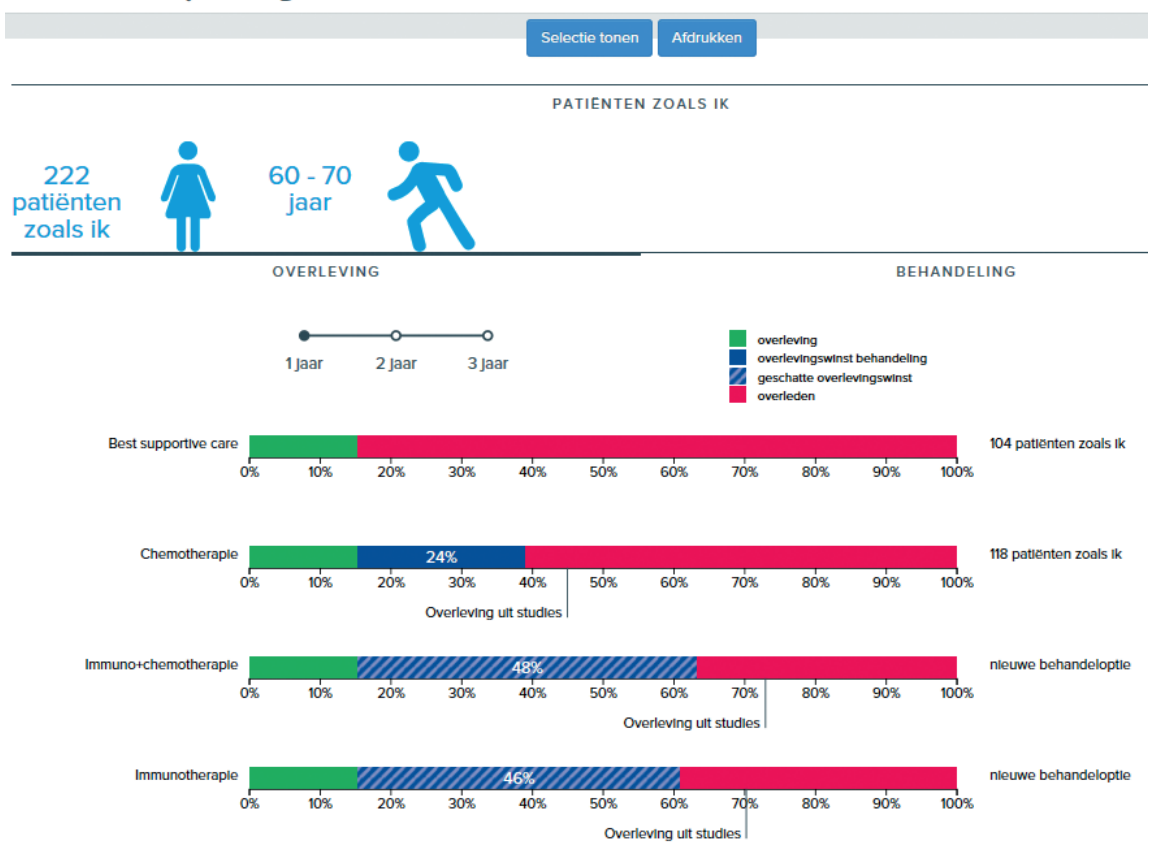

SFigure 1. Screenshot part B of original Dutch interface: tab with graphical representation of survival outcomes 


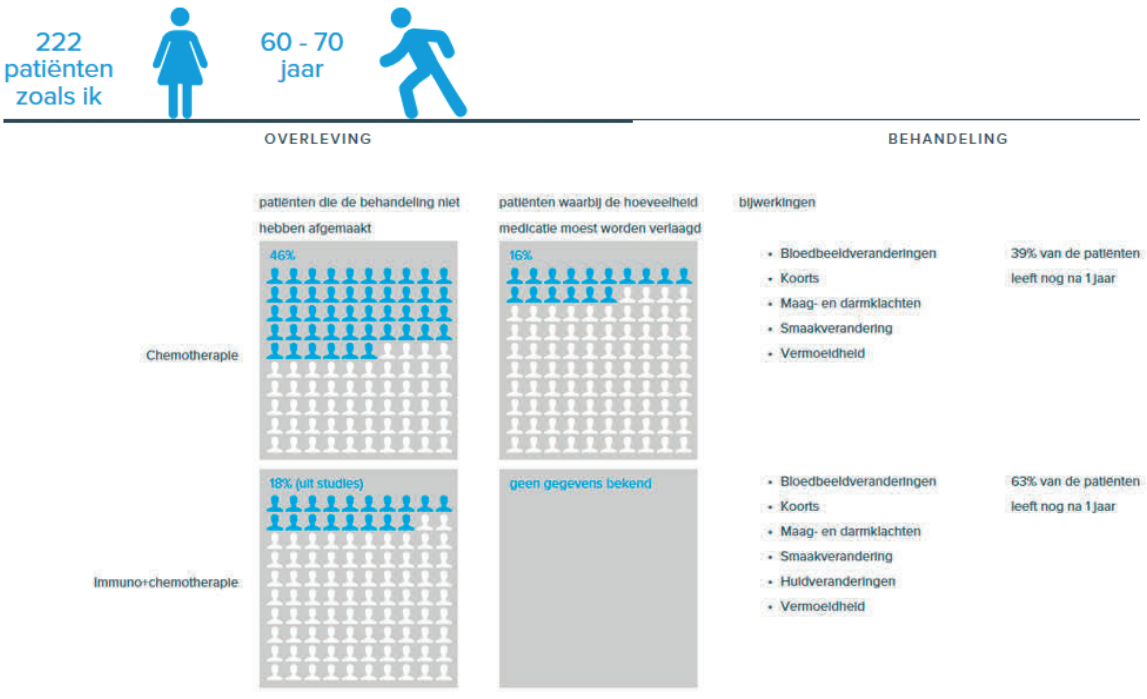

SFigure 2. Screenshot part C of original Dutch interface: tab with graphical representation of toxicities and side effects 


\section{APPENDIX 3 DETAILS ON DEVELOPMENT OF PART B AND C OF THE WEB-BASED TOOL}

\section{Part B - graphical representation of survival outcomes}

Instead of individual survival points, one-year, two-year and three-year survival rates based on real-world data from our earlier described cohort ${ }^{1}$ were shown for chemotherapy, targeted therapy, and best supportive care, adjusted for selected patient characteristics (gender, age and ECOG PS). These survival rates were presented in the tool with a bar chart with percent format, where the gain in survival from potential treatment options is shown relative to the survival rate of best supportive care. Additionally, pooled outcomes from the systematic review of efficacy data from clinical trials $5^{5}$ were shown in the bar charts for reference. For novel treatment options (mono immunotherapy, and immuno-chemotherapy combinations), such a reference value was calculated likewise and shown in the respective bar charts. Because of lacking real-world data on these new treatments, estimated outcomes were presented using individual efficacy-effectiveness (EE) factors ${ }^{1}$ from the resembling patients. For example, if a selected patient (based on age, gender, and ECOG PS) had an EE factor of 0.7 for platinum-doublet therapy, the expected outcome for platinum-doublet plus immunotherapy was estimated at 0.7 times the reference value/ outcome for the latter treatment from clinical trials as well. The individual expected outcomes where combined to present the expected survival rate for the total group of patients with the chosen patient characteristics.

\section{Part C - graphical representation of treatment outcomes}

In part $\mathrm{C}$ of the tool (i.e. tab about toxicities and side effects) proxies for toxicity are limited to the graphical representation of percentage of real-world patients with dose reduction ( $\leq 80 \%$ of the initial dose) and early discontinuation (<4 cycles), besides textual information on most common side effects (maximum of five per treatment option). Because these outcome data is presented on an individual tab, the corresponding survival rates per treatment option were represented again in part C (in text) for complete provision of available information on real-world outcomes.

1 Cramer-van der Welle CM, Peters BJM, Schramel F, et al: Systematic evaluation of the efficacy-effectiveness gap of systemic treatments in metastatic nonsmall cell lung cancer. Eur Respir J 52, 2018 

General discussion 

This thesis addresses the gap between clinical outcomes of patients in real-world and the outcomes of patients in clinical trials of systemic treatments for metastasized lung cancer. We showed that results from RCTs are not easily generalizable to clinical practice. Furthermore, knowledge about real-world outcomes can support both clinical decision-making and discussions about the added value of novel treatment options.

\section{EFFICACY-EFFECTIVENESS GAP}

This thesis shows that patients with metastasized lung cancer treated in real-world practice have a significantly shorter survival than those in clinical trials. The pattern of reduced effectiveness and more toxicity that we observed on an absolute scale, irrespective of the type of first-line systemic treatment given (chemotherapy, targeted therapy or immunotherapy) and the type of lung cancer (NSCLC or SCLC), adds to the perception that the external validity of clinical trial results is limited.

An important explanatory factor for the EE gap from our data is a negative association between a higher/worse ECOG performance status (PS) and overall survival in patients with first-line chemotherapy for NSCLC or SCLC (Chapter 3 and Chapter 7, respectively) or second-line immunotherapy in NSCLC (Chapter 5). This confirms the general thinking that in clinical trials patients with worse ECOG PS are excluded while in regular practice these patients are offered treatment as well, resulting in an EE gap. A review by Dall'Olio, et al. [1] showed that ECOG PS $\geq 2$ at the beginning of the treatment holds prognostic significance in patients treated with immunotherapy for NSCLC, as for chemotherapy and targeted agents. Additionally, as Templeton et al. stated [2], the goal of trials sponsored by the pharmaceutical industry is to select patients for whom there is most likely a benefit and there are least likely complications from a new drug. Such trials can lead to product registration for a target population similar to those recruited to the trial, but physicians may offer treatment to patients who do not completely match the eligibility criteria of the original clinical trial, particularly within the setting of limited treatment options [3]. However, differences in patient factors such as performance status is probably not the sole driver of the observed EE gap. ECOG PS was associated with worse outcomes in the real-world setting, however, it appeared to be equally prevalent in real-world compared to clinical trials concerning chemotherapy for NSCLC (Chapter 3), which means that other (unmeasured) factors are likely to be involved as well. The aetiology for the EE gap is believed to be multifactorial, with contributing factors including differences in patient populations, health care delivery, and variability in the experience of health care providers with specific treatments [3]. For example, participants in RCTs are treated according to guidelines more often and receive more in-hospital procedures [4]. Furthermore, participants in RCTs often are treated by specialized doctors in specialized centres, where there is close monitoring and guidance about management of adverse events [2]. 
Another explanation for the EE gap from our data could be the observed lower frequency of patients who received a subsequent line of therapy after chemotherapy or immunotherapy in the real-world population compared to clinical trial participants. Patients who received systemic therapy through participating in a clinical trial, for example, might have an above average intrinsic willingness to search for further treatment options after treatment failure. Other possible explanations could be that clinics active in trial enrolment communicate more about remaining experimental treatments, or that patient characteristics other than PS, limit the tolerability for subsequent systemic treatment to a larger extent in regular care patients. To illustrate, the retrospective study by Tiu et al. [5] evaluated patients with solid malignancy who were listed as ineligible for a clinical trial and found that the most common ineligibility reason leading to no subsequent treatment in previously treated patients were ECOG $\geq 2$, rapid disease progression, and abnormal liver function tests due to liver metastases.

Besides knowledge on efficacy, clinical trials play a crucial role in informing care for patients with cancer as their results shape the regulatory approval of new treatments and lead to the creation of treatment guidelines. In addition, oncologists use survival and toxicity data from clinical trials to counsel patients, inform decision-making, and obtain consent for treatment [3]. Our findings on the EE gap highlights the importance of using real-world data regarding clinical effectiveness and toxicity of systemic therapies to complement RCT data to inform patients about treatment options and potential outcomes. Our web-based tool for improving informed decision-making in the palliative setting provided insight on both treatment outcomes from patients resembling selected characteristics and trial efficacy data for reference. The majority of patients and thoracic oncologists reported an added value in terms of usefulness and appreciation of the tool when used in clinical practice (Chapter 8).

\section{IMPACT ON DRUG LIFE CYCLE}

Nowadays, most systemic treatments in oncology are studied with effect on PFS as primary study endpoint. Data about effects on OS are then provided after drug approval. Several reports have shown that effects on PFS often do not extend to similar effects on OS $[6,7]$. For some treatments this is not an issue when longer progression free times provide large gains in quality of live. Our study in Chapter 5 adds another dimension to the translation of PFS to OS. We observed that PFS does not differ between trial participants and patients in real-world, but that for OS there is a difference. This underlines that definite conclusions about the existence of an EE-gap should not be based on PFS alone. 
Regulatory agencies and payers might revisit initial approval when subsequent data from routine practice become available [2]. However, Kemp and Prasad [8] reported that for 18 of the 36 cancer drugs that were approved by the FDA from 2008 to 2012 on the basis of surrogate outcomes, post-market studies did not indicate clear benefit in OS; yet only one of these drugs has had its marketing authorization removed. These findings advocate the incorporation of mature evidence and expanded use into reimbursement decisions. Additionally, the observed shorter OS time after first-line chemotherapy or immunotherapy in real-world could provide an argument to rework the initial clinical trial-based cost-effectiveness assessment, since the models used for estimating cost-effectiveness have made assumptions in translating the gain in median PFS to OS. In Chapter 5, we demonstrate that these assumptions do not always hold.

\section{USE OF REAL-WORLD DATA IN DRUG LIFE CYCLE}

For most newly approved treatments, the clinical evidence is not sufficient to guide physicians and policy makers in choosing the optimal treatment for their patients [9]. Strategies to incorporate real-world evidence earlier into the drug life cycle, for example, pragmatic trials, are being developed. Pragmatic trials inform a clinical or policy decision by providing evidence for adoption of the intervention into real-world clinical practice [10]. As Zuidgeest et al. stated [9], evidence from pragmatic trials is specifically relevant when treatment options do already exist for the disease under study and when the real-life situation is expected to influence the treatment effect, as in current practice for patients with metastasized lung cancer.

Another strategy is to integrate research (including pragmatic trials) and care in learning health care systems (LHSs), where health care activities and data from various sources will be continuously analysed, learned from, and improved [11-13]. The Santeon hospitals created the Santeon Farmadatabase infrastructure as an important first step towards implementing a LHS in the direction of pharmacotherapy [14]. Chapter 3, 5 and 7 showed that such an infrastructure can be linked to relevant patient characteristics and outcome data in lung oncology collected in other Santeon databases, to evaluate the value of treatment options in clinical practice. Furthermore, in Chapter 4, we were able to link drug costs of systemic treatment per patient based on the milligrams of drugs from the Santeon Farmadatabase to the corresponding OS, to report on trends in systemic treatment costs and OS achieved in consecutive years.

Additionally, a LHS provides opportunities for regular update of data in instruments for clinical decision-making, such as our web-based tool for patients with metastasized NSCLC and their thoracic oncologists. From Chapter 8, we learned that for adaptation of 
such a tool by thoracic oncologists, tooling must evolve and reflect advances in treatment strategies. A way to organise this, is to incorporate decision support systems in electronic health records in combination with smart algorithms, such that continuous updating and rapid adaptation of these instruments are ensured [15]. To illustrate, Finlayson et al. [16] developed the Melanoma Rapid Learning Utility which can provide real-time, data-driven clinical decision support for melanoma treatment. It recapitulated known biomedical knowledge about melanoma treatment, showed promise for clinical utility when used by oncologists, is extensible to other types of cancer, and can incorporate more and richer data in the future.

Patient registries and electronic health records are established examples of real-world data sources, but other potential sources could be useful to contribute as a supplementary source of evidence to assess effectiveness in real-world as well. For example, data derived from applications on mobile devices or health data reported by patients could enrich real-world data. Kalf et al. [17] conducted an explorative review to identify examples in oncology where health data were collected using social media. It appears that social media may be a potential source of real-world data, particularly on aspects such as adverse events, occurrence of disease-specific symptoms, adherence behaviour, and quality of life. Such information could have enhanced our studies regarding explanatory factors for lower effectiveness of chemotherapy and immunotherapy in real-world. Furthermore, data on quality of life and adverse events could have enriched our tool for improving well-informed treatment decisions.

\section{RELEVANCE AND LIMITATIONS OF OUR WORK}

In the past years, the use of real-world data and subsequently research into the efficacy-effectiveness gap for systemic therapies in oncology is becoming increasingly important and innovative. Our studies illustrate that observational (real-world) and randomized studies can be complementary.

Our study as described in Chapter 3 was the first that provided a complete overview on the EE gap for different systemic treatments (chemotherapy and targeted therapy) in a large, unselected real-world population of metastatic NSCLC patients, with a very low number of missing values. At that time, only few single treatment comparisons were made between outcomes in clinical trials and in real-world.

Our introduction of the efficacy-effectiveness factor created insight in how an individual outcome relates to the median OS from the corresponding clinical trial, and could be subsequently used to generate an overview of the divergence between efficacy in trials and effectiveness of treatment regimens in real-world. Furthermore, patient characteristics associated with a possible EE gap were explored. This method could be easily applied to 
assess the EE gap of systemic treatments in patients with extensive disease SCLC (Chapter 7), and likely in other types of cancer. However, a restriction of this method is the requirement of mature survival data where nearly all patients have reached their endpoint.

Additionally, a recurring limitation of our studies relates to the approach to compare median OS between real-world and clinical trials primarily. An alternative way could be a hazard ratio based approach, however, this was not feasible because of the unavailability of individual patient data (IPD) from clinical trials. The absence of IPD also inhibited a multivariable Cox regression in the search for explanatory factors. Unfortunately, IPD from past clinical trials were not available in the public domain for this type of analyses.

This limitation became relevant again when assessing the EE gap for immunotherapy in NSCLC later on (Chapter 5). Many studies had shown that the shape of the survival curve for immunotherapy was different than for chemotherapy, with some extra early deaths with immunotherapy and a plateau of long-term survivors later on. Therefore, to assess the real-world effectiveness of immune checkpoint inhibitors (ICIs), survival curves had to be compared to detect potential differences between survival dynamics in real-world and trials. We found a method to assess hazard ratios (HRs) between real-world immunotherapy treatment regimens and groups in corresponding RCTs to compare OS and PFS by digitizing the Kaplan-Meier (KM) curves for the immunotherapy arm from the included clinical trials and reconstructed this data as IPD [18]. Both the method using EE factors, and the method to reconstruct IPD data from KM curves showed a significant EE gap for first-line pembrolizumab. Unfortunately, (patient) factors potentially responsible for an EE gap in immunotherapy could only be analysed on the cohort level instead of patient level, because these IPD from the respective clinical trials were not available.

Therefore, we highly recommend to make IPD available from clinical trials to compare PFS and OS between real-world and clinical trials, and to be able to search for explanatory factors for any EE gap.

\section{FUTURE PERSPECTIVES}

This thesis provides important results upon which to base further work to improve the use of evidence from real-world studies in healthcare decision-making. Our findings advocate the incorporation of mature evidence and expanded use from real-world into the drug life cycle. Therefore, it is recommended from our studies to improve the interoperability and the exchange of electronic health information between different sources available for research, which is also one of the recommendations identified by ASCO to enhance the role of observational research in clinical decision-making [19]. Several researchers and stakeholders also advocate criteria for assessment of validity and the need for transparency of the research process in studies using real-world evidence to enable decision-makers to 
evaluate the quality of the methods used and the applicability of the evidence that results from those studies [20-23]. Examples are study registration, information on operational and design decisions, replicability, and stakeholder involvement. Such criteria provide a trustworthy foundation for the expanded use of real-world evidence in health care decision-making [20].

Furthermore, besides adequate exchange of data, the data capturing in our studies could have been improved by real-time health information. With real-time data from national registries and patients' medical records from the Santeon hospitals, routine clinical data collection could be combined with clinical decision-support algorithms. Such a combination allows professionals to translate novel insights derived from the treatment of previous patients directly into patient management, which in turn yields data that can be used to evaluate and fine-tune clinical decisions in the future [24]. This approach can support future evolvement of our web-based tool for patients and thoracic oncologists regarding continuous updating and early reflection of advances of novel treatment strategies.

Besides use of real-world data, outcomes from clinical trials remain required. As Ramamoorthy et al. [25] stated: just like how RCTs cannot answer all the questions, real-world data cannot address all the questions either. Future research on the EE gap and explanatory factors can be supported by individual patient data from RCTs in combination with real-world data from, for example, learning healthcare systems.

\section{FINAL CONCLUSION}

The studies reported in this thesis provide evidence for a gap between efficacy of systemic treatments for metastasized lung cancer as reported in clinical trials and effectiveness in real-world clinical practice. The gap in overall survival was observed for all types of systemic treatment options (chemotherapy, targeted therapy, and immunotherapy). Differences in ECOG PS and extent of proceeding to subsequent lines of treatment are important drivers of this gap. Availability of IPD from RCTs could facilitate the search for further explanatory factors for the EE gap in the field of cancer treatments in order to optimize the benefit/risk balance for specific groups of patients.

Finally, patients and thoracic oncologists appreciated the web-based tool in clinical practice, which was developed to support well-informed treatment decisions based on real-world treatment outcomes with trial efficacy data for reference. 


\section{REFERENCES}

1. Dall'Olio, F.G., et al., ECOG performance status $>/=2$ as a prognostic factor in patients with advanced non small cell lung cancer treated with immune checkpoint inhibitors-A systematic review and meta-analysis of real world data. Lung Cancer, 2020. 145: p. 95-104.

2. Templeton, A.J., C.M. Booth, and I.F. Tannock, Informing Patients About Expected Outcomes: The Efficacy-Effectiveness Gap. J Clin Oncol, 2020. 38(15): p. 1651-1654.

3. Phillips, C.M., et al., Assessing the efficacy-effectiveness gap for cancer therapies: A comparison of overall survival and toxicity between clinical trial and population-based, real-world data for contemporary parenteral cancer therapeutics. Cancer, 2020. 126(8): p. 1717-1726.

4. Kennedy-Martin, T., et al., A literature review on the representativeness of randomized controlled trial samples and implications for the external validity of trial results. Trials, 2015. 16: p. 495.

5. Tiu, C., et al., Effect of Reasons for Screen Failure on Subsequent Treatment Outcomes in Cancer Patients Assessed for Clinical Trials. Oncology, 2019. 97(5): p. 270-276.

6. Mushti, S.L., F. Mulkey, and R. Sridhara, Evaluation of Overall Response Rate and Progression-Free Survival as Potential Surrogate Endpoints for Overall Survival in Immunotherapy Trials. Clin Cancer Res, 2018. 24(10): p. 2268-2275.

7. Tan, A., et al., Differences in Treatment Effect Size Between Overall Survival and Progression-Free Survival in Immunotherapy Trials: A Meta-Epidemiologic Study of Trials With Results Posted at ClinicalTrials.gov. J Clin Oncol, 2017. 35(15): p. 1686-1694.

8. Kemp, R. and V. Prasad, Surrogate endpoints in oncology: when are they acceptable for regulatory and clinical decisions, and are they currently overused? BMC Med, 2017. 15(1): p. 134.

9. Zuidgeest, M.G.P., et al., Series: Pragmatic trials and real world evidence: Paper 1. Introduction. J Clin Epidemiol, 2017. 88: p. 7-13.

10. Ford, I. and J. Norrie, Pragmatic Trials. N Engl J Med, 2016. 375(5): p. 454-63.

11. Medicine, I.o., The Learning Healthcare System: Workshop Summary, ed. L. Olsen, D. Aisner, and J.M. McGinnis. 2007, Washington, DC: The National Academies Press. 374

12. Tuzzio, L. and E.B. Larson, The Promise of Pragmatic Clinical Trials Embedded in Learning Health Systems. EGEMS (Wash DC), 2019. 7(1): p. 10.

13. Palin, V., et al., Developing the infrastructure to support the optimisation of antibiotic prescribing using the learning healthcare system to improve healthcare services in the provision of primary care in England. BMJ Health Care Inform, 2020. 27(1).

14. van de Garde, E.M.W., et al., Pharmacotherapy within a learning healthcare system: rationale for the Dutch Santeon Farmadatabase. Eur J Hosp Pharm, 2019. 26(1): p. 46-50.

15. Revesz, D., et al., Needs with Regard to Decision Support Systems for Treating Patients with Incurable Non-small Cell Lung Cancer. J Cancer Educ, 2020. 35(2): p. 345-351.

16. Finlayson, S.G., et al., Toward rapid learning in cancer treatment selection: An analytical engine for practice-based clinical data. J Biomed Inform, 2016. 60: p. 104-13.

17. Kalf, R.R., et al., Use of Social Media in the Assessment of Relative Effectiveness: Explorative Review With Examples From Oncology. JMIR Cancer, 2018. 4(1): p. e11.

18. Guyot, P., et al., Enhanced secondary analysis of survival data: reconstructing the data from published Kaplan-Meier survival curves. BMC Med Res Methodol, 2012. 12: p. 9.

19. Visvanathan, K., et al., Untapped Potential of Observational Research to Inform Clinical Decision Making: American Society of Clinical Oncology Research Statement. J Clin Oncol, 2017. 35(16): p. 1845-1854.

20. Berger, M.L., et al., Good practices for real-world data studies of treatment and/or comparative effectiveness: Recommendations from the joint ISPOR-ISPE Special Task Force on real-world evidence in health care decision making. Pharmacoepidemiol Drug Saf, 2017. 26(9): p. 1033-1039.

21. Orsini, L.S., et al., Improving transparency to build trust in real-world secondary data studies for hypothesis testing-Why, what, and how: recommendations and a road map from the real-world evidence transparency initiative. Pharmacoepidemiol Drug Saf, 2020. 29(11): p. 1504-1513.

22. Patorno, E., S. Schneeweiss, and S.V. Wang, Transparency in real-world evidence (RWE) studies to build confidence for decision-making: Reporting RWE research in diabetes. Diabetes Obes Metab, 2020. 22 Suppl 3: p. 45-59. 
23. Wang, S.V., et al., Reporting to Improve Reproducibility and Facilitate Validity Assessment for Healthcare Database Studies V1.0. Pharmacoepidemiol Drug Saf, 2017. 26(9): p. 1018-1032.

24. Wouters, R.H.P., et al., Learning health care systems: Highly needed but challenging. Learn Health Syst, 2020. 4(3): p. e10211.

25. Ramamoorthy, A. and S.M. Huang, What Does It Take to Transform Real-World Data Into Real-World Evidence? Clin Pharmacol Ther, 2019. 106(1): p. 10-18. 
GENERAL DISCUSSION | 211 

Summary 

Results from clinical trials, although providing critical evidence of clinical activity, may not be reflective of treatment benefit and risk in all patient populations that will actually be treated in real-world practice. In this thesis, we assessed how clinical outcomes in real-world (effectiveness) correspond to outcomes in clinical trials (efficacy) of systemic treatments for metastasized lung cancer, which factors explained an efficacy-effectiveness (EE) gap, and we developed and evaluated a web-based tool in which we provide real-world outcomes to patients and thoracic oncologists (TOS).

\section{REAL-WORLD OUTCOMES OF PATIENTS WITH STAGE IV NON-SMALL CELL LUNG CANCER (NSCLC)}

Chapter 1 describes the development of a set of outcome indicators for lung cancer in the project 'Care for Outcomes' (CfO) of the Santeon hospitals. A literature review followed by expert review formed the basis for the indicator selection. The final set was tested in data of 5922 patients diagnosed with lung cancer (all types and stages) and appeared sensitive to detect differences in outcomes between hospitals in order to fuel discussions towards improvements in delivery of care.

In Chapter 2, an overview of day-to-day practice in the systemic treatment of stage IIIB/IV NSCLC and its clinical outcomes in six Santeon hospitals in the period 2008-2012 was given. Therefore, a retrospective observational cohort study with 2158 patients was conducted in the CfO registry. Of all patients, 48\% received systemic treatment and 34\% of those received second-line treatment. Treatment frequencies were different between age categories, disease stage, ECOG performance status (PS) and hospital $(p<0.001)$. Median survival was not different between hospitals or years of diagnosis.

Subsequently, in Chapter 3, the differences between outcomes in real-world and outcomes in clinical trials (EE gap) for patients with stage IV NSCLC, diagnosed between 2008 and 2014 in six Santeon hospitals, were assessed. For every patient, an efficacy-effectiveness (EE) factor was calculated by dividing individual patients' overall survival (OS) by the pooled median OS assessed from clinical trials with the respective treatment. From 2989 diagnosed patients, 1214 (41\%) started with first-line treatment. For all studied regimens, real-world OS was shorter than OS reported in clinical trials. Overall, the EE factor was 0.77 (95\% Cl 0.70-0.85; $p<0.001$ ), which means that survival of patients with metastatic NSCLC treated with chemotherapy or targeted therapy in real-world practice is nearly one-quarter shorter than for patients included in trials. Patients' performance status, earlier discontinuation and fewer subsequent lines of treatment partly explained this difference. 
In Chapter 4, changes in OS at the level of a diagnosis of stage IV NSCLC were compared with the development of systemic treatment costs over the years. Data on OS of 1214 patients from the study in Chapter 3 and amounts of drug units (milligrams) for every drug in the applied systemic cancer treatments until death from the Santeon Farmadatabase were collected. These amounts were multiplied by Dutch unit costs (Euros/mg) expressed in 2018 Euros to construct total drug costs per line of treatment per patient. Costs for day care visits were added for drugs requiring parenteral administration. Median OS and mean total drug costs showed no significant variation over the years. Mean total drug costs per 1 year of survival ranged from $€ 20,665$ to $€ 26,438$ during the period under study. Costs for first-line systemic treatment were significantly higher in 2011-2014 compared with 2008-2010.

As new treatment options emerged in more recent years, the EE gap of immunotherapy in patients with metastatic NSCLC diagnosed in 2015-2018 was studied in Chapter 5. Progressionfree survival (PFS) and OS from first-line pembrolizumab and second-line nivolumab were compared with clinical trial data by calculating hazard ratios (HRs). From 1950 diagnosed patients, 1005 (52\%) started with any first-line treatment, of which 83 received pembrolizumab. Nivolumab was started as second-line treatment in 141 patients. For both settings, PFS times were comparable between real-world and trials ( $H R \quad 1.08$ (95\% Cl 0.75-1.55), and HR 0.91 (95\% Cl 0.74-1.14), respectively). OS was significantly shorter in real-world for first-line pembrolizumab (median 15.8 vs. 30.0 months; HR 1.55 (95\%Cl 1.07-2.25)). Receiving subsequent lines of treatment was less frequent in real-world compared to trials.

\section{REAL-WORLD OUTCOMES OF PATIENTS WITH EXTENSIVE DISEASE (ED) SMALL CELL LUNG CANCER (SCLC)}

In Chapter 6, data on treatment patterns and survival in an unselected patient population with ED SCLC was presented. From 792 diagnosed patients with ED SCLC between 2008 and 2014 in six Santeon hospitals, 568 (72\%) started with first-line treatment. Of these patients, 41\% received second-line treatment. Only 68 patients received third-line treatment. For all treated patients, the mean age was 66 years. The majority (72\%) had an ECOG PS of 0 or 1 at diagnosis. Median OS of treated patients was 7.4 months.

Subsequently, in Chapter 7, the outcomes of the previous study were used to assess the EE gap of systemic treatments for ED SCLC. Overall, the median EE factor was 0.79 ( $p<0.001$ from 1.00), which implicates that the OS of patients with ED SCLC treated with systemic therapy in real-world practice is $21 \%$ shorter than for patients included in trials. Poor ECOG PS ( $\geq 2)$ and a higher age at diagnosis (age $\geq 65$ years) were independent predictors for a lower EE factor. The mean age and the proportion of patients with ECOG $\geq 2$ in real-world were different from those in clinical trials (mean age of 66 versus 62 years, and ECOG $\geq 2$ $25 \%$ versus $17 \%$; both $p<0.001$. 


\section{COMMUNICATION OF REAL-WORLD OUTCOMES TO PATIENTS}

Chapter 8 describes the development and evaluation of a web-based tool for patients with stage IV NSCLC and their TOs based on data of 2989 patients with stage IV NSCLC (from the study in Chapter 3) in a participatory design approach. This web-based tool shows treatments plus both real-world outcomes and clinical trial results after selecting patient characteristics ('patients like me'), to support informed clinical decision-making in the palliative setting. The tool was implemented and used by TOs in three of six Santeon hospitals (50\% adoption rate) and effectively used in 48 patients (29\% usage rate) of which 17 participated in a telephone survey. Ten TOs responded to a study specific questionnaire. Overall, the majority of these patients and TOs scored the tool as of added value (71\% and $83 \%$, respectively), which means that they consider the web-based tool as appreciated and useful in clinical practice.

In the General discussion, the use of real-world data and our results on the EE gap were discussed in a broader perspective of implications on drug life cycles. Furthermore, future perspectives were given. 


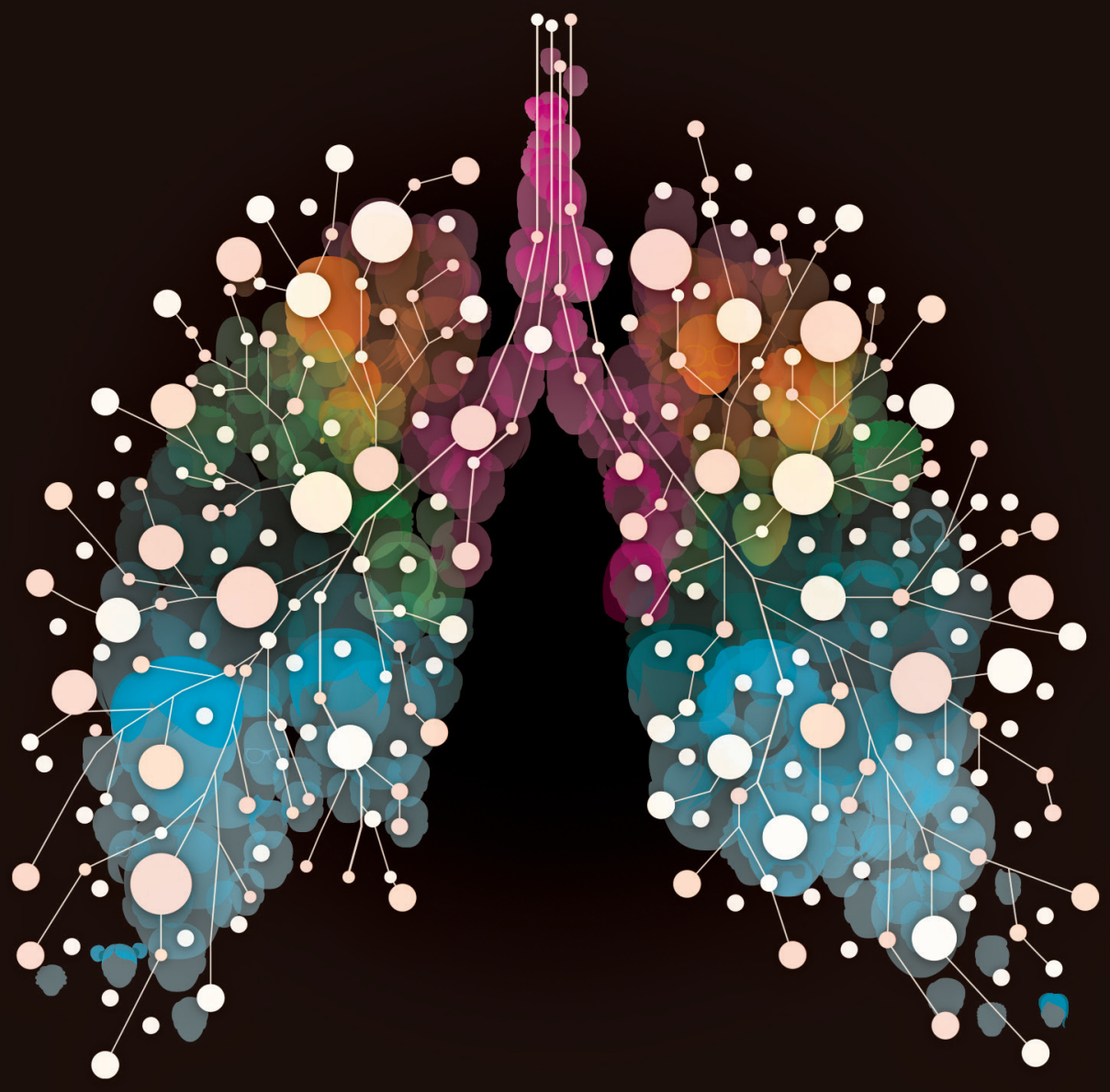


APPENDICES

Samenvatting

Dankwoord

Curriculum Vitae

List of publications 



\section{SAMENVATTING}

Hoewel klinische studies de werkzaamheid van geneesmiddelen goed kunnen aantonen, weerspiegelen deze onderzoeken mogelijk niet de resultaten van de behandeling bij alle (verschillende) patiënten die in de dagelijkse praktijk worden behandeld. In dit proefschrift is onderzocht of er een verschil is tussen uitkomsten in de dagelijkse praktijk (effectiviteit - effectiveness) en de resultaten uit klinische studies (werkzaamheid - efficacy) van diverse systemische behandelingen voor uitgezaaide longkanker. Daarnaast is onderzocht welke factoren een verschil tussen werkzaamheid en effectiviteit (efficacy-effectiveness gap; EE gap) kunnen verklaren. Tenslotte is een web-based tool voor patiënten en thoracale oncologen (TO's) ontwikkeld en geëvalueerd. Deze tool biedt inzicht in welke behandeling welke uitkomsten had bij vergelijkbare patiënten.

\section{Uitkomsten in de dagelijkse praktijk van patiënten met stadium IV niet-kleincellige longkanker (NSCLC)}

Hoofdstuk 1 beschrijft de ontwikkeling van een set uitkomstindicatoren voor longkanker in het project 'Zorg voor Uitkomst' (ZvU) van de Santeon ziekenhuizen. Een literatuuronderzoek gevolgd door een expertonderzoek vormden de basis voor de selectie van indicatoren. De uiteindelijke indicatorenset werd getest in data van 5922 longkanker patiënten (alle histologische typen en stadia). Hierbij werd duidelijk dat deze set verschillen in uitkomsten tussen ziekenhuizen kan detecteren én discussies over verbeteringen in de zorgverlening stimuleert.

In Hoofdstuk 2 wordt een overzicht gegeven van de klinische uitkomsten na systemische behandeling van patiënten met stadium IIIB/IV NSCLC uit zes Santeon ziekenhuizen in de periode 2008-2012. Hiervoor werd een retrospectieve observationele cohortstudie met 2158 patiënten uitgevoerd in de ZvU database. Van alle patiënten kreeg 48\% een systemische behandeling; $34 \%$ van deze patiënten onderging een vervolgbehandeling (zogenoemde tweedelijnsbehandeling). De behandelfrequenties waren verschillend tussen leeftijdscategorieën, ziektestadium, ECOG performance status (PS) en ziekenhuizen ( $p<0,001)$. De mediane overleving was niet verschillend tussen ziekenhuizen of diagnosejaren.

Vervolgens is in Hoofdstuk 3 het verschil tussen effectiviteit en werkzaamheid (EE gap) van systemische behandelingen onderzocht in patiënten met stadium IV NSCLC, gediagnosticeerd tussen 2008 en 2014 in zes Santeon ziekenhuizen. Voor elke patiënt werd een EE factor berekend door zijn of haar totale overleving vanaf start behandeling te delen door de samengevoegde mediane overleving op basis van de klinische studies met dezelfde behandeling. Van de 2989 gediagnosticeerde patiënten begonnen 1214 patiënten (41\%) met een eerstelijnsbehandeling. Voor alle bestudeerde behandelingen met chemotherapie en doelgerichte therapie was de overleving in de dagelijkse praktijk korter dan de overleving 
zoals gerapporteerd in de klinische studies. De EE factor was 0,77 (95\% Bl 0,70-0,85; $p<0,001)$, wat betekent dat de overleving van patiënten met uitgezaaide NSCLC die in de dagelijks praktijk worden behandeld met chemotherapie of doelgerichte therapie bijna een kwart korter is dan bij de patiënten die deelnamen aan klinische studies. Dit verschil werd gedeeltelijk verklaard door de ECOG PS van de patiënten, het vroegtijdig beëindigen van de behandeling en minder volgende behandellijnen.

In Hoofdstuk 4 is over de jaren 2008-2014 de overleving van patiënten na de diagnose stadium IV NSCLC vergeleken met de ontwikkeling van de kosten voor systemische behandeling. Van de 1214 patiënten uit het onderzoek zoals beschreven in Hoofdstuk 3 zijn gegevens verzameld uit de Santeon Farmadatabase (SFD) over de cumulatieve dosering (in mg) van elk geneesmiddel uit de toegepaste systemische behandelingen tot overlijden. Deze bedragen zijn vermenigvuldigd met de kosten per milligram uitgedrukt in euro's om de totale geneesmiddelkosten per behandellijn per patiënt te berekenen. Voor geneesmiddelen met parenterale toediening werden de kosten voor een bezoek aan de dagbehandeling toegevoegd. De mediane overleving en de gemiddelde totale geneesmiddelkosten vertoonden geen significante variatie over de jaren heen. De gemiddelde totale geneesmiddelkosten per 1 jaar overleving varieerden van $€ 20.665$ tot $€ 26.438$ in de onderzochte periode. De kosten voor eerstelijns systemische behandeling waren in de jaren 2011-2014 significant hoger dan in de periode 2008-2010.

Vanwege recente ontwikkelingen in de behandeling van patiënten met gemetastaseerd NSCLC is in Hoofdstuk 5 de EE gap voor immunotherapie onderzocht bij patiënten gediagnosticeerd in de jaren 2015-2018. Progressievrije overleving en totale overleving van eerstelijnsbehandeling met pembrolizumab en tweedelijnsbehandeling met nivolumab werden vergeleken met gegevens uit klinische studies door het berekenen van hazard ratio's (HR's). Van de 1950 gediagnosticeerde patiënten begonnen 1005 (52\%) met een eerstelijnsbehandeling, van wie 83 pembrolizumab kregen. Nivolumab werd gestart als tweedelijnsbehandeling bij 141 patiënten. Voor beide behandelingen was de progressievrije overleving vergelijkbaar tussen de dagelijkse praktijk en de klinische studies (pembrolizumab: HR 1,08 (95\% BI 0,75-1,55), en nivolumab: HR 0,91 (95\% BI 0,74-1,14)). De totale overleving was significant korter in de dagelijkse praktijk voor eerstelijns pembrolizumab (mediane overleving 15,8 vs. 30,0 maanden; HR 1,55 (95\% BI 1,07-2,25)). Patiënten in de dagelijkse praktijk ontvingen minder vaak een vervolgbehandeling in vergelijking met patiënten in de klinische studies. 


\section{Uitkomsten in de dagelijkse praktijk van patiënten met uitgezaaide kleincellige longkanker (ED SCLC)}

In Hoofdstuk 6 worden gegevens over behandellijnen en overleving van een patiëntenpopulatie met extensive disease (ED) SCLC gepresenteerd. Van de 792 gediagnosticeerde patiënten met ED SCLC tussen 2008 en 2014 in zes Santeon-ziekenhuizen, begonnen 568 patiënten (72\%) met een eerstelijnsbehandeling. Van deze patiënten kreeg $41 \%$ een tweedelijnsbehandeling. Slechts 68 patiënten kregen een derdelijnsbehandeling. De gemiddelde leeftijd van alle behandelde patiënten was 66 jaar. De meerderheid van de patiënten (72\%) had bij diagnose een ECOG PS van 0 of 1. De mediane overleving van de behandelde patiënten was 7,4 maanden.

Vervolgens zijn in Hoofdstuk 7 de resultaten van het hiervoor beschreven onderzoek gebruikt om de EE gap van systemische behandelingen voor ED SCLC te onderzoeken. De mediane EE factor was 0,79 ( $p<0,001$ bij vergelijking met waarde 1), wat betekent dat de mediane overleving van patiënten met ED SCLC met een systemische behandeling in de dagelijkse praktijk 21\% korter is dan voor patiënten die deelnamen aan klinische studies. Een slechte ECOG PS ( $\geq 2$ ) en een hogere leeftijd bij diagnose (leeftijd $\geq 65$ jaar) waren onafhankelijke voorspellers voor een lage EE factor. De gemiddelde leeftijd en het percentage patiënten met ECOG $\geq 2$ in de dagelijkse praktijk waren significant verschillend van die in klinische studies (gemiddelde leeftijd 66 vs. 62 jaar, ECOG $\geq 2$ 25\% vs. 17\%; beide $p<0,001$ ).

\section{Communicatie van uitkomsten uit de dagelijkse praktijk naar patiënten}

Hoofdstuk 8 beschrijft de ontwikkeling en evaluatie van een web-based tool voor patiënten met stadium IV NSCLC en hun thoracale oncologen (TO's), gebaseerd op gegevens van 2989 patiënten met stadium IV NSCLC (uit het onderzoek in Hoofdstuk 3) in een participatief ontwerpproces. Deze web-based tool toont van verschillende systemische behandelingen zowel de resultaten uit de dagelijkse praktijk als de resultaten van klinische studies na het selecteren van patiëntkenmerken ('patients like me'), ter ondersteuning van geïnformeerde klinische besluitvorming. De tool werd geïmplementeerd en gebruikt door TO's in drie van de zes Santeon-ziekenhuizen (adoptiegraad van 50\%) en effectief gebruikt bij 48 patiënten (gebruikspercentage 29\%) van wie 17 ook deelnamen aan een telefonische enquête. Tien TO's reageerden op een onderzoek specifieke vragenlijst. Over het algemeen gaven de meeste van deze patiënten en TO's aan dat de tool voor hen toegevoegde waarde had in de klinische praktijk (respectievelijk 71\% en 83\%).

In de Algemene Discussie is het gebruik van uitkomstinformatie vanuit de dagelijkse praktijk in het algemeen en onze bevindingen ten aanzien van een bestaande EE gap in het bijzonder besproken in het bredere perspectief van de levenscyclus van geneesmiddelen. Daarnaast zijn mogelijke aanknopingspunten voor toekomstig onderzoek gegeven. 



\section{DANKWOORD}

Graag wil ik iedereen bedanken die direct of indirect heeft geholpen bij het uitvoeren van de onderzoeken en de totstandkoming van mijn proefschrift. Een paar mensen wil ik graag persoonlijk noemen.

Allereerst mijn (co)promotores; door mij ook wel 'de mannen' genoemd. Gaandeweg het project kreeg het promotieteam meer vorm. Ik heb het gewaardeerd dat jullie vanuit je eigen vakgebied en onderzoeksthema's konden bijdragen aan dit proefschrift.

Ewoudt, op jou kon ik altijd rekenen. Ik heb veel van je geleerd wat betreft het schrijven van artikelen. Fijn dat je mij de vrijheid gaf om alles rondom de data zelf te regelen. Soms had ik genoeg van je telefoontjes (op maandagochtend), maar het is overduidelijk: als jij er niet was geweest dan was ik nog lang niet klaar met dit proefschrift. Het was tegelijkertijd prettig en frustrerend dat je zo snel reageerde op mijn stukken, want dan kon én moest ik er weer wat mee. Waar ik liever 's avonds nog even door ging, was jij 's ochtends vroeg weer paraat en dat is erg efficiënt gebleken. Bedankt voor je vertrouwen en geduld tijdens dit proces! En de smaakvolle koffie die ik vaak goed kon gebruiken.

Franz, bedankt voor de prettige samenwerking en je inzichten in het klinische werk. Jij hebt hart voor patiënten én onderzoek en dat heb ik zeer gewaardeerd.

Harry, met jouw jarenlange ervaring in zowel het klinische werk als het onderzoek kon je altijd goede tips geven voor literatuurverwijzingen en punten voor de discussie. Dank daarvoor!

Olaf, dank voor je expertise op zoveel vlakken: farmacologie, epidemiologie en methodologie. Dank voor je bijdrage aan de verschillende vraagstukken, met heldere en weloverwogen uitleg.

Prof. Ton de Boer, Prof. Toine Egberts, Prof. Michel van den Heuvel, Prof. Anne-Marie Dingemans en Prof. Dirk Ubbink, hartelijk dank voor jullie tijd en moeite om mijn manuscript te beoordelen.

Bas Peters, dank voor het voorbereidende werk en je positief kritische blik op het onderzoek en de artikelen. Fijn dat je altijd bereikbaar en bereid was om mee te denken en te ondersteunen. Dank ook aan mijn stagiaires Arvid van Leeuwen en Merel Tonn; jullie hebben mij een hoop werk uit handen genomen en ik heb er zelf ook veel van geleerd. Ook de andere co-auteurs, Marjon Verschueren, Lotte van Loenhout, Lea Dijksman, Nelly van Uden, en Maarten Deenen: veel dank voor jullie bijdrage aan de artikelen in dit proefschrift.

Wim van den Bosch, Pauline Gantvoort en Leonique Niessen, dank voor de samenwerking bij Santeon in het project 'Zorg voor Uitkomst'. Dit is de basis geweest van mijn 
onderzoeken en dit proefschrift. Het laaghangende fruit van Wim bleek helaas niet altijd zo laag te hangen als gedacht, maar we hebben mooie resultaten laten zien.

In het bijzonder mijn hartelijke dank voor alle patiënten die hebben bijgedragen aan Hoofdstuk 8 van dit proefschrift. De interviews en telefonische vragenlijsten hebben zowel nuttige gegevens voor het onderzoek als waardevolle gesprekken voor mij persoonlijk opgeleverd. Bram Plouvier, op deze plek wil ik jou ook graag bedanken voor het ontwikkelen en mede vormgeven van de tool en voor de gesprekken tijdens onze carpool-ritjes naar Nijmegen. Lisanne Kastelijn, jij ook hartelijk dank voor je enthousiasme en het gebruik van de tool, wat vereeuwigd is met de razendsnelle fotoshoot!

Daarnaast ook mijn dank aan alle longartsen, ziekenhuisapothekers, data-analisten, BI-specialisten, verpleegkundig specialisten en medewerkers van de wetenschapsbureaus van de Santeon ziekenhuizen. Zonder jullie inzet had ik een stuk minder voor elkaar gekregen.

Alle collega's van het Santeon programmabureau: veel dank voor de samenwerking, gezelligheid, interesse in mijn onderzoek en dat ik altijd welkom was om aan te schuiven op kantoor. In het bijzonder mijn hartelijke dank aan jou, Pieter, voor je enthousiasme over mijn werk, het delen van inzichten over en weer en je vasthoudendheid in het kenbaar maken van alle Santeon-activiteiten, waaronder mijn project.

Promovenski van het St. Antonius Ziekenhuis, dank voor het samen werken, de kopjes koffie, gezelligheid en gesprekken. Het was fijn om zo nu en dan successen en ergernissen te kunnen delen met gelijkgestemden in het promotie-schuitje.

Oud-collega's van het Leerhuis en unit KVV van het Canisius-Wilhelmina Ziekenhuis, hartelijk dank voor de prettige werkplekken die ik bij jullie mocht hebben. De gesprekken tijdens de lunch en met mijn roomies waren fijn om even mijn gedachten te verzetten. In het bijzonder dank aan jou Sanne; jij werd naast collega ook een goede vriendin met wie ik veel deel op zowel werk als privé gebied. Koffie, sushi en skiën gingen gepaard met een lach en een traan. Dank voor de goede gesprekken, je luisterend oor en weloverwogen adviezen. Ook in tijden van zorgen en onzekerheid voor jullie stond je altijd voor mij klaar. Nieuwe collega's van het Nivel; het proefschrift was dan wel ingeleverd en goedgekeurd, mijn hoofd zat vaak nog vol met promoveren. Dank voor het begrip en de bemoedigende woorden.

Lieve (schoon)familie, vrienden, PH-buren en 'schoolpleinouders', teveel om persoonlijk op te noemen: jullie boden steun, een luisterend oor en de nodige afleiding. Veel dank voor jullie opbeurende woorden als ik er weer eens 'klaar mee was', interesse in wat ik nou eigenlijk aan het doen was en waardering voor hoe ik het allemaal weer voor elkaar kreeg. Het kwam altijd weer goed, mede dankzij de fijne gesprekken, spelletjes, koffiemomenten, escape-rooms, uitstapjes (van Keukenhof tot Glasgow), sushi-dates, Zumba-activiteiten en 
wandelingen. En natuurlijk dankzij de oppaskwaliteiten van het gezin Den Hertog.

Anne-Renske, jou wil ik graag nog even apart noemen. Wij kennen elkaar al ruim 23 jaar en hebben veel lief en leed gedeeld. Dank je wel voor de fijne gesprekken waarbij de tijd áltijd te snel voorbij is, de gezellige uitstapjes met de kinderen erbij, relaxen in de sauna en je luisterend oor.

Mijn paranimfen; jullie beiden aan mijn zijde tijdens de verdediging waardeer ik zeer. Marieke, onze vriendschap gaat al lang terug, en ondanks soms een grote fysieke afstand tussen ons, ben je altijd slechts een appje of belletje verwijderd geweest. Nooit te druk om goede raad te geven of om op jouw manier de boel weer wat lucht te geven. Hetty, ook jij bent fysiek een eind weg geweest, om daarna mijn collega te worden bij Santeon. Wat was het fijn om zowel werk als privé zaken met je te kunnen bespreken, het liefst bij de sushi, maar telefonisch is er ook zo een uur voorbij.

Lieve Hanno en Dorien, van wijze woorden tot poepluiers, alles is de afgelopen jaren voorbij gekomen. Veel dank voor jullie zorgen voor onze dochters en ons zelf, zodat we konden werken of (nog veel liever) samen skiën.

Lieve pa en ma, veel dank voor de mogelijkheden die jullie mij hebben geboden om te kunnen studeren. Dank voor de interesse in zowel het onderzoek als de gezondheidszorg die ik van jullie heb meegekregen. Pa, dank voor het goede voorbeeld geven wat betreft promoveren en voor het kritisch meelezen van de proefdruk. Ma, dank voor de liefdevolle zorgen voor onze dochters (zodat wij weer eens konden skiën, iets wat we ook aan jullie te danken hebben!) en de gezamenlijke kopjes koffie; die voorliefde heb ik niet van een vreemde!

Lieve Anna en Sophie, ik heb mij wel eens schuldig gevoeld dat ik wéér achter de laptop zat in plaats van iets gezelligs met jullie te doen. Gelukkig lagen jullie daar niet wakker van. Ik geniet met jullie mee bij elke stap die jullie in de wereld zetten. Jullie geven mij elke dag zoveel vrolijkheid en liefde, dank jullie wel!

Lieve Bram, 'mijn' Bram, ik ben dankbaar en gelukkig dat ik het leven met jou mag delen. Jouw luisterend oor, relativering, oneindige (woord)grapjes, zorgzaamheid en (nerdachtige) hulp hebben ervoor gezorgd dat ik dit proefschrift kon afronden. Het was soms veel tegelijk en door al die activiteiten in onze agenda wilde je wel eens een dikke streep zetten. Dank dat je mij op de juiste momenten motiveerde, afremde, of de ruimte gaf. Jij bent de liefste! 



\section{CURRICULUM VITAE}

Christine van der Welle werd geboren op 24 mei 1985 te Apeldoorn. In 2003 behaalde zij haar VWO diploma en ging Biomedische Wetenschappen studeren aan de Radboud Universiteit te Nijmegen. Voor haar Master koos ze voor de richting Health Technology Assessment met het Consultancy profiel, aangevuld met een minor Wetenschapscommunicatie.

$\mathrm{Na}$ het behalen van haar Master of Science diploma in 2009 startte zij als medewerker bij het Wetenschapsbureau van het Canisius-Wilhelmina Ziekenhuis (CWZ) in Nijmegen. Het begeleiden van onderzoekers, de diverse werkgroepen en het regelwerk lagen haar goed, maar zelf onderzoek doen werd gemist. Na een uitstapje als onderzoeker bij de afdeling Klinische Farmacie van het CWZ ging ze in 2014 aan de slag voor de Santeon ziekenhuizen in het project 'Zorg voor Uitkomst'. Eerst als inhoudelijk projectleider, later als data-manager en tenslotte als data-analist van patiënt gerapporteerde uitkomsten (PROMs), wat haar de titel 'PROMs-koningin' opleverde.

Het Santeon project Zorg voor Uitkomst vormde de basis voor een subsidieaanvraag bij KWF. In 2016 was het zover, de subsidie werd verleend aan deze groep niet-academische ziekenhuizen en Christine ging aan de slag als promovendus. Na het afronden van haar proefschrift is zij als postdoc onderzoeker bij het Nivel te Utrecht gestart op het themagebied 'Zorgdata en het Lerend Zorgsysteem'.

Christine is getrouwd met Bram Cramer en samen hebben zij twee dochters. 



\section{LIST OF PUBLICATIONS}

\section{Manuscripts included in this thesis}

Christine M. Cramer-van der Welle, Lotte van Loenhout, Ben E.E.M. van den Borne, Franz M.N.H. Schramel, Lea M. Dijksman. 'Care for Outcomes': systematic development of a set of outcome indicators to improve patient-relevant outcomes for patients with lung cancer. BMJ Open. 2021 Jan 15;11(1):e043229.

Christine M. Cramer - van der Welle, Franz M.N.H. Schramel, Bas J.M. Peters, John W.G. van Putten, Olaf H. Klungel, Harry J.M. Groen, Ewoudt M.W. van de Garde, for the Santeon SCLC Study Group. Systematic evaluation of the efficacy-effectiveness gap of systemic treatments in extensive disease small cell lung cancer. Pharmacoepidemiol Drug Saf. 2020 Dec 9.

Christine M. Cramer-van der Welle, Bas J.M. Peters, Maarten J. Deenen, Franz M.N.H. Schramel, Ewoudt M.W. van de Garde. Trends in Drug Costs and Overall Survival in Patients with Metastatic Non-small Cell Lung Cancer in The Netherlands Diagnosed from 2008 Through 2014. Pharmacoecon Open. 2021 Mar;5(1):121-127.

Christine M. Cramer-van der Welle, Franz M.N.H. Schramel, Arvid S. van Leeuwen, Harry J.M. Groen, Ewoudt M.W. van de Garde, for the Santeon SCLC Study Group. Real-world treatment patterns and outcomes of patients with extensive disease small cell lung cancer. Eur J Cancer Care (Engl.) 2020 Sep;29(5):e13250.

Christine M. Cramer-van der Welle, Bas J.M. Peters, Franz M.N.H. Schramel, Olaf H. Klungel, Harry J.M. Groen, Ewoudt M.W. van de Garde, for the Santeon NSCLC Study Group. Systematic evaluation of the efficacy-effectiveness gap of systemic treatments in metastatic non-small cell lung cancer. Eur Respir J. 2018 Dec 20;52(6):1801100.

Bas J.M. Peters, Christine M. Cramer-vd Welle, Arthur A.J. Smit, Franz M.N.H. Schramel, Ewoudt M.W. van de Garde, for the Santeon NSCLC Study Group. Trends in prescribing systemic treatment and overall survival for non-small cell lung cancer stage IIIB/IV in the Netherlands: 2008-2012. Cancer Epidemiol. 2017 Dec;51:1-6.

\section{Other manuscripts}

Huiskes VJB, Cramer-van der Welle CM, van den Ende CHM, van Dijk L, Bos JM, Kramers C, van den Bemt BJF. Communication about Drug-Related Problems (DRPs) during Patients' Visits to Dutch Physicians and Pharmacies. Health Commun. 2018 Dec 10:1-8.

Van Hunsel F, van der Welle C, Passier A, van Puijenbroek E, van Grootheest K. Motives for reporting adverse drug reactions by patient-reporters in the Netherlands. Eur J Clin Pharmacol. 2010 Nov;66(11):1143-50. 


